\title{
Pathwise Uniqueness for Stochastic Heat Equations with Hölder Continuous Coefficients: the White Noise Case
}

\author{
Leonid Mytnik $^{1} \quad$ Edwin Perkins ${ }^{2}$ \\ Faculty of Industrial Engineering and Management, \\ Technion - Israel Institute of Technology, Haifa 32000, Israel \\ E-mail address: leonid@ie.technion.ac.il \\ Department of Mathematics, The University of British Columbia, \\ 1984 Mathematics Road, Vancouver, B.C., Canada V6T 1Z2 \\ E-mail address: perkins@math.ubc.ca
}

\begin{abstract}
We prove pathwise uniqueness for solutions of parabolic stochastic pde's with multiplicative white noise if the coefficient is Hölder continuous of index $\gamma>3 / 4$. The method of proof is an infinite-dimensional version of the Yamada-Watanabe argument for ordinary stochastic differential equations.
\end{abstract}

November 13, 2018

AMS 2000 subject classifications. Primary 60H15. Secondary 60G60, 60H10, 60H40, 60K35, 60J80.

Keywords and phrases. Stochastic partial differential equations, pathwise uniqueness, white noise.

Running head. Pathwise uniqueness for SPDE's

1. Supported in part by the Israel Science Foundation (grant No. 1162/06).

2. Supported by an NSERC Research grant. 


\section{Introduction}

Let $\sigma: \mathbb{R}_{+} \times \mathbb{R}^{2} \rightarrow \mathbb{R}$ and consider the stochastic heat equation

$$
\frac{\partial}{\partial t} X(t, x)=\frac{1}{2} \Delta X(t, x) d t+\sigma(t, x, X(t, x)) \dot{W}(x, t)+b(t, x, X(t, x)) .
$$

Here $\Delta$ denotes the Laplacian and $\dot{W}$ is space-time white noise on $\mathbb{R}_{+} \times \mathbb{R}$. If $\sigma(t, x, X)$ and $b(t, x, X)$ are Lipschitz continuous in $X$ it is well-known that there are pathwise unique solutions to (1.1) (see (Wal86) $)$. When $\sigma(t, x, X)=\sqrt{f(t, x, X) X}$ such equations arise naturally as the scaling limits of critical branching particle systems where the branching rate at $(t, x)$ is given by $f(t, x, X(t, x))$ and $X(t, x)$ is a measure of the local particle density at $(t, x)$. Such coefficients are not Lipschitz continuous and pathwise uniqueness remains open even in the case where $f \equiv 1$, $b \equiv 0$ and $X$ is the density of super-Brownian motion (see Section III.4 of (Per02)). In this case, and more generally for $f=X^{p}$ for $p>0$, uniqueness in law is known by duality arguments (see (Myt99)). The duality arguments are highly non-robust, however, and pathwise uniqueness, if true, would typically hold for a much less restrictive set of coefficients. Our goal in this work is to show pathwise uniqueness holds for solutions to (1.1) if $\sigma(t, x, \cdot)$ is Hölder continuous of order $\gamma$ and $\gamma>3 / 4$. The attentive reader will have already noted that the motivating example given above does not satisfy this condition.

The above equation does have the advantage of having a diagonal form-that is, when viewed as a continuum-dimensional stochastic differential equation there are no off-diagonal terms in the noise part of the equation and the diffusion coefficient for the $x$ coordinate is a function of that coordinate alone. For finite-dimensional sde's this was the setting for Yamada and Watanabe's extension (YW71) of Itô's pathwise uniqueness results to Hölder $(1 / 2)$ continuous coefficients, and so our plan will be to carry over their approach to our infinite dimensional setting. This programme was already carried out in the context of coloured noise in (MPS06), but the methods used there when specialized to white noise given nothing beyond the classical Lipschitz uniqueness. In fact for coloured noise in higher dimensions the results in (MPS06) did not even come close to the known results on pathwise uniqueness for Lipschitz continuous coefficients (Dal99)-see the discussion after Remark 1.5 in (MPS06). This is what led to our belief that there was room for substantial improvement in the methods of (MPS06) and hence to the present work.

We introduce a growth condition, a Hölder continuity condition on $\sigma$ and the standard Lipschitz condition on $b$ :

for some $\gamma>3 / 4$ there are $R_{1}, R_{2}>0$ and for all $T>0$ there is an $R_{0}(T)$

so that for all $t \in[0, T]$ and all $\left(x, X, X^{\prime}\right) \in \mathbb{R}^{3}$,

$$
\left|\sigma(t, x, X)-\sigma\left(t, x, X^{\prime}\right)\right| \leq R_{0}(T) e^{R_{1}|x|}\left(1+|X|+\left|X^{\prime}\right|\right)^{R_{2}}\left|X-X^{\prime}\right|^{\gamma}
$$

and

(1.4) there is a $B>0$ s.t. for all $\left(t, x, X, X^{\prime}\right) \in \mathbb{R}_{+} \times \mathbb{R}^{3},\left|b(t, x, X)-b\left(t, x, X^{\prime}\right)\right| \leq B\left|X-X^{\prime}\right|$. 
We assume $W$ is a white noise on the filtered probability space $\left(\Omega, \mathcal{F}, \mathcal{F}_{t}, \mathbb{P}\right)$, where $\mathcal{F}_{t}$ satisfies the usual hypotheses. This means $W_{t}(\phi)$ is an $\mathcal{F}_{t}$-Brownian motion with variance $\|\phi\|_{2}^{2}$ for each $\phi \in \mathbb{L}^{2}(\mathbb{R}, d x)$ and $W_{t}(\phi)$ and $W_{t}(\psi)$ are independent if $\int \phi(x) \psi(x) d x=0$. We set $p_{t}(x)=$ $(2 \pi t)^{-1 / 2} \exp \left\{-x^{2} / 2 t\right\}, P_{t} f(x)=\int f(y) p_{t}(y-x) d y$, and let $\mathcal{F}_{t}^{W} \subset \mathcal{F}_{t}$ be the filtration generated by $W$ satisfying the usual hypotheses. A stochastic process $X: \Omega \times \mathbb{R}_{+} \times \mathbb{R} \rightarrow \mathbb{R}$, which is jointly measurable and $\mathcal{F}_{t}$-adapted, is said to be a solution to the stochastic heat equation (1.1) on $\left(\Omega, \mathcal{F}, \mathcal{F}_{t}, \mathbb{P}\right)$ with initial condition $X_{0}: \mathbb{R} \rightarrow \mathbb{R}$, if for each $t \geq 0$, and $x \in \mathbb{R}$,

$$
\begin{aligned}
X(t, x)=\int_{\mathbb{R}} p_{t}(y-x) X_{0}(y) d y & +\int_{0}^{t} \int_{\mathbb{R}} p_{t-s}(y-x) \sigma(s, y, X(s, y)) W(d s, d y) \\
& +\int_{0}^{t} \int_{\mathbb{R}} p_{t-s}(y-x) b(s, y, X(s, y)) d y d s \text { a.s. }
\end{aligned}
$$

To state the main results we introduce some notation, which will be used throughout this work: If $E \subset \mathbb{R}^{d}$, we write $C(E)$ for the space of continuous functions on $E$. A superscript $k$, respectively $\infty$, indicates that functions are in addition $k$ times, respectively infinitely often, continuously differentiable. A subscript $b$, respectively $c$, indicates that they are also bounded, respectively have compact support. We also define

$$
\|f\|_{\lambda}:=\sup _{x \in \mathbb{R}}|f(x)| e^{-\lambda|x|},
$$

set $C_{\text {tem }}:=\left\{f \in C(\mathbb{R}),\|f\|_{\lambda}<\infty\right.$ for any $\left.\lambda>0\right\}$ and endow it with the topology induced by the norms $\|\cdot\|_{\lambda}$ for $\lambda>0$. That is, $f_{n} \rightarrow f$ in $C_{\text {tem }}$ iff $d\left(f, f_{n}\right)=\sum_{k=1}^{\infty} 2^{-k}\left(\left\|f-f_{n}\right\|_{1 / k} \wedge 1\right) \rightarrow 0$ as $n \rightarrow \infty$. Then $\left(C_{t e m}, d\right)$ is a Polish space. By identifying the white noise $W$, with the associated Brownian sheet, we may view $W$ as a stochastic processes with sample paths in $C\left(\mathbb{R}_{+}, C_{t e m}\right)$. Here as usual, $C\left(\mathbb{R}_{+}, C_{t e m}\right)$ is given the topology of uniform convergence on compacts.

A stochastically weak solution to (1.1) is a solution on some filtered space with respect to some noise $W$, i.e., the noise and space are not specified in advance.

With this notation we can state the following standard existence result whose proof is a minor modification of Theorem 1.2 of (MPS06) and is given in the next Section.

Theorem 1.1 Let $X_{0} \in C_{\text {tem }}$, and let $b, \sigma: \mathbb{R}_{+} \times \mathbb{R}^{2} \rightarrow \mathbb{R}$ satisfy (1.2), (1.3), and (1.4). Then there exists a stochastically weak solution to (1.1) with sample paths a.s. in $C\left(\mathbb{R}_{+}, C_{\text {tem }}\right)$.

We say pathwise uniqueness holds for solutions of (1.1) in $C\left(\mathbb{R}_{+}, C_{t e m}\right)$ if for every $X_{0} \in C_{t e m}$, any two solutions to (1.1) with sample paths a.s. in $C\left(\mathbb{R}_{+}, C_{t e m}\right)$ must be equal with probability 1. For Lipschitz continuous $\sigma$, this follows from Theorem 2.2 of (Shi94). Here then is our main result:

Theorem 1.2 Assume that $b, \sigma: \mathbb{R}_{+} \times \mathbb{R}^{2} \rightarrow \mathbb{R}$ satisfy (1.2), (1.3) and (1.4). Then pathwise uniqueness holds for solutions of (1.1) in $C\left(\mathbb{R}_{+}, C_{\text {tem }}\right)$.

As an immediate consequence of Theorems 1.1 and 1.2 we get existence and uniqueness of strong solutions and joint uniqueness in law of $(X, W)$.

Theorem 1.3 Assume that $b, \sigma: \mathbb{R}_{+} \times \mathbb{R}^{2} \rightarrow \mathbb{R}$ satisfy (1.2), (1.3) and (1.4). Then for any $X_{0} \in$ $C_{\text {tem }}$ there is a solution $X$ to (1.1) on $\left(\Omega, \mathcal{F}_{\infty}^{W}, \mathcal{F}_{t}^{W}, \mathbb{P}\right)$ with sample paths a.s. in $C\left(\mathbb{R}_{+}, C_{\text {tem }}\right)$. If $X^{\prime}$ is any other solution to (1.1) on $\left(\Omega, \mathcal{F}, \mathcal{F}_{t}, \mathbb{P}\right)$ with sample paths a.s. in $C\left(\mathbb{R}_{+}, C_{\text {tem }}\right)$, then $X(t, x)=$ $X^{\prime}(t, x)$ for all $t, x$ a.s. The joint law $P_{X_{0}}$ of $(X, W)$ on $C\left(\mathbb{R}_{+}, C_{\text {tem }}\right)$ is uniquely determined by $X_{0}$ and is Borel measurable in $X_{0}$. 
Proof. The Borel measurability of the law is proved as in Exercise 6.7.4 in (SV79). We now apply Theorem 3.14 of (Kur07), with the Polish state spaces $S_{1}$ and $S_{2}$ for the driving process $(W)$ and solution $(X)$ in that work both equal to $C\left(\mathbb{R}_{+}, C_{\text {tem }}\right)$. Theorems 1.1 and 1.2 imply the hypotheses of weak existence and pointwise uniqueness of (a) of that result. The conclusions of an $\mathcal{F}_{t}^{W}$-adapted (strong) solution and uniqueness in law of $(X, W)$ follow from the conclusions in Theorem 3.14 (b) (of (Kur07)) of a strong compatible solution and joint uniqueness in law, respectively. (Note that Lemma 3.11 of the above reference shows that a strong, compatible solution must be $\mathcal{F}_{t}^{W}$-adapted.)

Remark 1.4 (a) When assuming (1.2), it suffices to assume (1.3) for $\left|X-X^{\prime}\right| \leq 1$. Indeed this condition is immediate from (1.2) for $\left|X-X^{\prime}\right| \geq 1$ with $R_{1}=0$ and $R_{2}=1$.

(b) (1.3) implies the local Hölder condition:

$$
\begin{gathered}
\text { for some } \gamma>3 / 4 \text { for all } K>0 \text { there is an } L_{K} \text { so that for all } t \in[0, K] \\
\text { and } x, X_{1}, X_{2} \in[-K, K],\left|\sigma\left(t, x, X_{1}\right)-\sigma\left(t, x, X_{2}\right)\right| \leq L_{K}\left|X_{1}-X_{2}\right|^{\gamma} \text {. }
\end{gathered}
$$

In fact it prescribes the growth rate of the Hölder constants $L_{K}$ (polynomial in $X$ and exponential in $x)$.

In order to give a bit of intuition for Theorem 1.2, we recall the result from (MPS06) which dealt with the stochastic heat equation driven by coloured noise. Let $\dot{W}(t, x)$ be the mean zero Gaussian noise on $\mathbb{R}_{+} \times \mathbb{R}^{d}$ with covariance given by

$$
E[\dot{W}(t, x) \dot{W}(s, y)]=\delta_{0}(t-s) k(x-y)
$$

where

$$
k(x-y) \leq c|x-y|^{-\alpha}
$$

for some $\alpha \in(0, d \wedge 2)$. Note that the white noise considered in this paper is the case $k(x)=\delta_{0}(x)$. It formally corresponds to $\alpha=1$ in dimension $d=1$. Now let $X$ satisfies the SPDE:

$$
\frac{\partial}{\partial t} X(t, x)=\frac{1}{2} \Delta X(t, x) d t+\sigma(X(t, x)) \dot{W}(x, t),
$$

with $\dot{W}$ being the coloured noise just described. Then the following result was proved in (MPS06).

Theorem 1.5 ((MPS06)) For $\alpha<2 \gamma-1$, pathwise uniqueness holds for (1.9)).

Let $\tilde{u}=X^{1}-X^{2}$ be the difference of two solutions to (1.9). The the proof of Theorem 1.5 relied on a study of the Hölder continuity of $\tilde{u}(t, \cdot)$ at points where $\tilde{u}(t, x)$ is "small". Let $\xi$ be the Hölder exponent of $\tilde{u}(t, \cdot)$ at such points. The following connection between parameter $\xi$ and the pathwise uniqueness was shown in (MPS06) (see condition (41) in the proof of Theorem 4.1 there): If

$$
\alpha<\xi(2 \gamma-1)
$$


then pathwise uniqueness holds for (1.9). Hence, the better the regularity one has for $\tilde{u}$ near its zero set, the "weaker" the hypotheses required for pathwise uniqueness. It was shown in (MPS06) that at the points $x$ where $\tilde{u}(t, x)$ is "small", $\tilde{u}(t, \cdot)$ is Hölder continuous with any exponent $\xi$ such that

$$
\xi<\frac{1-\frac{\alpha}{2}}{1-\gamma} \wedge 1
$$

(For the precise statement of this result see Theorem 2.2 in the next section.) Note that in the case when $\alpha<2 \gamma-1$, (1.11) turns into the following condition

$$
\xi<1,
$$

and this together with (1.10) imply Theorem 1.5.

Now assume $\dot{W}$ is white noise on $\mathbb{R}_{+} \times \mathbb{R}$ and $d=1$. This formally corresponds to the $\alpha=1$, and in this case the conditions (1.10), (1.11) can be written as

$$
\begin{aligned}
& 1<\xi(2 \gamma-1) \\
& \xi<\frac{1}{2(1-\gamma)} \wedge 1 .
\end{aligned}
$$

For $\gamma \geq 1 / 2$, we have $\frac{1}{2(1-\gamma)} \geq 1$ and hence one can take $\xi<1$ arbitrarily close to 1 (a proof of this is given in Theorem 2.3 below), substitute it into (1.13), and get a vacuous condition for pathwise uniqueness, namely

$$
\gamma>1
$$

To improve on this we will need to get more refined information on the difference, $u$, of two solutions to (1.1) near the points $x_{0}$ where $u\left(t, x_{0}\right) \approx 0$. To be more precise, suppose one is able to show that

$$
|u(t, x)| \leq c\left|x-x_{0}\right|^{\xi}
$$

for any

$$
\xi<\frac{1}{2(1-\gamma)} \wedge 2
$$

By substituting the upper bound for $\xi$ from (1.16) into (1.13) and doing a bit of arithmetic one gets the following condition for pathwise uniqueness

$$
\gamma>3 / 4
$$

which is the result claimed in Theorem 1.2. We will in fact verify a version of (1.15) under (1.16) and $\gamma>3 / 4$. A more detailed description of our approach, is given in Section 2 ,

The above discussion allows us to conjecture a stronger result on pathwise uniqueness for the case of equations driven by a coloured noise:

Conjecture 1.6 If

$$
\alpha<2(2 \gamma-1)
$$

then pathwise uniqueness holds for (1.9). 
The reasoning for this conjecture is similar to that for the white noise case. Let $\tilde{u}$ again be the difference of two solutions to (1.9). Suppose that if $\tilde{u}\left(t, x_{0}\right) \approx 0$ then at the points nearby we have

$$
\tilde{u}(t, x) \leq c\left|x-x_{0}\right|^{\xi}
$$

for any

$$
\xi<\frac{1-\frac{\alpha}{2}}{1-\gamma} \wedge 2
$$

By substituting the upper bound for $\xi$ from (1.20) into (1.10) and simple algebra one gets (1.18) as a condition for pathwise uniqueness for (1.9). Note that (1.18) can be equivalently written as

$$
\gamma>\frac{1}{2}+\frac{\alpha}{4}
$$

In the next Section we give a quick proof of Theorem 1.1 and then turn to the main result, Theorem 1.2. Following the natural analogue of the Yamada-Watanabe argument for stochastic pde's, as in (MPS06), the problem quickly reduces to one of showing that the analogue of the local time term is zero (Proposition 2.1). As described above, the key ingredient here will be tight control on the spatial behaviour of the difference of two solutions, when this difference is very small, that is, when the solutions separate. Roughly speaking, as in Yamada and Watanabe's argument we first show that solutions must separate in a gentlemanly manner and therefore cannot separate at all. Section 2 includes a heuristic description of the method and further explanation of why $\gamma=3 / 4$ is critical in our approach. It also gives an outline of the contents of the entire paper.

Convention on Constants. Constants whose value is unimportant and may change from line to line are denoted $c_{1}, c_{2}, \ldots$, while constants whose values will be referred to later and appear initially in say, Lemma i.j are denoted $c_{i . j}$ or $C_{i . j}$.

Acknowledgements. The second author thanks the Technion for hosting him during a visit where some of this research was carried out. This project was initiated during the visit of the first author to the UBC where he participated in a Workshop on SPDE's sponsored by PIMS and thanks go to the Pacific Institute for the Mathematical Sciences for its support.

\section{Proof of Theorems 1.1 and 1.2}

Proof of Theorem 1.1 This is standard so we only give a sketch and set $b \equiv 0$ for simplicity. By taking weak limits as $T \rightarrow \infty$ we may assume $R_{0}(T)=R_{0}$ is independent of $T$. Choose a symmetric $\psi_{n} \in C_{c}^{\infty}$ so that $0 \leq \psi_{n} \leq 1,\left\|\psi_{n}^{\prime}\right\|_{\infty} \leq 1, \psi_{n}(x)=1$ if $|x| \leq n$ and $\psi_{n}(x)=0$ if $|x| \geq n+2$. Let

$$
\sigma_{n}(t, x, X)=\int \sigma\left(t, x, X^{\prime}\right) p_{2^{-n}}\left(X^{\prime}-X\right) d X^{\prime} \psi_{n}(X)
$$

It is easy to then check the following:

$$
\left|\sigma_{n}(t, x, X)\right| \leq 2 q_{1.2}(1+|X|),
$$




$$
\left|\sigma_{n}\left(t, x, X^{\prime}\right)-\sigma_{n}(t, x, X)\right| \leq c_{n}\left|X^{\prime}-X\right|
$$

and

$$
\begin{aligned}
\left|\sigma_{n}(t, x, X)-\sigma(t, x, X)\right| & \leq \underset{2.24}{ }\left[e^{R_{1}|x|}\left(|X|^{R_{2}}+1\right) 2^{-n \gamma / 2}+(1+|X|)\left(1-\psi_{n}(|X|)\right]\right. \\
& \rightarrow 0 \text { uniformly on compacts as } n \rightarrow \infty .
\end{aligned}
$$

Use (2.22), (2.23) and Theorem 2.2 of (Shi94) to see there are solutions $X^{n}$ to (1.5) $)_{n}$ (all with respect to $W$ )-here (1.5) $n_{n}$ is (1.5) but with $\sigma_{n}$ in place of $\sigma$. Now argue as in Section 6 of (Shi94) (see the proof of Theorem 2.2) or in the derivation of Theorem 1.2 of (MPS06) (the present white noise setting simplifies those arguments) to see that $\left\{X^{n}\right\}$ is tight in $C\left(\mathbb{R}_{+}, C_{t e m}\right)$. More specifically, using the growth condition (1.2), it is straightforward to carry over the proof of Proposition 1.8(a) of (MPS06) (see Lemmas A.3 and A.5 of that paper) and show

$$
\text { for all } T, \lambda, p>0, \quad \sup _{n} E\left(\sup _{0 \leq t \leq T} \sup _{x \in \mathbb{R}}\left|X^{n}(t, x)\right|^{p} e^{-\lambda|x|}\right)<\infty .
$$

The above bounds in turn give uniform bounds on the $p$ th moments of the space-time increments of $X^{n}$ (see Lemma A.4 of (MPS06)) and hence tightness. Indeed, the orthogonality of white noise makes all these calculation somewhat easier. By Skorohod's theorem we may assume $X^{n_{k}}$ converges a.s. to $X$ in $C\left(\mathbb{R}_{+}, C_{t e m}\right)$ on some probability space. It is now easy to use (2.24) to see that (perhaps on a larger space), $X$ solves (1.5).

Next consider Theorem 1.2 and assume its hypotheses throughout. By Remark 1.4(a) decreasing $\gamma$ only weakens the hypotheses and so we may, and shall, assume that

$$
3 / 4<\gamma<1 \text {. }
$$

Let $X^{1}$ and $X^{2}$ be two solutions of $(1.5)$ on $\left(\Omega, \mathcal{F}, \mathcal{F}_{t}, \mathbb{P}\right)$ with sample paths in $C\left(\mathbb{R}_{+}, C_{\text {tem }}\right)$ a.s., with the same initial condition, $X^{1}(0)=X^{2}(0)=X_{0} \in C_{\text {tem }}$, and of course the same noise $W$. For adapted processes with sample paths in $C\left(\mathbb{R}_{+}, C_{t e m}\right)$, (1.5) is equivalent to the distributional form of (1.1) (see Theorem 2.1 of (Shi94)). That is, for $i=1,2$ and $\Phi \in C_{c}^{\infty}(\mathbb{R})$ :

$$
\begin{aligned}
\int_{\mathbb{R}} X^{i}(t, x) \Phi(x) d x= & \int_{\mathbb{R}} X_{0}^{i}(x) \Phi(x) d x+\int_{0}^{t} \int_{\mathbb{R}} X^{i}(s, x) \frac{1}{2} \Delta \Phi(x) d x d s \\
& +\int_{0}^{t} \int_{\mathbb{R}} \sigma\left(s, x, X^{i}(s, x)\right) \Phi(x) W(d s, d x) \\
& +\int_{0}^{t} \int_{\mathbb{R}} b\left(s, x, X^{i}(s, x)\right) \Phi(x) d x d s \quad \forall t \geq 0 \quad \text { a.s. }
\end{aligned}
$$

Let

$$
T_{K}=\inf \left\{s \geq 0: \sup _{y}\left(\left|X^{1}(s, y)\right| \vee\left|X^{2}(s, y)\right|\right) e^{-|y|}>K\right\} \wedge K .
$$

We first show that (1.3) may be strengthened to

$$
\begin{aligned}
& \text { for some } 1>\gamma>3 / 4 \text { there are } R_{0}, R_{1} \geq 1 \text { so that for all } t \geq 0 \\
& \text { and all }\left(x, X, X^{\prime}\right) \in \mathbb{R}^{3},\left|\sigma(t, x, X)-\sigma\left(t, x, X^{\prime}\right)\right| \leq R_{0} e^{R_{1}|x|}\left|X-X^{\prime}\right|^{\gamma} \text {. }
\end{aligned}
$$


Assume that Theorem 1.2 holds under (2.29) and that $\sigma$ satisfies (1.3). Define

$$
\sigma_{K}(t, x, X)=\sigma\left(t, x,\left(X \vee\left(-K e^{|x|}\right)\right) \wedge K e^{|x|}\right) 1(t \leq K) .
$$

Then

$$
\left|\sigma_{K}(t, x, X)-\sigma_{K}\left(t, x, X^{\prime}\right)\right| \leq R_{0}(K) e^{R_{1}|x|}\left(1+2 K e^{|x|}\right)^{R_{2}}\left|X-X^{\prime}\right|^{\gamma},
$$

and so (2.29) holds with $R_{1}+R_{2}$ in place of $R_{1}$ (the restriction that $R_{i} \geq 1$ is for convenience and is no restriction). Providing that for $\lambda=1,\left\|X_{0}\right\|_{\lambda}<K$, we have

$$
\sigma\left(t, x, X^{i}(t, x)\right)=\sigma_{K}\left(t, x, X^{i}(t, x)\right) \text { for all } x \text { and } t \leq T_{K} .
$$

Therefore $\sigma_{K}$ satisfies(2.29) and of course (1.2). So we may apply Theorem [1.3 with $\sigma_{K}$ in place of $\sigma$. Using the law $P_{K, X_{0}}$ of $(X, W)$ on $C\left(\mathbb{R}_{+}, C_{t e m}\right)^{2}$ (Borel in $\left.X_{0}\right)$ it is easy now to continue the solutions $X^{i}$ to $(2.27)_{K}$ (the $K$ reminds us we are dealing with $\sigma_{K}$ ) beyond $T_{K}$ and construct solutions $\tilde{X}^{i}, i=1,2$ to $(2.27)_{K}$ starting at $X_{0}$ such that $\left(\tilde{X}^{1}\left(\cdot \wedge T_{K}\right), \tilde{X}^{2}\left(\cdot \wedge T_{K}\right)\right)$ is equal in law to $\left(X^{1}\left(\cdot \wedge T_{K}\right), X^{2}\left(\cdot \wedge T_{K}\right)\right)$. By pathwise uniqueness in (2.27) $K$ we get $\tilde{X}^{1}=\tilde{X}^{2}$ and so $X^{1}\left(\cdot \wedge T_{K}\right)=X^{2}\left(\cdot \wedge T_{K}\right)$. Letting $K \rightarrow \infty$ gives $X^{1}=X^{2}$, as required.

We now follow the approach in Section 2 of (MPS06) and reduce the theorem to showing the analogue of the "local time term" in the Yamada-Watanabe proof is zero. Let

$$
a_{n}=\exp \{-n(n+1) / 2\}
$$

so that

$$
a_{n+1}=a_{n} e^{-n-1}=a_{n} a_{n}^{2 / n}
$$

Define functions $\psi_{n} \in C_{c}^{\infty}(\mathbb{R})$ such that $\operatorname{supp}\left(\psi_{n}\right) \subset\left(a_{n}, a_{n-1}\right)$, and

$$
0 \leq \psi_{n}(x) \leq \frac{2}{n x} \quad \text { for all } x \in \mathbb{R} \text { as well as } \quad \int_{a_{n}}^{a_{n-1}} \psi_{n}(x) d x=1 .
$$

Finally, set

$$
\phi_{n}(x)=\int_{0}^{|x|} \int_{0}^{y} \psi_{n}(z) d z d y
$$

From this it is easy to see that $\phi_{n}(x) \uparrow|x|$ uniformly in $x$. Note that each $\psi_{n}$, and thus also each $\phi_{n}$, is identically zero in a neighborhood of zero. This implies that $\phi_{n} \in C^{\infty}(\mathbb{R})$ despite the absolute value in its definition. We have

$$
\begin{aligned}
& \phi_{n}^{\prime}(x)=\operatorname{sgn}(x) \int_{0}^{|x|} \psi_{n}(y) d y, \\
& \phi_{n}^{\prime \prime}(x)=\psi_{n}(|x|) .
\end{aligned}
$$

Thus, $\left|\phi_{n}^{\prime}(x)\right| \leq 1$, and $\int \phi_{n}^{\prime \prime}(x) h(x) d x \rightarrow h(0)$ for any function $h$ which is continuous at zero. Define

$$
u \equiv X^{1}-X^{2}
$$


Let $\Phi \in C_{c}^{\infty}(\mathbb{R})$ satisfy $0 \leq \Phi \leq 1, \operatorname{supp}(\Phi) \subset(-1,1)$ and $\int_{\mathbb{R}} \Phi(x) d x=1$, and set $\Phi_{x}^{m}(y)=$ $m \Phi(m(x-y))$. Let $\langle\cdot, \cdot\rangle$ denote the scalar product on $\mathbb{L}^{2}(\mathbb{R})$. By applying Itô's Formula to the semimartingales $\left\langle X_{t}^{i}, \Phi_{x}^{m}\right\rangle$ in (2.27) it follows that

$$
\begin{aligned}
& \phi_{n}\left(\left\langle u_{t}, \Phi_{x}^{m}\right\rangle\right) \\
& =\int_{0}^{t} \int_{\mathbb{R}} \phi_{n}^{\prime}\left(\left\langle u_{s}, \Phi_{x}^{m}\right\rangle\right)\left(\sigma\left(s, y, X^{1}(s, y)\right)-\sigma\left(s, y, X^{2}(s, y)\right)\right) \Phi_{x}^{m}(y) W(d s, d y) \\
& \quad+\int_{0}^{t} \phi_{n}^{\prime}\left(\left\langle u_{s}, \Phi_{x}^{m}\right\rangle\right)\left\langle u_{s}, \frac{1}{2} \Delta \Phi_{x}^{m}\right\rangle d s \\
& \quad+\frac{1}{2} \int_{0}^{t} \int_{\mathbb{R}} \psi_{n}\left(\left|\left\langle u_{s}, \Phi_{x}^{m}\right\rangle\right|\right)\left(\sigma\left(s, y, X^{1}(s, y)\right)-\sigma\left(s, y, X^{2}(s, y)\right)\right)^{2} \\
& \quad \times \Phi_{x}^{m}(y)^{2} d y d s \\
& \quad+\int_{0}^{t} \int_{\mathbb{R}} \phi_{n}^{\prime}\left(\left\langle u_{s}, \Phi_{x}^{m}\right\rangle\right)\left(b\left(s, y, X^{1}(s, y)\right)-b\left(s, y, X^{2}(s, y)\right)\right) \Phi_{x}^{m}(y) d y d s .
\end{aligned}
$$

We integrate this function of $x$ against another non-negative test function $\Psi \in C_{c}^{\infty}\left(\left[0, t_{0}\right] \times \mathbb{R}\right)$ $\left(t_{0} \in(0, \infty)\right)$. Choose $K_{1} \in \mathbb{N}$ large so that for $\lambda=1$,

$$
\left\|X_{0}\right\|_{\lambda}<K_{1} \text { and } \Gamma \equiv\left\{x: \Psi_{s}(x)>0 \exists s \leq t_{0}\right\} \subset\left(-K_{1}, K_{1}\right) .
$$

We then obtain by the classical and stochastic versions of Fubini's Theorem (see Theorem 2.6 of (Wal86) for the latter), and arguing as in the proof of Proposition II.5.7 of (Per02) to handle the time dependence in $\Psi$, that for any $t \in\left[0, t_{0}\right]$,

$$
\begin{aligned}
& \left\langle\phi_{n}\left(\left\langle u_{t}, \Phi_{.}^{m}\right\rangle\right), \Psi_{t}\right\rangle \\
& =\int_{0}^{t} \int_{\mathbb{R}}\left\langle\phi_{n}^{\prime}\left(\left\langle u_{s}, \Phi_{\cdot}^{m}\right\rangle\right) \Phi_{\cdot}^{m}(y), \Psi_{s}\right\rangle\left(\sigma\left(s, y, X^{1}(s, y)\right)-\sigma\left(s, y, X^{2}(s, y)\right)\right) W(d s, d y) \\
& \quad+\int_{0}^{t}\left\langle\phi_{n}^{\prime}\left(\left\langle u_{s}, \Phi_{.}^{m}\right\rangle\right)\left\langle u_{s}, \frac{1}{2} \Delta \Phi_{.}^{m}\right\rangle, \Psi_{s}\right\rangle d s \\
& \quad+\frac{1}{2} \int_{0}^{t} \int_{\mathbb{R}^{2}} \psi_{n}\left(\mid\left\langle u_{s}, \Phi_{x}^{m}\right\rangle\right)\left(\sigma\left(s, y, X^{1}(s, y)\right)-\sigma\left(s, y, X^{2}(s, y)\right)\right)^{2} \\
& \quad \times \Phi_{x}^{m}(y)^{2} d y \Psi_{s}(x) d x d s+\int_{0}^{t}\left\langle\phi_{n}\left(\left\langle u_{s}, \Phi_{\cdot}^{m}\right\rangle\right), \dot{\Psi}_{s}\right\rangle d s \\
& +\int_{0}^{t} \int_{\mathbb{R}}\left\langle\phi_{n}^{\prime}\left(\left\langle u_{s}, \Phi_{\cdot}^{m}\right\rangle\right) \Phi_{\cdot}^{m}(y), \Psi_{s}\right\rangle\left(b\left(s, y, X^{1}(s, y)\right)-b\left(s, y, X^{2}(s, y)\right)\right) d y d s \\
& \equiv I_{1}^{m, n}(t)+I_{2}^{m, n}(t)+I_{3}^{m, n}(t)+I_{4}^{m, n}(t)+I_{5}^{m, n}(t) .
\end{aligned}
$$

The expectation condition in Walsh's Theorem 2.6 may be realized by localization, using the stopping times $\left\{T_{K}\right\}$.

Set $m_{n}=a_{n-1}^{-1 / 2}=\exp \{(n-1) n / 4\}$ for $n \in \mathbb{N}$. In the integral defining $I_{3}^{m_{n+1}, n+1}$ we may assume $|x| \leq K_{1}$ by (2.36) and so $|y| \leq K_{1}+1$. Let $K \in \mathbb{N}^{\geq K_{1}}$. If $s \leq T_{K}$, then for such a $y$,

$$
\left|X^{i}(s, y)\right| \leq K e^{|y|} \leq K e^{\left(K_{1}+1\right)} \text { for } i=1,2 .
$$


Therefore (1.6), (2.32) and (2.31) show that if $K^{\prime}=K e^{\left(K_{1}+1\right)}\left(\geq K_{1}+1\right)$, then for all $t \in\left[0, t_{0}\right]$,

$$
\begin{aligned}
& I_{3}^{m_{n+1}, n+1}\left(t \wedge T_{K}\right) \\
& \leq \frac{1}{2} \int_{0}^{t \wedge T_{K}} \iint 2(n+1)^{-1}\left|\left\langle u_{s}, \Phi_{x}^{m_{n+1}}\right\rangle\right|^{-1} 1\left(a_{n+1}<\left|\left\langle u_{s}, \Phi_{x}^{m_{n+1}}\right\rangle\right|<a_{n}\right) \\
& \times L_{K^{\prime}}^{2}|u(s, y)|^{2 \gamma} m_{n+1} \Phi_{x}^{m_{n+1}}(y) \Psi_{s}(x) d y d x d s \\
& \leq L_{K^{\prime}}^{2} a_{n+1}^{-1} a_{n}^{-1 / 2} \int_{0}^{t \wedge T_{K}} \iint 1\left(a_{n+1}<\left|\left\langle u_{s}, \Phi_{x}^{m_{n+1}}\right\rangle\right|<a_{n}\right)|u(s, y)|^{2 \gamma} \Phi_{x}^{m_{n+1}}(y) \Psi_{s}(x) d y d x d s \\
& (2.39) \leq L_{K^{\prime}}^{2} a_{n}^{-3 / 2-2 / n} \int_{0}^{t \wedge T_{K}} \iint 1\left(a_{n+1}<\left|\left\langle u_{s}, \Phi_{x}^{m_{n+1}}\right\rangle\right|<a_{n}\right)|u(s, y)|^{2 \gamma} \Phi_{x}^{m_{n+1}}(y) \Psi_{s}(x) d y d x d s .
\end{aligned}
$$

We define

$$
I^{n}(t)=a_{n}^{-3 / 2-2 / n} \int_{0}^{t} \iint 1\left(\left|\left\langle u_{s}, \Phi_{x}^{m_{n+1}}\right\rangle\right|<a_{n}\right)|u(s, y)|^{2 \gamma} \Phi_{x}^{m_{n+1}}(y) \Psi_{s}(x) d y d x d s .
$$

Proposition 2.1 Suppose $\left\{U_{M, n, K}: M, n, K \in \mathbb{N}, K \geq K_{1}\right\}$ are $\mathcal{F}_{t}$-stopping times such that for each $K \in \mathbb{N}^{\geq K_{1}}$,

$\left(H_{1}\right) \quad U_{M, n, K} \leq T_{K}, U_{M, n, K} \uparrow T_{K}$ as $M \rightarrow \infty$ for each $n$, and

$$
\lim _{M \rightarrow \infty} \sup _{n} P\left(U_{M, n, K}<T_{K}\right)=0,
$$

and

$\left(H_{2}\right) \quad$ For all $M \in \mathbb{N}, \lim _{n \rightarrow \infty} E\left(I^{n}\left(t_{0} \wedge U_{M, n, K}\right)\right)=0$.

Then the conclusion of Theorem 1.2 holds.

Proof. We adapt the reasoning in Lemma 2.2 of (MPS06) for the coloured noise setting to our white noise driven equation. As in (2.25) we have

$$
\text { for all } T, \lambda, p>0, \quad E\left(\sup _{0 \leq t \leq T} \sup _{x \in \mathbb{R}}|u(t, x)|^{p} e^{-\lambda|x|}\right)<\infty .
$$

Let

$$
Z_{n}(t)=\int \phi_{n}\left(\left\langle u_{t}, \Phi_{x}^{m_{n}}\right\rangle\right) \Psi_{t}(x) d x
$$

Fix $K \in \mathbb{N}^{\geq K_{1}}$ and $0 \leq t \leq t_{0}$. Note that since $0 \leq \phi_{n}(z) \leq|z|$ and $\Psi \geq 0$,

$$
\begin{aligned}
0 \leq Z_{n}\left(t \wedge T_{K}\right) & \leq \iint\left|u\left(t \wedge T_{K}, y\right)\right| \Phi_{x}^{m_{n}}(y) \Psi\left(t \wedge T_{K}, x\right) d y d x \\
& \leq 2 K \iint e^{|y|} \Phi_{x}^{m_{n}}(y) \Psi\left(t \wedge T_{K}, x\right) d y 1\left(|x| \leq K_{1}\right) d x \\
& \leq 2 K e^{K_{1}+1} c_{1}(\Psi) .
\end{aligned}
$$

With (2.41) in hand, the proof of Lemma 2.2(a) of (MPS06) is easily adapted (again it is in fact easier) to show

$$
\left\{I_{1}^{m_{n}, n}(s): s \leq t_{0}\right\} \text { is an } \mathbb{L}^{2} \text {-bounded sequence of } \mathbb{L}^{2} \text { martingales. }
$$


The proof of Lemma 2.2(b) of (MPS06) applies directly to show that

$$
I_{2}^{m_{n}, n}=I_{2}^{m_{n}, n, 1}+I_{2}^{m_{n}, n, 2},
$$

where for any stopping time $T$,

$$
I_{2}^{m_{n}, n, 1}(t \wedge T) \rightarrow \int_{0}^{t \wedge T} \int|u(s, x)| \frac{1}{2} \Delta \Psi_{s}(x) d x d s \text { in } \mathbb{L}^{1} \text { as } n \rightarrow \infty
$$

(again the key bound here is (2.41)), and we have the one-sided bound

$$
I_{2}^{m_{n}, n, 2}(s) \leq \frac{a_{n}}{n} C(\Psi) \text { for all } s \leq t_{0} \text { and } n .
$$

(In the notation of (MPS06) $), I_{2}^{m_{n}, n, 1}(s)=\int_{0}^{s} I_{2,3}^{m_{n}, n}(r) d r$ and $I_{2}^{m_{n}, n, 2}(s)=\int_{0}^{s} I_{2,1}^{m_{n}, n}(r)+I_{2,2}^{m_{n}, n}(r) d r$. ) The proof of Lemma 2.2(c) of (MPS06) also applies directly to show that for any stopping time $T$,

$$
I_{4}^{m_{n}, n}(t \wedge T) \rightarrow \int_{0}^{t \wedge T} \int|u(s, x)| \dot{\Psi}_{s}(x) d x d s \text { in } \mathbb{L}^{1} \text { as } n \rightarrow \infty .
$$

Since $\left|\phi_{n}^{\prime}\right| \leq 1$, (1.4) implies that for a stopping time $T$,

$$
I_{5}^{m_{n}, n}(t \wedge T) \leq B \int_{0}^{t \wedge T} \iint|u(s, y)| \Phi_{x}^{m_{n}}(y) \Psi_{s}(x) d y d x d s \equiv B \tilde{I}_{5}^{n}(t \wedge T) .
$$

It follows easily from (2.41) that $\left\{\tilde{I}_{5}^{n}\left(t_{0}\right): n \in \mathbb{N}\right\}$ is $\mathbb{L}^{2}$-bounded and, as $n \rightarrow \infty$,

$$
\tilde{I}_{5}^{n}(t \wedge T) \rightarrow \int_{0}^{t \wedge T} \int|u(s, x)| \Psi_{s}(x) d x d s \text { a.s. and hence in } \mathbb{L}^{1} \text { by the above. }
$$

Let $\varepsilon>0$. Then $\left(H_{1}\right),(2.42),(2.43),(2.44)$ and (2.46) show that, by a standard result for uniformly integrable random variables, there is an $M_{0}$ so that

$$
\begin{aligned}
\sup _{n} E\left(\left(\left|Z_{n}\left(t \wedge T_{K}\right)\right|+\left|I_{1}^{m_{n}, n}\left(t \wedge T_{K}\right)\right|\right.\right. & \left.+\left|I_{2}^{m_{n}, n, 1}\left(t \wedge T_{K}\right)\right|+\left|I_{4}^{m_{n}, n}\left(t \wedge T_{K}\right)\right|\right) \\
& \left.\times 1\left(U_{M, n, K}<T_{K}\right)\right)<\varepsilon \text { for all } M \geq M_{0} .
\end{aligned}
$$

From (2.37), (2.47), the non-negativity of $I_{3}^{m_{n}, n}$ and $\tilde{I}_{5}^{n}$, and Fatou's Lemma, we have for $M \geq M_{0}$,

$$
\begin{aligned}
& E\left(\int\left|u\left(t \wedge T_{K}, x\right)\right| \Psi_{t \wedge T_{K}}(x) d x\right) \\
& \leq \liminf _{n \rightarrow \infty} E\left(Z_{n}\left(t \wedge T_{K}\right) 1\left(U_{M, n, K}=T_{K}\right)\right)+E\left(Z_{n}\left(t \wedge T_{K}\right) 1\left(U_{M, n, K}<T_{K}\right)\right) \\
& \left.\leq \liminf _{n \rightarrow \infty} E\left(I_{1}^{m_{n}, n}\left(t \wedge T_{K}\right)\right)+E\left(I_{2}^{m_{n}, n, 1}\left(t \wedge T_{K}\right)\right)+E\left(I_{2}^{m_{n}, n, 2}\left(t \wedge T_{K}\right) 1\left(U_{M, n, K}\right)=T_{K}\right)\right) \\
& \quad+E\left(I_{3}^{m_{n}, n}\left(t \wedge U_{M, n, K}\right)\right)+E\left(I_{4}^{m_{n}, n}\left(t \wedge T_{K}\right)\right)+B E\left(\tilde{I}_{5}^{n}\left(t \wedge T_{K}\right)\right) \\
& \quad-E\left(I_{1}^{m_{n}, n}\left(t \wedge T_{K}\right) 1\left(U_{M, n, K}<T_{K}\right)\right)-E\left(I_{2}^{m_{n}, n, 1}\left(t \wedge T_{K}\right) 1\left(U_{M, n, K}<T_{K}\right)\right) \\
& \quad-E\left(I_{4}^{m_{n}, n}\left(t \wedge T_{K}\right) 1\left(U_{M, n, K}<T_{K}\right)\right)+E\left(Z_{n}\left(t \wedge T_{K}\right) 1\left(U_{M, n, K}<T_{K}\right)\right) \\
& \leq \liminf _{n \rightarrow \infty} E\left(\int_{0}^{t \wedge T_{K}} \int|u(s, x)| \frac{1}{2} \Delta \Psi_{s}(x) d x d s\right)+\frac{a_{n}}{n} C(\Psi) \\
& \quad+E\left(\int_{0}^{t \wedge T_{K}} \int|u(s, x)| \dot{\Psi}_{s}(x) d x d s\right)+B E\left(\int_{0}^{t \wedge T_{K}} \int|u(s, x)| \Psi_{s}(x) d x d s\right)+\varepsilon,
\end{aligned}
$$


by (2.43), (2.44), (2.45), $\left(H_{2}\right)$ (together with the bound (2.39) $),(2.46)(2.48)$, and (2.49), respectively. Let $\varepsilon \downarrow 0$ to see that

$$
E\left(\int\left|u\left(t \wedge T_{K}, x\right)\right| \Psi_{t \wedge T_{K}}(x) d x\right) \leq E\left(\int_{0}^{t \wedge T_{K}} \int|u(s, x)|\left(\frac{1}{2} \Delta \Psi_{s}(x)+\dot{\Psi}_{s}(x)+B \Psi_{s}(x)\right) d x d s\right) .
$$

Let $K \rightarrow \infty$ and use Dominated Convergence (recall (2.41) $)$ on each side to conclude that

$$
\int E(|u(t, x)|) \Psi_{t}(x) d x \leq \int_{0}^{t} \int E(|u(s, x)|)\left(\frac{1}{2} \Delta \Psi_{s}(x)+\dot{\Psi}_{s}(x)+B \Psi_{s}(x)\right) d x d s, \quad 0 \leq t \leq t_{0} .
$$

This gives (34) of (MPS06) with an additional drift term $B \Psi_{s}(x)$. One proceeds exactly as in Section 3 of that reference, using the semigroup $e^{B t} P_{t}$ in place of $P_{t}$, to see that since $E(|u(t, x)|)$ is a finite (by (2.41) ) non-negative subsolution of the heat equation with initial data zero, therefore $E(|u(t, x)|)=0$ and so $X^{1}=X^{2}$ by continuity of paths.

The construction of $\left\{U_{M, n, K}\right\}$ and verification of $\left(H_{1}\right)$ and $\left(H_{2}\right)$ will be the objective of the rest of this work.

Notation. For $t, t^{\prime} \geq 0$ and $x, x^{\prime} \in \mathbb{R}$ let $d\left((t, x),\left(t^{\prime}, x^{\prime}\right)\right)=\sqrt{\left|t^{\prime}-t\right|}+\left|x^{\prime}-x\right|$.

Note that the indicator function in the definition of $I^{n}$ implies there is an $\hat{x}_{0} \in\left(x-\sqrt{a}_{n}, x+\sqrt{a}_{n}\right)$ such that $\left|u\left(s, \hat{x}_{0}\right)\right| \leq a_{n}$. If we could take $\hat{x}_{0}=y$ we could bound $I_{n}(t)$ by $C(t) a_{n}^{-3 / 2-2 / n+2 \gamma}$, and $\left(H_{1}\right)$ and $\left(H_{2}\right)$ would follow immediately with $U_{M, n, K}=T_{K}$. (The criticality of $3 / 4$ in this argument is illusory as it follows from our choice of $m_{n}$.) The hypotheses of Proposition 2.1 now turn on getting good bounds on $\left|u(s, y)-u\left(s, \hat{x}_{0}\right)\right|$. The standard $1 / 2-\varepsilon$-Hölder modulus 1 not surprisingly, gives nothing. In (MPS06) this was refined to a $1-\varepsilon$-Hölder modulus near points where $u$ is small as we now describe. Let

$$
\begin{aligned}
& Z(N, K)(\omega)=\left\{(t, x) \in\left[0, T_{K}\right] \times[-K, K]: \text { there is a }\left(\hat{t}_{0}, \hat{x}_{0}\right) \in\left[0, T_{K}\right] \times \mathbb{R}\right. \text { such that } \\
&\left.d\left(\left(\hat{t}_{0}, \hat{x}_{0}\right),(t, x)\right) \leq 2^{-N}, \text { and }\left|u\left(\hat{t}_{0}, \hat{x}_{0}\right)\right| \leq 2^{-N}\right\} .
\end{aligned}
$$

Return now to the SPDE driven by coloured noise (1.9) from Section 1. Let $\tilde{u}$ be the difference of two solutions of (1.9) and $\alpha \in(0,2 \wedge d)$ be the covariance kernel exponent as in (1.8). Let $\tilde{Z}(N, K)$ be defined as $Z(N, K)$ with $\tilde{u}$ instead of $u$. The following improved modulus of continuity was proved in (MPS06) (see Theorem 4.1 and the first two paragraphs of the proof of Corollary 4.2 in that reference).

Theorem 2.2 For each $K \in \mathbb{N}$ and $0<\xi<\frac{1-\frac{\alpha}{2}}{1-\gamma} \wedge 1$ there is an $N_{0}=N_{0}(\xi, K, \omega) \in \mathbb{N}$ a.s. such that for all natural numbers $N \geq N_{0}$ and all $(t, x) \in \tilde{Z}(N, K)$,

$$
d\left(\left(t^{\prime}, x^{\prime}\right),(t, x)\right) \leq 2^{-N} \text { and } t^{\prime} \leq T_{K} \text { implies }\left|\tilde{u}\left(t^{\prime}, x^{\prime}\right)-\tilde{u}(t, x)\right| \leq 2^{-N \xi} .
$$

In the white noise setting the result holds with $\alpha=1$. Recall $u$ is the difference of two solutions to (1.1).

\footnotetext{
${ }^{1}$ Although this is well-known "folklore" result we were not able to find the exact reference. One can easily check that the estimates in the proof of Corollary 3.4 in (Wal86) give $1 / 2-\varepsilon$-Hölder spatial modulus; similarly the result of (SS02) can be immediately extended to cover the white noise case; in both works the Lipschitz assumptions on noise coefficients can be relaxed to linear growth assumptions and the proofs still go through.
} 
Theorem 2.3 Assume $\gamma \geq 1 / 2$. For each $K \in \mathbb{N}$ and $\xi \in(0,1)$ there is an $N_{0}=N_{0}(\xi, K, \omega) \in \mathbb{N}$ a.s. such that for all natural numbers $N \geq N_{0}$ and all $(t, x) \in Z(N, K)$,

$$
d\left(\left(t^{\prime}, x^{\prime}\right),(t, x)\right) \leq 2^{-N} \text { and } t^{\prime} \leq T_{K} \text { implies }\left|u\left(t^{\prime}, x^{\prime}\right)-u(t, x)\right| \leq 2^{-N \xi} .
$$

Proof. The proof of Theorem 2.2 applies with $\alpha=1$ (the dependance of $\sigma$ on $t, x$ alters nothing in the proof). In fact it is now considerably simpler because of the orthogonality of white noise increments. The required tools are Lemma 4.1 and Lemma 4.3 below. With this choice of $\alpha$ and $\gamma \geq 1 / 2$, the upper bound on $\xi$ in Theorem 2.2 becomes 1. In (MPS06) there was no drift term, but the calculations for the Lipschitz drift term are simpler still. Here one uses Hölder's inequality to utilize the $\mathbb{L}^{2}$ bounds in Lemma 4.3 .

The proof of Theorem 1.2 is long and involved so before descending into the technical details of the derivation of $\left(H_{1}\right)$ and $\left(H_{2}\right)$, we now give a heuristic description of the method with $b \equiv 0$ throughout, and also try to explain why $\gamma=3 / 4$ is critical in our approach. The choice of $m_{n}=a_{n-1}^{-1 / 2}$ appears arbitrarily in the above so let us for the moment set $m_{n}=a_{n-1}^{-\alpha_{0}} \approx a_{n}^{-\alpha_{0}}$ for some $\alpha_{0}>0$. $\left(H_{1}\right)$ and $\left(H_{2}\right)$ are delicate ways of ensuring $I^{n}(t)$ approaches zero as $n \rightarrow \infty$ and so our goal is to show that

$$
\begin{aligned}
I^{n}(t) & \approx a_{n}^{-1-\alpha_{0}} \int_{0}^{t} \iint 1\left(\left|\left\langle u_{s}, \Phi_{x}^{m_{n+1}}\right\rangle\right|<a_{n}\right)|u(s, y)|^{2 \gamma} \Phi_{x}^{m_{n+1}}(y) \Psi_{s}(x) d y d x d s \\
& \rightarrow 0 \text { as } \mathrm{n} \rightarrow \infty
\end{aligned}
$$

We have taken (and will take) some small liberties with the "local time term" $I^{n}(t)$ (with this new choice of $\left.m_{n}\right)$ in the first line. In the integrand in (2.50) the variable $y$ must be within $2 a_{n}^{\alpha_{0}}$ of a point $\hat{x}_{0}$ where $\left|u\left(s, \hat{x}_{0}\right)\right|<a_{n}$. If we simply replace $y$ with $\hat{x}_{0}, I^{n}(t)$ is at most

$$
a_{n}^{-1-\alpha_{0}} \int_{0}^{t} \iint a_{n}^{2 \gamma} \Phi_{x}^{m_{n+1}}(y) \Psi_{s}(x) d y d x d s \leq C t a_{n}^{2 \gamma-1-\alpha_{0}} \rightarrow 0
$$

if $\gamma>1 / 2$ and $\alpha_{0}$ is small enough. This is a bit too crude but shows it will be crucial to get good estimates on $u(s, \cdot)$ near points where it is small (and also shows we are already forced to assume $\gamma>1 / 2)$. Theorem 2.3 implies that

$$
\gamma \geq 1 / 2 \text { implies } u(t, \cdot) \text { is } \xi \text {-Hölder continuous near its zero set for } \xi<1 \text {, }
$$

and so allows us to bound $\left|u(s, y)-u\left(s, \hat{x}_{0}\right)\right|$. Use this in (2.50) and take $0<\alpha_{0} \leq 1$ to bound $I^{n}(t)$ by

$$
\begin{array}{r}
a_{n}^{-1-\alpha_{0}} \int_{0}^{t} \iint C a_{n}^{\alpha_{0} \xi 2 \gamma} \Phi_{x}^{m_{n+1}}(y) \Psi_{s}(x) d y d x d s \\
\leq C t a_{n}^{-1+\alpha_{0}(\xi 2 \gamma-1)} \rightarrow 0 \text { as } n \rightarrow \infty
\end{array}
$$

if $\gamma>1$ and we choose $\alpha_{0}, \xi$ close to one. Of course $\gamma>1$ is not a viable choice but this shows we are now getting close, and in fact in the coloured noise setting of (MPS06) the above argument 
sufficed for the results there, although there was some work to be done to implement this idea carefully.

To increase our control on $u(s, \cdot)$ near its zero set we will improve (2.51) to

$$
\gamma>3 / 4 \text { implies } u^{\prime}(s, \cdot) \text { is } \xi \text {-Hölder on }\left\{x: u(s, x) \approx u^{\prime}(s, x) \approx 0\right\} \text { for } \xi<1 \text {, }
$$

where $u^{\prime}$ denotes the spatial derivative. Corollary 5.9 below with $m=\bar{m}+1$ is the closest result which comes to a formal statement of the above, although the condition on $\gamma$ is implicit.

We first make the case that for $\gamma<3 / 4$, we cannot expect the following slight strengthening of (2.52):

$$
u(s, \cdot) \text { is } C^{2} \text { on }\left\{x: u(s, x) \approx u^{\prime}(s, x) \approx 0\right\} .
$$

A formal differentiation of (1.5) (recall $b \equiv 0$ and $u$ is the difference of the $X^{i}$ 's) gives for $u(t, x) \approx$ $u^{\prime}(t, x) \approx 0$,

$$
u^{\prime \prime}(t, x)=\int_{0}^{t} \int p_{t-s}^{\prime \prime}(y-x)\left[\sigma\left(s, y, X^{1}(s, y)\right)-\sigma\left(s, y, X^{2}(s, y)\right)\right] W(d s, d y) .
$$

If $\sigma$ is a Weierstrass-type function that realizes its Hölder modulus at typical points we have

$$
\left|\sigma\left(s, y, X^{1}(s, y)\right)-\sigma\left(s, y, X^{2}(s, y)\right)\right| \approx L|u(s, y)|^{\gamma},
$$

and for $s<t$ and very close to $t$, we have by a Taylor series expansion in space,

$$
|u(s, y)| \approx\left|u^{\prime \prime}(s, x)\right| \frac{(y-x)^{2}}{2} .
$$

Use these approximations in the finite square function associated with the right hand side of (2.54) and conclude that

$$
\begin{aligned}
\infty & >\int_{t-\delta}^{t} \int p_{t-s}^{\prime \prime}(y-x)^{2}\left[\frac{\left|u^{\prime \prime}(s, x)\right|(y-x)^{2}}{2}\right]^{2 \gamma} d y d s \\
& \approx c\left|u^{\prime \prime}(t, x)\right|^{2 \gamma} \int_{t-\delta}^{t} \int p_{t-s}(z)^{2}\left[z^{2}(t-s)^{-2}-(t-s)^{-1}\right]^{2} z^{4 \gamma} d z d s \\
& \approx c\left|u^{\prime \prime}(t, x)\right|^{2 \gamma} \int_{t-\delta}^{t}(t-s)^{2 \gamma-\frac{5}{2}} d s,
\end{aligned}
$$

which implies $\gamma>3 / 4$.

We next show how (2.52) will lead to (2.50). Taking further liberties with $I^{n}(t)$ and recalling $m_{n} \approx a_{n}^{\alpha_{0}}$, we get

$$
\begin{aligned}
I^{n}(t) & \approx a_{n}^{-1-\alpha_{0}} \sum_{\beta} \int_{0}^{t} \iint 1\left(\left.\left|u\left(s, x \mid \leq a_{n}, u^{\prime}(s, x) \approx \pm a_{n}^{\beta}\right)\right| u(s, y)\right|^{2 \gamma} \Phi_{x}^{m_{n+1}}(y) \Psi_{s}(x) d y d x d s\right. \\
& \equiv \sum_{\beta} I_{\beta}^{n}(t)
\end{aligned}
$$


where $\sum_{\beta}$ indicates we are summing over a finite grid $\beta_{i} \in[0, \bar{\beta}]$ ( $\bar{\beta}$ to be determined below) and are bounding $u^{\prime}(s, x)$ in the appropriate grid interval and its mirror image in the origin. As the sum is finite we may fix $\beta \in(0, \bar{\beta}]$ and consider only $u^{\prime}(s, x) \approx a_{n}^{\beta}$. The value $\beta=0$ is a bit special but should be clear from the argument below. A Taylor series expansion and (2.52) with $\xi \approx 1$ show that for $y$ as in the integrand of $I_{\beta}^{n}(t)$,

$$
\begin{aligned}
|u(s, y)| & \leq|u(s, x)|+\left(\left|u^{\prime}(s, x)\right|+L|y-x|^{\xi}\right)|y-x| \\
& \leq a_{n}+a_{n}^{\beta+\alpha_{0}}+L a_{n}^{\alpha_{0}(\xi+1)} \\
& \leq C a_{n}^{\left(\frac{\xi}{2} \wedge \beta\right)+\frac{1}{2}}
\end{aligned}
$$

where a comparison of the first and last terms in the second line leads naturally to $\alpha_{0}=1 / 2$. Substitute this into the integrand of $I_{\beta}^{n}$, integrate out $y$, and conclude

$$
I_{\beta}^{n}(t) \leq C a_{n}^{\frac{-3}{2}+\gamma+\gamma(2 \beta \wedge \xi)} \int_{0}^{t} \int 1\left(|u(s, x)| \leq a_{n}, u^{\prime}(s, x) \approx a_{n}^{\beta}\right) \Psi_{s}(x) d x d s .
$$

For $\beta=\bar{\beta}$ the precise meaning of $u^{\prime}(s, x) \approx a_{n}^{\bar{\beta}}$ is $0 \leq u^{\prime}(s, x) \leq a_{n}^{\bar{\beta}}$ and we have from (2.56),

$$
I_{\bar{\beta}}^{n}(t) \leq C t a_{n}^{\frac{-3}{2}}+\gamma+\gamma(2 \bar{\beta} \wedge \xi) .
$$

Consider $0<\beta<\bar{\beta}$. Recall that $\left\{x: \Psi_{s}(x)>0\right.$ for some $\left.s \leq t_{0}\right\} \subset\left[-K_{1}, K_{1}\right]$, let

$$
S_{n}(s)=\left\{x \in\left[-K_{1}, K_{1}\right]:|u(s, x)| \leq a_{n}, u^{\prime}(s, x) \geq a_{n}^{\beta}\right\}
$$

and $\left|S_{n}(s)\right|$ denote the Lebesgue measure of $S_{n}(s)$. From (2.52) we see that if $x \in S_{n}(s)$, then $u^{\prime}(s, y) \geq \frac{a_{n}^{\beta}}{2}$ if $|y-x| \leq L^{-1} a_{n}^{\beta / \xi}$, and so by the Fundamental Theorem of Calculus,

$$
u(s, y)>a_{n} \text { if } 4 a_{n}^{1-\beta}<|y-x| \leq L^{-1} a_{n}^{\beta / \xi} .
$$

A simple covering argument now shows that $\left|S_{n}(s)\right| \leq c\left(L, K_{1}\right) a_{n}^{1-\beta} a_{n}^{-\beta / \xi}$ and (2.56) implies

$$
\begin{aligned}
I_{\beta}^{n}(t) & \leq \operatorname{Cta}_{n}^{\frac{-3}{2}+\gamma+\gamma(2 \beta \wedge \xi)+1-\beta-\frac{\beta}{\xi}} \\
& \leq \operatorname{Cta}_{n}^{\gamma(1+(2 \bar{\beta}) \wedge \xi)-\frac{1}{2}-\bar{\beta}\left(1+\frac{1}{\xi}\right)} .
\end{aligned}
$$

So from (2.57) and (2.58) we see that $\lim _{n \rightarrow \infty} I_{\beta}^{n}(t)=0$ will follow for all $\beta \leq \bar{\beta}$ if

$$
\gamma(1+(2 \bar{\beta} \wedge 1))>\frac{3}{2} \quad \text { and } \quad \gamma(1+(2 \bar{\beta} \wedge 1))>\frac{1}{2}+2 \bar{\beta},
$$

that is, $\gamma>(1+(2 \bar{\beta} \wedge 1))^{-1}\left(\frac{3}{2} \vee\left(\frac{1}{2}+2 \bar{\beta}\right)\right)$. The right-hand side is minimized when $\bar{\beta}=\frac{1}{2}$, and leads to $\gamma>\frac{3}{4}$, as required, and also establishes the range $0 \leq \beta \leq \frac{1}{2}$, which will be used below.

The above heuristics show that $\gamma>3 / 4$ and the regularity of $u$ given in (2.52) (or (2.53) ) is optimal for our approach. If we try weakening the regularity condition on $u$, the above discussion shows we would have to increase $3 / 4$ to show that $I^{n}(t) \rightarrow 0$. The earlier discussion shows that a strengthening of the regularity on $u$ would require increasing $3 / 4$ as well. 
A major obstruction to (2.52) is the fact that we cannot expect $u^{\prime}(s, x)$ to exist as soon as $u(s, x) \neq 0$ (and don't even know this is the case for $u(s, x)=0)$. So instead, if $D(r, y)=$ $\sigma\left(r, y, X^{1}(r, y)\right)-\sigma\left(r, y, X^{2}(r, y)\right)$, then we will use (1.5) to decompose $u$ as

$$
\begin{aligned}
u(t, x) & =\int_{0}^{t-a_{n}} \int p_{t-r}(y-x) D(r, y) W(d r, d y)+\int_{t-a_{n}}^{t} \int p_{t-r}(y-x) D(r, y) W(d r, d y) \\
& \equiv u_{1, a_{n}}(t, x)+u_{2, a_{n}}(t, x) .
\end{aligned}
$$

$u_{1, a_{n}}$ is smooth in the spatial variable and so the above arguments may be applied with $u_{1, a_{n}}^{\prime}(t, x)$ playing the role of $u^{\prime}(t, x)$, while $u_{2, a_{n}}$ and its increments should lead to small and manageable error terms. Proposition 5.14 gives the required bounds on the increments of $u_{2, a_{n}^{\alpha}}$, and (as noted above) Corollary [5.9 is the analogue of (2.52) for $u_{1, a_{n}^{\alpha}}^{\prime}(\alpha \in[0,1])$. (The reason for the extension to $a_{n}^{\alpha}$ is discussed below.) The proofs of these results are incorporated into an inductive proof of a space-time bound $\left(P_{m}\right)$ for $u(t, x)$ when $(t, x)$ is close to a point $\left(\hat{t}_{0}, \hat{x}_{0}\right)$ where

$$
\left|u\left(\hat{t}_{0}, \hat{x}_{0}\right)\right| \leq a_{n} \text { and }\left|u_{1, a_{n}^{\alpha}}^{\prime}\left(\hat{t}_{0}, \hat{x}_{0}\right)\right| \leq a_{n}^{\beta} .
$$

If

$$
d=\sqrt{\left|t-\hat{t}_{0}\right|}+\left|x-\hat{x}_{0}\right|
$$

then, roughly speaking, $\left(P_{m}\right)$ bounds $|u(t, x)|$ by

$$
d^{\xi}\left[d^{\tilde{\gamma}_{m-1}}+a_{n}^{\beta}\right]
$$

where $\tilde{\gamma}_{m}$ increases in $m$ and equals 2 for $m$ large, and $\xi<1$ as usual. When $\tilde{\gamma}_{m}=2$ this does capture the kind of bound one expects from (2.52). The reader may find a precise statement of $\left(P_{m}\right)$ prior to Proposition 5.1 (the statement of its validity).

The $m=0$ case will be an easy consequence of our improved local modulus of continuity, Theorem 2.3. Note that (2.29) ) implies

$$
|D(r, y)| \leq R_{0} e^{R_{1}|y|}|u(r, y)|^{\gamma} .
$$

The inductive proof of $\left(P_{m}\right)$ proceeds by using (2.63) and then (2.62) to bound the square functions associated with the space-time increments of $u_{1, a_{n}^{\alpha}}^{\prime}$ and $u_{2, a_{n}^{\alpha}}$ for points near $\left(\hat{t}_{0}, \hat{x}_{0}\right)$ as in (2.60) (recall (2.59)). These give good control of the integrands of these square functions near the points where they have singularities. This will then lead to Corollary 5.9] and Proposition 5.14, our " $m$ th order" bounds for the increments of $u_{1, a_{n}^{\alpha}}^{\prime}$ and $u_{2, a_{n}^{\alpha}}$. We then use the slightly generalized version of (2.59),

$$
u(t, x)=u_{1, a_{n}^{\alpha}}(t, x)+u_{2, a_{n}^{\alpha}}(t, x)
$$

to derive $\left(P_{m+1}\right)$. At this point we will optimize over $\alpha$ since decreasing $\alpha$ increases the regularity of $u_{1, a_{n}^{\alpha}}$ but increases the size of the error term $u_{2, a_{n}^{\alpha}}$. The optimal choice will be so that $a_{n}^{\alpha} \approx d$, where $d$ is as in (2.61).

There are at least two issues to address here. First, how do you control $u_{1, a_{n}^{\alpha}}^{\prime}(t, x)$ when all you know is $\left|u_{1, a_{n}}^{\prime}(t, x)\right| \leq a_{n}^{\beta}$ ? Second, how do you control the time increments of $u_{1, a_{n}^{\alpha}}$ when you only have good estimates on the spatial derivatives? The first question is answered in Proposition 5.11 
which will give surprisingly good bounds on $\left|u_{1, a_{n}^{\alpha}}^{\prime}\left(\hat{t}_{0}, \hat{x}_{0}\right)-u_{1, a_{n}}^{\prime}\left(\hat{t}_{0}, \hat{x}_{0}\right)\right|$. The second question is answered in Proposition [5.13, where the key step is to note (see (5.70) ) that for $t>t^{\prime}$,

$$
\left|u_{1, a_{n}^{\alpha}}(t, x)-u_{1, a_{n}^{\alpha}}\left(t^{\prime}, x\right)\right| \approx\left|P_{t-t^{\prime}}\left(u_{1, a_{n}^{\alpha}}\left(t^{\prime}, \cdot\right)\right)(x)-u_{1, a_{n}^{\alpha}}\left(t^{\prime}, x\right)\right|,
$$

where $P_{t}$ is the Brownian semigroup. The fact that the Brownian semigroup, $P_{t} f$, inherits temporal regularity from spatial regularity of $f$ will give the required regularity in time.

A critical step in the above argument was finding a form of $\left(P_{m}\right)$ which actually iterates to produce $\left(P_{m+1}\right)$. Note also that although the required bound on $I_{\beta}^{n}(t)$ (see (2.56)) only required good spatial estimates for $u(s, \cdot)$ near points $(s, x)=\left(\hat{t}_{0}, \hat{x}_{0}\right)$ as in (2.60), the iteration of estimates requires an expansion in both space and time.

Turning now to a brief description of the contents of the paper, we first set $b \equiv 0$. In Section 3 we reduce $\left(H_{1}\right)$ and $\left(H_{2}\right)$ to a result (Proposition 3.3) on control of the spatial increments of $u_{2, a_{n}^{\alpha}}$ and size of $u_{1, a_{n}^{\alpha}}^{\prime}$ on relatively long intervals near a spatial point where $\left|u\left(s, \hat{x}_{0}\right)\right|$ is small and $u_{1, a_{n}^{\alpha}}^{\prime}\left(s, \hat{x}_{0}\right) \approx a_{n}^{\beta}$. This includes the covering argument sketched above. Section 4 gives some integral bounds for heat kernels and their derivatives which will help bound the square functions

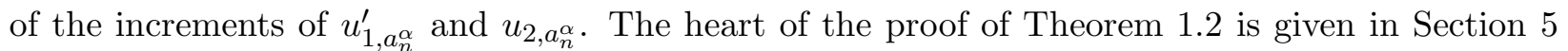
where the inductive proof of $\left(P_{m}\right)$ is given. As was sketched above, this argument includes good local expansions for $u_{1, a_{n}^{\alpha}}^{\prime}$ and $u_{2, a_{n}^{\alpha}}$ near points where $|u|$ and $\left|u_{1, a_{n}^{\alpha}}^{\prime}\right|$ are small, although the (easier) proof for $u_{2, a_{n}^{\alpha}}$ is deferred until Section 7. These expansions, with $m$ large enough, are then used in Section 6 to prove Proposition 3.3 and so complete the proof of Theorem 1.2 for $b \equiv 0$. In Section 8 we describe the relatively simple additions that are needed to include a Lipschitz drift $b$ in the argument already presented.

\section{Verification of the Hypotheses of Proposition 2.1}

We assume throughout this Section that $b \equiv 0$-the relatively simple refinements required to include the drift are outlined in Section 8. Let $X^{1}, X^{2}$ be as in Section 2, $u=X^{1}-X^{2}$, and assume the hypotheses of Theorem 1.2 as well as (2.29). If $D(s, y)=\sigma\left(s, y, X^{1}(s, y)\right)-\sigma\left(s, y, X^{2}(s, y)\right)$, then

$$
u(t, x)=\int_{0}^{t} \int p_{t-s}(y-x) D(s, y) W(d s, d y) \text { a.s. for all }(t, x),
$$

and by (2.29),

$$
|D(s, y)| \leq R_{0} e^{R_{1}|y|}|u(s, y)|^{\gamma} .
$$

$\delta$ will always take values in $(0,1]$. Let

$$
u_{1, \delta}(t, x)=P_{\delta}\left(u_{(t-\delta)^{+}}\right)(x) \text { and } u_{2, \delta}(t, x)=u(t, x)-u_{1, \delta}(t, x) .
$$

Since $P_{\delta}: C_{t e m} \rightarrow C_{t e m}$ is uniformly continuous (by Lemma $6.2\left(\right.$ ii) of (Shi94)), $u_{1, \delta}$ and $u_{2, \delta}$ both have sample paths in $C\left(\mathbb{R}, C_{t e m}\right)$. (3.1) implies that

$$
u_{1, \delta}(t, x)=\int\left[\int_{0}^{(t-\delta)^{+}} \int p_{(t-\delta)^{+-s}}(y-z) D(s, y) W(d s, d y)\right] p_{\delta}(z-x) d z .
$$


A stochastic Fubini argument (Theorem 2.6 of (Wal86)) then gives

$$
u_{1, \delta}(t, x)=\int_{0}^{(t-\delta)^{+}} \int p_{t-s}(y-x) D(s, y) W(d s, d y) \text { a.s. for all }(t, x) \in \mathbb{R}_{+} \times \mathbb{R} .
$$

(The above identity is trivial for $t \leq \delta$ since $u(0, \cdot) \equiv 0$.) The expectation condition in Walsh's Theorem 2.6 may be realized by localization with the stopping times $\left\{T_{K}\right\}$, working with $D\left(s \wedge T_{K}\right)$, and letting $K \rightarrow \infty$. It follows that

$$
u_{2, \delta}(t, x)=\int_{(t-\delta)^{+}}^{t} \int p_{t-s}(y-x) D(s, y) W(d s, d y) \text { a.s. for all }(t, x) \in \mathbb{R}_{+} \times \mathbb{R} .
$$

Hence $u_{j, \delta}(j=1,2)$ define jointly continuous versions of the right-hand sides of (3.4) and (3.5).

Notation. If $s, t \geq 0$ and $x \in \mathbb{R}$, let $G_{\delta}(s, t, x)=P_{(t-s)^{++} \delta}\left(u_{(s-\delta)^{+}}\right)(x)$ and $F_{\delta}(s, t, x)=-\frac{d}{d x} G_{\delta}(s, t, x) \equiv-G_{\delta}^{\prime}(s, t, x)$, if the derivative exists.

Lemma 3.1 $G_{\delta}^{\prime}(s, t, x)$ exists for all $(s, t, x) \in \mathbb{R}_{+}^{2} \times \mathbb{R}$, is jointly continuous in $(s, t, x)$, and satisfies

$$
F_{\delta}(s, t, x)=\int_{0}^{(s-\delta)^{+}} \int p_{(t \vee s)-r}^{\prime}(y-x) D(r, y) W(d r, d y) \text { for all } s \text { a.s. for all }(t, x) .
$$

Proof. Since $G_{\delta}(s, t, x)=\int p_{(t-s)^{+}+\delta}(y-x) u\left((s-\delta)^{+}, y\right) d y$ and

$$
\sup _{s \leq T, y} e^{-|y|}\left|u\left((s-\delta)^{+}, y\right)\right|<\infty \text { for all } T>0 \text { a.s., }
$$

a simple Dominated Convergence argument shows that

$$
G_{\delta}^{\prime}(s, t, x)=-\int p_{(t-s)^{+}+\delta}^{\prime}(y-x) u\left((s-\delta)^{+}, y\right) d y \quad \text { for all }(s, t, x) \text { a.s. }
$$

Another application of (3.7) and Dominated Convergence gives the a.s. joint continuity of the right-hand side of (3.8), and hence of $G_{\delta}^{\prime}$.

To prove (3.6) we may assume without loss of generality that $t \geq s>\delta$. From (3.8) and (3.1) we have w.p. 1 ,

$$
G_{\delta}^{\prime}(s, t, x)=-\int p_{t-s+\delta}^{\prime}(y-x)\left[\int_{0}^{s-\delta} \int p_{s-\delta-r}(z-y) D(r, z) W(d r, d z)\right] d y \text { a.s. }
$$

Now use the stochastic Fubini theorem, as in the derivation of (3.4) above, to see that

$$
\begin{aligned}
G_{\delta}^{\prime}(s, t, x) & =-\int_{0}^{s-\delta} \int\left[\int p_{t-s+\delta}^{\prime}(y-x) p_{s-\delta-r}(z-y) d y\right] D(r, z) W(d r, d z) \\
& =-\int_{0}^{s-\delta} \int p_{t-r}^{\prime}(z-x) D(r, z) W(d r, d z) \quad \text { a.s. }
\end{aligned}
$$

In the last line we have used Dominated Convergence yet again to differentiate through the integral in the Chapman-Kolmorgorov equation. As both sides of (3.6) are continuous in $s$ we may take the null set to be independent of $s$. 
Remark 3.2 Since $G_{\delta}(t, t, x)=u_{1, \delta}(t, x)$, as a special case of the above we see that $u_{1, \delta}^{\prime}(t, x)$ is a.s. jointly continuous and satisfies

$$
u_{1, \delta}^{\prime}(t, x)=-\int_{0}^{(t-\delta)^{+}} \int p_{t-s}^{\prime}(y-x) D(s, y) W(d s, d y) \quad \text { a.s. for all }(t, x) .
$$

Definition. For $(t, x) \in \mathbb{R}_{+} \times \mathbb{R}$,

$$
\begin{aligned}
\hat{x}_{n}(t, x)(\omega) & =\inf \left\{y \in\left[x-\sqrt{a_{n}}, x+\sqrt{a_{n}}\right]:|u(t, y)|=\inf \left\{|u(t, z)|:|z-x| \leq \sqrt{a_{n}}\right\}\right\} \\
& \in\left[x-\sqrt{a_{n}}, x+\sqrt{a_{n}}\right] .
\end{aligned}
$$

It is easy to use the continuity of $u$ to check that $\hat{x}_{n}$ is well-defined and $\mathcal{B}\left(\mathbb{R}_{+} \times \mathbb{R}\right) \times \mathcal{F}$-measurable.

We fix a $K_{0} \in \mathbb{N}^{\geq K_{1}}$ and positive constants satisfying

$$
0<\varepsilon_{1}<\frac{1}{100}\left(\gamma-\frac{3}{4}\right), 0<\varepsilon_{0}<\frac{\varepsilon_{1}}{100} .
$$

We introduce a grid of $\beta$ values by setting

$$
L=L\left(\varepsilon_{0}, \varepsilon_{1}\right)=\left\lfloor\left((1 / 2)-6 \varepsilon_{1}\right) / \varepsilon_{0}\right\rfloor,
$$

and

$$
\begin{aligned}
\beta_{i} & =i \varepsilon_{0} \in\left[0, \frac{1}{2}-6 \varepsilon_{1}\right], \quad \alpha_{i}=2\left(\beta_{i}+\varepsilon_{1}\right) \in[0,1], \quad i=0, \ldots, L, \\
\beta_{L+1} & =\frac{1}{2}-\varepsilon_{1} .
\end{aligned}
$$

Note that $\beta=\beta_{i}, i=0, \ldots, L+1$, satisfies

$$
0 \leq \beta \leq \frac{1}{2}-\varepsilon_{1}
$$

If $s \geq 0$ set

$$
\begin{gathered}
J_{n, 0}(s)=\left\{x:|x| \leq K_{0},\left|\left\langle u_{s}, \Phi_{x}^{m_{n+1}}\right\rangle\right| \leq a_{n}, u_{1, a_{n}}^{\prime}\left(s, \hat{x}_{n}(s, x)\right) \geq \frac{a_{n}^{\varepsilon_{0}}}{4}\right\}, \\
J_{n, L}(s)=\left\{x:|x| \leq K_{0},\left|\left\langle u_{s}, \Phi_{x}^{m_{n+1}}\right\rangle\right| \leq a_{n}, u_{1, a_{n}}^{\prime}\left(s, \hat{x}_{n}(s, x)\right) \in\left[0, \frac{a_{n}^{\beta_{L}}}{4}\right]\right\},
\end{gathered}
$$

and for $i=1, \ldots, L-1$ set

$$
J_{n, i}(s)=\left\{x:|x| \leq K_{0},\left|\left\langle u_{s}, \Phi_{x}^{m_{n+1}}\right\rangle\right| \leq a_{n}, u_{1, a_{n}}^{\prime}\left(s, \hat{x}_{n}(s, x)\right) \in\left[\frac{a_{n}^{\beta_{i+1}}}{4}, \frac{a_{n}^{\beta_{i}}}{4}\right]\right\} .
$$

If $t_{0}>0$ is as in $\left(H_{2}\right)$ and $i=0, \ldots, L$, define

$$
J_{n, i}=\left\{(s, x): 0 \leq s, x \in J_{n, i}(s)\right\},
$$

and if $0 \leq t \leq t_{0}$, let

$$
I_{i}^{n}(t)=a_{n}^{-\frac{3}{2}-\frac{2}{n}} \int_{0}^{t} \iint 1_{J_{n, i}(s)}(x)|u(s, y)|^{2 \gamma} \Phi_{x}^{m_{n+1}}(y) \Psi_{s}(x) d y d x d s .
$$


Let

$$
\begin{array}{r}
I_{+}^{n}(t)=a_{n}^{-\frac{3}{2}-\frac{2}{n}} \int_{0}^{t} \iint 1\left(u_{1, a_{n}}^{\prime}(\right. \\
\left.\left(s, \hat{x}_{n}(s, x)\right) \geq 0\right) 1\left(\left|\left\langle u_{s}, \Phi_{x}^{m_{n+1}}\right\rangle\right|<a_{n}\right) \\
\times|u(s, y)|^{2 \gamma} \Phi_{x}^{m_{n+1}}(y) \Psi_{s}(x) d y d x d s .
\end{array}
$$

Then to prove Proposition 2.1 it suffices to construct the stopping times $\left\{U_{M, n} \equiv U_{M, n, K_{0}}: M, n \in\right.$ $\mathbb{N}$ \} satisfying $\left(H_{1}\right)$ such that

$$
\text { for each } M \in \mathbb{N}, \lim _{n \rightarrow \infty} E\left(I_{+}^{n}\left(t_{0} \wedge U_{M, n}\right)\right)=0 \text {. }
$$

Note that (3.13) implies $\left(H_{2}\right)$ by symmetry (interchange $X_{1}$ and $X_{2}$ ).

Our definitions imply

$$
I_{+}^{n}(t) \leq \sum_{i=0}^{L} I_{i}^{n}(t) \text { for all } t \leq t_{0},
$$

and so to prove $\left(H_{2}\right)$ it suffices to show that for $i=0, \ldots, L$,

$$
\text { for all } M \in \mathbb{N}, \quad \lim _{n \rightarrow \infty} E\left(I_{i}^{n}\left(t_{0} \wedge U_{M, n}\right)\right)=0 .
$$

Notation. $\overline{l_{n}}(\beta)=a_{n}^{\beta+5 \varepsilon_{1}}$.

Now introduce the related sets:

$$
\begin{aligned}
& \tilde{J}_{n, 0}(s)=\left\{x \in\left[-K_{0}, K_{0}\right]:\left|\left\langle u_{s}, \Phi_{x}^{m_{n+1}}\right\rangle\right| \leq a_{n}, u_{1, a_{n}^{\alpha_{0}}}^{\prime}\left(s, x^{\prime}\right) \geq a_{n}^{\beta_{1}} / 16\right. \\
& \text { for all } x^{\prime} \in\left[x-5 \overline{l_{n}}\left(\beta_{0}\right), x+5 \overline{l_{n}}\left(\beta_{0}\right)\right] \text {, } \\
& \left|u_{2, a_{n}^{\alpha_{0}}}\left(s, x^{\prime}\right)-u_{2, a_{n}^{\alpha_{0}}}\left(s, x^{\prime \prime}\right)\right| \leq 2^{-75} a_{n}^{\beta_{1}}\left(\left|x^{\prime}-x^{\prime \prime}\right| \vee a_{n}^{\gamma-2 \beta_{0}(1-\gamma)-\varepsilon_{1}}\right) \\
& \text { for all } x^{\prime} \in\left[x-4 \sqrt{a_{n}}, x+4 \sqrt{a_{n}}, x^{\prime \prime} \in\left[x^{\prime}-\overline{l_{n}}\left(\beta_{0}\right), x^{\prime}+\overline{l_{n}}\left(\beta_{0}\right)\right]\right. \text {, } \\
& \text { and } \left.\left|u\left(s, x^{\prime}\right)\right| \leq 3 a_{n}^{\left(1-\varepsilon_{0}\right) / 2} \text { for all } x^{\prime} \in\left[x-\sqrt{a_{n}}, x+\sqrt{a_{n}}\right]\right\} \text {, } \\
& \tilde{J}_{n, L}(s)=\left\{x \in\left[-K_{0}, K_{0}\right]:\left|\left\langle u_{s}, \Phi_{x}^{m_{n+1}}\right\rangle\right| \leq a_{n},\left|u_{1, a_{n}^{\prime}}^{\prime}\left(s, x^{\prime}\right)\right| \leq a_{n}^{\beta_{L}}\right. \\
& \text { for all } x^{\prime} \in\left[x-5 \overline{l_{n}}\left(\beta_{L}\right), x+5 \overline{l_{n}}\left(\beta_{L}\right)\right] \text {, } \\
& \text { and }\left|u_{2, a_{n}^{\alpha}}\left(s, x^{\prime}\right)-u_{2, a_{n}^{\alpha}}\left(s, x^{\prime \prime}\right)\right| \leq 2^{-75} a_{n}^{\beta_{L+1}}\left(\left|x^{\prime}-x^{\prime \prime}\right| \vee a_{n}^{\gamma-2 \beta_{L}(1-\gamma)-\varepsilon_{1}}\right) \\
& \text { for all } \left.x^{\prime} \in\left[x-4 \sqrt{a_{n}}, x+4 \sqrt{a_{n}}\right], x^{\prime \prime} \in\left[x^{\prime}-\overline{l_{n}}\left(\beta_{L}\right), x^{\prime}+\overline{l_{n}}\left(\beta_{L}\right)\right]\right\} \text {, }
\end{aligned}
$$

and for $i \in\{1, \ldots, L-1\}$,

$$
\begin{aligned}
& \tilde{J}_{n, i}(s)=\left\{x \in\left[-K_{0}, K_{0}\right]:\left|\left\langle u_{s}, \Phi_{x}^{m_{n+1}}\right\rangle\right| \leq a_{n}, u_{1, a_{n}^{\alpha_{i}}}^{\prime}\left(s, x^{\prime}\right) \in\left[a_{n}^{\beta_{i+1}} / 16, a_{n}^{\beta_{i}}\right]\right. \\
& \text { for all } x^{\prime} \in\left[x-5 \overline{l_{n}}\left(\beta_{i}\right), x+5 \overline{l_{n}}\left(\beta_{i}\right)\right] \text {, } \\
& \text { and }\left|u_{2, a_{n}^{\alpha_{i}}}\left(s, x^{\prime}\right)-u_{2, a_{n}^{\alpha_{i}}}\left(s, x^{\prime \prime}\right)\right| \leq 2^{-75} a_{n}^{\beta_{i+1}}\left(\left|x^{\prime}-x^{\prime \prime}\right| \vee a_{n}^{\gamma-2 \beta_{i}(1-\gamma)-\varepsilon_{1}}\right) \\
& \text { for all } \left.x^{\prime} \in\left[x-4 \sqrt{a_{n}}, x+4 \sqrt{a_{n}}\right], x^{\prime \prime} \in\left[x^{\prime}-\overline{l_{n}}\left(\beta_{i}\right), x^{\prime}+\overline{l_{n}}\left(\beta_{i}\right)\right]\right\} \text {, }
\end{aligned}
$$


Finally for $0 \leq i \leq L$, set

$$
\tilde{J}_{n, i}=\left\{(s, x): s \geq 0, x \in \tilde{J}_{n, i}(s)\right\} .
$$

Notation. $n_{M}\left(\varepsilon_{1}\right)=\inf \left\{n \in \mathbb{N}: a_{n}^{\varepsilon_{1}} \leq 2^{-M-4}\right\}, n_{0}\left(\varepsilon_{0}, \varepsilon_{1}\right)=\sup \left\{n \in \mathbb{N}: \sqrt{a_{n}}<2^{-a_{n}^{-\varepsilon_{0} \varepsilon_{1} / 4}}\right\}$, where $\sup \emptyset=1$.

The following proposition will be proved in Section 6 .

Proposition $3.3 \tilde{J}_{n, i}(s)$ is a compact set for all $s \geq 0$. There exist stopping times $\left\{U_{M, n} \equiv U_{M, n, K_{0}}: M, n \in \mathbb{N}\right\}$ satisfying $\left(H_{1}\right)$ from Proposition 2.1 such that for $i \in\{0, \ldots, L\}$, $\tilde{J}_{n, i}(s)$ contains $J_{n, i}(s)$ for all $0 \leq s<U_{M, n}$ and

$$
n>n_{M}\left(\varepsilon_{1}\right) \vee n_{0}\left(\varepsilon_{0}, \varepsilon_{1}\right) .
$$

Since $K_{0} \in \mathbb{N}^{\geq K_{1}}$ was arbitrary this proposition implies that there exist stopping times satisfying $\left(H_{1}\right)$ such that the inclusion $\tilde{J}_{n, i}(s) \supset J_{n, i}(s)$ holds up to these stopping times for $n$ sufficiently large. This inclusion means that given a value of the derivative of $u_{1, a_{n}}$ at some point in a small neighborhood $\left[x-\sqrt{a_{n}}, x+\sqrt{a_{n}}\right]$ of $x$ where $|u|$ is small, one can guarantee that the derivative of $u_{1, a_{n}^{\alpha}}$ (for a certain $\alpha$ ) is of the same order at any point in a much larger neighborhood of $x$ (note that $\overline{l_{n}}(\beta) \gg \sqrt{a_{n}}$ for $\left.\beta \leq \frac{1}{2}-6 \varepsilon_{1}\right)$. Moreover we can also control $u_{2, a_{n}^{\alpha}}$ on those long intervals. Our goal now is to show that this implies $\left(H_{2, i}\right)$ for $i=0, \ldots, L$. The next three lemmas provide necessary tools for this.

Throughout the rest of the section we may, and shall, assume that the parameters $M, n \in \mathbb{N}$ satisfy (3.14), although the importance of $n_{0}$ in (3.14) will not be clear until Section 6 .

Lemma 3.4 Assume $i \in\{0, \ldots, L\}, x \in \tilde{J}_{n, i}(s)$ and $\left|x^{\prime}-x\right| \leq 4 \sqrt{a_{n}}$.

(a) If $i>0$, then $\left|u\left(s, x^{\prime \prime}\right)-u\left(s, x^{\prime}\right)\right| \leq 2 a_{n}^{\beta_{i}}\left(\left|x^{\prime \prime}-x^{\prime}\right| \vee a_{n}^{\gamma-2 \beta_{i}(1-\gamma)-\varepsilon_{1}}\right)$ for all $\left|x^{\prime \prime}-x^{\prime}\right| \leq \overline{l_{n}}\left(\beta_{i}\right)$.

(b) If $i<L$, and $a_{n}^{\gamma-2 \beta_{i}(1-\gamma)-\varepsilon_{1}} \leq\left|x^{\prime \prime}-x^{\prime}\right| \leq \overline{l_{n}}\left(\beta_{i}\right)$, then

$$
u\left(s, x^{\prime \prime}\right)-u\left(s, x^{\prime}\right) \begin{cases}\geq 2^{-5} a_{n}^{\beta_{i+1}}\left(x^{\prime \prime}-x^{\prime}\right) & \text { if } x^{\prime \prime} \geq x^{\prime}, \\ \leq 2^{-5} a_{n}^{\beta_{i+1}}\left(x^{\prime \prime}-x^{\prime}\right) & \text { if } x^{\prime \prime} \leq x^{\prime}\end{cases}
$$

Proof. (a) For $n, i, s, x, x^{\prime}, x^{\prime \prime}$ as in (a), we have (since $\left.\beta_{i}+5 \varepsilon_{1}<\frac{1}{2}\right)$

$$
\left|x^{\prime}-x\right| \vee\left|x^{\prime \prime}-x\right| \leq 5 \overline{l_{n}}\left(\beta_{i}\right) \text {. }
$$

We can therefore apply the definition of $\tilde{J}_{n, i}$ and the Mean Value Theorem to conclude that

$$
\begin{aligned}
\left|u\left(s, x^{\prime \prime}\right)-u\left(s, x^{\prime}\right)\right| & \leq\left|u_{1, a_{n}^{\alpha_{i}}}\left(s, x^{\prime \prime}\right)-u_{1, a_{n}^{\alpha_{i}}}\left(s, x^{\prime}\right)\right|+\left|u_{2, a_{n}^{\alpha_{i}}}\left(s, x^{\prime \prime}\right)-u_{2, a_{n}^{\alpha_{i}}}\left(s, x^{\prime}\right)\right| \\
& \leq a_{n}^{\beta_{i}}\left|x^{\prime \prime}-x^{\prime}\right|+2^{-75} a_{n}^{\beta_{i+1}}\left(\left|x^{\prime \prime}-x^{\prime}\right| \vee a_{n}^{\gamma-2 \beta_{i}(1-\gamma)-\varepsilon_{1}}\right) \\
& \leq 2 a_{n}^{\beta_{i}}\left(\left|x^{\prime \prime}-x^{\prime}\right| \vee a_{n}^{\gamma-2 \beta_{i}(1-\gamma)-\varepsilon_{1}}\right) .
\end{aligned}
$$

(b) Consider $n, i, s, x, x^{\prime}, x^{\prime \prime}$ as in (b) with $x^{\prime \prime} \geq x^{\prime}$. We again have (3.15) and can argue as in (a) to see that

$$
\begin{aligned}
u\left(s, x^{\prime \prime}\right)-u\left(s, x^{\prime}\right) & =u_{1, a_{n}^{\alpha_{i}}}\left(s, x^{\prime \prime}\right)-u_{1, a_{n}^{\alpha_{i}}}\left(s, x^{\prime}\right)+u_{2, a_{n}^{\alpha_{i}}}\left(s, x^{\prime \prime}\right)-u_{2, a_{n}^{\alpha_{i}}}\left(s, x^{\prime}\right) \\
& \geq\left(a_{n}^{\beta_{i+1}} / 16\right)\left(x^{\prime \prime}-x^{\prime}\right)-2^{-75} a_{n}^{\beta_{i+1}}\left(x^{\prime \prime}-x^{\prime}\right) \\
& \geq\left(a_{n}^{\beta_{i+1}} / 32\right)\left(x^{\prime \prime}-x^{\prime}\right) .
\end{aligned}
$$


The case $x^{\prime \prime} \leq x^{\prime}$ is similar.

Notation. $l_{n}\left(\beta_{i}\right)=\left(65 a_{n}^{1-\beta_{i+1}}\right) \vee a_{n}^{\gamma-2 \beta_{i}(1-\gamma)-\varepsilon_{1}}, F_{n}(s, x)=\int \Phi^{m_{n+1}}(y-x) u(s, y) d y$.

Lemma 3.5 Assume $i \in\{0, \ldots, L-1\}$ and $(s, x) \in \tilde{J}_{n, i}$.

(a) If $l_{n}\left(\beta_{i}\right) \leq|x-\tilde{x}| \leq \overline{l_{n}}\left(\beta_{i}\right)$, then

$$
F_{n}(s, \tilde{x})-F_{n}(s, x) \begin{cases}\geq 2^{-5} a_{n}^{\beta_{i+1}}(\tilde{x}-x) & \text { if } \tilde{x} \geq x \\ \leq 2^{-5} a_{n}^{\beta_{i+1}}(\tilde{x}-x) & \text { if } \tilde{x} \leq x\end{cases}
$$

(b) $\left[x-\overline{l_{n}}\left(\beta_{i}\right), x-l_{n}\left(\beta_{i}\right)\right] \cup\left[x+l_{n}\left(\beta_{i}\right), x+\overline{l_{n}}\left(\beta_{i}\right)\right] \subset \tilde{J}_{n, i}(s)^{c}$.

Proof. (a) Assume $\tilde{x} \in\left[x+l_{n}\left(\beta_{i}\right), x+\overline{l_{n}}\left(\beta_{i}\right)\right]$. Then

$$
F_{n}(s, \tilde{x})-F_{n}(s, x)=\int_{-\sqrt{a_{n}}}^{\sqrt{a_{n}}} \Phi^{m_{n+1}}(z)(u(s, \tilde{x}+z)-u(s, x+z)) d z .
$$

For $|z| \leq \sqrt{a_{n}}$, let $x^{\prime \prime}=\tilde{x}+z$ and $x^{\prime}=x+z$. Then $\left|x^{\prime}-x\right| \leq \sqrt{a_{n}}$ and

$$
x^{\prime \prime}-x^{\prime}=\tilde{x}-x \in\left[l_{n}\left(\beta_{i}\right), \overline{l_{n}}\left(\beta_{i}\right)\right] .
$$

Therefore Lemma 3.4(b) and (3.16) imply

$$
\begin{aligned}
F_{n}(s, \tilde{x})-F_{n}(s, x) & \geq \int_{-\sqrt{a_{n}}}^{\sqrt{a_{n}}} \Phi^{m_{n+1}}(z) 2^{-5} a_{n}^{\beta_{i+1}}(\tilde{x}-x) d z \\
& =2^{-5} a_{n}^{\beta_{i+1}}(\tilde{x}-x) .
\end{aligned}
$$

The proof for $\tilde{x}<x$ is similar.

(b) If $\tilde{x} \in\left[x-\overline{l_{n}}\left(\beta_{i}\right), x-l_{n}\left(\beta_{i}\right)\right] \cup\left[x+l_{n}\left(\beta_{i}\right), x+\overline{l_{n}}\left(\beta_{i}\right)\right]$, then

$$
\begin{aligned}
\left|F_{n}(s, \tilde{x})\right| & \geq\left|F_{n}(s, \tilde{x})-F_{n}(s, x)\right|-\left|F_{n}(s, x)\right| \\
& \geq 2^{-5} a_{n}^{\beta_{i+1}} l_{n}\left(\beta_{i}\right)-a_{n} \quad\left(\text { by }(\text { a }) \text { and }(s, x) \in \tilde{J}_{n, i}\right) \\
& \geq \frac{33}{32} a_{n} .
\end{aligned}
$$

Therefore $\tilde{x} \notin \tilde{J}_{n, i}(s)$.

To ensure that (b) is not vacuous we obtain some crude lower bounds on the interval given there.

Lemma 3.6 If $i \in\{0, \ldots, L\}$, then

$$
l_{n}\left(\beta_{i}\right)<\sqrt{a_{n}}<\frac{1}{2} \overline{l_{n}}\left(\beta_{i}\right) .
$$




\section{Proof.}

$$
\begin{aligned}
l_{n}\left(\beta_{i}\right) a_{n}^{-1 / 2} & =\left(65 a_{n}^{\frac{1}{2}-\beta_{i+1}}\right) \vee a_{n}^{\gamma-\frac{1}{2}-2 \beta_{i}(1-\gamma)-\varepsilon_{1}} \\
& \leq\left(65 a_{n}^{5 \varepsilon_{1}}\right) \vee a_{n}^{2 \gamma-\frac{3}{2}-\varepsilon_{1}} \quad\left(\text { since } \beta_{i}<1 / 2\right) \\
& <1
\end{aligned}
$$

by (3.10) and because $a_{n}^{5 \varepsilon_{1}}<2^{-20}$ by (3.14). This gives the first inequality. For the second one, use $\beta_{i} \leq \frac{1}{2}-6 \varepsilon_{1}$ and (3.14) to see that

$$
\sqrt{a_{n}} \overline{l_{n}}\left(\beta_{i}\right)^{-1}=a_{n}^{\frac{1}{2}-\beta_{i}-5 \varepsilon_{1}} \leq a_{n}^{\varepsilon_{1}}<1 / 2 .
$$

Let $|A|$ denote the Lebesgue measure of $A \subset \mathbb{R}$.

Lemma 3.7 For all $i \in\{0, \ldots, L-1\}$ and $s \geq 0$,

$$
\left|\tilde{J}_{n, i}(s)\right| \leq 10 K_{0} \overline{l_{n}}\left(\beta_{i}\right)^{-1} l_{n}\left(\beta_{i}\right) .
$$

Proof. Fix $s, i$ as above. Let $\mathcal{I}_{n, i}(x)=\left(x-l_{n}\left(\beta_{i}\right), x+l_{n}\left(\beta_{i}\right)\right) \subset \tilde{\mathcal{I}}_{n, i}(x)=\left(x-\overline{l_{n}}\left(\beta_{i}\right), x+\overline{l_{n}}\left(\beta_{i}\right)\right)$. This inclusion follows from Lemma 3.6. The compactness of $\tilde{J}_{n, i}(s)$ (Proposition 3.3) implies there are $x_{1}, \ldots, x_{Q} \in \tilde{J}_{n, i}(s)$ so that $\tilde{J}_{n, i}(s) \subset \cup_{j=1}^{Q} \mathcal{I}_{n, i}\left(x_{j}\right)$.

Assume that for some $k \neq j,\left|x_{k}-x_{j}\right| \leq \overline{l_{n}}\left(\beta_{i}\right) / 2$. We claim that $\mathcal{I}_{n, i}\left(x_{j}\right) \subset \tilde{\mathcal{I}}_{n, i}\left(x_{k}\right)$. Indeed, if $y \in \mathcal{I}_{n, i}\left(x_{j}\right)$ then

$$
\left|y-x_{k}\right| \leq\left|y-x_{j}\right|+\left|x_{j}-x_{k}\right|<l_{n}\left(\beta_{i}\right)+\overline{l_{n}}\left(\beta_{i}\right) / 2<\overline{l_{n}}\left(\beta_{i}\right),
$$

the last by Lemma 3.6, and the claim is proved. Lemma 3.5(b) implies

$$
\tilde{J}_{n, i}(s) \cap\left(\tilde{\mathcal{I}}_{n, i}\left(x_{k}\right)-\mathcal{I}_{n, i}\left(x_{k}\right)\right)=\emptyset,
$$

and so the above claim gives

$$
\mathcal{I}_{n, i}\left(x_{j}\right) \cap \tilde{J}_{n, i}(s) \subset \tilde{\mathcal{I}}_{n, i}\left(x_{k}\right) \cap \tilde{J}_{n, i}(s)=\mathcal{I}_{n, i}\left(x_{k}\right) \cap \tilde{J}_{n, i}(s) .
$$

Therefore we may omit $\mathcal{I}_{n, i}\left(x_{j}\right)$ and still have a cover of $\tilde{J}_{n, i}(s)$. Doing this sequentially for $x_{1}, \ldots, x_{Q}$, we may therefore assume that

$$
\left|x_{k}-x_{j}\right|>\overline{l_{n}}\left(\beta_{i}\right) / 2 \text { for all } k \neq j .
$$

Since each $x_{j} \in \tilde{J}_{n, i}(s) \subset\left[-K_{0}, K_{0}\right]$, this implies $Q \leq 2 K_{0}\left(\overline{l_{n}}\left(\beta_{i}\right) / 2\right)^{-1}+1$, and therefore

$$
\left|\tilde{J}_{n, i}(s)\right| \leq\left(4 K_{0} \overline{l_{n}}\left(\beta_{i}\right)^{-1}+1\right) 2 l_{n}\left(\beta_{i}\right) \leq 10 K_{0} \overline{l_{n}}\left(\beta_{i}\right)^{-1} l_{n}\left(\beta_{i}\right) .
$$

Proof of $\left(H_{2}\right)$. Fix $M \in \mathbb{N}$. Recall from our discussion after the definition of $J_{n, i}$ sets that it suffices to show that for all $i \in\{0, \ldots, L\}$,

$$
\lim _{n \rightarrow \infty} E\left(I_{i}^{n}\left(t_{0} \wedge U_{M, n}\right)\right)=0 .
$$


We will in fact show that if we strengthen (3.14) to

$$
n>n_{M}\left(\varepsilon_{1}\right) \vee n_{0}\left(\varepsilon_{0}, \varepsilon_{1}\right) \vee \frac{2}{\varepsilon_{1}},
$$

then we have the stronger $\mathbb{L}^{\infty}$ bound

$$
I_{i}^{n}\left(t_{0} \wedge U_{M, n}\right) \leq c_{1}(\Psi) t_{0} K_{0} a_{n}^{\gamma-\frac{3}{4}}
$$

which clearly implies $\left(H_{2, i}\right)$ since $\gamma>\frac{3}{4}$. Proposition 3.3 , Supp $\left(\Phi_{x}^{m_{n+1}}\right) \subset\left[x-\sqrt{a_{n}}, x+\sqrt{a_{n}}\right]$ and $\frac{2}{n}<\varepsilon_{1}$ (by (3.17)) imply

$$
\begin{aligned}
I_{i}^{n}\left(t_{0} \wedge U_{M, n}\right) \leq a_{n}^{-\frac{3}{2}-\varepsilon_{1}} \int_{0}^{t_{0}} \iint & 1\left(s<U_{M, n}\right) 1_{\tilde{J}_{n, i}(s)}(x)|u(s, y)|^{2 \gamma} \\
& \times 1\left(|y-x| \leq \sqrt{a_{n}}\right) \Phi_{x}^{m_{n+1}}(y) \Psi_{s}(x) d y d x d s .
\end{aligned}
$$

Consider first (3.18) for $i=0$. For $x \in \tilde{J}_{n, 0}(s)$ and $|y-x| \leq \sqrt{a_{n}}$, we have $|u(s, y)| \leq 3 a_{n}^{\left(1-\varepsilon_{0}\right) / 2}$ and so from (3.19),

$$
\begin{aligned}
I_{0}^{n}\left(t_{0} \wedge U_{M, n}\right) & \leq a_{n}^{-\frac{3}{2}-\varepsilon_{1}} 3^{2 \gamma} a_{n}^{\gamma\left(1-\varepsilon_{0}\right)}\|\Psi\|_{\infty} \int_{0}^{t_{0}}\left|\tilde{J}_{n, 0}(s)\right| d s \\
& \leq a_{n}^{-\frac{3}{2}-\varepsilon_{1}} 3^{2 \gamma} a_{n}^{\gamma\left(1-\varepsilon_{0}\right)}\|\Psi\|_{\infty} t_{0} 10 K_{0} a_{n}^{-5 \varepsilon_{1}}\left(\left(65 a_{n}^{1-\varepsilon_{0}}\right) \vee a_{n}^{\gamma-\varepsilon_{1}}\right) \quad \text { (by Lemma 3.7) } \\
& \leq c_{1}(\Psi) t_{0} K_{0} a_{n}^{2 \gamma-\frac{3}{2}} a_{n}^{-\gamma \varepsilon_{0}-7 \varepsilon_{1}} \\
& \leq c_{1}(\Psi) t_{0} K_{0} a_{n}^{\gamma-\frac{3}{4}},
\end{aligned}
$$

as required, where (3.10) is used in the last two lines.

Consider now $i \in\{1, \ldots, L\}$. Assume $x \in \tilde{J}_{n, i}(s)$ and $|y-x| \leq \sqrt{a_{n}}$. We have $\left|\left\langle u_{s}, \Phi_{x}^{m_{n+1}}\right\rangle\right| \leq a_{n}$ and

$$
\operatorname{Supp}\left(\Phi_{x}^{m_{n+1}}\right) \subset\left[x-\sqrt{a_{n}}, x+\sqrt{a_{n}}\right] .
$$

Using the continuity of $u(s, \cdot)$, we conclude that

$$
\left|u\left(s, \hat{x}_{n}(s, x)\right)\right| \leq a_{n},
$$

and of course we have $\left|\hat{x}_{n}(s, x)-x\right| \leq \sqrt{a_{n}}$. Therefore

$$
\begin{aligned}
\left|y-\hat{x}_{n}(s, x)\right| & \leq 2 \sqrt{a_{n}} \\
& \leq \overline{l_{n}}\left(\beta_{i}\right) \quad \text { (by Lemma 3.6) }
\end{aligned}
$$

Apply Lemma 3.4(a) with $x^{\prime \prime}=y$ and $x^{\prime}=\hat{x}_{n}(s, x)$, to see that

$$
\begin{aligned}
|u(s, y)| & \leq\left|u\left(s, \hat{x}_{n}(s, x)\right)\right|+2 a_{n}^{\beta_{i}}\left(\left|y-\hat{x}_{n}(s, x)\right| \vee a_{n}^{\gamma-2 \beta_{i}(1-\gamma)-\varepsilon_{1}}\right) \\
& \leq a_{n}+4 a_{n}^{\beta_{i}+\frac{1}{2}} \leq 5 a_{n}^{\beta_{i}+\frac{1}{2}},
\end{aligned}
$$

where (3.20) and Lemma 3.6 are used in the next to last inequality. Use (3.21) in (3.19) and conclude that

$$
I_{i}^{n}\left(t_{0} \wedge U_{M, n}\right) \leq a_{n}^{-\frac{3}{2}-\varepsilon_{1}} 5^{2 \gamma} a_{n}^{2 \gamma\left(\beta_{i}+\frac{1}{2}\right)}\|\Psi\|_{\infty} \int_{0}^{t_{0}}\left|\tilde{J}_{n, i}(s)\right| d s, i=1, \ldots, L .
$$


Assume now $1 \leq i \leq L-1$. Apply Lemma 3.7 to the right-hand side of (3.22) to see that

$$
\begin{aligned}
I_{i}^{n}\left(t_{0} \wedge U_{M, n}\right) & \leq c_{1}(\Psi) t_{0} K_{0} a_{n}^{-\frac{3}{2}-\varepsilon_{1}+2 \gamma\left(\beta_{i}+\frac{1}{2}\right)}\left(a_{n}^{1-\beta_{i+1}} \vee a_{n}^{\gamma-2 \beta_{i}(1-\gamma)-\varepsilon_{1}}\right) a_{n}^{-5 \varepsilon_{1}-\beta_{i}} \\
& =c_{1}(\Psi) t_{0} K_{0}\left[a_{n}^{\rho_{1, i}} \vee a_{n}^{\rho_{2, i}}\right] .
\end{aligned}
$$

A bit of arithmetic shows that

$$
\begin{aligned}
\rho_{1, i} & =\gamma-\frac{1}{2}-2 \beta_{i}(1-\gamma)-\varepsilon_{0}-6 \varepsilon_{1} \\
& >2 \gamma-\frac{3}{2}-\varepsilon_{0}-6 \varepsilon_{1} \quad\left(\text { use } \beta_{i}<1 / 2\right) \\
& >\gamma-\frac{3}{4}
\end{aligned}
$$

where (3.10) is used in the last. We also have

$$
\begin{aligned}
\rho_{2, i} & =-\frac{3}{2}+2 \gamma+\beta_{i}(4 \gamma-3)-7 \varepsilon_{1} \\
& >2 \gamma-\frac{3}{2}-7 \varepsilon_{1}>\gamma-\frac{3}{4},
\end{aligned}
$$

again using (3.10) in the last inequality. Use these bounds on $\rho_{l, i}, l=1,2$ in (3.23) to prove (3.18) for $i \leq i \leq L-1$.

It remains to prove (3.18) for $i=L$. For this, use the trivial bound $\left|\tilde{J}_{n, i}(s)\right| \leq 2 K_{0}$ in (3.22) and obtain

$$
\begin{aligned}
I_{L}^{n}\left(t_{0} \wedge U_{M, n}\right) & \leq a_{n}^{-\frac{3}{2}-\varepsilon_{1}} 5^{2 \gamma} a_{n}^{2 \gamma\left(\beta_{L}+\frac{1}{2}\right)}\|\Psi\|_{\infty} 2 K_{0} t_{0} \\
& \leq c_{1}(\Psi) K_{0} t_{0} a_{n}^{-\frac{3}{2}-\varepsilon_{1}+\gamma+2 \gamma\left(\frac{1}{2}-6 \varepsilon_{1}-\varepsilon_{0}\right)} \\
& \leq c_{1}(\Psi) K_{0} t_{0} a_{n}^{2 \gamma-\frac{3}{2}-15 \varepsilon_{1}} \\
& \leq c_{1}(\Psi) K_{0} t_{0} a_{n}^{\gamma-\frac{3}{4}}
\end{aligned}
$$

yet again using (3.10) in the last. This proves (3.18) in the last case of $i=L$. Having proved (3.18) in all cases, we have finished the proof of $\left(\mathrm{H}_{2}\right)$.

Proposition 2.1 therefore applies and establishes Theorem 1.2 for $b \equiv 0$, except for the proof of Proposition 3.3. This will be the objective of the next three sections.

\section{Some Integral Bounds for Heat Kernels}

If $0<p \leq 1, q \in \mathbb{R}$ and $0 \leq \Delta_{2} \leq \Delta_{1} \leq t$, define

$$
J_{p, q}\left(\Delta_{1}, \Delta_{2}, \Delta\right)=\int_{t-\Delta_{1}}^{t-\Delta_{2}}(t-s)^{q}\left(1 \wedge \frac{\Delta}{t-s}\right)^{p} d s .
$$

These integrals will arise frequently in our modulus of continuity estimates. 
Lemma 4.1 (a) If $q>p-1$, then

$$
J_{p, q}\left(\Delta_{1}, \Delta_{2}, \Delta\right) \leq \frac{2}{q+1-p}\left(\Delta \wedge \Delta_{1}\right)^{p} \Delta_{1}^{q+1-p} .
$$

(b) If $-1<q<p-1$, then

$$
\begin{aligned}
& J_{p, q}\left(\Delta_{1}, \Delta_{2}, \Delta\right) \\
& \quad \leq\left((p-1-q)^{-1}+(q+1)^{-1}\right)\left[\left(\Delta \wedge \Delta_{1}\right)^{q+1} 1\left(\Delta_{2} \leq \Delta\right)+\left(\Delta \wedge \Delta_{1}\right)^{p} \Delta_{2}^{q-p+1} 1\left(\Delta_{2}>\Delta\right)\right] \\
& \quad \leq\left((p-1-q)^{-1}+(q+1)^{-1}\right) \Delta^{p}\left(\Delta \vee \Delta_{2}\right)^{q-p+1} .
\end{aligned}
$$

(c) If $q<-1$, then

$$
J_{p, q}\left(\Delta_{1}, \Delta_{2}, \Delta\right) \leq 2|q+1|^{-1}\left(\Delta \wedge \Delta_{2}\right)^{p} \Delta_{2}^{q+1-p} .
$$

Proof. For all $p, q$ as above,

$$
\begin{aligned}
J_{p, q} & =\int_{\Delta_{2}}^{\Delta_{1}} u^{q}\left(1 \wedge \frac{\Delta}{u}\right)^{p} d u \\
& =1\left(\Delta_{2}<\Delta\right) \int_{\Delta_{2}}^{\Delta \wedge \Delta_{1}} u^{q} d u+1\left(\Delta_{1}>\Delta\right) \int_{\Delta_{2} \vee \Delta}^{\Delta_{1}} \Delta^{p} u^{q-p} d u .
\end{aligned}
$$

(a) From (4.5),

$$
\begin{aligned}
J_{p, q} & \leq 1\left(\Delta_{2}<\Delta\right) \frac{\left(\Delta \wedge \Delta_{1}\right)^{q+1}}{q+1}+1\left(\Delta_{1}>\Delta\right) \Delta^{p} \frac{\Delta_{1}^{q-p+1}}{q-p+1} \\
& \leq\left((q+1)^{-1}+(q-p+1)^{-1}\right)\left(\Delta \wedge \Delta_{1}\right)^{p} \Delta_{1}^{q+1-p}
\end{aligned}
$$

which gives the required bound.

(b) Again (4.5) implies

$$
\begin{aligned}
J_{p, q} & \leq 1\left(\Delta_{2}<\Delta\right) \frac{\left(\Delta \wedge \Delta_{1}\right)^{q+1}}{q+1}+1\left(\Delta_{1}>\Delta\right) \Delta^{p} \frac{\left(\Delta_{2} \vee \Delta\right)^{q+1-p}}{p-1-q} \\
& \leq 1\left(\Delta_{2} \leq \Delta\right)\left(\Delta \wedge \Delta_{1}\right)^{q+1}\left((q+1)^{-1}+(p-1-q)^{-1}\right)+1\left(\Delta_{2}>\Delta\right)\left(\Delta \wedge \Delta_{1}\right)^{p} \Delta_{2}^{q-p+1}
\end{aligned}
$$

which gives the first inequality. The second inequality is elementary.

(c) By (4.5),

$$
\begin{aligned}
J_{p, q} \leq & 1\left(\Delta_{2}<\Delta\right) \frac{\Delta_{2}^{q+1}}{|q+1|}+1\left(\Delta_{1}>\Delta\right) \Delta^{p} \frac{\left(\Delta_{2} \vee \Delta\right)^{q+1-p}}{p-1-q} \\
\leq & \frac{1\left(\Delta_{2}<\Delta\right)}{|q+1|}\left(\Delta_{2} \wedge \Delta\right)^{p} \Delta_{2}^{q+1-p}+1\left(\Delta_{2} \leq \Delta<\Delta_{1}\right) \frac{\Delta^{q+1}}{p-1-q} \\
& +1\left(\Delta<\Delta_{2}\right) \frac{\left(\Delta \wedge \Delta_{2}\right)^{p} \Delta_{2}^{q+1-p}}{p-1-q} \\
\leq & \frac{2}{|q+1|}\left(\Delta \wedge \Delta_{2}\right)^{p} \Delta_{2}^{q+1-p},
\end{aligned}
$$


where we used $\Delta^{q+1} \leq \Delta_{2}^{q+1}=\left(\Delta \wedge \Delta_{2}\right)^{p} \Delta_{2}^{q+1-p}$ if $\Delta_{2} \leq \Delta$, and $|q+1|^{-1} \geq(p-1-q)^{-1}$ in the last line.

We let $p_{t}^{\prime}(x)=\frac{d}{d x} p_{t}(x)$.

\section{Lemma 4.2}

$$
\left|p_{t}^{\prime}(z)\right| \leq \overline{4.2}^{-1 / 2} p_{2 t}(z)
$$

Proof. Trivial.

Lemma 4.3 (a) There is a 4.3 so that for any $s<t \leq t^{\prime}, x, x^{\prime} \in \mathbb{R}$,

$$
\int\left(p_{t^{\prime}-s}\left(y-x^{\prime}\right)-p_{t-s}(y-x)\right)^{2} d y \leq \mathbb{4 . 3}(t-s)^{-1 / 2}\left[1 \wedge \frac{d\left((t, x),\left(t^{\prime}, x^{\prime}\right)\right)^{2}}{t-s}\right] .
$$

(b) For any $R>2$ there is a $\frac{q_{4.3}}{(R)}$ so that for any $0 \leq p, r \leq R, \eta_{0}, \eta_{1} \in(1 / R, 1 / 2), 0 \leq s<t \leq$ $t^{\prime} \leq R, x, x^{\prime} \in \mathbb{R}$

$$
\begin{gathered}
\int e^{r|y-x|}|y-x|^{p}\left(p_{t-s}(y-x)-p_{t^{\prime}-s}\left(y-x^{\prime}\right)\right)^{2} 1\left(|y-x|>\left(t^{\prime}-s\right)^{1 / 2-\eta_{0}} \vee 2\left|x^{\prime}-x\right|\right) d y \\
\leq{ }_{4.3}(R)(t-s)^{-1 / 2} \exp \left\{-\eta_{1}\left(t^{\prime}-s\right)^{-2 \eta_{0}} / 33\right\}\left[1 \wedge \frac{d\left((t, x),\left(t^{\prime}, x^{\prime}\right)\right)^{2}}{t-s}\right]^{1-\left(\eta_{1} / 2\right)} .
\end{gathered}
$$

Proof. (a) Let $f(u)=u^{-1 / 2}$. By Chapman-Kolmogorov, the integral in (4.6) equals

$$
\begin{aligned}
& p_{2\left(t^{\prime}-s\right)}(0)+p_{2(t-s)}(0)-2 p_{t^{\prime}-s+t-s}(0)+2\left(p_{t^{\prime}-s+t-s}(0)-p_{t^{\prime}-s+t-s}\left(x-x^{\prime}\right)\right) \\
& \leq(2 \pi)^{-1 / 2}\left[\left|f\left(2\left(t^{\prime}-s\right)\right)+f(2(t-s))-2 f\left(t^{\prime}-s+t-s\right)\right|\right. \\
& \quad+\left(t^{\prime}-s+t-s\right)^{-1 / 2}\left(1-\exp \left\{\frac{-\left(x-x^{\prime}\right)^{2}}{2\left(t^{\prime}-s+t-s\right)}\right\}\right) \\
& \equiv(2 \pi)^{-1 / 2}\left[T_{1}+T_{2}\right] .
\end{aligned}
$$

Clearly $T_{2}$ is at most $\left(t^{\prime}-s\right)^{-1 / 2}\left[1 \wedge \frac{\left|x-x^{\prime}\right|^{2}}{t^{\prime}-s}\right]$. If $0<u \leq u^{\prime}, 0 \leq f(u)-f\left(u^{\prime}\right) \leq u^{-1 / 2} \wedge\left[u^{-3 / 2}\left|u^{\prime}-u\right|\right]$ (by the Mean Value Theorem) and so

$$
T_{1} \leq\left[\sqrt{2}(t-s)^{-1 / 2} \wedge\left(\frac{2}{(2(t-s))^{3 / 2}}\left|t^{\prime}-t\right|\right)\right]
$$

Use the above bounds on $T_{1}$ and $T_{2}$ in (4.8) to complete the proof of (a).

(b) This proof is very similar to that of Lemma 4.4 (b) below and so is omitted. There are some minor differences leading to the factor of $1 / 33$ (rather than the 1/64 in Lemma 4.4 (b))-e.g., the much simpler analogue of (4.20) below has $p_{u}(w)$ on the right side. 
Lemma 4.4 (a) There is a 4.4 so that for any $s<t \leq t^{\prime}, x, x^{\prime} \in \mathbb{R}$,

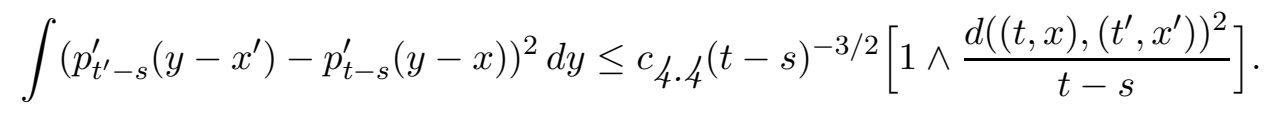

(b) For any $R>2$ there is a $\frac{q_{4.4}(R)}{(R o}$ that for any $0 \leq p, r \leq R, \eta_{0}, \eta_{1} \in(1 / R, 1 / 2), 0 \leq s<t \leq$ $t^{\prime} \leq R, x, x^{\prime} \in \mathbb{R}$

$$
\begin{gathered}
\int e^{r|y-x|}|y-x|^{p}\left(p_{t-s}^{\prime}(y-x)-p_{t^{\prime}-s}^{\prime}\left(y-x^{\prime}\right)\right)^{2} 1\left(|y-x|>\left(t^{\prime}-s\right)^{1 / 2-\eta_{0}} \vee 2\left|x^{\prime}-x\right|\right) d y \\
\leq \mathscr{4 . 4 . 4}(R)(t-s)^{-3 / 2} \exp \left\{-\eta_{1}\left(t^{\prime}-s\right)^{-2 \eta_{0}} / 64\right\}\left[1 \wedge \frac{d\left((t, x),\left(t^{\prime}, x^{\prime}\right)\right)^{2}}{t-s}\right]^{1-\left(\eta_{1} / 2\right)} .
\end{gathered}
$$

Proof. (a) We first claim that

$$
\int p_{t}^{\prime}(w) p_{t}^{\prime}(w-x) d w=\left(\frac{t}{2}-\frac{x^{2}}{4}\right) \frac{p_{2 t}(x)}{t^{2}} .
$$

To see this, first do a bit of algebra to get

$$
p_{t}(w) p_{t}(w-x)=p_{t / 2}(w-(x / 2)) p_{2 t}(x) .
$$

Therefore the left-hand side of (4.12) equals

$$
\begin{aligned}
\int \frac{w(w-x)}{t^{2}} p_{t}(w) p_{t}(w-x) d w & =\int \frac{w(w-x)}{t^{2}} p_{t / 2}(w-(x / 2)) d w p_{2 t}(x) \\
& =\int\left(u^{2}-\frac{x^{2}}{4}\right) p_{t / 2}(u) d u \frac{p_{2 t}(x)}{t^{2}} \quad(u=w-(x / 2)) \\
& =\left(\frac{t}{2}-\frac{x^{2}}{4}\right) \frac{p_{2 t}(x)}{t^{2}}
\end{aligned}
$$

giving the right-hand side of (4.12).

Next we claim that

$$
\int p_{t^{\prime}}^{\prime}(w-x) p_{t}^{\prime}(w-x) d w=\left(t+t^{\prime}\right)^{-1} p_{t^{\prime}+t}(0) .
$$

Some algebra shows that

$$
p_{t^{\prime}}(w) p_{t}(w)=p_{t^{\prime}+t}(0) p_{\frac{t^{\prime} t}{t+t^{\prime}}}(w) .
$$

Therefore the left-hand side of (4.14) equals

$$
\int \frac{w^{2}}{t^{\prime} t} p_{t^{\prime}}(w) p_{t}(w) d w=\int \frac{w^{2}}{t^{\prime} t} p_{\frac{t t^{\prime}}{t+t^{\prime}}}(w) d w p_{t^{\prime}+t}(0)=\left(t+t^{\prime}\right)^{-1} p_{t^{\prime}+t}(0),
$$

and we have (4.14). 
The left-hand side of (4.10) is bounded by

$$
\begin{aligned}
& 2\left[\int\left(p_{t^{\prime}-s}^{\prime}\left(y-x^{\prime}\right)-p_{t^{\prime}-s}^{\prime}(y-x)\right)^{2} d y+\int\left(p_{t^{\prime}-s}^{\prime}(y-x)-p_{t-s}^{\prime}(y-x)\right)^{2} d y\right] \\
& \quad \equiv 2\left(T_{1}+T_{2}\right) .
\end{aligned}
$$

Now expand $T_{1}$ and use (4.12) to see that

$$
\begin{aligned}
T_{1} & =2 \int p_{t^{\prime}-s}^{\prime}(y-x)^{2} d y-2 \int p_{t^{\prime}-s}^{\prime}(w) p_{t^{\prime}-s}^{\prime}\left(w+x-x^{\prime}\right) d w \\
& =\left(t^{\prime}-s\right) \frac{p_{2\left(t^{\prime}-s\right)}(0)}{\left(t^{\prime}-s\right)^{2}}-\left(\left(t^{\prime}-s\right)-\frac{\left(x-x^{\prime}\right)^{2}}{2}\right) \frac{p_{2\left(t^{\prime}-s\right)}\left(x^{\prime}-x\right)}{\left(t^{\prime}-s\right)^{2}} \\
& =\left(t^{\prime}-s\right)^{-1}\left(p_{2\left(t^{\prime}-s\right)}(0)-p_{2\left(t^{\prime}-s\right)}\left(x^{\prime}-x\right)\right)+\frac{\left(x^{\prime}-x\right)^{2}}{2\left(t^{\prime}-s\right)^{2}} p_{2\left(t^{\prime}-s\right)}\left(x^{\prime}-x\right) \\
& \leq\left(t^{\prime}-s\right)^{-3 / 2}\left[1 \wedge \frac{\left(x^{\prime}-x\right)^{2}}{t^{\prime}-s}\right]+\left(\sup _{z}\left(z e^{-z}\right)\left(t^{\prime}-s\right)^{-3 / 2}\right) \wedge\left(\left(x^{\prime}-x\right)^{2}\left(t^{\prime}-s\right)^{-5 / 2}\right) \\
& \leq c_{0}\left(t^{\prime}-s\right)^{-3 / 2}\left[1 \wedge \frac{\left(x^{\prime}-x\right)^{2}}{t^{\prime}-s}\right] .
\end{aligned}
$$

Finally let $g(u)=u^{-3 / 2}$, and expand $T_{2}$ and use (4.14) to conclude

$$
\begin{aligned}
T_{2} & =\left(2\left(t^{\prime}-s\right)\right)^{-1} p_{2\left(t^{\prime}-s\right)}(0)+(2(t-s))^{-1} p_{2(t-s)}(0)-2\left(t-s+t^{\prime}-s\right)^{-1} p_{t-s+t^{\prime}-s}(0) \\
& =(2 \pi)^{-1 / 2}\left[g\left(2\left(t^{\prime}-s\right)\right)+g(2(t-s))-2 g\left(t^{\prime}-s+t-s\right)\right] \\
& \leq(2 \pi)^{-1 / 2} 2\left[(2(t-s))^{-3 / 2} \wedge(2(t-s))^{-5 / 2}\left|t^{\prime}-t\right|\right] .
\end{aligned}
$$

The last inequality follows as in (4.9). Use the above bounds on $T_{1}$ and $T_{2}$ in (4.16) to complete the proof of (a).

(b) Note that $|y-x|>\left(t^{\prime}-s\right)^{1 / 2-\eta_{0}} \vee 2\left|x^{\prime}-x\right|$ implies that

$$
\left|y-x^{\prime}\right| \geq|y-x|-\left|x^{\prime}-x\right| \geq|y-x| / 2 \geq \frac{\left(t^{\prime}-s\right)^{1 / 2-\eta_{0}}}{2}
$$

and in particular from the second inequality,

$$
|y-x| \leq 2\left|y-x^{\prime}\right|
$$

Assume $p, r, \eta_{i}, s, t, t^{\prime}$ as in (b) and let $d=d\left((t, x),\left(t^{\prime}, x^{\prime}\right)\right)$. By Hölder's inequality and then (a), 
the integral on the left-hand side of (4.11) is at most

$$
\begin{aligned}
& {\left[\int\left(p_{t-s}^{\prime}(y-x)-p_{t^{\prime}-s}^{\prime}\left(y-x^{\prime}\right)\right)^{2} d y\right]^{1-\eta_{1} / 2}} \\
& \times\left[\int e^{\frac{2 r}{\eta_{1}}|y-x|}|y-x|^{2 p / \eta_{1}}\left(p_{t-s}^{\prime}(y-x)-p_{t^{\prime}-s}^{\prime}\left(y-x^{\prime}\right)\right)^{2} 1\left(|y-x|>\left(t^{\prime}-s\right)^{1 / 2-\eta_{0}} \vee 2\left|x-x^{\prime}\right|\right) d y\right]^{\eta_{1} / 2} \\
& \leq c_{1}(t-s)^{-\frac{3}{2}+\frac{3 \eta_{1}}{4}}\left[1 \wedge \frac{d^{2}}{t-s}\right]^{1-\frac{\eta_{1}}{2}}\left[\int e^{\frac{2 r}{\eta_{1}}|y-x|}|y-x|^{2 p / \eta_{1}}\left(p_{t-s}^{\prime}(y-x)^{2}+p_{t^{\prime}-s}^{\prime}\left(y-x^{\prime}\right)^{2}\right)\right. \\
& \left.\times 1\left(|y-x|>\left(t^{\prime}-s\right)^{1 / 2-\eta_{0}} \vee 2\left|x-x^{\prime}\right|\right) d y\right]^{\eta_{1} / 2} \\
& \leq c_{2}(R)(t-s)^{-\frac{3}{2}+\frac{3 \eta_{1}}{4}}\left[1 \wedge \frac{d^{2}}{t-s}\right]^{1-\frac{\eta_{1}}{2}}\left[\int e^{\frac{2 r}{\eta_{1}}|w|}|w|^{\frac{2 p}{\eta_{1}}} p_{t-s}^{\prime}(w)^{2} 1\left(|w|>(t-s)^{1 / 2-\eta_{0}}\right) d w\right. \\
& \left.+\int e^{\frac{4 r}{\eta_{1}}|w|}|w|^{\frac{2 p}{\eta_{1}}} p_{t^{\prime}-s}^{\prime}(w)^{2} 1\left(|w|>\left(t^{\prime}-s\right)^{1 / 2-\eta_{0}} / 2\right) d w\right]^{\eta_{1} / 2},
\end{aligned}
$$

where in the last we used (4.17) and (4.18).

If $|w|>\frac{1}{2} u^{1 / 2-\eta_{0}}$, then by Lemma 4.2,

$$
\begin{aligned}
\left.p_{u}^{\prime}(w)^{2} \leq q_{4.2}^{2}\right]^{u^{-1}} p_{2 u}(w)^{2} & \leq q_{4.2}^{2} u^{u^{-3 / 2}} e^{u^{-2 \eta_{0}} / 16} p_{2 u}(w) \\
& \leq c_{3}(R) p_{2 u}(w) .
\end{aligned}
$$

Use this to show that

$$
\begin{aligned}
& {\left[\int e^{\frac{4 r}{\eta_{1}}|w|}|w|^{\frac{2 p}{\eta_{1}}} p_{t^{\prime}-s}^{\prime}(w)^{2} 1\left(|w|>\left(t^{\prime}-s\right)^{1 / 2-\eta_{0}} / 2\right) d w\right]^{\eta_{1} / 2}} \\
& \leq c_{4}(R)\left[\int e^{\frac{4 r}{\eta_{1}}|w|}|w|^{\frac{2 p}{\eta_{1}}} p_{2\left(t^{\prime}-s\right)}(w) 1\left(|w|>\left(t^{\prime}-s\right)^{1 / 2-\eta_{0}} / 2\right) d w\right]^{\eta_{1} / 2} \\
& \leq c_{5}(R) E\left(e^{\frac{8 r}{\eta_{1}} \sqrt{2\left(t^{\prime}-s\right)}\left|B_{1}\right|}\left|B_{1}\right|^{\frac{4 p}{\eta_{1}}}\right)^{\eta_{1} / 4} P\left(\left|B_{1}\right|>\frac{1}{2 \sqrt{2}}\left(t^{\prime}-s\right)^{-\eta_{0}}\right)^{\eta_{1} / 4} \quad \text { (by Hölder) } \\
& \leq c_{6}(R) \exp \left\{-\eta_{1}\left(t^{\prime}-s\right)^{-2 \eta_{0}} / 64\right\} .
\end{aligned}
$$

Use the same bound with $t$ in place of $t^{\prime}$ to see that the right-hand side of (4.19), and hence of (4.11), is at most

$$
c_{7}(R)(t-s)^{3 \eta_{1} / 4}(t-s)^{-3 / 2}\left[1 \wedge \frac{d^{2}}{t-s}\right]^{1-\frac{\eta_{1}}{2}} \exp \left\{-\left(t^{\prime}-s\right)^{-2 \eta_{0}} \eta_{1} / 64\right\} .
$$

The result follows because $(t-s)^{3 \eta_{1} / 4} \leq R$.

\section{Local Bounds on the Difference of Two Solutions}

This section is devoted to establishing the local bounds on the difference of two solutions to (1.5). These bounds are crucial for the construction of the stopping times in Proposition 3.3, which is then carried out in Section 6. We continue to assume throughout this Section that $b \equiv 0$. Recall 
that $X^{1}, X^{2}$ are two solutions as in Section 2, $u=X^{1}-X^{2}$, and we assume the hypotheses of Theorem 1.2 as well as (2.29).

We refine the earlier set $Z(N, K)$ and define, for $K, N, n \in \mathbb{N}$ and $\beta \in(0,1 / 2]$,

$Z(N, n, K, \beta)(\omega)=\left\{(t, x) \in\left[0, T_{K}\right] \times[-K, K]:\right.$ there is a $\left(\hat{t}_{0}, \hat{x}_{0}\right) \in\left[0, T_{K}\right] \times \mathbb{R}$ such that $d\left(\left(\hat{t}_{0}, \hat{x}_{0}\right),(t, x)\right) \leq 2^{-N},\left|u\left(\hat{t}_{0}, \hat{x}_{0}\right)\right| \leq a_{n} \wedge\left(\sqrt{a_{n}} 2^{-N}\right)$, and $\left.\left|u_{1, a_{n}}^{\prime}\left(\hat{t}_{0}, \hat{x}_{0}\right)\right| \leq a_{n}^{\beta}\right\}$,

and for $\beta=0$ define $Z(N, n, K, 0)(\omega)=Z(N, n, K)(\omega)$ as above, but with the condition on $\left|u_{1, a_{n}}^{\prime}\left(\hat{t}_{0}, \hat{x}_{0}\right)\right|$ omitted.

Recalling $\gamma<1$, we let

$$
\gamma_{m}=\frac{(\gamma-1 / 2)\left(1-\gamma^{m}\right)}{1-\gamma}+1, \quad \tilde{\gamma}_{m}=\gamma_{m} \wedge 2
$$

so that we have the recursion relation

$$
\gamma_{m+1}=\gamma \gamma_{m}+1 / 2, \quad \gamma_{0}=1 .
$$

Clearly $\gamma_{m}$ increases to $\gamma_{\infty}=(\gamma-1 / 2)(1-\gamma)^{-1}+1=(2(1-\gamma))^{-1}>2$ and so we may define a finite natural number, $\bar{m}>1$, by

$$
\bar{m}=\min \left\{m: \gamma_{m+1}>2\right\}=\min \left\{m: \gamma \gamma_{m}>3 / 2\right\} .
$$

Definition. A collection of $[0, \infty]$-valued random variables, $\{N(\alpha): \alpha \in A\}$, is stochastically bounded uniformly in $\alpha$ iff

$$
\lim _{M \rightarrow \infty} \sup _{\alpha \in A} P(N(\alpha) \geq M)=0 .
$$

Finally we introduce the condition whose proof will be the goal of this section. Recall that $K_{1}$ is as in (2.36).

Property $\left(P_{m}\right)$. For $m \in \mathbb{Z}_{+}$we will let $\left(P_{m}\right)$ denote the following property:

For any $n \in \mathbb{N}, \xi, \varepsilon_{0} \in(0,1), K \in \mathbb{N}^{\geq K_{1}}$ and $\beta \in[0,1 / 2]$, there is an $N_{1}(\omega)=N_{1}\left(m, n, \xi, \varepsilon_{0}, K, \beta\right)$ in $\mathbb{N}$ a.s. such that for all $N \geq N_{1}$, if $(t, x) \in Z(N, n, K, \beta), t^{\prime} \leq T_{K}$, and $d\left((t, x),\left(t^{\prime}, x^{\prime}\right)\right) \leq 2^{-N}$, then $\left|u\left(t^{\prime}, x^{\prime}\right)\right| \leq a_{n}^{-\varepsilon_{0}} 2^{-N \xi}\left[\left(\sqrt{a_{n}} \vee 2^{-N}\right)^{\tilde{\gamma}_{m}-1}+a_{n}^{\beta} 1(m>0)\right]$.

Moreover $N_{1}$ is stochastically bounded uniformly in $(n, \beta)$.

Here is the main result of this section:

Proposition 5.1 For any $m \leq \bar{m}+1,\left(P_{m}\right)$ holds. 
Proof. $\quad\left(P_{0}\right)$ is an easy consequence of Theorem 2.3, as we now show-and we may even take $\varepsilon_{0}=0$. Let $\xi \in(0,1)$ and apply Theorem 2.3 with $\xi^{\prime}=(\xi+1) / 2$ in place of $\xi$. If $(t, x) \in Z(N, K)(\supset$ $Z(N, n, K, \beta))$ and $\left(\hat{t}_{0}, \hat{x}_{0}\right)$ is as in the definition of $Z(N, K)$, then $\left(\hat{t}_{0}, \hat{x}_{0}\right) \in Z(N, K+1)$ (we need $K+1$ since $\left.\left|\hat{x}_{0}\right| \leq K+1\right)$. Theorem 2.3 implies that if $N \geq N_{0}\left(\xi^{\prime}, K+1\right) \vee 4(1-\xi)^{-1} \equiv N_{1}(0, \xi, K)$ (it doesn't depend on $(n, \beta)$ and there is no $\left.\varepsilon_{0}\right)$, then

$$
|u(t, x)| \leq 2^{-N \xi^{\prime}}+\left|u\left(\hat{t}_{0}, \hat{x}_{0}\right)\right| \leq 2^{1-N \xi^{\prime}} .
$$

If $\left(t^{\prime}, x^{\prime}\right)$ is as in $\left(P_{0}\right)$, the above and Theorem 2.3 imply

$$
\left|u\left(t^{\prime}, x^{\prime}\right)\right| \leq|u(t, x)|+\left|u\left(t^{\prime}, x^{\prime}\right)-u(t, x)\right| \leq 2^{1-N \xi^{\prime}}+2^{-N \xi^{\prime}} \leq 2^{2-N \xi^{\prime}} \leq 2^{-N \xi},
$$

where the last inequality holds because $N \geq 4(1-\xi)^{-1}$. $\left(P_{0}\right)$ follows.

The induction step will require some additional continuity results which also will be used directly in the next section. We start by noting that $\left(P_{m}\right)$ easily gives some global bounds on $|u|$.

Lemma 5.2 Let $0 \leq m \leq \bar{m}+1$ and assume $\left(P_{m}\right)$. For any $n, \xi, \varepsilon_{0}, K$ and $\beta$ as in $\left(P_{m}\right)$, if $\bar{d}_{N}=2^{-N} \vee d((s, y),(t, x))$ and $\sqrt{C_{5.2}(\omega)}=\left(4 a_{n}^{-\varepsilon_{0}}+2^{2 N_{1}(\omega)} 2 K e^{K}\right)$, then for any $N \in \mathbb{N}$,

$$
\text { on }\left\{\omega: N \geq N_{1}\left(m, n, \xi, \varepsilon_{0}, K, \beta\right),(t, x) \in Z(N, n, K, \beta)\right\} \text {, }
$$

we have

$$
\begin{aligned}
|u(s, y)| \leq & \sqrt{\overline{C F .2}^{|y-x|}} \bar{d}_{N}^{\xi} \\
& \times\left[\left(\sqrt{a_{n}} \vee \bar{d}_{N}\right)^{\tilde{\gamma}_{m-1}}+1(m>0) a_{n}^{\beta}\right] \text { for all } s \leq T_{K} \text { and } y \in \mathbb{R} .
\end{aligned}
$$

Proof. Assume $N, \omega, t, x$ are as in (5.4).

Case 1. $d \equiv d((s, y),(t, x)) \leq 2^{-N_{1}}$.

If $d>2^{-N}$ choose $N_{1} \leq N^{\prime}<N$ so that $2^{-N^{\prime}-1}<d \leq 2^{-N^{\prime}}$, and if $d \leq 2^{-N}$ set $N^{\prime}=N$. Then $(t, x) \in Z\left(N^{\prime}, n, K, \beta\right), \bar{d} \leq 2^{-N^{\prime}} \leq 2^{-N} \vee 2 d \leq 2 \bar{d}_{N}$ and so by $\left(P_{m}\right)$ for $s \leq T_{K}$,

$$
\begin{aligned}
|u(s, y)| & \leq a_{n}^{-\varepsilon_{0}} 2^{-N^{\prime} \xi}\left[\left(\sqrt{a_{n}} \vee 2^{-N^{\prime}}\right)^{\tilde{\gamma}_{m}-1}+1(m>0) a_{n}^{\beta}\right] \\
& \leq 4 a_{n}^{-\varepsilon_{0}}\left(\bar{d}_{N}\right)^{\xi}\left[\left(\sqrt{a_{n}} \vee \bar{d}_{N}\right)^{\tilde{\gamma}_{m}-1}+1(m>0) a_{n}^{\beta}\right] .
\end{aligned}
$$

Case 2. $d>2^{-N_{1}}$.

As $K \geq K_{1}$, for $s \leq T_{K}$,

$$
\begin{aligned}
|u(s, y)| \leq 2 K e^{|y|} & \leq 2 K e^{|y|}\left(d 2^{N_{1}}\right)^{\xi+\tilde{\gamma}_{m}-1} \\
& \leq 2 K e^{K} e^{|y-x|} 2^{2 N_{1}}\left(\bar{d}_{N}\right)^{\xi+\tilde{\gamma}_{m}-1}
\end{aligned}
$$

The Lemma follows from the above two bounds.

Remark 5.3 If $m=0$ we may set $\varepsilon_{0}=0$ in the above and $N_{1}$ will not depend on $\left(n, \varepsilon_{0}, \beta\right)$ by the above proof of $\left(P_{0}\right)$. 
To carry out the induction we first use $\left(P_{m}\right)$ to obtain a local modulus of continuity for $F_{\delta}$. From Lemma 3.1, we have for $s \leq t \leq t^{\prime}$ and $s^{\prime} \leq t^{\prime}$

$$
\begin{aligned}
\left|F_{\delta}(s, t, x)-F_{\delta}\left(s^{\prime}, t^{\prime}, x^{\prime}\right)\right| \leq & \left|F_{\delta}\left(s, t^{\prime}, x^{\prime}\right)-F_{\delta}\left(s^{\prime}, t^{\prime}, x^{\prime}\right)\right|+\left|F_{\delta}\left(s, t^{\prime}, x^{\prime}\right)-F_{\delta}(s, t, x)\right| \\
= & \left|\int_{(s-\delta)^{+}}^{\left(s^{\prime}-\delta\right)^{+}} \int p_{t^{\prime}-r}^{\prime}\left(y-x^{\prime}\right) D(r, y) W(d r, d y)\right| \\
& +\left|\int_{0}^{(s-\delta)^{+}}\left(p_{t^{\prime}-r}^{\prime}\left(y-x^{\prime}\right)-p_{t-r}^{\prime}(y-x)\right) D(r, y) W(d r, d y)\right| .
\end{aligned}
$$

This decomposition and (3.2) suggest we introduce the following square functions for $\eta_{0} \in(0,1 / 2)$ and $\delta \in(0,1]$, and $s \leq t \leq t^{\prime}, s^{\prime} \leq t^{\prime}$ :

$$
\begin{aligned}
& Q_{T, \delta}\left(s, s^{\prime}, t^{\prime}, x^{\prime}\right)=\int_{\left(s \wedge s^{\prime}-\delta\right)^{+}}^{\left(s \vee s^{\prime}-\delta\right)^{+}} \int p_{t^{\prime}-r}^{\prime}\left(y-x^{\prime}\right)^{2} e^{2 R_{1}|y|}|u(r, y)|^{2 \gamma} d y d r \\
& Q_{S, 1, \delta, \eta_{0}}\left(s, t, x, t^{\prime}, x^{\prime}\right)=\int_{0}^{(s-\delta)^{+}} \int 1\left(|y-x|>\left(t^{\prime}-r\right)^{1 / 2-\eta_{0}} \vee 2\left|x-x^{\prime}\right|\right) \\
& \times\left(p_{t^{\prime}-r}^{\prime}\left(y-x^{\prime}\right)-p_{t-r}^{\prime}(y-x)\right)^{2} e^{2 R_{1}|y|}|u(r, y)|^{2 \gamma} d y d r \\
& Q_{S, 2, \delta, \eta_{0}}\left(s, t, x, t^{\prime}, x^{\prime}\right)=\int_{0}^{(s-\delta)^{+}} \int 1\left(|y-x| \leq\left(t^{\prime}-r\right)^{1 / 2-\eta_{0}} \vee 2\left|x-x^{\prime}\right|\right) \\
& \times\left(p_{t^{\prime}-r}^{\prime}\left(y-x^{\prime}\right)-p_{t-r}^{\prime}(y-x)\right)^{2} e^{2 R_{1}|y|}|u(r, y)|^{2 \gamma} d y d r .
\end{aligned}
$$

Lemma 5.4 For all $K \in \mathbb{N}^{\geq K_{1}}, R>2$ there is a $45.4(K, R)$ and an $N_{5.4}=N_{2}(K, \omega) \in \mathbb{N}$ a.s. so that for all $\eta_{0}, \eta_{1} \in(1 / R, 1 / 2), \delta \in(0,1], \beta \in[0,1 / 2]$ and $N, n \in \mathbb{N}$, for any $(t, x) \in \mathbb{R}_{+} \times \mathbb{R}$, on

$$
\{\omega:(t, x) \in Z(N, n, K, \beta), N \geq N[5.4\},
$$

$Q_{S, 1, \delta, \eta_{0}}\left(s, t, x, t^{\prime}, x^{\prime}\right) \leq \Phi 5.4^{4 \Lambda}\left[\sqrt[5.4]{(\omega)}\left[d^{2-\eta_{1}}+(d \wedge \sqrt{\delta})^{2-\eta_{1}} \delta^{-3 / 2}(d \wedge 1)^{4 \gamma}\right]\right.$ for all $s \leq t \leq t^{\prime}$ and $x^{\prime} \in \mathbb{R}$.

Here $d=d\left(\left(t^{\prime}, x^{\prime}\right),(t, x)\right)$.

Proof We let $N_{5.4}(K, \omega)=N_{1}(0,3 / 4, K)$, that is we recall from Remark 5.3 that for $m=0, N_{1}$ depends only on $\xi$ and $K$ and we take $\xi=3 / 4$. We may assume $\delta<s$ as the left-hand side is 0 
otherwise. Then for $\omega$ as in (5.7) and $s \leq t \leq t^{\prime}$, Lemma 5.2 with $m=0$ implies

$$
\begin{aligned}
& Q_{S, 1, \delta, \eta_{0}}\left(s, t, x, t^{\prime}, x^{\prime}\right) \\
& \leq C_{5.2}(\omega) \int_{0}^{(s-\delta)^{+}} \int 1\left(|y-x|>\left(t^{\prime}-r\right)^{1 / 2-\eta_{0}} \vee 2\left|x-x^{\prime}\right|\right)\left(p_{t^{\prime}-r}^{\prime}\left(y-x^{\prime}\right)-p_{t-r}^{\prime}(y-x)\right)^{2} \\
& \times e^{2 R_{1}|y|} e^{2|y-x|}\left(2^{-N} \vee(\sqrt{t-r}+|y-x|)\right)^{\gamma 3 / 2} d y d s \\
& \leq G_{5.2}(\omega) \int_{0}^{(s-\delta)^{+}} \int 1\left(|y-x|>\left(t^{\prime}-r\right)^{1 / 2-\eta_{0}} \vee 2\left|x-x^{\prime}\right|\right)\left(p_{t^{\prime}-r}^{\prime}\left(y-x^{\prime}\right)-p_{t-r}^{\prime}(y-x)\right)^{2} \\
& \times e^{2 R_{1} K} e^{2\left(R_{1}+1\right)|y-x|}\left[2 K^{\gamma 3 / 4}+2|y-x|^{\gamma 3 / 2}\right] d y d s \\
& \leq C_{\overline{5.2}}(\omega) c_{0}(K, R) \int_{0}^{s-\delta}(t-r)^{-3 / 2} \exp \left\{\frac{-\eta_{1}\left(t^{\prime}-r\right)^{-2 \eta_{0}}}{64}\right\}\left[1 \wedge \frac{d^{2}}{t-r}\right]^{1-\eta_{1} / 2} d r .
\end{aligned}
$$

In the last line we have used Lemma 4.4(b). Use the trivial bound (recall $r \leq s \leq t \leq t^{\prime}$ )

$$
\exp \left\{\frac{-\eta_{1}\left(t^{\prime}-r\right)^{-2 \eta_{0}}}{64}\right\} \leq \exp \left\{\frac{-\eta_{1}\left(t^{\prime}-t\right)^{-2 \eta_{0}}}{128}\right\}+\exp \left\{\frac{-\eta_{1}(t-r)^{-2 \eta_{0}}}{128}\right\}
$$

and then Lemma 4.1 in the above, to bound $Q_{S, 1, \delta, \eta_{0}}\left(s, t, x, t^{\prime}, x^{\prime}\right)$ by

$$
\begin{aligned}
& C_{5.2}(\omega) c_{0}(K, R)\left[\int_{0}^{s-\delta}(t-r)^{-3 / 2}\left[1 \wedge \frac{d^{2}}{t-r}\right]^{1-\eta_{1} / 2} d r \exp \left\{\frac{-\eta_{1}\left(t^{\prime}-t\right)^{-2 \eta_{0}}}{128}\right\}\right. \\
& \left.+\int_{0}^{s-\delta}(t-r)^{-3 / 2}\left(1 \wedge \frac{d^{2}}{t-r}\right)^{1-\eta_{1} / 2} \exp \left\{\frac{-\eta_{1}(t-r)^{-2 \eta_{0}}}{128}\right\} d r\right] \\
& \leq C_{5.2}(\omega) c_{1}(K, R)\left[\left(d^{2} \wedge \delta\right)^{1-\eta_{1} / 2} \delta^{-3 / 2} \exp \left\{\frac{-\eta_{1}\left(t^{\prime}-t\right)^{-2 \eta_{0}}}{128}\right\}+C_{1}(R) \int_{0}^{s-\delta}\left(1 \wedge \frac{d^{2}}{t-r}\right)^{1-\eta / 2} d r\right] \\
& \leq C_{5.2}(\omega) c_{2}(K, R)\left[(d \wedge \sqrt{\delta})^{2-\eta_{1}} \delta^{-3 / 2}(d \wedge 1)^{4 \gamma}+d^{2-\eta_{1}}\right] .
\end{aligned}
$$

Now since we may set $\varepsilon_{0}=0$ in the formula for $C_{5.2}$ by Remark 5.3 , the result follows.

Lemma 5.5 Let $0 \leq m \leq \bar{m}+1$ and assume $\left(P_{m}\right)$. For any $K \in \mathbb{N}^{\geq K_{1}}, R>2, n \in \mathbb{N}, \varepsilon_{0} \in(0,1)$, and $\beta \in[0,1 / 2]$ there is a $\overline{5.5}(K, R)$ and $N_{5.5}=N_{5.5}\left(m, n, R, \varepsilon_{0}, K, \beta\right)(\omega) \in \mathbb{N}$ a.s. such that for any $\eta_{1} \in\left(R^{-1}, 1 / 2\right), \eta_{0} \in\left(0, \eta_{1} / 32\right), \delta \in\left[a_{n}, 1\right], N \in \mathbb{N}$, and $(t, x) \in \mathbb{R}_{+} \times \mathbb{R}$, on

$$
\begin{gathered}
\{\omega:(t, x) \in Z(N, n, K, \beta), N \geq N[5.5] \\
Q_{S, 2, \delta, \eta_{0}}\left(s, t, x, t^{\prime}, x^{\prime}\right) \\
\leq \text { 5.5.5 }(K, R)\left[a_{n}^{-2 \varepsilon_{0}}+2^{4 N}[\overline{5.5}]\left[d^{2-\eta_{1}}\left[\bar{\delta}_{N}^{\left(\gamma \gamma_{m}-3 / 2\right) \wedge 0}+a_{n}^{2 \beta \gamma} \bar{\delta}_{N}^{\left(\gamma-\frac{3}{2}\right) \wedge 0}\right]\right.\right. \\
\left.+(d \wedge \sqrt{\delta})^{2-\eta_{1}} \delta^{-3 / 2}\left[\bar{d}_{N}^{2 \gamma \tilde{\gamma}_{m}}+a_{n}^{2 \beta \gamma} \bar{d}_{N}^{2 \gamma}\right]\right] \\
\text { for all } s \leq t \leq t^{\prime} \leq K,\left|x^{\prime}\right| \leq K+1 .
\end{gathered}
$$

Here $d=d\left((t, x),\left(t^{\prime}, x^{\prime}\right)\right), \bar{d}_{N}=d \vee 2^{-N}$ and $\bar{\delta}_{N}=\delta \vee \bar{d}_{N}^{2}$. Moreover ${ }_{5.5}$ is stochastically bounded uniformly in $(n, \beta)$. 
Proof. Let $\xi=1-(8 R)^{-1} \in(15 / 16,1)$ and define $N_{5.5}=N_{1}\left(m, n, \xi, \varepsilon_{0}, K, \beta\right)$ so that the last statement is immediate from $\left(P_{m}\right)$. We may assume $s \geq \delta$, or the left-hand side is 0 . As $\delta \geq a_{n}$, when we use Lemma 5.2 to bound $u(r, y)$ in the integral defining $Q_{S, 2, \delta, \eta_{0}}$, we have $d((r, y),(t, x)) \geq$ $\sqrt{a_{n}}$ and so we may drop the max with $\sqrt{a_{n}}$. So for $\omega$ as in (5.9), $s \leq t \leq t^{\prime}$ and $\left|x^{\prime}\right| \leq K+1$, Lemma 5.2 implies that

$$
\begin{aligned}
& Q_{S, 2, \delta, \eta_{0}}\left(s, t, x, t^{\prime}, x^{\prime}\right) \\
& \leq q_{5.2} \int_{0}^{s-\delta} \int\left(p_{t^{\prime}-r}^{\prime}\left(y-x^{\prime}\right)-p_{t-r}^{\prime}(y-x)\right)^{2} e^{2 R_{1} K} e^{2\left(R_{1}+1\right) 2(2 K+1)} d y \\
& \times\left[2^{-N} \vee\left((t-r)^{1 / 2}+\left(t^{\prime}-r\right)^{1 / 2-\eta_{0}} \vee\left(2\left|x-x^{\prime}\right|\right)\right)\right]^{2 \gamma \xi} \\
& \times\left\{\left[2^{-N} \vee\left((t-r)^{1 / 2}+\left(t^{\prime}-r\right)^{1 / 2-\eta_{0}} \vee\left(2\left|x-x^{\prime}\right|\right)\right)\right]^{\tilde{\gamma}_{m}-1}+a_{n}^{\beta}\right\}^{2 \gamma} d r
\end{aligned}
$$

Let $\gamma^{\prime}=\gamma\left(1-2 \eta_{0}\right)$. Recall that $t \leq t^{\prime} \leq K,|x| \leq K$ and $\left|x^{\prime}\right| \leq K+1$, so that $\sqrt{t-r} \leq$ $K^{\eta_{0}}\left(t^{\prime}-r\right)^{1 / 2-\eta_{0}}$ and $\left|x-x^{\prime}\right| \leq(2 K+1)\left|x-x^{\prime}\right|^{1-2 \eta_{0}}$. Use this and Lemma 4.4(a) to see that the above is at most

$$
\begin{aligned}
& c_{1}(K) C_{5.2} \int_{0}^{s-\delta}(t-r)^{-3 / 2}\left(1 \wedge \frac{d^{2}}{t-r}\right)\left(2^{-2 N \gamma} \vee\left(t^{\prime}-r\right)^{\gamma^{\prime}} \vee\left|x-x^{\prime}\right|^{2 \gamma^{\prime}}\right)^{\xi} \\
& \times\left[2^{-2 N \gamma\left(\tilde{\gamma}_{m}-1\right)} \vee\left(t^{\prime}-r\right)^{\gamma^{\prime}\left(\tilde{\gamma}_{m}-1\right)} \vee\left|x^{\prime}-x\right|^{2 \gamma^{\prime}\left(\tilde{\gamma}_{m}-1\right)}+a_{n}^{2 \beta \gamma}\right] d r
\end{aligned}
$$

Note that

$$
2^{-2 N \gamma} \vee\left(t^{\prime}-r\right)^{\gamma^{\prime}} \vee\left|x^{\prime}-x\right|^{2 \gamma^{\prime}} \leq 2^{-2 N \gamma^{\prime}} \vee d^{2 \gamma^{\prime}}+(t-r)^{\gamma^{\prime}} \leq 2\left[\vec{d}_{N}^{2 \gamma^{\prime}} \vee(t-r)^{\gamma^{\prime}}\right]
$$

Use this to bound the summands in (5.10) and conclude that

$$
\begin{aligned}
& Q_{S, 2, \delta, \eta_{0}}\left(s, t, x, t^{\prime}, x^{\prime}\right) \\
& \leq c_{2}(K) q_{5.2} \int_{0}^{s-\delta}(t-r)^{-3 / 2}\left(1 \wedge \frac{d^{2}}{t-r}\right)\left[\bar{d}_{N}^{2} \vee(t-r)\right]^{\gamma^{\prime} \xi} \\
& \times\left[\left[\bar{d}_{N}^{2} \vee(t-r)\right]^{\gamma^{\prime}\left(\tilde{\gamma}_{m}-1\right)}+a_{n}^{2 \beta \gamma}\right] d r \\
& \leq c_{2}(K) C_{5.2}\left\{\int_{0}^{t-\bar{\delta}_{N}}(t-r)^{\gamma^{\prime} \xi-3 / 2}\left(1 \wedge \frac{d^{2}}{t-r}\right)\left[(t-r)^{\gamma^{\prime}\left(\tilde{\gamma}_{m}-1\right)}+a_{n}^{2 \beta \gamma}\right] d r\right. \\
& \left.+\int_{0}^{t-\delta}(t-r)^{-3 / 2}\left(1 \wedge \frac{d^{2}}{t-r}\right) d r \vec{d}_{N}^{2 \gamma^{\prime} \xi}\left[\vec{d}_{N}^{2 \gamma^{\prime}\left(\tilde{\gamma}_{m}-1\right)}+a_{n}^{2 \beta \gamma}\right]\right\} \\
& \equiv c_{2}(K) C_{5.2}\left\{I_{1}+I_{2}\right\} \text {. }
\end{aligned}
$$

Apply Lemma 4.1(c) to see that

$$
I_{2} \leq(d \wedge \sqrt{\delta})^{2} \delta^{-3 / 2} \vec{d}_{N}^{2 \gamma^{\prime} \xi}\left[\vec{d}_{N}^{2 \gamma^{\prime}\left(\tilde{\gamma}_{m}-1\right)}+a_{n}^{2 \beta \gamma}\right]
$$


In the integral defining $I_{1}$ we may drop the minimum with 1 and, adding a $\log \left(1 / \bar{\delta}_{N}\right)$ factor just in case the exponent on $u$ is -1 , we arrive at

$$
\begin{aligned}
I_{1} & \leq d^{2}\left[\int_{\bar{\delta}_{N}}^{t} u^{\gamma^{\prime}\left(\tilde{\gamma}_{m}+\xi-1\right)-5 / 2} d u+a_{n}^{2 \beta \gamma} \int_{\bar{\delta}_{N}}^{t} u^{\gamma^{\prime} \xi-5 / 2} d u\right] \\
& \leq c_{3}(K) d^{2} \log \left(1 / \bar{\delta}_{N}\right)\left[\bar{\delta}_{N}^{\left[\gamma^{\prime}\left(\tilde{\gamma}_{m}+\xi-1\right)-3 / 2\right] \wedge 0}+a_{n}^{2 \beta \gamma} \bar{\delta}_{N}^{\left[\gamma^{\prime} \xi-3 / 2\right] \wedge 0}\right]
\end{aligned}
$$

The $\log 1 / \bar{\delta}_{N}$ is bounded by $c(R) d^{-\eta_{1} / 2}$. A bit of arithmetic shows that our conditions $\eta_{0} \leq \eta_{1} / 32$, $1-\xi=1 / 8 R$ and $\eta_{1}>1 / R$ allow us to shift $\xi$ to 1 and $\gamma^{\prime}$ to $\gamma$ in the exponents on the right-hand sides of (5.13) and (5.14) at the cost of multiplying by $d^{-\eta_{1} / 2}$. So using this, (5.13) and (5.14) in (5.12), we get

$$
\begin{aligned}
& Q_{S, 2, \delta, \eta_{0}}\left(s, t, x, t^{\prime}, x^{\prime}\right) \\
& \leq c_{4}(K, R) q_{5.2}\left\{d^{2-\eta_{1}}\left[\bar{\delta}_{N}^{\left[\gamma \tilde{\gamma}_{m}-3 / 2\right] \wedge 0}+a_{n}^{2 \beta \gamma} \bar{\delta}_{N}^{[\gamma-3 / 2] \wedge 0}\right]\right. \\
& \left.+(d \wedge \sqrt{\delta})^{2-\eta_{1}} \delta^{-3 / 2}\left[\bar{d}_{N}^{2 \gamma \tilde{\gamma}_{m}}+a_{n}^{2 \beta \gamma} \bar{d}_{N}^{2 \gamma}\right]\right\} .
\end{aligned}
$$

The result follows from the definition of $C_{5.2}$ and the identity

$$
\left[\gamma\left(\gamma_{m} \wedge 2\right)-3 / 2\right] \wedge 0=\left(\gamma \gamma_{m}-3 / 2\right) \wedge 0
$$

(use $\gamma>3 / 4$ here).

Lemma 5.6 Let $0 \leq m \leq \bar{m}+1$ and assume $\left(P_{m}\right)$. For any $K \in \mathbb{N} \geq K_{1}, R>2, n \in \mathbb{N}, \varepsilon_{0} \in(0,1)$, and $\beta \in[0,1 / 2]$ there is a $95.6(K)$ and $N_{5.6}=N_{5.6}\left(m, n, R, \varepsilon_{0}, K, \beta\right)(\omega) \in \mathbb{N}$ a.s. such that for any $\eta_{1} \in\left(R^{-1}, 1 / 2\right), \delta \in\left[a_{n}, 1\right], N \in \mathbb{N}$, and $(t, x) \in \mathbb{R}_{+} \times \mathbb{R}$, on

$$
\left\{\omega:(t, x) \in Z(N, n, K, \beta), N \geq N_{[5.6}\right],
$$

$$
\begin{aligned}
& Q_{T, \delta}\left(s, s^{\prime}, t^{\prime}, x^{\prime}\right) \\
& \leq \Phi \text { 5.6 }(K)\left[a_{n}^{-2 \varepsilon_{0}}+2^{4 \Lambda}[\overline{5.6}]\left|s^{\prime}-s\right|^{1-\frac{\eta_{1}}{2}}\left[\bar{\delta}_{N}^{\left(\gamma \gamma_{m}-3 / 2\right) \wedge 0}+a_{n}^{2 \beta \gamma} \bar{\delta}_{N}^{(\gamma-3 / 2) \wedge 0}\right.\right. \\
& \left.+1\left(\delta<\vec{d}_{N}^{2}\right) \delta^{-3 / 2}\left[\vec{d}_{N}^{2 \gamma \tilde{\gamma}_{m}}+a_{n}^{2 \beta \gamma} \vec{d}_{N}^{2 \gamma}\right]\right] \\
& \text { for all } s \leq t \leq t^{\prime}, s^{\prime} \leq t^{\prime} \leq T_{K} \text {, and }\left|x^{\prime}\right| \leq K+1 \text {. }
\end{aligned}
$$

Here $d=d\left((t, x),\left(t^{\prime}, x^{\prime}\right)\right), \bar{d}_{N}=d \vee 2^{-N}$ and $\bar{\delta}_{N}=\delta \vee \bar{d}_{N}^{2}$. Moreover $N_{5.6}$ is stochastically bounded uniformly in $(n, \beta)$.

Proof. Let $\xi=1-(2 R)^{-1}$ and define $N_{5.6}=N_{1}\left(m, n, \xi, \varepsilon_{0}, K, \beta\right)$ so that the last statement is immediate from $\left(P_{m}\right)$. We may assume $s \vee s^{\prime} \equiv \bar{s} \geq \delta$, or the left-hand side is 0 . Let $\underline{s}=s \wedge s^{\prime}$. We again use Lemma 5.2 to bound $|u(r, y)|$ in the integrand defining $Q_{T, \delta}$ and the maximum with 
$\sqrt{a_{n}}$ can be ignored as it is less than $\sqrt{t^{\prime}-r}$ in the calculation below. So for $\omega$ as in (5.16) and $s, t, s^{\prime}, t^{\prime}, x^{\prime}$ as in (5.17), we have (note that $r \leq \bar{s} \leq t^{\prime} \leq T_{K}$ so that Lemma 5.2 applies)

$$
\begin{aligned}
& Q_{T, \delta}\left(s, s^{\prime}, t^{\prime}, x^{\prime}\right) \\
& \leq q_{\underline{5.2} \int_{(\underline{s}-\delta)^{+}}^{\bar{s}-\delta}} \int p_{t^{\prime}-r}^{\prime}\left(y-x^{\prime}\right)^{2} e^{2 R_{1} K} e^{2\left(R_{1}+1\right)|y-x|}\left[2^{-N} \vee\left(\sqrt{t^{\prime}-r}+|y-x|\right)\right]^{2 \gamma \xi} \\
& \times\left[\left(2^{-N} \vee\left(\sqrt{t^{\prime}-r}+|y-x|\right)\right)^{\tilde{\gamma}_{m}-1}+a_{n}^{\beta}\right]^{2 \gamma} d y d r .
\end{aligned}
$$

Use Lemma 4.2, the inequality

$$
2^{-N} \vee\left(\sqrt{t^{\prime}-r}+|y-x|\right) \leq\left(2^{-N} \vee\left|x-x^{\prime}\right|\right)+\sqrt{t^{\prime}-r}+\left|y-x^{\prime}\right| \leq \bar{d}_{N}+\sqrt{t^{\prime}-r}+\left|y-x^{\prime}\right|
$$

and $e^{2\left(R_{1}+1\right)|y-x|} \leq c_{0}(K) e^{2\left(R_{1}+1\right)\left|y-x^{\prime}\right|}$ to bound the above by

$$
\begin{aligned}
& c_{1}(K) C_{5.2} \int_{(\underline{s}-\delta)^{+}}^{\bar{s}-\delta} \int\left(t^{\prime}-r\right)^{-1} p_{2\left(t^{\prime}-r\right)}(z)^{2} e^{2\left(R_{1}+1\right)|z|}\left[\vec{d}_{N}^{2 \gamma \xi}+\left(t^{\prime}-r\right)^{\gamma \xi}+|z|^{2 \gamma \xi}\right] \\
& \times\left[\left(\vec{d}_{N}^{2 \gamma\left(\tilde{\gamma}_{m}-1\right)}+\left(t^{\prime}-r\right)^{\gamma\left(\tilde{\gamma}_{m}-1\right)}+|z|^{2 \gamma\left(\tilde{\gamma}_{m}-1\right)}\right)+a_{n}^{2 \beta \gamma}\right] d z d r \\
& \leq c_{2}(K) q_{5.2} \int_{(\underline{s}-\delta)^{+}}^{\bar{s}-\delta}\left(t^{\prime}-r\right)^{-3 / 2}\left[\vec{d}_{N}^{\gamma \xi \xi}+\left(t^{\prime}-r\right)^{\gamma \xi}\right]\left[\left(\vec{d}_{N}^{2 \gamma\left(\tilde{\gamma}_{m}-1\right)}+\left(t^{\prime}-r\right)^{\gamma\left(\tilde{\gamma}_{m}-1\right)}\right)+a_{n}^{2 \beta \gamma}\right] d r \\
& \leq c_{2}(K) q_{\underline{5.2}}\left\{\int_{(\underline{s}-\delta)^{+}}^{\bar{s}-\delta} 1\left(r \leq t^{\prime}-\bar{d}_{N}^{2}\right)\left[\left(t^{\prime}-r\right)^{\gamma\left(\tilde{\gamma}_{m}+\xi-1\right)-3 / 2}+a_{n}^{2 \beta \gamma}\left(t^{\prime}-r\right)^{\gamma \xi-3 / 2}\right] d r\right. \\
& \left.+\int_{(\underline{s}-\delta)^{+}}^{\bar{s}-\delta} 1\left(r>t^{\prime}-\vec{d}_{N}^{2}\right)\left(t^{\prime}-r\right)^{-3 / 2} d r\left[\vec{d}_{N}^{2 \gamma\left(\tilde{\gamma}_{m}+\xi-1\right)}+a_{n}^{2 \beta \gamma} \vec{d}_{N}^{2 \gamma \xi}\right]\right\} \\
& (5.19) \equiv c_{2}(K) C_{5.2}\left\{J_{1}+J_{2}\right\} \text {. }
\end{aligned}
$$

Now

$$
\begin{aligned}
\int_{(\underline{s}-\delta)^{+}}^{\bar{s}-\delta} 1\left(r>t^{\prime}-\vec{d}_{N}^{2}\right)\left(t^{\prime}-r\right)^{-3 / 2} d r & \leq 1\left(\delta<\vec{d}_{N}^{2}\right)\left[\left(t^{\prime}-\bar{s}+\delta\right)^{-3 / 2}\left|s^{\prime}-s\right| \wedge 2\left(t^{\prime}-\bar{s}+\delta\right)^{-1 / 2}\right] \\
& \leq 1\left(\delta<\vec{d}_{N}^{2}\right) 2 \delta^{-3 / 2}\left(\left|s^{\prime}-s\right| \wedge \delta\right)
\end{aligned}
$$

and so

$$
\begin{aligned}
J_{2} & \leq 1\left(\delta<\vec{d}_{N}^{2}\right) 2 \delta^{-3 / 2}\left(\left|s^{\prime}-s\right| \wedge \delta\right) \vec{d}_{N}^{(-2 \gamma(1-\xi))}\left[\vec{d}_{N}^{2 \gamma \tilde{\gamma}_{m}}+a_{n}^{2 \beta \gamma} \vec{d}_{N}^{2 \gamma}\right] \\
& \leq c_{3}(K) 1\left(\delta<\vec{d}_{N}^{2}\right) \delta^{-3 / 2}\left(\left|s^{\prime}-s\right| \wedge \delta\right)^{1-\frac{\eta_{1}}{2}}\left[\vec{d}_{N}^{2 \gamma \tilde{\gamma}_{m}}+a_{n}^{2 \beta \gamma} \vec{d}_{N}^{2 \gamma}\right]
\end{aligned}
$$

In the last line we used $\gamma(1-\xi) \leq 1-\xi \leq(2 R)^{-1}<\eta_{1} / 2$ and $\left|s^{\prime}-s\right| \leq 2 K$.

Turning to $J_{1}$, let $p=\gamma\left(\tilde{\gamma}_{m}+\xi-1\right)-3 / 2$ or $\gamma \xi-3 / 2$ for $0 \leq m-1 \leq \bar{m}$. Our bounds on $\gamma$ and $\xi$ (both are bigger than 3/4) imply $p \in[\gamma \xi-3 / 2,1 / 2] \subset[-15 / 16,1 / 2]$. If $p^{\prime}=p \wedge 0$ and 
$0 \leq \varepsilon \leq-p^{\prime}$, then

$$
\begin{aligned}
I(p) & \equiv \int_{(\underline{s}-\delta)^{+}}^{\bar{s}-\delta} 1\left(r \leq t^{\prime}-\bar{d}_{N}^{2}\right)\left(t^{\prime}-r\right)^{p} d r \\
& \leq \int_{(\underline{s}-\delta)^{+}}^{\bar{s}-\delta} 1\left(r \leq t^{\prime}-\bar{d}_{N}^{2}\right) \sqrt{K}\left(t^{\prime}-r\right)^{p^{\prime}} d r \\
& \leq \sqrt{K} \min \left(\left|s^{\prime}-s\right| \bar{\delta}_{N}^{p^{\prime}}, \int_{0}^{\left|s^{\prime}-s\right|} u^{p^{\prime}} d u\right) \\
& \left.\leq 16 \sqrt{K}\left|s^{\prime}-s\right|^{p^{\prime}+1} \min \left(\left(\frac{\left|s^{\prime}-s\right|}{\bar{\delta}_{N}}\right)^{-p^{\prime}}, 1\right) \quad \text { (use } p^{\prime} \geq-15 / 16\right) \\
& \leq 16 \sqrt{K}\left|s^{\prime}-s\right|^{p^{\prime}+1}\left(\frac{\left|s^{\prime}-s\right|}{\bar{\delta}_{N}}\right)^{-p^{\prime}-\varepsilon} \\
& =16 \sqrt{K}\left|s^{\prime}-s\right|^{1-\varepsilon} \bar{\delta}_{N}^{\varepsilon+p^{\prime}} .
\end{aligned}
$$

Define $q=p+\gamma(1-\xi)$, so that $q=\gamma \tilde{\gamma}_{m}-3 / 2$ or $\gamma-3 / 2$.

Case 1. $q \leq 0$.

Then $p^{\prime}=p \leq 0$. If $\varepsilon=\gamma(1-\xi) \leq(2 R)^{-1}<\eta_{1} / 2$, then $\varepsilon+p^{\prime}=q \leq 0$ and so (5.22) applies, and gives

$$
I(p) \leq 16 \sqrt{K}\left|s^{\prime}-s\right|^{1-\varepsilon} \bar{\delta}_{N}^{q} \leq 16 K\left|s^{\prime}-s\right|^{1-\frac{\eta_{1}}{2}} \bar{\delta}_{N}^{q}
$$

Case 2. $q>0$

Then $p^{\prime}=(q-\gamma(1-\xi)) \wedge 0 \geq-\gamma(1-\xi)$. Let $\varepsilon=-p^{\prime} \leq \gamma(1-\xi) \leq(2 R)^{-1}<\eta_{1} / 2$ in (5.22) and conclude

$$
I(p) \leq 16 \sqrt{K}\left|s^{\prime}-s\right|^{1-\varepsilon} \leq 16 K\left|s^{\prime}-s\right|^{1-\frac{\eta_{1}}{2}} .
$$

In either case we have shown that $I(p) \leq 16 K\left|s^{\prime}-s\right|^{1-\frac{\eta_{1}}{2}} \bar{\delta}_{N}^{q \wedge 0}$. This gives

$$
J_{1} \leq c_{4}(K)\left|s^{\prime}-s\right|^{1-\frac{\eta_{1}}{2}}\left[\bar{\delta}_{N}^{\left(\gamma \tilde{\gamma}_{m}-3 / 2\right) \wedge 0}+a_{n}^{2 \beta \gamma} \bar{\delta}_{N}^{(\gamma-3 / 2) \wedge 0}\right] .
$$

Put (5.21) and (5.25) into (5.19) and use (5.15) to complete the proof.

Notation. $d\left((s, t, x),\left(s^{\prime}, t^{\prime}, x^{\prime}\right)\right)=\sqrt{\left|s^{\prime}-s\right|}+\sqrt{\left|t^{\prime}-t\right|}+\left|x^{\prime}-x\right|$.

Lemma 5.7 Let $c_{0}, c_{1}, c_{2}, k_{0}$ be positive (universal constants), $\eta \in(0,1 / 2)$, and $\Delta: \mathbb{N} \times(0,1] \rightarrow \mathbb{R}_{+}$ satisfy $\Delta\left(n, 2^{-N+1}\right) \leq k_{0} \Delta\left(n, 2^{-N}\right)$ for all $n, N \in \mathbb{N}$. For $n \in \mathbb{N}$ and $\tau$ in a set $S$ assume $\left\{Y_{\tau, n}(s, t, x):(s, t, x) \in \mathbb{R}_{+}^{2} \times \mathbb{R}\right\}$ is a real-valued continuous process. Assume for each $(n, \tau), K \in \mathbb{N}$, and $\beta \in[0,1 / 2]$, there is an $N_{0}(\omega)=N_{0}(n, \eta, K, \tau, \beta)(\omega) \in \mathbb{N}$ a.s., stochastically bounded uniformly in $(n, \tau, \beta)$, such that for any $N \in \mathbb{N},(t, x) \in \mathbb{R}_{+} \times \mathbb{R}$, and $s \leq K$, if $\tilde{d}=d\left((s, t, x),\left(s^{\prime}, t^{\prime}, x^{\prime}\right)\right) \leq$ $2^{-N}$, then

$$
\begin{aligned}
& P\left(\left|Y_{\tau, n}(s, t, x)-Y_{\tau, n}\left(s^{\prime}, t^{\prime}, x^{\prime}\right)\right|>\tilde{d}^{1-\eta} \Delta\left(n, 2^{-N}\right),(t, x) \in Z(N, n, K, \beta), N \geq N_{0}, t^{\prime} \leq T_{K}\right) \\
& \leq c_{0} \exp \left(-c_{1} \tilde{d}^{-\eta c_{2}}\right) .
\end{aligned}
$$

Then there is an $N_{0}^{\prime}=N_{0}^{\prime}(n, \eta, K, \tau, \beta) \in \mathbb{N}$ a.s., also stochastically bounded uniformly in $(n, \tau, \beta)$, such that for all $N \geq N_{0}^{\prime}(\omega),(t, x) \in Z(N, n, K, \beta)(\omega), \tilde{d}=d\left((s, t, x),\left(s^{\prime}, t^{\prime}, x^{\prime}\right)\right) \leq 2^{-N}, s \leq K$ and $t^{\prime} \leq T_{K}$

$$
\left|Y_{\tau, n}(s, t, x)-Y_{\tau, n}\left(s^{\prime}, t^{\prime}, x^{\prime}\right)\right| \leq 2^{7} k_{0}^{3} \tilde{d}^{1-\eta} \Delta\left(n, 2^{-N}\right) .
$$


Proof. Let

$$
\begin{array}{r}
M_{\ell, N}=M_{\ell, N}^{\tau, n, K, \beta}=\max \left\{\left|Y_{\tau, n}\left((i+e) 2^{-2 \ell},(j+f) 2^{-2 \ell},(k+g) 2^{-\ell}\right)-Y_{\tau, n}\left(i 2^{-2 \ell}, j 2^{-2 \ell}, k 2^{-\ell}\right)\right|:\right. \\
\left(j 2^{-2 \ell}, k 2^{-\ell}\right) \in Z(N, n, K+1, \beta), e, f=-4,-3, \ldots 4, i 2^{-2 \ell} \leq K+1, \\
\left.g=-2,-1, \ldots, 2,(j+f) 2^{-2 \ell} \leq T_{K+1}, i, j, i+e, j+f \in \mathbb{Z}_{+}, k \in \mathbb{Z}\right\},
\end{array}
$$

and

$$
A_{N}=\left\{\omega: \exists \ell \geq N+3 \text { s. t. } M_{\ell, N} \geq 2^{(3-\ell)(1-\eta)} \Delta\left(n, 2^{-N}\right), N \geq N_{0}(n, \eta, K+1, \tau, \beta)\right\} .
$$

For $i, j, k, e, f, g$ as in the definition of $M_{\ell, N}$ and $\ell \geq N+3$,

$$
d\left(\left((i+e) 2^{-2 \ell},(j+f) 2^{-2 \ell},(k+g) 2^{-\ell}\right),\left(i 2^{-2 \ell}, j 2^{-2 \ell}, k 2^{-\ell}\right)\right) \leq 2^{3-\ell} \leq 2^{-N} .
$$

Therefore (5.26) implies that for some $c_{1}^{\prime}=c_{1}^{\prime}(\eta)>0$,

$$
\begin{aligned}
P\left(\cup_{N^{\prime}=N}^{\infty} A_{N^{\prime}}\right) & \leq \sum_{N^{\prime}=N}^{\infty} \sum_{\ell=N^{\prime}+3}^{\infty} 5 \cdot 9^{2}\left[2^{2 \ell}(K+1)+1\right]^{2}\left[2^{\ell+1}(K+1)+1\right] c_{0} \exp \left(-c_{1}\left(2^{3-\ell}\right)^{-\eta c_{2}}\right) \\
& \leq c_{3}(K) \exp \left(-c_{1}^{\prime} 2^{N \eta c_{2}}\right) .
\end{aligned}
$$

Let

$$
N_{2}=N_{2}(n, \eta, K, \tau, \beta)=\min \left\{N: \omega \in \cap_{N^{\prime}=N}^{\infty} A_{N^{\prime}}^{c}\right\} .
$$

The above implies that

$$
P\left(N_{2}>N\right)=P\left(\cup_{N^{\prime}=N}^{\infty} A_{N^{\prime}}\right) \leq c_{3}(K) \exp \left(-c_{1}^{\prime} 2^{N \eta c_{2}}\right)
$$

Define

$$
N_{0}^{\prime}(n, \eta, K, \tau, \beta)=\left(N_{0}(n, \eta, K+1, \tau, \beta) \vee N_{2}(n, \eta, K, \tau, \beta)\right)+3 .
$$

$N_{0}^{\prime}$ is stochastically bounded uniformly in $(n, \tau, \beta)$ by (5.27) and the corresponding property of $N_{0}$. Assume

$$
N \geq N_{0}^{\prime},(t, x) \in Z(N, n, K, \beta), d\left((s, t, x)\left(s^{\prime}, t^{\prime}, x^{\prime}\right)\right) \leq 2^{-N}, s \leq K \text { and } t^{\prime} \leq T_{K}
$$

Define dyadic approximations by $s_{\ell}=\left\lfloor 2^{2 \ell} s\right\rfloor 2^{-2 \ell}, t_{\ell}=\left\lfloor 2^{2 \ell} t\right\rfloor 2^{-2 \ell}, x_{\ell}=\operatorname{sgn}(x)\left\lfloor 2^{\ell}|x|\right\rfloor 2^{-\ell}$, and similarly define $s_{\ell}^{\prime}, t_{\ell}^{\prime}$ and $x_{\ell}^{\prime}$ for $\left(s^{\prime}, t^{\prime}, x^{\prime}\right)$. Choose $\left(\hat{t}_{0}, \hat{x}_{0}\right)$ as in the definition of $(t, x) \in Z(N, n, K, \beta)$. Then $\left|x_{\ell}\right| \leq|x| \leq K,\left|x_{\ell}^{\prime}\right| \leq\left|x^{\prime}\right| \leq K+1, s_{\ell}^{\prime} \vee s_{\ell} \leq s^{\prime} \vee s \leq K, t_{\ell}^{\prime} \vee t_{\ell} \leq t^{\prime} \vee t \leq T_{K}$, and if $\ell \geq N$,

$$
\begin{aligned}
d\left(\left(t_{\ell}^{\prime}, x_{\ell}^{\prime}\right),\left(\hat{t}_{0}, \hat{x}_{0}\right)\right) & \leq d\left(\left(t_{\ell}^{\prime}, x_{\ell}^{\prime}\right),\left(t^{\prime}, x^{\prime}\right)\right)+d\left(\left(t^{\prime}, x^{\prime}\right),(t, x)\right)+d\left((t, x),\left(\hat{t}_{0}, \hat{x}_{0}\right)\right) \\
& \leq \sqrt{\left|t_{\ell}^{\prime}-t^{\prime}\right|}+\left|x_{\ell}^{\prime}-x^{\prime}\right|+2^{1-N} \\
& \leq 2^{2-N} .
\end{aligned}
$$

This proves that

$$
\left(t_{\ell}^{\prime}, x_{\ell}^{\prime}\right) \in Z(N-2, n, K+1, \beta) \subset Z(N-3, n, K+1, \beta) \text { for all } \ell \geq N
$$


and even more simply one gets

$$
\left(t_{\ell}, x_{\ell}\right) \in Z(N-3, n, K, \beta) \text { for all } \ell \geq N .
$$

In addition, the fact that $N \geq N_{0}^{\prime}$ implies $\omega \in A_{N-3}^{c}$ and $N-3 \geq N_{0}$, which in turn implies

$$
M_{\ell, N-3} \leq 2^{(3-\ell)(1-\eta)} \Delta\left(n, 2^{-(N-3)}\right) \text { for all } \ell \geq N .
$$

Choose $N^{\prime} \geq N$ such that $2^{-N^{\prime}-1}<d\left((s, t, x),\left(s^{\prime}, t^{\prime}, x^{\prime}\right)\right) \equiv \tilde{d} \leq 2^{-N^{\prime}}$. Then $\left|x^{\prime}-x\right| \leq 2^{-N^{\prime}}$ which implies $x_{N^{\prime}}^{\prime}=x_{N^{\prime}}+g 2^{-N^{\prime}}$ for $g \in\{-1,0,1\}$. Similarly $s_{N^{\prime}}^{\prime}=s_{N^{\prime}}+e 2^{-2 N^{\prime}}$ and $t_{N^{\prime}}^{\prime}=t_{N^{\prime}}+f 2^{-2 N^{\prime}}$ for $e, f \in\{-1,0,1\}$. In addition, $s_{\ell}=s_{\ell-1}+e 4^{-\ell}, t_{\ell}=t_{\ell-1}+f 4^{-\ell}$, and $x_{\ell}=x_{\ell-1}+g 2^{-\ell}$ for some $e, f \in\{0, \ldots, 3\}$ and $g \in\{-1,0,1\}$, and similarly for $s_{\ell}^{\prime}, t_{\ell}^{\prime}$ and $x_{\ell}^{\prime}$. Let $w_{\ell}=\left(s_{\ell}, t_{\ell}, x_{\ell}\right)$ and $w_{\ell}^{\prime}=\left(s_{\ell}^{\prime}, t_{\ell}^{\prime}, x_{\ell}^{\prime}\right)$. Now use (5.29), (5.30), (5.31), the definition of $M_{\ell, N-3}$ and the continuity of $Y_{\tau, n}$ to see that for $(s, t, x),\left(s^{\prime}, t^{\prime}, x^{\prime}\right)$ as in (5.28),

$$
\begin{aligned}
& \left|Y_{\tau, n}(s, t, x)-Y_{\tau, n}\left(s^{\prime}, t^{\prime}, x^{\prime}\right)\right| \\
& \leq\left|Y_{\tau, n}\left(w_{N^{\prime}}^{\prime}\right)-Y_{\tau, n}\left(w_{N^{\prime}}\right)\right|+\sum_{\ell=N^{\prime}+1}^{\infty}\left|Y_{\tau, n}\left(w_{\ell}^{\prime}\right)-Y_{\tau, n}\left(w_{\ell-1}^{\prime}\right)\right|+\left|Y_{\tau, n}\left(w_{\ell}\right)-Y_{\tau, n}\left(w_{\ell-1}\right)\right| \\
& \leq M_{N^{\prime}, N-3}+\sum_{\ell=N^{\prime}+1}^{\infty} 2 M_{\ell, N-3} \\
& \leq\left[2^{\left(3-N^{\prime}\right)(1-\eta)}+2 \sum_{\ell=N^{\prime}+1}^{\infty} 2^{(3-\ell)(1-\eta)}\right] \Delta\left(n, 2^{-(N-3)}\right) \quad(\text { by (5.31) }) \\
& \leq(36) 2^{-N^{\prime}(1-\eta)} \Delta\left(n, 2^{-(N-3)}\right) \\
& \leq 2^{7} k_{0}^{3} \tilde{d}^{1-\eta} \Delta\left(n, 2^{-N}\right) .
\end{aligned}
$$

Notation. Introduce

$$
\begin{aligned}
\bar{\Delta}_{u_{1}^{\prime}}\left(m, n, \alpha, \varepsilon_{0}, 2^{-N}\right)=a_{n}^{-\varepsilon_{0}}[ & a_{n}^{-3 \alpha / 4} 2^{-N \gamma \tilde{\gamma}_{m}}+\left(a_{n}^{\alpha / 2} \vee 2^{-N}\right)^{\left(\gamma_{m+1}-2\right) \wedge 0} \\
& \left.+a_{n}^{-3 \alpha / 4+\beta \gamma}\left(a_{n}^{\alpha / 2} \vee 2^{-N}\right)^{\gamma}\right]
\end{aligned}
$$

We often suppress the dependence on $\varepsilon_{0}$ and $\alpha$.

Proposition 5.8 Let $0 \leq m \leq \bar{m}+1$ and assume $\left(P_{m}\right)$. For any $n \in \mathbb{N}, \eta_{1} \in(0,1 / 2), \varepsilon_{0} \in(0,1)$, $K \in \mathbb{N}^{\geq K_{1}}, \alpha \in[0,1]$, and $\beta \in[0,1 / 2]$, there is an $N_{5.8}=N\left[5.8\left(m, n, \eta_{1}, \varepsilon_{0}, K, \alpha, \beta\right)(\omega) \in \mathbb{N} \geq 2\right.$ a.s. such that for all $N \geq N_{5.8},(t, x) \in Z(N, n, K, \beta), t^{\prime} \leq T_{K}, s \leq K$,

$$
\begin{aligned}
& d\left((s, t, x),\left(s^{\prime}, t^{\prime}, x^{\prime}\right)\right) \leq 2^{-N} \text { implies that } \\
& \left|F_{a_{n}^{\alpha}}(s, t, x)-F_{a_{n}^{\alpha}}\left(s^{\prime}, t^{\prime}, x^{\prime}\right)\right| \leq 2^{-86} d\left((s, t, x),\left(s^{\prime}, t^{\prime}, x^{\prime}\right)\right)^{1-\eta_{1}} \bar{\Delta}_{u_{1}^{\prime}}\left(m, n, \alpha, \varepsilon_{0}, 2^{-N}\right) .
\end{aligned}
$$

Moreover $N_{5.8}$ is stochastically bounded, uniformly in $(n, \alpha, \beta)$. 
Proof. Let $R=33 \eta_{1}^{-1}$ and choose $\eta_{0} \in\left(R^{-1}, \eta_{1} / 32\right)$. Let $d=d\left((t, x),\left(t^{\prime}, x^{\prime}\right)\right), \tilde{d}=d+\sqrt{\left|s^{\prime}-s\right|}$, $\bar{d}_{N}=d \vee 2^{-N}, \bar{\delta}_{n, N}=a_{n}^{\alpha} \vee \bar{d}_{N}^{2}$ and

$$
Q_{a_{n}^{\alpha}}\left(s, t, x, s^{\prime}, t^{\prime}, x^{\prime}\right)=Q_{T, a_{n}^{\alpha}}\left(s, s^{\prime}, t^{\prime}, x^{\prime}\right)+\sum_{i=1}^{2} Q_{S, i, a_{n}^{\alpha}, \eta_{0}}\left(s, t, x, t^{\prime}, x^{\prime}\right) .
$$

By Lemmas 5.4, 5.5 and 5.6 there are is a $c_{1}\left(K, \eta_{1}\right)$ and $N_{2}=N_{2}\left(m, n, \eta_{1}, \varepsilon_{0}, K, \beta\right)(\omega)$ stochastically bounded uniformly in $(n, \beta)$, such that for all $N \in \mathbb{N}$ and $(t, x)$, on

$$
\begin{gathered}
\left\{\omega:(t, x) \in Z(N, n, K+1, \beta), N \geq N_{2}\right\}, \\
R_{0}^{\gamma} Q_{a_{n}^{\alpha}}\left(s, t, x, s^{\prime}, t^{\prime}, x^{\prime}\right)^{1 / 2} \\
\leq c_{1}\left(K, \eta_{1}\right)\left[a_{n}^{-\varepsilon_{0}}+2^{2 N_{2}}\right] \tilde{d}^{1-\eta_{1} / 2}\left\{a_{n}^{-3 \alpha / 4}\left[\bar{d}_{N}^{\gamma \tilde{\gamma}_{m}}+a_{n}^{\beta \gamma} \bar{d}_{N}^{\gamma}\right]\right. \\
\left.+\left(\sqrt{\bar{\delta}_{n, N}}\right)^{\left(\gamma \gamma_{m}-3 / 2\right) \wedge 0}+a_{n}^{\beta \gamma}\left(\sqrt{\bar{\delta}_{n, N}}\right)^{(\gamma-3 / 2) \wedge 0}\right\} \\
\quad \text { for all } s \leq t \leq t^{\prime}, s^{\prime} \leq t^{\prime} \leq T_{K},\left|x^{\prime}\right| \leq K+2 .
\end{gathered}
$$

Let $N_{3}=\left(33 / \eta_{1}\right)\left[N_{2}+N_{4}\left(K, \eta_{1}\right)\right]$, where $N_{4}\left(K, \eta_{1}\right)$ is chosen large enough so that

$$
\begin{aligned}
c_{1}\left(K, \eta_{1}\right)\left[a_{n}^{-\varepsilon_{0}}+2^{2 N_{2}}\right] 2^{-\eta_{1} N_{3} / 4} & \leq c_{1}\left(K, \eta_{1}\right)\left[a_{n}^{-\varepsilon_{0}}+2^{2 N_{2}}\right] 2^{-8 N_{2}-8 N_{4}} \\
& \leq a_{n}^{-\varepsilon_{0}} 2^{-104} .
\end{aligned}
$$

Let

$$
\begin{aligned}
\Delta\left(m, n, \bar{d}_{N}\right)=2^{-100} a_{n}^{-\varepsilon_{0}}\left\{a_{n}^{-3 \alpha / 4}\right. & {\left[\bar{d}_{N}^{\gamma \tilde{\gamma}_{m}}+a_{n}^{\beta \gamma} \bar{d}_{N}^{\gamma}\right] } \\
& \left.+\left(\sqrt{\bar{\delta}_{n, N}}\right)^{\left(\gamma \gamma_{m}-3 / 2\right) \wedge 0}+a_{n}^{\beta \gamma}\left(\sqrt{\bar{\delta}_{n, N}}\right)^{(\gamma-3 / 2) \wedge 0}\right\} .
\end{aligned}
$$

Let $N^{\prime} \in \mathbb{N}$ and assume $\tilde{d} \leq 2^{-N^{\prime}}$. Use (5.34) and (5.35) to see that on

$$
\left\{\omega:(t, x) \in Z(N, n, K+1, \beta), N \geq N_{3}, N^{\prime} \geq N_{3}\right\}
$$

(which implies $\left|x^{\prime}\right| \leq K+2$ ),

$$
\begin{aligned}
R_{0}^{\gamma} Q_{a_{n}^{\alpha}}\left(s, t, x, s^{\prime}, t^{\prime}, x^{\prime}\right)^{1 / 2} & \leq c_{1}\left(K, \eta_{1}\right)\left[a_{n}^{-\varepsilon_{0}}+2^{2 N_{2}}\right] 2^{-\eta_{1} N^{\prime} / 4} \tilde{d}^{1-\left(3 \eta_{1} / 4\right)} 2^{100} a_{n}^{\varepsilon_{0}} \Delta\left(m, n, \bar{d}_{N}\right) \\
& \leq \tilde{d}^{1-\left(3 \eta_{1} / 4\right)} \frac{\Delta\left(m, n, \bar{d}_{N}\right)}{16} \quad \text { for all } s \leq t \leq t^{\prime}, s^{\prime} \leq t^{\prime} \leq T_{K},\left|x^{\prime}\right| \leq K+2 .
\end{aligned}
$$

Combine this with (5.6), (3.2), the definition of $Q_{a_{n}^{\alpha}}$, and the Dubins-Schwarz theorem, to conclude that for $s \leq t \leq t^{\prime}, s^{\prime} \leq t^{\prime}, d\left((s, t, x),\left(s^{\prime}, t^{\prime}, x^{\prime}\right)\right) \leq 2^{-N^{\prime}}$,

$$
\begin{aligned}
& P\left(\left|F_{a_{n}^{\alpha}}(s, t, x)-F_{a_{n}^{\alpha}}\left(s^{\prime}, t^{\prime}, x^{\prime}\right)\right| \geq d\left((s, t, x),\left(s^{\prime}, t^{\prime}, s^{\prime}\right)\right)^{1-\eta_{1}} \Delta\left(m, n, \bar{d}_{N}\right) / 8,\right. \\
& \left.\quad(t, x) \in Z(N, n, K+1, \beta), N^{\prime} \wedge N \geq N_{3}, t^{\prime} \leq T_{K}\right) \\
& \leq 2 P\left(\sup _{u \leq \tilde{d}^{2-\left(3 \eta_{1} / 2\right)}\left(\Delta\left(m, n, \bar{d}_{N}\right) / 16\right)^{2}}|B(u)| \geq \tilde{d}^{1-\eta_{1}} \frac{\Delta\left(m, n, \bar{d}_{N}\right)}{16}\right) \\
& \leq 2 P\left(\sup _{u \leq 1}|B(u)| \geq \tilde{d}^{-\eta_{1} / 4}\right) \\
(5.37) & \leq c_{0} \exp \left(-\tilde{d}^{-\eta_{1} / 2} / 2\right) .
\end{aligned}
$$


Here $B(u)$ is a one-dimensional Brownian motion.

To handle $s>t$, recall that $F_{a_{n}^{\alpha}}(s, t, x)=F_{a_{n}^{\alpha}}(s, s \vee t, x)$. One easily checks that

$$
\sqrt{\left|s \vee t-s^{\prime} \vee t^{\prime}\right|} \leq \sqrt{\left|s-s^{\prime}\right|}+\sqrt{\left|t-t^{\prime}\right|}
$$

and hence $d\left((s, s \vee t, x),\left(s^{\prime}, s^{\prime} \vee t^{\prime}, x^{\prime}\right)\right) \leq 2 d\left((s, t, x),\left(s^{\prime}, t^{\prime}, x^{\prime}\right)\right) \equiv 2 \tilde{d}$. So (5.37) implies that for $t \leq t^{\prime}$ and all $s, s^{\prime}, x^{\prime}$, if $d\left((s, t, x),\left(s^{\prime}, t^{\prime}, x^{\prime}\right)\right) \leq 2^{-N^{\prime}-1}$, then

$$
\begin{aligned}
& P\left(\left|F_{a_{n}^{\alpha}}(s, t, x)-F_{a_{n}^{\alpha}}\left(s^{\prime}, t^{\prime}, x^{\prime}\right)\right| \geq d\left((s, t, x),\left(s^{\prime}, t^{\prime}, x^{\prime}\right)\right)^{1-\eta_{1}} \Delta\left(m, n, \bar{d}_{N}\right) / 4,\right. \\
& \left.(t, x) \in Z(N, n, K+1, \beta), t^{\prime} \leq T_{K}, N^{\prime}+1 \geq N_{3}+1, N \geq N_{3}\right) \\
& \leq c_{0} \exp \left(-\tilde{d}^{-\eta_{1} / 2} / 2\right) .
\end{aligned}
$$

If $(t, x) \in Z(N, n, K, \beta), t^{\prime} \leq t$ and $d=d\left((t, x),\left(t^{\prime}, x^{\prime}\right)\right) \leq 2^{-N}$, then we claim that $\left(t^{\prime}, x^{\prime}\right) \in$ $Z(N-1, n, K+1, \beta)$. Indeed if $\left(\hat{t}_{0}, \hat{x}_{0}\right)$ is as in the definition of $(t, x) \in Z(N, n, K, \beta)$, then $d\left(\left(\hat{t}_{0}, \hat{x}_{0}\right),\left(t^{\prime}, x^{\prime}\right)\right) \leq 2^{-(N-1)}$. Also $\left|x^{\prime}\right| \leq K+1, t^{\prime} \leq t \leq T_{K}$, and the claim follows. Note also that as $d \leq 2^{-N}$, we have $\bar{d}_{N}=2^{-N}$. An elementary argument using $\gamma \gamma_{k} \leq 2$ for $k \leq m-1 \leq \bar{m}$ and $\gamma \tilde{\gamma}_{k} \leq 2$, shows that

$$
\Delta\left(m, n, 2^{-N}\right) \geq 4^{-1} \Delta\left(m, n, 2^{-(N-1)}\right) .
$$

So, by interchanging $\left(t^{\prime}, x^{\prime}\right)$ and $(t, x)$, and replacing $N$ with $N-1$, (5.38) and (5.39) imply that for $t^{\prime} \leq t, \tilde{d} \leq 2^{-N^{\prime}}$ and $d \leq 2^{-N}$,

$$
\begin{gathered}
P\left(\left|F_{a_{n}^{\alpha}}(s, t, x)-F_{a_{n}^{\alpha}}\left(s^{\prime}, t^{\prime}, x^{\prime}\right)\right| \geq \tilde{d}^{1-\eta_{1}} \Delta\left(m, n, 2^{-N}\right),\right. \\
\left.\quad(t, x) \in Z(N, n, K, \beta), N^{\prime} \wedge N \geq N_{3}+1\right) \\
\leq P\left(\left|F_{a_{n}^{\alpha}}(s, t, x)-F_{a_{n}^{\alpha}}\left(s^{\prime}, t^{\prime}, x^{\prime}\right)\right| \geq \tilde{d}^{1-\eta_{1}} \Delta\left(m, n, 2^{-(N-1)}\right) / 4,\right. \\
\left.\quad\left(t^{\prime}, x^{\prime}\right) \in Z(N-1, n, K+1, \beta), N^{\prime} \geq N_{3}+1, N-1 \geq N_{3}, t \leq T_{K}\right) \\
(5.40) \leq c_{0} \exp \left(-\tilde{d}^{-\eta_{1} / 2} / 2\right) .
\end{gathered}
$$

If $N_{5}\left(m, n, \eta_{1} \varepsilon_{0}, K, \beta\right)(\omega)=N_{3}(\omega)+1$, then $N_{5}$ is stochastically bounded, uniformly in $(n, \beta)$. We have shown, (taking $N^{\prime}=N$ in the above) that for all $(s, t, x),\left(s^{\prime}, t^{\prime}, x^{\prime}\right)$, if $\tilde{d}=d\left((s, t, x),\left(s^{\prime}, t^{\prime}, x^{\prime}\right)\right) \leq$ $2^{-N}$, then

$$
\begin{aligned}
& P\left(\left|F_{a_{n}^{\alpha}}(s, t, x)-F_{a_{n}^{\alpha}}\left(s^{\prime}, t^{\prime}, x^{\prime}\right)\right| \geq \tilde{d}^{1-\eta_{1}} \Delta\left(m, n, 2^{-N}\right),(t, x) \in Z(N, n, K, \beta), N \geq N_{5}, t^{\prime} \leq T_{K}\right) \\
& \leq c_{0} \exp \left(-\tilde{d}^{-\eta_{1} / 2} / 2\right) .
\end{aligned}
$$

Now apply Lemma 5.7 with $\tau=\alpha \in[0,1], Y_{\tau, n}=F_{a_{n}^{\alpha}}$ and $k_{0}=2^{2}$, the latter by (5.39). (5.41) shows that (5.26) holds with $N_{0}=N_{5}$. (The implicit restriction $K \geq K_{1}$ in (5.41) from Lemmas 5.45.6 is illusory as increasing $K$ only strengthens (5.41) $)$.) Therefore there is an $N_{5.8}(\omega)=$ $N_{5.8}\left(m, n, \eta_{1}, \varepsilon_{0}, K, \alpha, \beta\right) \geq 2$, stochastically bounded uniformly in $(n, \alpha, \beta)$, such that for $N \geq$ $N_{5.8},(t, x) \in Z(N, n, K, \beta)$, if $t^{\prime} \leq T_{K}, s \leq K$ and $\tilde{d}=d\left((s, t, x),\left(s^{\prime}, t^{\prime}, x^{\prime}\right)\right) \leq 2^{-N}$, then

$$
\left|F_{a_{n}^{\alpha}}(s, t, x)-F_{a_{n}^{\alpha}}\left(s^{\prime}, t^{\prime}, x^{\prime}\right)\right| \leq 2^{13} \Delta\left(m, n, 2^{-N}\right) \tilde{d}^{1-\eta_{1}} .
$$


Note that

$$
\begin{gathered}
\Delta\left(m, n, 2^{-N}\right)=2^{-100} a_{n}^{-\varepsilon_{0}}\left[a_{n}^{-3 \alpha / 4} 2^{-N \gamma \tilde{\gamma}_{m}}+\left(a_{n}^{\alpha / 2} \vee 2^{-N}\right)^{\left(\gamma \gamma_{m}-3 / 2\right) \wedge 0}\right. \\
\left.\quad+a_{n}^{\beta \gamma}\left(a_{n}^{-3 \alpha / 4} 2^{-N \gamma}+\left(a_{n}^{\alpha / 2} \vee 2^{-N}\right)^{\gamma-3 / 2}\right)\right] \\
\leq 2^{-99} a_{n}^{\varepsilon_{0}}\left[a_{n}^{-3 \alpha / 4} 2^{-N \gamma \tilde{\gamma}_{m}}+\left(a_{n}^{\alpha / 2} \vee 2^{-N}\right)^{\left(\gamma_{m+1}-2\right) \wedge 0}\right. \\
\left.+a_{n}^{\beta \gamma-\frac{3 \alpha}{4}}\left(2^{-N} \vee a_{n}^{\alpha / 2}\right)^{\gamma}\right] .
\end{gathered}
$$

Use this in (5.42) to complete the proof.

Since $F_{\delta}(t, t, x)=-u_{1, \delta}^{\prime}(t, x)$ (see Remark 4.2), the following Corollary is immediate.

Corollary 5.9 Let $0 \leq m \leq \bar{m}+1$ and assume $\left(P_{m}\right)$. Let $n, \eta_{1}, \varepsilon_{0}, K, \alpha$ and $\beta$ be as in Proposition 5.8. For all $N \geq N$ 5.8, $(t, x) \in Z(N, n, K, \beta)$ and $t^{\prime} \leq T_{K}$,

$$
\begin{aligned}
& d\left((t, x),\left(t^{\prime}, x^{\prime}\right)\right) \leq 2^{-N} \text { implies that } \\
& \left|u_{1, a_{n}^{\alpha}}^{\prime}(t, x)-u_{1, a_{n}^{\alpha}}^{\prime}\left(t^{\prime}, x^{\prime}\right)\right| \leq 2^{-85} d\left((t, x),\left(t^{\prime}, x^{\prime}\right)\right)^{1-\eta_{1}} \bar{\Delta}_{u_{1}^{\prime}}\left(m, n, \alpha, \varepsilon_{0}, 2^{-N}\right) .
\end{aligned}
$$

We will need to modify the bound in Lemma 5.6 to control $\left|u_{1, \delta}^{\prime}-u_{1, a_{n}}^{\prime}\right|$. Note that if $\delta \geq a_{n}$ and $s=t-\delta+a_{n}$ then

$$
\begin{aligned}
u_{1, \delta}^{\prime}(t, x)=\frac{d}{d x} P_{\delta}\left(u_{(t-\delta)^{+}}\right)(x) & =\frac{d}{d x} P_{t-s+a_{n}}\left(u_{\left(s-a_{n}\right)^{+}}\right)(x) \\
& =-F_{a_{n}}(s, t, x) \\
& =-F_{a_{n}}\left(t-\delta+a_{n}, t, x\right) .
\end{aligned}
$$

Therefore the key will be a bound on $\left|F_{a_{n}}(s, t, x)-F_{a_{n}}(t, t, x)\right|$ in which the hypothesis (for Proposition 5.8) $\sqrt{t-s} \leq 2^{-N}$ is weakened substantially.

Lemma 5.10 Let $0 \leq m \leq \bar{m}+1$ and assume $\left(P_{m}\right)$. For any $K \in \mathbb{N} \geq K_{1}, R>2, n \in \mathbb{N}, \varepsilon_{0} \in(0,1)$, and $\beta \in[0,1 / 2]$ there is a $\overline{5.10}(K)$ and $N_{5.10}=N_{5.10}\left(m, n, R, \varepsilon_{0}, K, \beta\right)(\omega) \in \mathbb{N}$ a.s. such that for any $\eta_{1} \in\left(R^{-1}, 1 / 2\right), N \in \mathbb{N}$, and $(t, x) \in \mathbb{R}_{+} \times \mathbb{R}$, on

$$
\{\omega:(t, x) \in Z(N, n, K, \beta), N \geq N[\overline{5.10}\},
$$

$$
\begin{aligned}
& Q_{T, a_{n}}(s, t, t, x) \\
& \leq \Phi 5.10(K)\left[a_{n}^{-2 \varepsilon_{0}}+2^{4 N}[5.10]\left\{|t-s|^{1-\eta_{1} / 4}\left[\left((t-s) \vee a_{n}\right)^{\gamma \tilde{\gamma}_{m}-3 / 2}+a_{n}^{2 \beta \gamma}\left((t-s) \vee a_{n}\right)^{\gamma-3 / 2}\right]\right.\right. \\
& \left.+1\left(a_{n}<2^{-2 N}\right)\left((t-s) \wedge a_{n}\right) a_{n}^{-3 / 2} 2^{N \eta_{1} / 2}\left[2^{-2 N \gamma \tilde{\gamma}_{m}}+a_{n}^{2 \beta \gamma} 2^{-2 N \gamma}\right]\right\} \\
& \text { for all } s \leq t
\end{aligned}
$$

Moreover $N_{5.10}$ is stochastically bounded uniformly in $(n, \beta)$. 
Proof. Let $\xi=1-(4 \gamma R)^{-1}$ and define $N_{5.10}=N_{1}\left(m, n, \xi(R), \varepsilon_{0}, K, \beta\right)$ so that the last statement is immediate from $\left(P_{m}\right)$. We may assume $t \geq a_{n}$. By Lemma 5.2 (again the maximum with $\sqrt{a_{n}}$ may be ignored in the calculation below) and then Lemma 4.2, we get for $\omega$ as in (5.45) and $s \leq t$,

$$
\begin{aligned}
& Q_{T, a_{n}}(s, t, t, x) \\
& \leq 95.2 \int_{\left(s-a_{n}\right)^{+}}^{t-a_{n}} \int p_{t-r}^{\prime}(y-x)^{2} e^{2 R_{1} K} e^{2\left(R_{1}+1\right)|y-x|}\left[2^{-N}+\sqrt{t-r}+|y-x|\right]^{2 \gamma \xi} \\
& \times\left[\left(2^{-N}+\sqrt{t-r}+|y-x|\right)^{\tilde{\gamma}_{m}-1}+a_{n}^{\beta}\right]^{2 \gamma} d y d r \\
& \leq c_{1}(K) C\left[5.2 \int_{\left(s-a_{n}\right)^{+}}^{t-a_{n}}(t-r)^{-1} \int p_{2(t-r)}(z)^{2} e^{2\left(R_{1}+1\right)|z|}\left[2^{-2 N \gamma \xi}+(t-r)^{\gamma \xi}+|z|^{2 \gamma \xi}\right]\right. \\
& \times\left[\left(2^{-2 N \gamma\left(\tilde{\gamma}_{m}-1\right)}+(t-r)^{\gamma\left(\tilde{\gamma}_{m}-1\right)}+|z|^{2 \gamma\left(\tilde{\gamma}_{m}-1\right)}+a_{n}^{2 \beta \gamma}\right] d z d r\right. \\
& \leq c_{2}(K) C_{5.2} \int_{\left(s-a_{n}\right)^{+}}^{t-a_{n}}(t-r)^{-3 / 2}\left[2^{-2 N \gamma \xi}+(t-r)^{\gamma \xi}\right] \\
& \times\left[\left(2^{-2 N \gamma\left(\tilde{\gamma}_{m}-1\right)}+(t-r)^{\gamma\left(\tilde{\gamma}_{m}-1\right)}\right)+a_{n}^{2 \beta \gamma}\right] d r \\
& \leq c_{3}(K) C_{5.2}\left[\int_{\left(s-a_{n}\right)^{+}}^{t-a_{n}} 1\left(r<t-2^{-2 N}\right)\left[(t-r)^{\gamma\left(\tilde{\gamma}_{m}+\xi-1\right)-3 / 2}+a_{n}^{2 \beta \gamma}(t-r)^{\gamma \xi-3 / 2}\right] d r\right. \\
& \left.+\int_{\left(s-a_{n}\right)^{+}}^{t-a_{n}} 1\left(r \geq t-2^{-2 N}\right)(t-r)^{-3 / 2} d r 2^{-2 N \gamma \xi}\left[2^{-2 N \gamma\left(\tilde{\gamma}_{m}-1\right)}+a_{n}^{2 \beta \gamma}\right]\right] \\
& \equiv c_{3}(K) C_{5.2}\left[J_{1}+J_{2}\right] \text {. }
\end{aligned}
$$

As in the derivation of (5.20), now with $\bar{d}_{N}=2^{-N}, \delta=a_{n}$ and $s^{\prime}=t$, we get

$$
J_{2} \leq 1\left(a_{n}<2^{-2 N}\right) 2\left(a_{n} \wedge(t-s)\right) a_{n}^{-3 / 2} 2^{2 N \gamma(1-\xi)}\left[2^{-2 N \gamma \tilde{\gamma}_{m}}+a_{n}^{2 \beta \gamma} 2^{-2 N \gamma}\right] .
$$

For $J_{1}$, let $p=\gamma\left(\tilde{\gamma}_{m}+\xi-1\right)-3 / 2$ or $p=\gamma \xi-3 / 2$. Our choice of $\xi$ and $R$ implies $p \in[-15 / 16,1 / 2]$ and so, considering $p \geq 0$ and $p<0$ separately, we arrive at

$$
\begin{aligned}
\int_{\left(s-a_{n}\right)^{+}}^{t-a_{n}}(t-r)^{p} d r \leq 16\left[\left(t-s+a_{n}\right)^{p+1}-a_{n}^{p+1}\right] & \leq 16\left(2^{p^{+}}\right)(t-s)\left((t-s) \vee a_{n}\right)^{p} \\
& \leq 24(t-s)\left((t-s) \vee a_{n}\right)^{p}
\end{aligned}
$$

Therefore

$$
\begin{aligned}
J_{1} & \leq 24(t-s)\left[\left((t-s) \vee a_{n}\right)^{\gamma\left(\tilde{\gamma}_{m}+\xi-1\right)-3 / 2}+a_{n}^{2 \beta \gamma}\left((t-s) \vee a_{n}\right)^{\gamma \xi-3 / 2}\right] \\
& \leq 24(t-s)^{1-\gamma(1-\xi)}\left[\left((t-s) \vee a_{n}\right)^{\gamma \tilde{\gamma}_{m}-3 / 2}+a_{n}^{2 \beta \gamma}\left((t-s) \vee a_{n}\right)^{\gamma-3 / 2}\right] .
\end{aligned}
$$

Put (5.47) and (5.48) into (5.46), noting that $\gamma(1-\xi(R))=(4 R)^{-1}<\eta_{1} / 4$ and $t-s \leq K$, to complete the proof. 
Proposition 5.11 Let $0 \leq m \leq \bar{m}+1$ and assume $\left(P_{m}\right)$. For any $n \in \mathbb{N}, \eta_{1} \in(0,1 / 2), \varepsilon_{0} \in(0,1)$, $K \in \mathbb{N}^{\geq K_{1}}$, and $\beta \in[0,1 / 2]$, there is an $N_{\overline{5.11}}=N_{\overline{5.11}}\left(m, n, \eta_{1}, \varepsilon_{0}, K, \beta\right)(\omega) \in \mathbb{N}$ a.s. such that for all $N \geq N_{5.11},(t, x) \in Z(N, n, K, \beta), s \leq t$ and $\sqrt{t-s} \leq N^{-4 / \eta_{1}}$ implies that

$$
\begin{aligned}
& \left|F_{a_{n}}(s, t, x)-F_{a_{n}}(t, t, x)\right| \\
& \leq 2^{-81} a_{n}^{-\varepsilon_{0}}\left\{2^{-N\left(1-\eta_{1}\right)}\left(a_{n}^{1 / 2} \vee 2^{-N}\right)^{\left(\gamma_{m+1}-2\right) \wedge 0}\right. \\
& \quad+2^{N \eta_{1}} a_{n}^{-1 / 4}\left(\frac{2^{-N}}{\sqrt{a_{n}}}+1\right)\left(2^{-N \gamma \tilde{\gamma}_{m}}+a_{n}^{\beta \gamma}\left(\sqrt{a_{n}} \vee 2^{-N}\right)^{\gamma}\right) \\
& \left.\quad+(t-s)^{\left(1-\eta_{1}\right) / 2}\left(\left(\sqrt{t-s} \vee \sqrt{a_{n}}\right)^{\gamma \tilde{\gamma}_{m}-\frac{3}{2}}+a_{n}^{\beta \gamma}\left(\sqrt{t-s} \vee \sqrt{a_{n}}\right)^{\gamma-\frac{3}{2}}\right)\right\} .
\end{aligned}
$$

Moreover $N_{5.11}$ is stochastically bounded, uniformly in $(n, \beta)$.

Proof. Apply Lemma 5.10 with $R=2 / \eta_{1}$ so that on

$$
\left\{\omega:(t, x) \in Z(N, n, K, \beta), N \geq N\left[\overline{5.10}\left(m, n, 2 / \eta_{1}, \varepsilon_{0}, K, \beta\right)\right\}\right.
$$

for $s \leq t$,

$$
\begin{aligned}
& R_{0}^{\gamma} Q_{T, a_{n}}(s, t, t, x)^{1 / 2} \\
& \leq c_{1}(K) R_{0}^{\gamma}\left[a_{n}^{-\varepsilon_{0}}+2^{2 N[5.10]}\left\{( \sqrt { t - s } ) ^ { \eta _ { 1 } / 4 } ( \sqrt { t - s } ) ^ { 1 - \frac { \eta _ { 1 } } { 2 } } \left[\left(\sqrt{t-s} \vee \sqrt{a_{n}}\right)^{\gamma \tilde{\gamma} m-\frac{3}{2}}\right.\right.\right. \\
& \left.+a_{n}^{\beta \gamma}\left(\sqrt{t-s} \vee \sqrt{a_{n}}\right)^{\gamma-\frac{3}{2}}\right] \\
& \left.+2^{-N \eta_{1} / 4} a_{n}^{-1 / 4} 2^{N \eta_{1} / 2}\left[2^{-N \gamma \tilde{\gamma}_{m}}+a_{n}^{\beta \gamma} 2^{-N \gamma}\right]\right\} .
\end{aligned}
$$

Let $N_{2}\left(m, n, \eta_{1}, \varepsilon_{0}, K, \beta\right)(\omega)=\frac{8}{\eta_{1}}\left[N_{5.10}+N_{0}(K)\right]$, where $N_{0}(K) \in \mathbb{N}$ is chosen large enough so that

$$
\begin{aligned}
c_{1}(K) R_{0}^{\gamma}\left[a_{n}^{-\varepsilon_{0}}+2^{2 N[5.10}\right] 2^{-\frac{\eta_{1}}{4} N_{2}} & \leq c_{1}(K) R_{0}^{\gamma}\left[a_{n}^{-\varepsilon_{0}}+2^{2 N[5.10]} 2^{-2 N}\left[5.10{ }^{-2 N_{0}(K)}\right.\right. \\
& \leq 2^{-100} a_{n}^{-\varepsilon_{0}} .
\end{aligned}
$$

It follows from (5.49) and (5.50) that for $N \geq N_{2},(t, x) \in Z(N, n, K, \beta), s \leq t$, and $\sqrt{t-s} \leq 2^{-N_{2}}$,

$$
\begin{aligned}
& R_{0}^{\gamma} Q_{T, a_{n}}(s, t, t, x)^{1 / 2} \\
& \leq 2^{-100} a_{n}^{-\varepsilon_{0}}\left\{(\sqrt{t-s})^{1-\frac{\eta_{1}}{2}}\left[\left(\sqrt{t-s} \vee \sqrt{a_{n}}\right)^{\gamma \tilde{\gamma}_{m}-\frac{3}{2}}+a_{n}^{\beta \gamma}\left(\sqrt{t-s} \vee \sqrt{a_{n}}\right)^{\gamma-\frac{3}{2}}\right]\right. \\
& \left.\quad+a_{n}^{-1 / 4} 2^{N \eta_{1} / 2}\left[2^{-N \gamma \tilde{\gamma}_{m}}+a_{n}^{\beta \gamma} 2^{-N \gamma}\right]\right\} \\
& \equiv(\sqrt{t-s})^{1-\frac{\eta_{1}}{2}} \Delta_{1}\left(m, n, \sqrt{t-s} \vee \sqrt{a_{n}}\right)+2^{N \eta_{1} / 2} \Delta_{2}\left(m, n, 2^{-N}\right) .
\end{aligned}
$$


Combine this with (3.2), (15.6) (now with $t^{\prime}=t=s^{\prime}, x=x^{\prime}$, so the second integral there is 0) and the Dubins-Schwarz theorem to see that if $B(\cdot)$ is a standard 1-dimensional Brownian motion, then

$$
\begin{aligned}
& P\left(\left|F_{a_{n}}(s, t, x)-F_{a_{n}}(t, t, x)\right| \geq(\sqrt{t-s})^{1-\eta_{1}} \Delta_{1}\left(m, n, \sqrt{t-s} \vee \sqrt{a_{n}}\right)+2^{N \eta_{1}} \Delta_{2}\left(m, n, 2^{-N}\right),\right. \\
& \left.\quad(t, x) \in Z(N, n, K, \beta), N \geq N_{2}, \sqrt{t-s} \leq 2^{-N_{2}}\right) \\
& \leq P\left(\sup _{u \leq 1}|B(u)| \geq(\sqrt{t-s})^{-\eta_{1} / 2} \wedge 2^{N \eta_{1} / 2}\right) 1(t-s \leq 1) \\
& \leq c_{0} \exp \left\{-\frac{1}{2}\left[(t-s)^{-\eta_{1} / 2} \wedge 2^{N \eta_{1}}\right]\right\} .
\end{aligned}
$$

Let $\ell_{N}=2^{2(N+3)} N^{-8 / \eta_{1}}$ and set

$$
\begin{array}{r}
M_{N}(\omega)=\max \left\{\frac{\left|F_{a_{n}}\left(i 2^{-2(N+2)}, j 2^{-2(N+2)}, k 2^{-(N+2)}\right)-F_{a_{n}}\left(j 2^{-2(N+2)}, j 2^{-2(N+2)}, k 2^{-(N+2)}\right)\right|}{\left(\sqrt{j-i} 2^{-(N+2)}\right)^{1-\eta_{1}} \Delta_{1}\left(m, n,\left(\sqrt{j-i} 2^{-(N+2)}\right) \vee \sqrt{a_{n}}\right)+2^{N \eta_{1}} \Delta_{2}\left(m, n, 2^{-N}\right)}:\right. \\
\left.0 \leq j-i \leq \ell_{N},\left(j 2^{-2(N+2)}, k 2^{-(N+2)}\right) \in Z(N, n, K, \beta), i, j \in \mathbb{Z}_{+}, k \in \mathbb{Z}\right\} .
\end{array}
$$

If $N_{3}=2^{N_{2}}$, then

$$
N \geq N_{3} \Rightarrow N^{-4 / \eta_{1}} \leq 2^{-N_{2}-1} \Rightarrow \sqrt{\ell_{N}} 2^{-N-2}=2 N^{-4 / \eta_{1}} \leq 2^{-N_{2}} .
$$

The fact that $M_{N}=0$ if $\ell_{N}<1$, (5.51), and (5.52) imply

$$
\begin{aligned}
& P\left(M_{N} \geq 1, N \geq N_{3}\right) \\
& \leq(K+1)^{2} 2^{4(N+2)}(2 K+1) 2^{N+2} c_{0} \exp \left\{-\frac{1}{2}\left(\left(\ell_{N} 2^{-2(N+2)}\right)^{-\eta_{1} / 2} \wedge 2^{N \eta_{1}}\right)\right\} 1\left(\ell_{N} \geq 1\right) \\
& \leq c_{1} K^{3} 2^{5 N} \exp \left\{-\frac{1}{2}\left(\left(\sqrt{\ell_{N}} 2^{-N}\right) \vee 2^{-N}\right)^{-\eta_{1}}\right\} 1\left(\ell_{N} \geq 1\right) \\
& \leq c_{1} K^{3} 2^{5 N} \exp \left\{-2^{-5 / 2} N^{4}\right\} \quad\left(\text { recall } \eta_{1}<1 / 2\right) .
\end{aligned}
$$

If $A_{N}=\left\{M_{N} \geq 1, N \geq N_{3}\right\}$ and

$$
N_{4}=N_{4}\left(m, n, \eta_{1}, \varepsilon_{0}, K, \beta\right)(\omega)=\min \left\{N: \omega \in \cap_{N^{\prime}=N}^{\infty} A_{N^{\prime}}^{c}\right\},
$$

then

$$
\begin{aligned}
P\left(N_{4}>N\right)=P\left(\cup_{N^{\prime}=N}^{\infty} A_{N^{\prime}}\right) & \leq c_{1} K^{3} \sum_{N^{\prime}=N}^{\infty} 2^{5 N^{\prime}} \exp \left\{-2^{-5 / 2}\left(N^{\prime}\right)^{4}\right\} \\
& \leq c_{2}(K) \exp \left(-N^{4} / 6\right)
\end{aligned}
$$

Let $N_{5}\left(\eta_{1}\right)$ be large enough so that

$$
N \geq N_{5} \Rightarrow 2^{1-N} \leq N^{-4 / \eta_{1}}
$$

Define

$$
N_{5.11}\left(m, n, \eta_{1}, \varepsilon_{0}, K, \beta\right)=\left(N_{5.8} \vee N_{3} \vee N_{4} \vee N_{5}\right)+2
$$


It follows from Proposition 5.8, (5.53), and the definition of $N_{3}$ that $N_{5.11}$ is stochastically bounded uniformly in $(n, \beta)$. Assume

$$
N \geq N 5.11,(t, x) \in Z(N, n, K, \beta), s \leq t \text { and } \sqrt{t-s} \leq N^{-4 / \eta_{1}} .
$$

Case 1. $\sqrt{t-s} \geq 2^{1-N}$.

The condition $N \geq N_{5.11}$ implies $\omega \in A_{N-2}^{c}$ and $N-2 \geq N_{3}$, which in turn implies

$$
M_{N-2}<1
$$

Let $s_{\ell}=\left\lfloor 2^{2 \ell} s\right\rfloor 2^{-2 \ell}, t_{\ell}=\left\lfloor 2^{2 \ell} t\right\rfloor 2^{-2 \ell}$ and $x_{\ell}=\operatorname{sgn}(x)\left\lfloor 2^{\ell}|x|\right\rfloor 2^{-\ell}$, be the usual dyadic approximations to $s, t$ and $x$, respectively, and let $\left(\hat{t}_{0}, \hat{x}_{0}\right)$ be as in the definition of $(t, x) \in Z(N, n, K, \beta)$. Then

$$
d\left(\left(t_{N}, x_{N}\right),\left(\hat{t}_{0}, \hat{x}_{0}\right)\right) \leq 2^{-N}+\sqrt{t-t_{N}}+\left|x-x_{N}\right| \leq 2^{2-N}, t_{N} \leq t \leq T_{K},\left|x_{N}\right| \leq|x| \leq K,
$$

and so

$$
\left(t_{N}, x_{N}\right) \in Z(N-2, n, K, \beta)
$$

Write

$$
\begin{aligned}
\left|F_{a_{n}}(s, t, x)-F_{a_{n}}(t, t, x)\right| \leq & {\left[\left|F_{a_{n}}(s, t, x)-F_{a_{n}}\left(s_{N}, t_{N}, x_{N}\right)\right|+\left|F_{a_{n}}(t, t, x)-F_{a_{n}}\left(t_{N}, t_{N}, x_{N}\right)\right|\right] } \\
& +\left[\left|F_{a_{n}}\left(s_{N}, t_{N}, x_{N}\right)-F_{a_{n}}\left(t_{N}, t_{N}, x_{N}\right)\right|\right] \\
\equiv & T_{1}+T_{2} .
\end{aligned}
$$

The fact that $(t, x) \in Z(N, n, K, \beta), t_{N} \leq t \leq T_{K}, s \leq t \leq K$,

$$
d\left((t, t, x),\left(t_{N}, t_{N}, x_{N}\right)\right) \vee d\left((s, t, x),\left(s_{N}, t_{N}, x_{N}\right)\right) \leq 3\left(2^{-N}\right) \leq 2^{-(N-2)},
$$

and $N-2 \geq N_{5.8}$, allows us to use Proposition 5.8 and infer that

$$
T_{1} \leq 2^{-85} 2^{-(N-2)\left(1-\eta_{1}\right)} \bar{\Delta}_{u_{1}^{\prime}}\left(m, n, 1, \varepsilon_{0}, 2^{-(N-2)}\right) .
$$

For $T_{2}$ we have from $N \geq N_{5}$, (5.54), and the last part of (5.55),

$$
\sqrt{t_{N}-s_{N}} \leq \sqrt{t-s}+2^{1-N} \leq 2 N^{-4 / \eta_{1}} \leq \sqrt{\ell_{N-2}} 2^{-N}
$$

In view of (5.57) and (5.56), this implies

$$
\begin{aligned}
T_{2} & \leq M_{N-2}\left[{\sqrt{t_{N}-s_{N}}}^{1-\eta_{1}} \Delta_{1}\left(m, n, \sqrt{t_{N}-s_{N}} \vee \sqrt{a_{n}}\right)+2^{(N-2) \eta_{1}} \Delta_{2}\left(m, n, 2^{-(N-2)}\right)\right] \\
& \leq\left[{\sqrt{t_{N}-s_{N}}}^{1-\eta_{1}} \Delta_{1}\left(m, n, \sqrt{t_{N}-s_{N}} \vee \sqrt{a_{n}}\right)+2^{(N-2) \eta_{1}} \Delta_{2}\left(m, n, 2^{-(N-2)}\right)\right] .
\end{aligned}
$$

As $t-s \geq 2^{2-2 N}$ (recall this defines Case 1), we have

$$
t-s \leq\left(t-t_{N}\right)+\left(t_{N}-s_{N}\right) \leq 2^{-2 N}+\left(t_{N}-s_{N}\right) \leq \frac{1}{4}(t-s)+\left(t_{N}-s_{N}\right),
$$


and so

$$
t_{N}-s_{N} \geq \frac{1}{2}(t-s)
$$

More simply,

$$
t_{N}-s_{N} \leq t-s+2^{1-2 N} \leq 2(t-s) .
$$

Use (5.61) and (5.62) in (5.60) and then combine the result with (5.59) and (5.58) to conclude that

$$
\begin{aligned}
& \left|F_{a_{n}}(s, t, x)-F_{a_{n}}(t, t,, x)\right| \\
& \leq 2^{-85} 2^{-(N-2)\left(1-\eta_{1}\right)} a_{n}^{-\varepsilon_{0}}\left[\left(a_{n}^{1 / 2} \vee 2^{-(N-2)}\right)^{\left(\gamma_{m+1}-2\right) \wedge 0}\right. \\
& \left.+a_{n}^{-3 / 4}\left[2^{-(N-2) \gamma \tilde{\gamma}_{m}}+a_{n}^{\beta \gamma}\left(a_{n}^{1 / 2} \vee 2^{-(N-2)}\right)^{\gamma}\right]\right] \\
& +2^{-99} a_{n}^{-\varepsilon_{0}}(\sqrt{t-s})^{1-\eta_{1}}\left[\left(\sqrt{t-s} \vee \sqrt{a_{n}}\right)^{\gamma \tilde{\gamma}_{m}-\frac{3}{2}}+a_{n}^{\beta \gamma}\left(\sqrt{t-s} \vee \sqrt{a_{n}}\right)^{\gamma-\frac{3}{2}}\right] \\
& +2^{-100} a_{n}^{-\varepsilon_{0}-\frac{1}{4}} 2^{(N-2) \eta_{1}}\left[2^{-(N-2) \gamma \tilde{\gamma}_{m}}+a_{n}^{\beta \gamma} 2^{-(N-2) \gamma}\right] .
\end{aligned}
$$

Next use

$$
2^{-N\left(1-\eta_{1}\right)} a_{n}^{-3 / 4}+2^{N \eta_{1}} a_{n}^{-1 / 4}=a_{n}^{-1 / 4} 2^{N \eta_{1}}\left[\frac{2^{-N}}{\sqrt{a_{n}}}+1\right]
$$

to combine the first and third terms in (5.63) and conclude, after a bit of arithmetic, that

$$
\begin{aligned}
& \left|F_{a_{n}}(s, t, x)-F_{a_{n}}(t, t, x)\right| \\
& \leq 2^{-81} a_{n}^{-\varepsilon_{0}}\left\{\left[a_{n}^{-1 / 4} 2^{N \eta_{1}}\left(\frac{2^{-N}}{\sqrt{a_{n}}}+1\right)\left(2^{-N \gamma \tilde{\gamma}_{m}}+a_{n}^{\beta \gamma}\left(a_{n}^{1 / 2} \vee 2^{-N}\right)^{\gamma}\right)\right.\right. \\
& \left.\quad+2^{-N\left(1-\eta_{1}\right)}\left(a_{n}^{1 / 2} \vee 2^{-N}\right)^{\left(\gamma_{m+1}-2\right) \wedge 0}\right] \\
& \left.+(\sqrt{t-s})^{1-\eta_{1}}\left(\left(\sqrt{t-s} \vee \sqrt{a_{n}}\right)^{\gamma \tilde{\gamma}_{m}-\frac{3}{2}}+a_{n}^{\beta \gamma}\left(\sqrt{t-s} \vee \sqrt{a_{n}}\right)^{\gamma-\frac{3}{2}}\right)\right\} .
\end{aligned}
$$

Case 2. $\sqrt{t-s} \leq 2^{1-N}$.

As $(t, x) \in Z(N-1, n, K, \beta)$ (by (5.55)), $s \leq t \leq K, N-1 \geq N$ [5.8, and $d((s, t, x),(t, t, x)) \leq$ $2^{-(N-1)}$, we may use Proposition 5.8 with $\alpha=1$ to conclude

$$
\begin{aligned}
& \left|F_{a_{n}}(s, t, x)-F_{a_{n}}(t, t, x)\right| \\
& \leq 2^{-86}(\sqrt{t-s})^{1-\eta_{1}} \bar{\Delta}_{u_{1}^{\prime}}\left(m, n, 1, \varepsilon_{0}, 2^{-(N-1)}\right) \\
& \leq 2^{-83} 2^{-N\left(1-\eta_{1}\right)} a_{n}^{-\varepsilon_{0}}\left[a_{n}^{-3 / 4} 2^{-N \gamma \tilde{\gamma}_{m}}+\left(a_{n}^{1 / 2} \vee 2^{-N}\right)^{\left(\gamma_{m+1}-2\right) \wedge 0}+a_{n}^{-3 / 4+\beta \gamma}\left(a_{n}^{1 / 2} \vee 2^{-N}\right)^{\gamma}\right] \\
& \leq 2^{-83} a_{n}^{-\varepsilon_{0}}\left\{a_{n}^{-1 / 4} 2^{N \eta_{1}}\left(\frac{2^{-N}}{\sqrt{a_{n}}}\right)\left(2^{-N \gamma \tilde{\gamma}_{m}}+a_{n}^{\beta \gamma}\left(a_{n}^{1 / 2} \vee 2^{-N}\right)^{\gamma}\right)+2^{-N\left(1-\eta_{1}\right)}\left(a_{n}^{1 / 2} \vee 2^{-N}\right)^{\left(\gamma_{m+1}-2\right) \wedge 0}\right\}
\end{aligned}
$$

which is bounded by the first term on the right-hand side of (5.64).

We also need an analogue of Proposition 5.8 for $G_{a_{n}^{\alpha}}$. A subset of the arguments in Lemma 3.1 shows that

$$
G_{\delta}(s, t, x)=\int_{0}^{(s-\delta)^{+}} \int p_{(t \vee s)-r}(y-x) D(r, y) W(d r, d y) \text { for all } s \text { a.s. for all }(t, x),
$$


which is just the analogue of the expression for $F_{\delta}$, (3.6), with $p_{t-r}$ in place of $p_{t-r}^{\prime}$. Although we only will need bounds on $G_{a_{n}^{\alpha}}(s, t, x)-G_{a_{n}^{\alpha}}(t, t, x)$ (and for $\sqrt{t-s}$ small as in Proposition [5.8), this seems to require bounds on the analogues of the three types of square functions handled in Lemmas 5.4, 5.5 and 5.6, but now with no derivatives on the Gaussian densities. This results in some simplification and a smaller singularity in $a_{n}^{\alpha}$. We omit the proof of the following result as the details are quite similar to those used to establish Proposition 5.8.

Proposition 5.12 Let $0 \leq m \leq \bar{m}+1$ and assume $\left(P_{m}\right)$. For any $n \in \mathbb{N}, \eta_{1} \in(0,1 / 2), \varepsilon_{0} \in(0,1)$, $K \in \mathbb{N}^{\geq K_{1}}, \alpha \in[0,1]$, and $\beta \in[0,1 / 2]$, there is an $N_{5.12}=\sqrt{5.12}\left(m, n, \eta_{1}, \varepsilon_{0}, K, \alpha, \beta\right) \in \mathbb{N}$ a.s. such that for all $N \geq N_{5.12},(t, x) \in Z(N, n, K, \beta), s \leq t$ and $\sqrt{t-s} \leq 2^{-N}$,

$$
\begin{aligned}
\left|G_{a_{n}^{\alpha}}(s, t, x)-G_{a_{n}^{\alpha}}(t, t, x)\right| \leq & 2^{-92}(t-s)^{\frac{1}{2}\left(1-\eta_{1}\right)} a_{n}^{-\varepsilon_{0}} a_{n}^{-\alpha / 4} \\
& \times\left[\left(a_{n}^{\alpha / 2} \vee 2^{-N}\right)^{\gamma \tilde{\gamma}_{m}}+a_{n}^{\beta \gamma}\left(a_{n}^{\alpha / 2} \vee 2^{-N}\right)^{\gamma}\right] .
\end{aligned}
$$

We need to use our global modulus of continuity for $u_{1, a_{n}^{\alpha}}^{\prime}$ (Corollary 5.9) to get a modulus for $u_{1, a_{n}^{\alpha}}$ itself. This is of course easy for spatial increments, but a key observation is that it is possible to also use control of the spatial derivatives to get a better modulus on the temporal increments.

Notation. Define

$$
\begin{aligned}
\bar{\Delta}_{u_{1}}\left(m, n, \alpha, \varepsilon_{0}, 2^{-N}\right)= & a_{n}^{-\varepsilon_{0}-3 \alpha / 4}\left[a_{n}^{\beta} a_{n}^{3 \alpha / 4}+a_{n}^{\beta \gamma}\left(a_{n}^{\alpha / 2} \vee 2^{-N}\right)^{\gamma+1}\right. \\
& \left.+\left(a_{n}^{\alpha / 2} \vee 2^{-N}\right)^{\gamma \tilde{\gamma}_{m}+1}+1(m \geq \bar{m}) a_{n}^{3 \alpha / 4}\left(a_{n}^{\alpha / 2} \vee 2^{-N}\right)\right] .
\end{aligned}
$$

Dependence on $\alpha$ or $\varepsilon_{0}$ is often suppressed.

If $\eta>0$ let $N \frac{1}{5.13}(\eta)$ be the smallest natural number such that $2^{1-N} \leq N^{-\frac{4}{\eta}}$ whenever $N \geq$ $N \frac{1}{5.13}(\eta)$.

Proposition 5.13 Let $0 \leq m \leq \bar{m}+1$ and assume $\left(P_{m}\right)$. For any $n \in \mathbb{N}, \eta_{1} \in(0,1 / 2), \varepsilon_{0}, \varepsilon_{1} \in$ $(0,1), K \in \mathbb{N}^{\geq K_{1}}, \alpha \in[0,1]$ and $\beta \in[0,1 / 2]$, there is an $N_{5.13}=N_{5.13}\left(m, n, \eta_{1}, \varepsilon_{0}, K, \alpha, \beta\right) \in \mathbb{N}$ a.s. so that for all $N \geq N$ 5.13, $n, \alpha$ satisfying

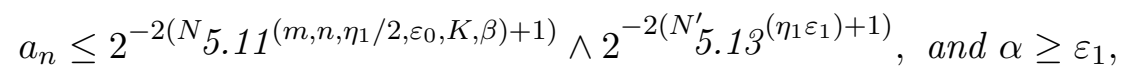

$(t, x) \in Z(N, n, K, \beta), t^{\prime} \leq T_{K}$, if $d\left((t, x),\left(t^{\prime}, x^{\prime}\right)\right) \leq 2^{-N}$, then

$$
\left|u_{1, a_{n}^{\alpha}}(t, x)-u_{1, a_{n}^{\alpha}}\left(t^{\prime}, x^{\prime}\right)\right| \leq 2^{-90} d\left((t, x),\left(t^{\prime}, x^{\prime}\right)\right)^{1-\eta_{1}} \bar{\Delta}_{u_{1}}\left(m, n, \alpha, \varepsilon_{0}, 2^{-N}\right) .
$$

Moreover $N_{5.13}$ is stochastically bounded uniformly in $n \in \mathbb{N}, \alpha \in[0,1]$ and $\beta \in[0,1 / 2]$.

Remark. Although $n$ appears on both sides of (5.67), the stochastic boundedness of $N_{5.11}$ ensures it will hold for infinitely many $n$. This condition becomes stronger as $\alpha$ goes to 0 and $a_{n}^{\alpha}$ moves away from the value $a_{n}$ where the definition of $Z(N, n, K, \beta)$ ensures some control on $u_{1, a_{n}}^{\prime}$. This effectively rules out $\alpha=0$ from the above conclusion. 
Proof. Let

$N_{5.13}^{\prime \prime}\left(m, n, \eta_{1}, \varepsilon_{0}, K, \alpha, \beta\right)=\left(\left(2 N[5.8)\left(m, n, \eta_{1} / 2, \varepsilon_{0}, K+1, \alpha, \beta\right) \vee N_{5.12}\left(m, n, \eta_{1}, \varepsilon_{0}, K+1, \alpha, \beta\right)\right)+1\right.$.

Clearly $N \frac{11}{5.13}$ is stochastically bounded uniformly in $(n, \alpha, \beta)$. Assume (5.67) and

$$
N \geq N \text { If.13, }(t, x) \in Z(N, n, K, \beta), t^{\prime} \leq T_{K} \text { and } d\left((t, x),\left(t^{\prime}, x^{\prime}\right)\right) \leq 2^{-N} .
$$

As in the proof of Proposition [5.8, $\left(t^{\prime}, x^{\prime}\right) \in Z(N-1, n, K+1, \beta)$, and by interchanging $(t, x)$ with $\left(t^{\prime}, x^{\prime}\right), N$ with $N-1$ and $K$ with $K+1$, in the argument below (again as in the proof of Proposition 5.8) we may assume without loss of generality that $t^{\prime} \leq t$. Indeed, this is the reason for having $K+1$ and adding 1 in our definition of $N \frac{\prime \prime}{5.13}$.

Recall that

$$
G_{a_{n}^{\alpha}}\left(t^{\prime}, t, x\right)=P_{t-t^{\prime}+a_{n}^{\alpha}}\left(u\left(\left(t^{\prime}-a_{n}^{\alpha}, \cdot\right)\right)(x)=P_{t-t^{\prime}}\left(u_{1, a_{n}^{\alpha}}\left(t^{\prime}, \cdot\right)\right)(x),\right.
$$

and so

$$
\begin{aligned}
\left|u_{1, a_{n}^{\alpha}}\left(t^{\prime}, x^{\prime}\right)-u_{1, a_{n}^{\alpha}}(t, x)\right| \leq & \left|u_{1, a_{n}^{\alpha}}\left(t^{\prime}, x^{\prime}\right)-u_{1, a_{n}^{\alpha}}\left(t^{\prime}, x\right)\right|+\left|u_{1, a_{n}^{\alpha}}\left(t^{\prime}, x\right)-P_{t-t^{\prime}}\left(u_{1, a_{n}^{\alpha}}\left(t^{\prime}, \cdot\right)\right)(x)\right| \\
& +\left|G_{a_{n}^{\alpha}}\left(t^{\prime}, t, x\right)-G_{a_{n}^{\alpha}}(t, t, x)\right| \\
\equiv & T_{1}+T_{2}+T_{3} .
\end{aligned}
$$

For $T_{1}$, let $\left(\hat{t}_{0}, \hat{x}_{0}\right)$ be as in the definition of $(t, x) \in Z(N, n, K, \beta)$. For $y$ between $x$ and $x^{\prime}$, $d\left(\left(t^{\prime}, y\right),(t, x)\right) \leq 2^{-N}$, and also $d\left(\left(\hat{t}_{0}, \hat{x}_{0}\right),(t, x)\right) \leq 2^{-N}$. Therefore by Corollary 5.9 (twice) with $\eta_{1} / 2$ in place of $\eta_{1}$,

$$
\begin{aligned}
&\left|u_{1, a_{n}^{\alpha}}^{\prime}\left(t^{\prime}, y\right)\right| \leq \mid u_{1, a_{n}^{\alpha}}^{\prime}\left(t^{\prime}, y\right)-u_{1, a_{n}^{\alpha}}^{\prime}(t, x)|+| u_{1, a_{n}^{\alpha}}^{\prime}(t, x)-u_{1, a_{n}^{\alpha}}^{\prime}\left(\hat{t}_{0}, \hat{x}_{0}\right) \mid \\
&+\left|u_{1, a_{n}^{\alpha}}^{\prime}\left(\hat{t}_{0}, \hat{x}_{0}\right)-u_{1, a_{n}}^{\prime}\left(\hat{t}_{0}, \hat{x}_{0}\right)\right|+a_{n}^{\beta} \\
&\left.\leq 2^{-84} 2^{-N(1-} \frac{\eta_{1}}{2}\right) \bar{\Delta}_{u_{1}^{\prime}}\left(m, n, \alpha, \varepsilon_{0}, 2^{-N}\right) \\
&+\left|F_{a_{n}}\left(\hat{t}_{0}-a_{n}^{\alpha}+a_{n}, \hat{t}_{0}, \hat{x}_{0}\right)-F_{a_{n}}\left(\hat{t}_{0}, \hat{t}_{0}, \hat{x}_{0}\right)\right|+a_{n}^{\beta} .
\end{aligned}
$$

We have used (5.44) in the last line.

We now use Proposition 5.11 to control the $F$ increment in (5.71). Choose $N^{\prime}$ so that

$$
2^{-N^{\prime}-1} \leq \sqrt{a_{n}} \leq 2^{-N^{\prime}}
$$

(5.67) implies $\sqrt{a_{n}} \leq 2^{-N} \overline{5.11}^{\left(m, n, \frac{\eta}{1}^{2}, \varepsilon_{0}, K, \beta\right)-1}$ and so

$$
N^{\prime} \geq N_{5.11}\left(m, n, \eta_{1} / 2, \varepsilon_{0}, K, \beta\right) .
$$

In addition, (5.67) implies $2^{-N^{\prime}-1} \leq \sqrt{a_{n}} \leq 2^{-N{ }^{\prime}} \underline{5.13}^{\left(\eta_{1} \varepsilon_{1}\right)-1}$ and so $N^{\prime} \geq N \frac{N^{\prime}}{5.13}$ which in turn implies

$$
a_{n}^{\alpha / 2} \leq 2^{-N^{\prime} \alpha} \leq 2^{-N^{\prime} \varepsilon_{1}} \leq N^{\prime-\frac{4 \varepsilon_{1}}{\eta_{1} \varepsilon_{1}}}=N^{\prime-\frac{4}{\eta_{1}}}
$$

Since

$$
\left|u\left(\hat{t}_{0}, \hat{x}_{0}\right)\right| \leq a_{n}=a_{n} \wedge\left(\sqrt{a_{n}} 2^{-N^{\prime}}\right) \quad(\text { by }(\underline{5.72}))
$$


we see that $\left(\hat{t}_{0}, \hat{x}_{0}\right) \in Z\left(N^{\prime}, n, K, \beta\right)$. (5.73) and (5.74) allow us to apply Proposition 5.11 with $N^{\prime}$ in place of $N,\left(\hat{t}_{0}, \hat{x}_{0}\right)$ in place of $(t, x), \eta_{1} / 2$ in place of $\eta_{1}$, and $s=\hat{t}_{0}-a_{n}^{\alpha}+a_{n}$, and deduce

$$
\begin{aligned}
& \left|F_{a_{n}}\left(\hat{t}_{0}-a_{n}^{\alpha}+a_{n}, \hat{t}_{0}, \hat{x}_{0}\right)-F_{a_{n}}\left(\hat{t}_{0}, \hat{t}_{0}, \hat{x}_{0}\right)\right| \\
& \leq 2^{-78} a_{n}^{-\varepsilon_{0}}\left[{\sqrt{a_{n}}}^{\left(1-\frac{\eta_{1}}{2}\right)}{\sqrt{a_{n}}}^{\left(\gamma_{m+1}-2\right) \wedge 0}+{\sqrt{a_{n}}}^{-\frac{\eta_{1}+1}{2}}\left(\sqrt{a_{n}} \gamma \tilde{\gamma}_{m}+a_{n}^{\beta \gamma} \sqrt{a_{n}} \gamma\right)\right. \\
& \left.+a_{n}^{\frac{\alpha}{2}\left(1-\frac{\eta_{1}}{2}\right)}\left(a_{n}^{\frac{\alpha}{2}\left(\gamma \tilde{\gamma}_{m}-\frac{3}{2}\right)}+a_{n}^{\beta \gamma} a_{n}^{\frac{\alpha}{2}\left(\gamma-\frac{3}{2}\right)}\right)\right] .
\end{aligned}
$$

The middle term in the square brackets is bounded by the last term because $\sqrt{a_{n}} \leq a_{n}^{\frac{\alpha}{2}}$. Therefore

$$
\begin{aligned}
& \left|F_{a_{n}}\left(\hat{t}_{0}-a_{n}^{\alpha}+a_{n}, \hat{t}_{0}, \hat{x}_{0}\right)-F_{a_{n}}\left(\hat{t}_{0}, \hat{t}_{0}, \hat{x}_{0}\right)\right| \\
& \leq 2^{-77} a_{n}^{-\varepsilon_{0}}\left[{\sqrt{a_{n}}}^{\left(1-\frac{\eta_{1}}{2}\right)}{\sqrt{a_{n}}}^{\left(\gamma_{m+1}-2\right) \wedge 0}+a_{n}^{\frac{\alpha}{2}\left(1-\frac{\eta_{1}}{2}\right)} a_{n}^{\frac{-3 \alpha}{4}}\left(a_{n}^{\frac{\alpha}{2} \gamma \tilde{\gamma}_{m}}+a_{n}^{\beta \gamma} a_{n}^{\frac{\alpha}{2} \gamma}\right)\right] .
\end{aligned}
$$

We also have

$$
{\sqrt{a_{n}}}^{1-\frac{\eta_{1}}{2}+\left(\gamma_{m+1}-2\right) \wedge 0} \leq\left(a_{n}^{\frac{\alpha}{2}} \vee 2^{-N}\right)^{1-\frac{\eta_{1}}{2}+\left(\gamma_{m+1}-2\right) \wedge 0},
$$

because the above exponent is positive since $\eta_{1}<1 / 2$ and $\gamma>3 / 4$. Use this bound in (5.75) and then insert the result into (5.71) to conclude that for any $y$ between $x$ and $x^{\prime}$,

$$
\begin{aligned}
& \left|u_{1, a_{n}^{\alpha}}^{\prime}\left(t^{\prime}, y\right)\right| \\
& \leq 2^{-77} a_{n}^{-\varepsilon_{0}}\left\{2^{-N\left(1-\frac{\eta_{1}}{2}\right)}\left(a_{n}^{\frac{\alpha}{2}} \vee 2^{-N}\right)^{\left(\gamma_{m+1}-2\right) \wedge 0}\right. \\
& +2^{-N\left(1-\frac{\eta_{1}}{2}\right)} a_{n}^{-\frac{3 \alpha}{4}}\left[2^{-N \gamma \tilde{\gamma}_{m}}+a_{n}^{\beta \gamma}\left(a_{n}^{\frac{\alpha}{2}} \vee 2^{-N}\right)^{\gamma}\right] \\
& +\left(a_{n}^{\frac{\alpha}{2}} \vee 2^{-N}\right)^{1-\frac{\eta_{1}}{2}+\left(\gamma_{m+1}-2\right) \wedge 0} \\
& \left.+a_{n}^{\frac{\alpha}{2}\left(1-\frac{\eta_{1}}{2}\right)} a_{n}^{\frac{-3 \alpha}{4}}\left(a_{n}^{\frac{\alpha}{2} \gamma \tilde{\gamma}_{m}}+a_{n}^{\beta \gamma} a_{n}^{\frac{\alpha}{2} \gamma}\right)\right\}+a_{n}^{\beta} \\
& \leq 2^{-76} a_{n}^{-\varepsilon_{0}}\left(a_{n}^{\frac{\alpha}{2}} \vee 2^{-N}\right)^{1-\frac{\eta_{1}}{2}}\left[\left(a_{n}^{\frac{\alpha}{2}} \vee 2^{-N}\right)^{\left(\gamma_{m+1}-2\right) \wedge 0}+a_{n}^{-\frac{3 \alpha}{4}}\left(a_{n}^{\frac{\alpha}{2}} \vee 2^{-N}\right)^{\gamma \tilde{\gamma}_{m}}\right. \\
& \left.+a_{n}^{-\frac{3 \alpha}{4}} a_{n}^{\beta \gamma}\left(a_{n}^{\frac{\alpha}{2}} \vee 2^{-N}\right)^{\gamma}\right]+a_{n}^{\beta} \\
& \equiv 2^{-76} \tilde{\Delta}_{u_{1}}\left(m, n, \alpha, \varepsilon_{0}, \eta_{1}, a_{n}^{\frac{\alpha}{2}} \vee 2^{-N}\right)+a_{n}^{\beta} \text {. }
\end{aligned}
$$

Note that $\tilde{\Delta}_{u_{1}}$ is monotone increasing in the $2^{-N} \vee a_{n}^{\frac{\alpha}{2}}$ variable due to the positivity of the exponents (since $\eta_{1}<1 / 2$ ). The Mean Value Theorem now shows that

$$
T_{1} \leq\left[a_{n}^{\beta}+2^{-76} \tilde{\Delta}_{u_{1}}\left(m, n, \alpha, \varepsilon_{0}, \eta_{1}, a_{n}^{\frac{\alpha}{2}} \vee 2^{-N}\right)\right]\left|x-x^{\prime}\right| .
$$

Recalling that $t^{\prime} \leq t$ and that (from (5.68) $) N \geq N$ 5.12 and $\sqrt{t-t^{\prime}} \leq 2^{-N}$, we may apply Proposition 5.12 and infer

$$
T_{3} \leq 2^{-92}\left(t-t^{\prime}\right)^{\frac{1}{2}\left(1-\eta_{1}\right)} a_{n}^{-\varepsilon_{0}-\alpha / 4}\left[\left(a_{n}^{\alpha / 2} \vee 2^{-N}\right)^{\gamma \tilde{\gamma}_{m}}+a_{n}^{\beta \gamma}\left(a_{n}^{\alpha / 2} \vee 2^{-N}\right)^{\gamma}\right] .
$$

For $T_{2}$, let $\{B(s): s \geq 0\}$ be a one-dimensional Brownian motion, starting at $x$ under $P_{x}$. Assume first that

$$
\left|B\left(t-t^{\prime}\right)-x\right| \leq 2^{-\frac{3}{2} N}[5.8 \text {. }
$$


Recalling (5.68) and that $N_{5.8} \geq 2$, we have

$$
\begin{aligned}
& d\left(\left(t^{\prime}, B\left(t-t^{\prime}\right)\right),(t, x)\right) \leq \sqrt{t-t^{\prime}}+2^{-\frac{3}{2} N}\left[5.8 \leq 2^{-N}+2^{-\frac{3}{2} N} \sqrt[5.8]{ }\right. \\
& \leq 2^{-2 N} 5.8+2^{-\frac{3}{2} N} 5.8 \\
& \leq 2^{-N}[5.8 \text {. }
\end{aligned}
$$

Define a random $N^{\prime} \in\left\{N_{5.8}, \ldots, N\right\}$ by

(i) if $d\left(\left(t^{\prime}, B\left(t-t^{\prime}\right)\right),(t, x)\right) \leq 2^{-N}$ then $N^{\prime}=N$;

(ii) if $d\left(\left(t^{\prime}, B\left(t-t^{\prime}\right)\right),(t, x)\right)>2^{-N}$ then $2^{-N^{\prime}-1}<d\left(\left(t^{\prime}, B\left(t-t^{\prime}\right)\right),(t, x)\right) \leq 2^{-N^{\prime}}$.

In case (ii) we have $2^{-N^{\prime}-1} \leq 2^{-N}+\left|B\left(t-t^{\prime}\right)-x\right|$, and so

$$
2^{-N^{\prime}} \leq 2^{1-N}+2\left|B\left(t-t^{\prime}\right)-x\right|,
$$

a result which is trivial in case (i). If $y$ is between $x$ and $B\left(t-t^{\prime}\right)$ we may argue as in (5.76), but now using $(t, x) \in Z\left(N^{\prime}, n, K, \beta\right)$, to see that

$$
\left|u_{1, a_{n}^{\alpha}}^{\prime}\left(t^{\prime}, y\right)\right| \leq 2^{-76} \tilde{\Delta}_{u_{1}}\left(m, n, \alpha, \varepsilon_{0}, \eta_{1}, a_{n}^{\frac{\alpha}{2}} \vee 2^{-N^{\prime}}\right)+a_{n}^{\beta} .
$$

Use (5.80) and the monotonicity of $\tilde{\Delta}_{u_{1}}$ observed above to see that

$$
\begin{aligned}
& a_{n}^{\varepsilon_{0}} \tilde{\Delta}_{u_{1}}\left(m, n, \alpha, \varepsilon_{0}, \eta_{1}, a_{n}^{\frac{\alpha}{2}} \vee 2^{-N^{\prime}}\right) \\
& \leq 8\left[\left[a_{n}^{\frac{\alpha}{2}}+2^{-N}+\left|B\left(t-t^{\prime}\right)-x\right|\right]^{1-\frac{\eta_{1}}{2}}\right. \\
& \quad \times\left\{\left(a_{n}^{\frac{\alpha}{2}}+2^{-N}+\left|B\left(t-t^{\prime}\right)-x\right|\right)^{\left(\gamma_{m+1}-2\right) \wedge 0}+a_{n}^{-\frac{3 \alpha}{4}}\left[\left(a_{n}^{\frac{\alpha}{2}}+2^{-N}+\left|B\left(t^{\prime}-t\right)-x\right|\right)^{\gamma \tilde{\gamma_{m}}}\right.\right. \\
& \left.\left.\left.\quad+a_{n}^{\beta \gamma}\left(a_{n}^{\frac{\alpha}{2}}+2^{-N}+\left|B\left(t^{\prime}-t\right)-x\right|\right)^{\gamma}\right]\right\}\right] .
\end{aligned}
$$

Use (5.82) in (5.81) and then the Mean Value Theorem to obtain (the expectation is over $B$ alone$N_{5.8}$ remains fixed-and we are dropping a number of small constants)

$$
\begin{aligned}
& E_{x}\left(1\left(\left|B\left(t-t^{\prime}\right)-x\right| \leq 2^{-\frac{3}{2} N} \underline{5.8}\right)\left|u_{1, a_{n}^{\alpha}}\left(t^{\prime}, B\left(t-t^{\prime}\right)\right)-u_{1, a_{n}^{\alpha}}\left(t^{\prime}, x\right)\right|\right) \\
& \leq E_{0}\left(| B ( t - t ^ { \prime } ) | \left\{a_{n}^{\beta}+a_{n}^{-\varepsilon_{0}}\left[a_{n}^{\frac{\alpha}{2}\left(1-\frac{\eta_{1}}{2}\right)}+2^{-N\left(1-\frac{\eta_{1}}{2}\right)}+\left|B\left(t-t^{\prime}\right)\right|^{1-\frac{\eta_{1}}{2}}\right]\left[a_{n}^{-3 \alpha / 4}\left[a_{n}^{\frac{\alpha}{2}}+2^{-N}+\left|B\left(t-t^{\prime}\right)\right|\right]^{\gamma \tilde{\gamma}_{m}}\right.\right.\right. \\
& \left.\left.+\left(a_{n}^{\alpha / 2} \vee 2^{-N}\right)^{\left(\gamma_{m+1}-2\right) \wedge 0}+a_{n}^{-3 \alpha / 4} a_{n}^{\beta \gamma}\left(a_{n}^{\alpha / 2}+2^{-N}+\left|B\left(t-t^{\prime}\right)\right|\right)^{\gamma}\right]\right\} \\
& \leq c_{1} \sqrt{t-t^{\prime}}\left\{a_{n}^{\beta}+a_{n}^{-\varepsilon_{0}}\left[\left(a_{n}^{\frac{\alpha}{2}} \vee 2^{-N}\right)^{\left(1-\frac{\eta_{1}}{2}\right)}+\left(t-t^{\prime}\right)^{\frac{1}{2}\left(1-\frac{\eta_{1}}{2}\right)}\right]\left[a_{n}^{-3 \alpha / 4}\left(a_{n}^{\frac{\alpha}{2}} \vee 2^{-N}+\sqrt{t-t^{\prime}}\right)^{\gamma \tilde{\gamma}_{m}}\right.\right. \\
& \left.+\left(a_{n}^{\alpha / 2} \vee 2^{-N}\right)^{\left(\gamma_{m+1}-2\right) \wedge 0}+a_{n}^{-3 \alpha / 4} a_{n}^{\beta \gamma}\left(a_{n}^{\alpha / 2} \vee 2^{-N}+\sqrt{t-t^{\prime}}\right)^{\gamma}\right\} \\
& \leq c_{2} \sqrt{t-t^{\prime}}\left\{a_{n}^{\beta}+a_{n}^{-\varepsilon_{0}}\left(a_{n}^{\frac{\alpha}{2}} \vee 2^{-N}\right)^{\left(1-\frac{\eta_{1}}{2}\right)}\left[\left(a_{n}^{\alpha / 2} \vee 2^{-N}\right)^{\left(\gamma_{m+1}-2\right) \wedge 0}+a_{n}^{-3 \alpha / 4}\left(a_{n}^{\alpha / 2} \vee 2^{-N}\right) \gamma \tilde{\gamma}_{m}\right.\right. \\
& \left.\left.+a_{n}^{-3 \alpha / 4} a_{n}^{\beta \gamma}\left(a_{n}^{\alpha / 2} \vee 2^{-N}\right)^{\gamma}\right]\right\} \quad\left(\text { since } \sqrt{t-t^{\prime}} \leq 2^{-N}\right) \\
& =c_{2} \sqrt{t-t^{\prime}}\left[a_{n}^{\beta}+\tilde{\Delta}_{u_{1}}\left(m, n, \alpha, \varepsilon_{0}, \eta_{1}, a_{n}^{\frac{\alpha}{2}} \vee 2^{-N}\right)\right] .
\end{aligned}
$$


To handle the complementary set to that on the left-hand side of (5.83), note that for $K \geq K_{1}$ and $t^{\prime} \leq T_{K}$,

$$
\left|u_{1, a_{n}^{\alpha}}\left(t^{\prime}, y\right)\right| \leq E_{y}\left(\left|u\left(\left(t^{\prime}-a_{n}^{\alpha}\right)^{+}, B\left(a_{n}^{\alpha}\right)\right)\right|\right) \leq 2 K E_{y}\left(e^{\left|B\left(a_{n}^{\alpha}\right)\right|}\right) \leq 2 K e^{1+|y|} .
$$

This and the fact that $\sqrt{t-t^{\prime}} \leq 2^{-2 N} \sqrt{5.8}$ imply that

$$
\begin{aligned}
& E_{x}\left(1\left(\left|B\left(t-t^{\prime}\right)-x\right|>2^{-\frac{3}{2} N} \sqrt[5.8]{ }\right)\left|u_{1, a_{n}^{\alpha}}\left(t^{\prime}, B\left(t-t^{\prime}\right)\right)-u_{1, a_{n}^{\alpha}}\left(t^{\prime}, x\right)\right|\right) \\
& \leq P_{0}\left(\left|B\left(t-t^{\prime}\right)\right|>2^{-\frac{3}{2} N(5.8)}\right)^{1 / 2} 8 K e E_{x}\left(e^{2\left|B\left(t-t^{\prime}\right)\right|}+e^{2|x|}\right)^{1 / 2} \\
& \leq c_{3}(K) P_{0}\left(|B(1)|>\left(t-t^{\prime}\right)^{-1 / 8}\right)^{1 / 2} \quad(\text { since }|x| \leq K \text { by ([5.68) }) \\
& \leq c_{4}(K)\left(t-t^{\prime}\right) \\
& \leq c_{5}(K) \sqrt{t-t^{\prime}} \tilde{\Delta}_{u_{1}}\left(m, n, \alpha, \varepsilon_{0}, \eta_{1}, a_{n}^{\frac{\alpha}{2}} \vee 2^{-N}\right),
\end{aligned}
$$

where in the last line we use

$$
\tilde{\Delta}_{u_{1}}\left(m, n, \alpha, \varepsilon_{0}, \eta_{1}, a_{n}^{\frac{\alpha}{2}} \vee 2^{-N}\right) \geq\left(a_{n}^{\frac{\alpha}{2}} \vee 2^{-N}\right)^{1-\frac{\eta_{1}}{2}} \geq 2^{-N} \geq \sqrt{t-t^{\prime}}
$$

(5.83) and (5.84) imply

$$
T_{2} \leq c_{6}(K) \sqrt{t-t^{\prime}}\left[a_{n}^{\beta}+\tilde{\Delta}_{u_{1}}\left(m, n, \alpha, \varepsilon_{0}, \eta_{1}, a_{n}^{\frac{\alpha}{2}} \vee 2^{-N}\right)\right] .
$$

Use (5.77), (5.78) and (5.85) in (5.70) to conclude

$$
\begin{aligned}
& \left|u_{1, a_{n}^{\alpha}}\left(t^{\prime}, x^{\prime}\right)-u_{1, a_{n}^{\alpha}}(t, x)\right| \\
& \leq c_{7}(K) d\left((t, x),\left(t^{\prime}, x^{\prime}\right)\right)\left[a_{n}^{\beta}+\tilde{\Delta}_{u_{1}}\left(m, n, \alpha, \varepsilon_{0}, \eta_{1}, a_{n}^{\frac{\alpha}{2}} \vee 2^{-N}\right)\right] \\
& \quad+2^{-92}\left(t^{\prime}-t\right)^{\frac{1}{2}\left(1-\eta_{1}\right)} a_{n}^{-\varepsilon_{0}} a_{n}^{\alpha / 2} a_{n}^{-3 \alpha / 4}\left[\left(a_{n}^{\alpha / 2} \vee 2^{-N}\right)^{\gamma \gamma \tilde{\gamma}_{m}}+a_{n}^{\beta \gamma}\left(a_{n}^{\alpha / 2} \vee 2^{-N}\right)^{\gamma}\right] .
\end{aligned}
$$

since $\left.d\left((t, x), t^{\prime}, x^{\prime}\right)\right) \leq 2^{-N}$, a bit of arithmetic shows the above is at most

$$
\begin{aligned}
& \left(c_{7}(K) 2^{-N \frac{\eta_{1}}{2}}+2^{-92}\right) d\left((t, x),\left(t^{\prime}, x^{\prime}\right)\right)^{1-\eta_{1}} a_{n}^{-\varepsilon_{0}-3 \alpha / 4} \\
& \times\left\{( a _ { n } ^ { \alpha / 2 } \vee 2 ^ { - N } ) \left[\left(a_{n}^{\alpha / 2} \vee 2^{-N}\right)^{\gamma \tilde{\gamma} m}+a_{n}^{\beta \gamma}\left(a_{n}^{\alpha / 2} \vee 2^{-N}\right)^{\gamma}\right.\right. \\
& \left.\left.+a_{n}^{3 \alpha / 4}\left(a_{n}^{\alpha / 2} \vee 2^{-N}\right)^{\left(\gamma_{m+1}-2\right) \wedge 0}\right]+a_{n}^{3 \alpha / 4} a_{n}^{\beta}\right\} .
\end{aligned}
$$

Choose $N_{1}\left(K, \eta_{1}\right)$ so that

$$
2^{-N_{1} \eta_{1} / 2} c_{7}(K) \leq 2^{-92}
$$

and define $N_{5.13}=N \frac{1 \prime}{5.13} \vee N_{1}$, which is clearly stochastically bounded uniformly in $(n, \alpha, \beta) \in$ $\mathbb{N} \times[0,1] \times\left[0, \frac{1}{2}\right]$. Assume $N \geq N[5.13$. Note that if $m<\bar{m}$, then

$$
\begin{aligned}
a_{n}^{3 \alpha / 4}\left(a_{n}^{\alpha / 2} \vee 2^{-N}\right)^{\left(\gamma_{m+1}-2\right) \wedge 0} & =a_{n}^{3 \alpha / 4}\left(a_{n}^{\alpha / 2} \vee 2^{-N}\right)^{\gamma \gamma_{m}-\frac{3}{2}} \\
& \leq\left(a_{n}^{\alpha / 2} \vee 2^{-N}\right)^{\gamma \gamma_{m}} \\
& \leq\left(a_{n}^{\alpha / 2} \vee 2^{-N}\right)^{\gamma \tilde{\gamma}_{m}},
\end{aligned}
$$


and so the last term in square brackets in (5.86) is bounded by the first term in the same brackets. If $m \geq \bar{m}$, the left-hand side of (5.87) is $a_{n}^{3 \alpha / 4}$. Therefore if $N \geq N \overline{5.13}$ we conclude that

$$
\begin{aligned}
& \left|u_{1, a_{n}^{\alpha}}\left(t^{\prime}, x^{\prime}\right)-u_{1, a_{n}^{\alpha}}(t, x)\right| \\
& \leq 2^{-90} d\left((t, x),\left(t^{\prime}, x^{\prime}\right)\right)^{1-\eta_{1}} a_{n}^{-\varepsilon_{0}-3 \alpha / 4} \\
& \quad \times\left[a_{n}^{\beta+3 \alpha / 4}+a_{n}^{\beta \gamma}\left(a_{n}^{\alpha / 2} \vee 2^{-N}\right)^{\gamma+1}+\left(a_{n}^{\alpha / 2} \vee 2^{-N}\right)^{\gamma \tilde{\gamma}_{m}+1}+1(m \geq \bar{m})\left(a_{n}^{\alpha / 2} \vee 2^{-N}\right) a_{n}^{3 \alpha / 4}\right] \\
& =2^{-90} d\left((t, x),\left(t^{\prime}, x^{\prime}\right)\right)^{1-\eta_{1}} \bar{\Delta}_{u_{1}}\left(m, n, \alpha, \varepsilon_{0}, 2^{-N}\right) .
\end{aligned}
$$

We also require an analogue of the bound on increments on $u_{1, a_{n}^{\alpha}}$ (Proposition [5.13) for $u_{2, a_{n}^{\alpha}}$.

\section{Notation.}

$$
\begin{aligned}
\bar{\Delta}_{1, u_{2}}\left(m, n, \varepsilon_{0}, 2^{-N}\right) & =a_{n}^{-\varepsilon_{0}} 2^{-N \gamma}\left[\left(a_{n}^{1 / 2} \vee 2^{-N}\right)^{\gamma\left(\tilde{\gamma}_{m}-1\right)}+a_{n}^{\beta \gamma}\right] \\
\bar{\Delta}_{2, u_{2}}\left(m, n, \alpha, \varepsilon_{0}\right) & =a_{n}^{-\varepsilon_{0}}\left[a_{n}^{\frac{\alpha}{2}\left(\gamma \tilde{\gamma}_{m}-\frac{1}{2}\right)}+a_{n}^{\beta \gamma} a_{n}^{\frac{\alpha}{2}\left(\gamma-\frac{1}{2}\right)}\right] .
\end{aligned}
$$

We often will suppress the dependence on $\varepsilon_{0}$ and $\alpha$ below.

Proposition 5.14 Let $0 \leq m \leq \bar{m}+1$ and assume $\left(P_{m}\right)$. For any $n \in \mathbb{N}, \eta_{1} \in(0,1 / 2), \varepsilon_{0} \in(0,1)$, $K \in \mathbb{N}^{\geq K_{1}}, \alpha \in[0,1]$, and $\beta \in[0,1 / 2]$, there is an $N_{5.14}=N \sqrt{5.14}\left(m, n, \eta_{1}, \varepsilon_{0}, K, \alpha, \beta\right)(\omega) \in \mathbb{N}$ a.s. such that for all $N \geq N \overline{5.14},(t, x) \in Z(N, n, K, \beta)$, and $t^{\prime} \leq T_{K}$,

$$
\begin{aligned}
& d \equiv d\left((t, x),\left(t^{\prime}, x^{\prime}\right)\right) \leq 2^{-N} \text { implies that } \\
& \left|u_{2, a_{n}^{\alpha}}(t, x)-u_{2, a_{n}^{\alpha}}\left(t^{\prime}, x^{\prime}\right)\right| \leq 2^{-89}\left[d^{\frac{1-\eta_{1}}{2}} \bar{\Delta}_{1, u_{2}}\left(m, n, \varepsilon_{0}, 2^{-N}\right)+d^{1-\eta_{1}} \bar{\Delta}_{2, u_{2}}\left(m, n, \alpha, \varepsilon_{0}\right)\right] .
\end{aligned}
$$

Moreover $N_{5.14}$ is stochastically bounded, uniformly in $(n, \alpha, \beta)$.

The proof is more straightforward than that of Proposition 5.13 and is given in Section 7 below.

Lemma 5.15 For all $n \in \mathbb{N}, 0 \leq \beta \leq 1 / 2$ and $0<d \leq 1$,

$$
a_{n}^{\beta \gamma}\left(\sqrt{a_{n}} \vee d\right)^{\gamma_{1}-1} \leq d \vee a_{n}^{\beta}
$$

Proof. Recall that $\gamma_{1}-1=\gamma-\frac{1}{2}$ and $2 \gamma-\frac{1}{2}>1$.

Case 1. $d \geq a_{n}^{\beta}$.

$a_{n}^{\beta \gamma}\left(\sqrt{a_{n}} \vee d\right)^{\gamma_{1}-1} \leq d^{2 \gamma-\frac{1}{2}} \leq d$.

Case 2. $d<a_{n}^{\beta}$.

$a_{n}^{\beta \gamma}\left(\sqrt{a_{n}} \vee d\right)^{\gamma_{1}-1} \leq a_{n}^{\beta \gamma+\beta\left(\gamma-\frac{1}{2}\right)}=a_{n}^{\beta\left(2 \gamma-\frac{1}{2}\right)} \leq a_{n}^{\beta}$.

We are finally ready to complete the 
Proof of Proposition 5.1. Let $0 \leq m \leq \bar{m}$ and assume $\left(P_{m}\right)$. We must derive $\left(P_{m+1}\right)$. Let $\varepsilon_{0} \in(0,1), M=\left\lceil\frac{2}{\varepsilon_{0}}\right\rceil, \varepsilon_{1}=\frac{1}{M} \leq \varepsilon_{0} / 2$ and set $\alpha_{i}=i \varepsilon_{1}$ for $i=0,1, \ldots, M$, so that $\alpha_{i} \in\left[\varepsilon_{1}, 1\right]$ for $i \geq 1$. Let $n, \xi, K$, and $\beta$ be as in $\left(P_{m}\right)$ where we may assume $\xi>1 / 2$ without loss of generality. Define $\eta_{1}=1-\xi \in(0,1 / 2), \xi^{\prime}=\xi+(1-\xi) / 2 \in(\xi, 1)$,

$$
\begin{gathered}
N_{2}\left(m, n, \xi, \varepsilon_{0}, K, \beta\right)(\omega)=\vee_{i=1}^{M} N_{5.13}\left(m, n, \eta_{1}, \varepsilon_{0} / 2, K+1, \alpha_{i}, \beta\right)(\omega), \\
N_{3}\left(m, n, \xi, \varepsilon_{0}, K, \beta\right)(\omega)=\vee_{i=1}^{M} N_{5.14}\left(m, n, \eta_{1}, \varepsilon_{0} / 2, K+1, \alpha_{i}, \beta\right)(\omega), \\
N_{4}\left(m, n, \xi, \varepsilon_{0}, K, \beta\right)=\left\lceil\frac{2}{1-\xi}\left(\left(N_{5.11}\left(m, n, \eta_{1} / 2, \varepsilon_{0} / 2, K+1, \beta\right) \vee N_{\frac{5.13}{\prime}}^{\prime}\left(\eta_{1} \varepsilon_{1}\right)\right)+1\right)\right\rceil \\
\equiv\left\lceil\frac{1}{1-\xi} N_{5}\left(m, n, \eta_{1}, \varepsilon_{0}, K, \beta\right)\right\rceil,
\end{gathered}
$$

(recall $\varepsilon_{1}$ is a function of $\varepsilon_{0}$ ) and

$$
N_{1}\left(m, n, \xi, \varepsilon_{0}, K, \beta\right)(\omega)=\left(N_{2} \vee N_{3} \vee N_{4}\left(m, n, \xi, \varepsilon_{0}, K, \beta\right)\right) \vee N_{1}\left(0, \xi^{\prime}, K\right)+1 \in \mathbb{N} \text { a.s. }
$$

Recall that in the verification of $\left(P_{0}\right)$, we may take $\varepsilon_{0}=0$ and $N_{1}=N_{1}\left(0, \xi^{\prime}, K\right)$ was independent of $n$ and $\beta$. Then $N_{1}=N_{1}\left(m, n, \xi, \varepsilon_{0}, K, \beta\right)$ is stochastically bounded uniformly in $(n, \beta)$ because $N_{5.11}, N_{5.13}$ and $N_{5.14}$ all are.

Assume

$$
N \geq N_{1},(t, x) \in Z(N, n, K, \beta), t^{\prime} \leq T_{K}, \text { and } d \equiv d\left((t, x),\left(t^{\prime}, x^{\prime}\right)\right) \leq 2^{-N} .
$$

Suppose first that

$$
a_{n}>2^{-N_{5}\left(m, n, \eta_{1}, \varepsilon_{0}, K, \beta\right)} .
$$

Since $N \geq N_{1}\left(0, \xi^{\prime}, K\right)$, we have by $\left(P_{0}\right)$, with $\varepsilon_{0}=0$ and $\xi^{\prime}$ in place of $\xi$, and the fact that $\tilde{\gamma}_{m+1}-1 \leq 1$,

$$
\begin{aligned}
\left|u\left(t^{\prime}, x^{\prime}\right)\right| & \leq 2^{-N \xi^{\prime}} \\
& \leq 2^{-N \xi^{\prime}}\left[\left(\sqrt{a_{n}} \vee 2^{-N}\right)^{\tilde{\gamma}_{m+1}-1}\right] 2^{N_{5} / 2} \\
& \leq 2^{-N(1-\xi) / 2} 2^{N_{5} / 2} 2^{-N \xi}\left[\left(\sqrt{a_{n}} \vee 2^{-N}\right)^{\tilde{\gamma}_{m+1}-1}+a_{n}^{\beta}\right] \\
& \leq 2^{-N \xi}\left[\left(\sqrt{a_{n}} \vee 2^{-N}\right)^{\tilde{\gamma}_{m+1}-1}+a_{n}^{\beta}\right]
\end{aligned}
$$

where in the last line we used $N \geq N_{4} \geq(1-\xi)^{-1} N_{5}$. This proves $\left(P_{m+1}\right)$.

So assume now that

$$
a_{n} \leq 2^{-N_{5}\left(m, n, \eta_{1}, \varepsilon_{0}, K, \beta\right)} .
$$

Let $N^{\prime}=N-1 \geq N_{2} \vee N_{3}$. Note that $\left(\hat{t}_{0}, \hat{x}_{0}\right)$ (the point near $(t, x)$ in the definition of $Z(N, n, K, \beta)$ ) is in $Z(N, n, K+1, \beta) \subset Z\left(N^{\prime}, n, K+1, \beta\right)$ and by the triangle inequality $d\left(\left(\hat{t}_{0}, \hat{x}_{0}\right),\left(t^{\prime}, x^{\prime}\right)\right) \leq 2^{-N^{\prime}}$. (5.90) shows that (5.67) holds with $\left(\varepsilon_{0} / 2, K+1\right)$ in place of $\left(\varepsilon_{0}, K\right)$. Therefore the inequality $N^{\prime} \geq N_{2}$ allows us to apply the conclusion of Proposition 5.13 for $\alpha=\alpha_{i} \geq \varepsilon_{1}, i=1, \ldots, M$ 
with $\left(\hat{t}_{0}, \hat{x}_{0}\right)$ in place of $(t, x), \varepsilon_{0} / 2$ in place of $\varepsilon_{0}$, and $N^{\prime}$ in place of $N$. Simpler reasoning, using $N^{\prime} \geq N_{3}$, allows us to apply the conclusion of Proposition 5.14 with the same parameter values.

Choose $i \in\{1, \ldots, M\}$ so that

$$
\begin{aligned}
& \text { (i) if } 2^{-N^{\prime}}>\sqrt{a_{n}} \text {, then } a_{n}^{\frac{\alpha_{i}}{2}}<2^{-N^{\prime}} \leq a_{n}^{\frac{\alpha_{i-1}}{2}}=a_{n}^{\frac{\alpha_{i}}{2}} a_{n}^{-\frac{\varepsilon_{1}}{2}}, \\
& \text { (ii) if } 2^{-N^{\prime}} \leq \sqrt{a_{n}} \text {, then } i=M \text { and so } a_{n}^{\frac{\alpha_{i}}{2}}=\sqrt{a_{n}} \geq 2^{-N^{\prime}} .
\end{aligned}
$$

In either case we have

$$
a_{n}^{\frac{\alpha_{i}}{2}} \vee 2^{-N^{\prime}} \leq \sqrt{a_{n}} \vee 2^{-N^{\prime}}
$$

and

$$
a_{n}^{-\frac{3 \alpha_{i}}{4}}\left(\sqrt{a_{n}} \vee 2^{-N^{\prime}}\right)^{3 / 2} \leq a_{n}^{-\frac{3 \varepsilon_{1}}{4}} .
$$

Now apply Propositions 5.13 and 5.14, as described above, as well as (5.92), and the facts that $\tilde{\gamma}_{m}=\gamma_{m}$ for $m \leq \bar{m}, \gamma_{m+1}=\gamma \gamma_{m}+\frac{1}{2}$ and $d\left(\left(\hat{t}_{0}, \hat{x}_{0}\right),\left(t^{\prime}, x^{\prime}\right)\right) \leq 2^{-N^{\prime}}$, to conclude

$$
\begin{aligned}
& \left|u\left(\hat{t}_{0}, \hat{x}_{0}\right)-u\left(t^{\prime}, x^{\prime}\right)\right| \\
& \leq\left|u_{1, a_{n}^{\alpha_{i}}}\left(\hat{t}_{0}, \hat{x}_{0}\right)-u_{1, a_{n}^{\alpha_{i}}}\left(t^{\prime}, x^{\prime}\right)\right|+\left|u_{2, a_{n}^{\alpha_{i}}}\left(\hat{t}_{0}, \hat{x}_{0}\right)-u_{2, a_{n}^{\alpha_{i}}}\left(t^{\prime}, x^{\prime}\right)\right| \\
& \leq 2^{-89} a_{n}^{-\frac{\varepsilon_{0}}{2}}\left\{2 ^ { - N ^ { \prime } \xi } \left[a_{n}^{\beta}+a_{n}^{-\frac{3 \alpha_{i}}{4}} a_{n}^{\beta \gamma}\left(\sqrt{a_{n}} \vee 2^{-N^{\prime}}\right)^{(\gamma+1)}+a_{n}^{-\frac{3 \alpha_{i}}{4}}\left(\sqrt{a_{n}} \vee 2^{-N^{\prime}}\right)^{\left(\gamma \gamma_{m}+1\right)}\right.\right. \\
& \left.+1(m=\bar{m})\left(\sqrt{a_{n}} \vee 2^{-N^{\prime}}\right)\right]+2^{-N^{\prime}\left(\frac{\xi}{2}+\gamma\right)}\left[\left(\sqrt{a_{n}} \vee 2^{-N^{\prime}}\right)^{\gamma\left(\gamma_{m}-1\right)}+a_{n}^{\beta \gamma}\right] \\
& \left.+2^{-N^{\prime} \xi}\left[\left(\sqrt{a_{n}} \vee 2^{-N^{\prime}}\right)^{\left(\gamma_{m+1}-1\right)}+a_{n}^{\beta \gamma}\left(\sqrt{a_{n}} \vee 2^{-N^{\prime}}\right)^{\left(\gamma-\frac{1}{2}\right)}\right]\right\} \\
& \leq 2^{-89} a_{n}^{-\frac{\varepsilon_{0}}{2}} 2^{-N^{\prime} \xi}\left\{a_{n}^{\beta}+a_{n}^{-\frac{3 \alpha_{i}}{4}}\left(\sqrt{a_{n}} \vee 2^{-N^{\prime}}\right)^{\frac{3}{2}} a_{n}^{\beta \gamma}\left(\sqrt{a_{n}} \vee 2^{-N^{\prime}}\right)^{\left(\gamma-\frac{1}{2}\right)}\right. \\
& +a_{n}^{-\frac{3 \alpha_{i}}{4}}\left(\sqrt{a_{n}} \vee 2^{-N^{\prime}}\right)^{\frac{3}{2}}\left(\sqrt{a_{n}} \vee 2^{-N^{\prime}}\right)^{\left(\gamma_{m+1}-1\right)}+1(m=\bar{m})\left(\sqrt{a_{n}} \vee 2^{-N^{\prime}}\right) \\
& +\left(\sqrt{a_{n}} \vee 2^{-N^{\prime}}\right)^{\left(\gamma-\frac{1}{2}\right)}\left[\left(\sqrt{a_{n}} \vee 2^{-N^{\prime}}\right)^{\gamma\left(\gamma_{m}-1\right)}+a_{n}^{\beta \gamma}\right] \\
& \left.+\left(\sqrt{a_{n}} \vee 2^{-N^{\prime}}\right)^{\left(\gamma_{m+1}-1\right)}+a_{n}^{\beta \gamma}\left(\sqrt{a_{n}} \vee 2^{-N^{\prime}}\right)^{\left(\gamma-\frac{1}{2}\right)}\right\} .
\end{aligned}
$$

Now apply (5.93) and combine some duplicate terms to bound $\left|u\left(\hat{t}_{0}, \hat{x}_{0}\right)-u\left(t^{\prime}, x^{\prime}\right)\right|$ by $2^{-87} a_{n}^{-\frac{\varepsilon_{0}}{2}-\frac{3 \varepsilon_{1}}{4}} 2^{-N^{\prime} \xi}\left[a_{n}^{\beta}+a_{n}^{\beta \gamma}\left(\sqrt{a_{n}} \vee 2^{-N^{\prime}}\right)^{\gamma-\frac{1}{2}}+\left(\sqrt{a_{n}} \vee 2^{-N^{\prime}}\right)^{\left(\gamma_{m+1}-1\right)}+1(m=\bar{m})\left(\sqrt{a_{n}} \vee 2^{-N^{\prime}}\right)\right]$.

Use the fact that

$$
\left(\sqrt{a_{n}} \vee 2^{-N^{\prime}}\right)^{\left(\gamma_{m+1}-1\right)}+1(m=\bar{m})\left(\sqrt{a_{n}} \vee 2^{-N^{\prime}}\right) \leq 2\left(\sqrt{a_{n}} \vee 2^{-N^{\prime}}\right)\left(\left(\gamma_{m+1} \wedge 2\right)-1\right)
$$

(consider $m<\bar{m}$ and $m=\bar{m}$ separately) and $\varepsilon_{1} \leq \varepsilon_{0} / 2$ in the above to derive

$$
\begin{aligned}
\left|u\left(\hat{t}_{0}, \hat{x}_{0}\right)-u\left(t^{\prime}, x^{\prime}\right)\right| & \leq 2^{-86} a_{n}^{-\varepsilon_{0}} 2^{-N^{\prime} \xi}\left[a_{n}^{\beta}+a_{n}^{\beta \gamma}\left(\sqrt{a_{n}} \vee 2^{-N^{\prime}}\right)^{\left(\gamma-\frac{1}{2}\right)}+\left(\sqrt{a_{n}} \vee 2^{-N^{\prime}}\right)^{\left(\left(\gamma_{m+1} \wedge 2\right)-1\right)}\right] \\
& \leq 2^{-84} a_{n}^{-\varepsilon_{0}} 2^{-N \xi}\left[a_{n}^{\beta}+a_{n}^{\beta \gamma}\left(\sqrt{a_{n}} \vee 2^{-N}\right)^{\left(\gamma-\frac{1}{2}\right)}+\left(\sqrt{a_{n}} \vee 2^{-N}\right)^{\left(\left(\gamma_{m+1} \wedge 2\right)-1\right)}\right] .
\end{aligned}
$$


Finally combine (5.95) and $\left|u\left(\hat{t}_{0}, \hat{x}_{0}\right)\right| \leq \sqrt{a_{n}} 2^{-N}$ to conclude

$$
\begin{aligned}
& \left|u\left(t^{\prime}, x^{\prime}\right)\right| \leq \sqrt{a_{n}} 2^{-N}+2^{-84} a_{n}^{-\varepsilon_{0}} 2^{-N \xi}\left[\sum_{k=0}^{1} a_{n}^{\beta \gamma^{k}}\left(2^{-N} \vee \sqrt{a_{n}}\right)^{\gamma_{k}-1}+\left(2^{-N} \vee \sqrt{a_{n}}\right)^{\tilde{\gamma}_{m+1}-1}\right] \\
& \quad \leq a_{n}^{-\varepsilon_{0}} 2^{-N \xi}\left[\sqrt{a_{n}} 2^{-N(1-\xi)}+2^{-84}\left[\sum_{k=0}^{1} a_{n}^{\beta \gamma^{k}}\left(2^{-N} \vee \sqrt{a_{n}}\right)^{\gamma_{k}-1}+\left(2^{-N} \vee \sqrt{a_{n}}\right)^{\tilde{\gamma}_{m+1}-1}\right]\right] .
\end{aligned}
$$

Our definition of $N_{1}$ (and especially $N_{4}$ ) ensures that $N(1-\xi) \geq 1$ and hence

$$
\sqrt{a_{n}} 2^{-N(1-\xi)} \leq \frac{\sqrt{a_{n}}}{2} \leq \frac{a_{n}^{\beta}}{2}
$$

In addition by Lemma 5.15

$$
a_{n}^{\beta \gamma}\left(\sqrt{a_{n}} \vee 2^{-N}\right)^{\gamma_{1}-1} \leq a_{n}^{\beta} \vee 2^{-N} \leq a_{n}^{\beta}+\left(2^{-N} \vee \sqrt{a_{n}}\right)^{\tilde{\gamma}_{m+1}-1} .
$$

Substitute the last bounds into (5.96) to obtain $\left(P_{m+1}\right)$ and hence complete the induction.

\section{Proof of Proposition 3.3}

We continue to assume $b \equiv 0$ in this Section. Having established the bound $\left(P_{\bar{m}+1}\right)$ in Proposition 5.1, we are now free to use the conclusions of Corollary 5.9, Proposition 5.11 and Proposition 5.14, with $m=\bar{m}+1$, to derive local moduli of continuity for $u_{1, a_{n}^{\alpha}}^{\prime}$ and $u_{2, a_{n}^{\alpha}}$. In view of our main goal, Proposition 3.3, it is the space modulus we will need-the time modulus was only needed to carry out the induction leading to $\left(P_{\bar{m}+1}\right)$.

We fix a $K_{0} \in \mathbb{N}^{\geq K_{1}}$ and positive constants $\varepsilon_{0}, \varepsilon_{1}$ as in (3.10). For $M, n \in \mathbb{N}$ and $0<\beta \leq \frac{1}{2}-\varepsilon_{1}$, define

$$
\alpha=\alpha(\beta)=2\left(\beta+\varepsilon_{1}\right) \in[0,1]
$$

and

$$
\begin{aligned}
U_{M, n, \beta}^{(1)}=\inf \{t & : \text { there are } \varepsilon \in\left[0,2^{-M}\right],|x| \leq K_{0}+1, \hat{x}_{0}, x^{\prime} \in \mathbb{R}, \text { s.t. }\left|x-x^{\prime}\right| \leq 2^{-M}, \\
\left|x-\hat{x}_{0}\right| \leq \varepsilon,\left|u\left(t, \hat{x}_{0}\right)\right| & \leq a_{n} \wedge\left(\sqrt{a_{n}} \varepsilon\right),\left|u_{1, a_{n}}^{\prime}\left(t, \hat{x}_{0}\right)\right| \leq a_{n}^{\beta}, \text { and } \\
\left|u_{1, a_{n}^{\alpha}}^{\prime}(t, x)-u_{1, a_{n}^{\alpha}}^{\prime}\left(t, x^{\prime}\right)\right| & >2^{-82} a_{n}^{-\varepsilon_{0}-\frac{3 \varepsilon_{1}}{2}}\left|x-x^{\prime}\right|^{1-\varepsilon_{0}}\left[a_{n}^{-3 \beta / 2}\left(\varepsilon \vee\left|x^{\prime}-x\right|\right)^{2 \gamma}+1\right. \\
& \left.\left.+a_{n}^{\beta\left(\gamma-\frac{3}{2}\right)}\left(\varepsilon \vee\left|x^{\prime}-x\right|\right)^{\gamma}\right]\right\} \wedge T_{K_{0}} .
\end{aligned}
$$

Define $U_{M, n, 0}^{(1)}$ by the same expression (with $\beta=0$ ) but with the condition on $\left|u_{1, a_{n}^{\alpha}}^{\prime}\left(t, \hat{x}_{0}\right)\right|$ omitted.

$\left\{U_{M, n, \beta}^{(1)}<t\right\}$ is the projection onto $\Omega$ of a Borel $\times \mathcal{F}_{t}$-measurable set in $K \times[0, t] \times \Omega$ where $K$ is a compact subset of $\mathbb{R}^{3}$, and so $U_{M, n, \beta}^{(1)}$ is an $\left(\mathcal{F}_{t}\right)$-stopping time as in IV.T52 of (M66).

Lemma 6.1 For each $n \in \mathbb{N}$ and $\beta$ as in (3.12), $U_{M, n, \beta}^{(1)} \uparrow T_{K_{0}}$ as $M \uparrow \infty$, and in fact

$$
\lim _{M \rightarrow \infty} \sup _{n, 0 \leq \beta \leq \frac{1}{2}-\varepsilon_{1}} P\left(U_{M, n, \beta}^{(1)}<T_{K_{0}}\right)=0 .
$$


Proof. By monotonicity in $M$ the first assertion is immediate from the second. Proposition 5.1 allows us to apply Corollary [5.9] with $m=\bar{m}+1, \eta_{1}=\varepsilon_{0}, K=K_{0}+1$, and $\alpha, \beta$ as in (6.1) and (3.12), respectively. Hence there is an $N_{0}=N_{0}\left(n, \varepsilon_{0}, \varepsilon_{1}, K_{0}+1, \beta\right) \in \mathbb{N}$ a.s., stochastically bounded uniformly in $(n, \beta)$ (as in (3.12) ), and such that if

$$
N \geq N_{0}(\omega),(t, x) \in Z\left(N, n, K_{0}+1, \beta\right),\left|x-x^{\prime}\right| \leq 2^{-N}
$$

then

$$
\begin{aligned}
& \left|u_{1, a_{n}^{\alpha}}^{\prime}(t, x)-u_{1, a_{n}^{\alpha}}^{\prime}\left(t, x^{\prime}\right)\right| \\
& \leq 2^{-85}\left|x-x^{\prime}\right|^{1-\varepsilon_{0}} a_{n}^{-\varepsilon_{0}-\frac{3 \varepsilon_{1}}{2}}\left[a_{n}^{-\frac{3 \beta}{2}} 2^{-2 N \gamma}+1+a_{n}^{\beta\left(\gamma-\frac{3}{2}\right)}\left(a_{n}^{\beta+\varepsilon_{1}} \vee 2^{-N}\right)^{\gamma}\right] .
\end{aligned}
$$

Note that $a_{n}^{-\frac{3 \beta}{2}+\beta \gamma+\left(\beta+\varepsilon_{1}\right) \gamma} \leq 1$ since $\gamma>3 / 4$, and so by the above we have

$$
\begin{aligned}
& \left|u_{1, a_{n}^{\alpha}}^{\prime}(t, x)-u_{1, a_{n}^{\alpha}}^{\prime}\left(t, x^{\prime}\right)\right| \\
& \leq 2^{-84} a_{n}^{-\varepsilon_{0}-\frac{3 \varepsilon_{1}}{2}}\left|x-x^{\prime}\right|^{1-\varepsilon_{0}}\left[a_{n}^{-\frac{3 \beta}{2}} 2^{-2 N \gamma}+1+a_{n}^{\beta\left(\gamma-\frac{3}{2}\right)} 2^{-N \gamma}\right] .
\end{aligned}
$$

Let us assume $\beta>0$ for if $\beta=0$ we can just omit the bound on $\left|u_{1, a_{n}^{\alpha}}^{\prime}\left(t, \hat{x}_{0}\right)\right|$ in what follows. Assume $M \geq N_{0}\left(n, \varepsilon_{0}, \varepsilon_{1}, K_{0}+1, \beta\right)$. Suppose for some $t<T_{K_{0}}\left(\leq T_{K_{0}+1}\right)$ there are $\varepsilon \in\left[0,2^{-M}\right],|x| \leq K_{0}+1, \hat{x}_{0}, x^{\prime} \in \mathbb{R}$ satisfying $\left|x-x^{\prime}\right| \leq 2^{-M},\left|\hat{x}_{0}-x\right| \leq \varepsilon,\left|u\left(t, \hat{x}_{0}\right)\right| \leq a_{n} \wedge\left(\sqrt{a_{n}} \varepsilon\right)$, and $\left|u_{1, a_{n}}^{\prime}\left(t, \hat{x}_{0}\right)\right| \leq a_{n}^{\beta}$. If $x \neq x^{\prime}$, then $0<\left|x-x^{\prime}\right| \vee \varepsilon \leq 2^{-M} \leq 2^{-N_{0}}$ and we may choose $N \geq N_{0}$ so that $2^{-N-1}<\varepsilon \vee\left|x-x^{\prime}\right| \leq 2^{-N}$. Then (6.2) holds and so by (6.3),

$$
\begin{aligned}
\left|u_{1, a_{n}^{\alpha}}^{\prime}(t, x)-u_{1, a_{n}^{\alpha}}^{\prime}\left(t, x^{\prime}\right)\right| \leq 2^{-82} a_{n}^{-\varepsilon_{0}-\frac{3 \varepsilon_{1}}{2}}\left|x-x^{\prime}\right|^{1-\varepsilon_{0}}[ & a_{n}^{-\frac{3 \beta}{2}}\left(\varepsilon \vee\left|x-x^{\prime}\right|\right)^{2 \gamma}+1 \\
& \left.+a_{n}^{\beta\left(\gamma-\frac{3}{2}\right)}\left(\varepsilon \vee\left|x-x^{\prime}\right|\right)^{\gamma}\right] .
\end{aligned}
$$

If $x=x^{\prime}$ the above is trivial. This implies $U_{M, n, \beta}^{(1)}=T_{K_{0}}$ by its definition. We have therefore shown

$$
P\left(U_{M, n, \beta}^{(1)}<T_{K_{0}}\right) \leq P\left(M<N_{0}\right)
$$

This completes the proof because $N_{0}\left(n, \varepsilon_{0}, \varepsilon_{1}, K_{0}+1, \beta\right)$ is stochastically bounded uniformly in $(n, \beta)$ (as in (3.12) ).

Turning next to $u_{2, a_{n}^{\alpha}}$, for $0<\beta \leq \frac{1}{2}-\varepsilon_{1}$, define

$$
\begin{aligned}
U_{M, n, \beta}^{(2)}=\inf \{t & t \text { there are } \varepsilon \in\left[0,2^{-M}\right],|x| \leq K_{0}+1, \hat{x}_{0}, x^{\prime} \in \mathbb{R}, \text { s.t. }\left|x-x^{\prime}\right| \leq 2^{-M}, \\
\left|x-\hat{x}_{0}\right| \leq \varepsilon,\left|u\left(t, \hat{x}_{0}\right)\right| \leq & a_{n} \wedge\left(\sqrt{a_{n}} \varepsilon\right),\left|u_{1, a_{n}}^{\prime}\left(t, \hat{x}_{0}\right)\right| \leq a_{n}^{\beta}, \text { and } \\
\left|u_{2, a_{n}^{\alpha}}(t, x)-u_{2, a_{n}^{\alpha}}\left(t, x^{\prime}\right)\right|> & 2^{-87} a_{n}^{-\varepsilon_{0}}\left(| x - x ^ { \prime } | ^ { \frac { 1 - \varepsilon _ { 0 } } { 2 } } \left[\left(\sqrt{a_{n}} \vee \varepsilon \vee\left|x^{\prime}-x\right|\right)^{2 \gamma}\right.\right. \\
& \left.+a_{n}^{\beta \gamma}\left(\sqrt{a_{n}} \vee \varepsilon \vee\left|x^{\prime}-x\right|\right)^{\gamma}\right] \\
& \left.\left.+\left|x-x^{\prime}\right|^{1-\varepsilon_{0}} a_{n}^{\beta+\frac{\varepsilon_{1}}{4}}\right)\right\} \wedge T_{K_{0} .}
\end{aligned}
$$

Define $U_{M, n, 0}^{(2)}$ by the same expression (with $\beta=0$ ) but with the condition on $\left|u_{1, a_{n}^{\alpha}}^{\prime}\left(t, \hat{x}_{0}\right)\right|$ omitted. Just as for $U^{(1)}, U_{M, n, \beta}^{(2)}$ is an $\mathcal{F}_{t}$-stopping time. 
Lemma 6.2 For each $n \in \mathbb{N}$ and $\beta$ as in (3.12), $U_{M, n, \beta}^{(2)} \uparrow T_{K_{0}}$ as $M \uparrow \infty$, and in fact

$$
\lim _{M \rightarrow \infty} \sup _{n, 0 \leq \beta \leq \frac{1}{2}-\varepsilon_{1}} P\left(U_{M, n, \beta}^{(2)}<T_{K_{0}}\right)=0 .
$$

Proof. As before we only need to show the second assertion. Proposition 5.1 allows us to apply Proposition 5.14 with $m=\bar{m}+1, \eta_{1}=\varepsilon_{0}, K=K_{0}+1$, and $\alpha, \beta$ as in (3.12), (6.1). Hence there is an $N_{0}=N_{0}\left(n, \varepsilon_{0}, \varepsilon_{1}, K_{0}+1, \beta\right) \in \mathbb{N}$ a.s., stochastically bounded uniformly in $(n, \beta)$ (as in (3.12) ), and such that if

$$
N \geq N_{0}(\omega),(t, x) \in Z\left(N, n, K_{0}+1, \beta\right),\left|x-x^{\prime}\right| \leq 2^{-N},
$$

then

$$
\begin{aligned}
& \left|u_{2, a_{n}^{\alpha}}(t, x)-u_{2, a_{n}^{\alpha}}\left(t, x^{\prime}\right)\right| \\
& \leq 2^{-89} a_{n}^{-\varepsilon_{0}}\left\{\left|x-x^{\prime}\right|^{\frac{1-\varepsilon_{0}}{2}}\left[\left(\sqrt{a_{n}} \vee 2^{-N}\right)^{2 \gamma}+a_{n}^{\beta \gamma}\left(\sqrt{a_{n}} \vee 2^{-N}\right)^{\gamma}\right]\right. \\
& \left.\quad+\left|x-x^{\prime}\right|^{1-\varepsilon_{0}}\left[a_{n}^{\left(\beta+\varepsilon_{1}\right)\left(2 \gamma-\frac{1}{2}\right)}+a_{n}^{\beta \gamma} a_{n}^{\left(\beta+\varepsilon_{1}\right)\left(\gamma-\frac{1}{2}\right)}\right]\right\} .
\end{aligned}
$$

Since $\gamma>\frac{3}{4}$,

$$
a_{n}^{\left(\beta+\varepsilon_{1}\right)\left(2 \gamma-\frac{1}{2}\right)}+a_{n}^{\beta \gamma} a_{n}^{\left(\beta+\varepsilon_{1}\right)\left(\gamma-\frac{1}{2}\right)} \leq a_{n}^{\beta+\varepsilon_{1}}+a_{n}^{\beta+\frac{\varepsilon_{1}}{4}} \leq 2 a_{n}^{\beta+\frac{\varepsilon_{1}}{4}} .
$$

Therefore (6.5) shows that (6.4) implies

$$
\begin{aligned}
& \left|u_{2, a_{n}^{\alpha}}(t, x)-u_{2, a_{n}^{\alpha}}\left(t, x^{\prime}\right)\right| \\
& \leq 2^{-89} a_{n}^{-\varepsilon_{0}}\left\{\left|x-x^{\prime}\right|^{\frac{1-\varepsilon_{0}}{2}}\left[\left(\sqrt{a_{n}} \vee 2^{-N}\right)^{2 \gamma}+a_{n}^{\beta \gamma}\left(\sqrt{a_{n}} \vee 2^{-N}\right)^{\gamma}\right]\right. \\
& \left.\quad+2\left|x-x^{\prime}\right|^{1-\varepsilon_{0}} a_{n}^{\beta+\frac{\varepsilon_{1}}{4}}\right\} .
\end{aligned}
$$

The proof is now completed just as for Lemma 6.1 where (6.6) is used in place of (6.3).

\section{Notation.}

$$
\tilde{\Delta}_{u_{1}^{\prime}}\left(n, \varepsilon, \varepsilon_{0}, \beta\right)=a_{n}^{-\varepsilon_{0}} \varepsilon^{-\varepsilon_{0}}\left\{\varepsilon+\left(\varepsilon a_{n}^{-3 / 4}+a_{n}^{-1 / 4}\right)\left(\varepsilon^{2 \gamma}+a_{n}^{\beta \gamma}\left(\varepsilon \vee \sqrt{a_{n}}\right)^{\gamma}\right)\right\} .
$$

For $0<\beta \leq \frac{1}{2}-\varepsilon_{1}$, define

$$
\begin{array}{r}
U_{M, n, \beta}^{(3)}=\inf \left\{t : \text { there are } \varepsilon \in \left[2^{\left.-a_{n}^{-\left(\beta+\varepsilon_{1}\right) \varepsilon_{0} / 4}, 2^{-M}\right],|x| \leq K_{0}+1, \hat{x}_{0} \in \mathbb{R}, \text { s.t. }}\right.\right. \\
\left|x-\hat{x}_{0}\right| \leq \varepsilon,\left|u\left(t, \hat{x}_{0}\right)\right| \leq a_{n} \wedge\left(\sqrt{a_{n}} \varepsilon\right),\left|u_{1, a_{n}}^{\prime}\left(t, \hat{x}_{0}\right)\right| \leq a_{n}^{\beta}, \text { and } \\
\left.\left|u_{1, a_{n}}^{\prime}(t, x)-u_{1, a_{n}^{\alpha}}^{\prime}(t, x)\right|>2^{-78}\left(\tilde{\Delta}_{u_{1}^{\prime}}\left(n, \varepsilon, \varepsilon_{0}, \beta\right)+a_{n}^{\beta+\frac{\varepsilon_{1}}{8}}\right)\right\} \wedge T_{K_{0} .} .
\end{array}
$$

Define $U_{M, n, 0}^{(3)}$ by the same expression (with $\beta=0$ ) but with the condition on $\left|u_{1, a_{n}^{\alpha}}^{\prime}\left(t, \hat{x}_{0}\right)\right|$ omitted.

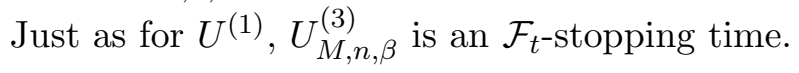

Lemma 6.3 For each $n \in \mathbb{N}$ and $\beta$ as in (3.12), $U_{M, n, \beta}^{(3)} \uparrow T_{K_{0}}$ as $M \uparrow \infty$, and in fact

$$
\lim _{M \rightarrow \infty} \sup _{n, 0 \leq \beta \leq \frac{1}{2}-\varepsilon_{1}} P\left(U_{M, n, \beta}^{(3)}<T_{K_{0}}\right)=0 .
$$


Proof. It suffices to prove the second assertion. By Proposition 5.1 we may apply Proposition 5.11 with $m=\bar{m}+1, \eta_{1}=\varepsilon_{0}, K=K_{0}+1$ and $\beta$ as in (3.12). Note also that if $s=t-a_{n}^{\alpha}+a_{n}$, then

$$
\sqrt{t-s} \leq a_{n}^{\alpha / 2}=a_{n}^{\beta+\varepsilon_{1}}
$$

and

$$
a_{n}^{\beta+\varepsilon_{1}} \leq N^{-4 / \varepsilon_{0}} \Longleftrightarrow 2^{-N} \geq 2^{-a_{n}^{-\left(\beta+\varepsilon_{1}\right) \varepsilon_{0} / 4}}
$$

So Proposition 5.11 shows there is an $N_{0}=N_{0}\left(n, \varepsilon_{0}, K_{0}+1, \beta\right) \in \mathbb{N}$ a.s., stochastically bounded uniformly in $(n, \beta)$, and so that if

$$
N \geq N_{0},(t, x) \in Z\left(N, n, K_{0}+1, \beta\right) \text { and } 2^{-N} \geq 2^{-a_{n}^{-\left(\beta+\varepsilon_{1}\right) \varepsilon_{0} / 4}},
$$

then

$$
\begin{aligned}
& \left|u_{1, a_{n}}^{\prime}(t, x)-u_{1, a_{n}^{\alpha}}^{\prime}(t, x)\right| \\
& =\left|F_{a_{n}}\left(t-a_{n}^{\alpha}+a_{n}, t, x\right)-F_{a_{n}}(t, t, x)\right| \quad(\text { by (5.44) }) \\
& \leq 2^{-81} a_{n}^{-\varepsilon_{0}}\left\{2^{N \varepsilon_{0}}\left[2^{-N}+a_{n}^{-1 / 4}\left(2^{-N} a_{n}^{-1 / 2}+1\right)\left(2^{-2 \gamma N}+a_{n}^{\beta \gamma}\left(2^{-N} \vee \sqrt{a_{n}}\right)^{\gamma}\right)\right]\right. \\
& \left.+a_{n}^{\left(\beta+\varepsilon_{1}\right)\left(1-\varepsilon_{0}\right)}\left[a_{n}^{\left(\beta+\varepsilon_{1}\right)\left(2 \gamma-\frac{3}{2}\right)}+a_{n}^{\beta \gamma} a_{n}^{\left(\beta+\varepsilon_{1}\right)\left(\gamma-\frac{3}{2}\right)}\right]\right\} .
\end{aligned}
$$

We have used $\beta+\varepsilon_{1} \leq \frac{1}{2}$ (from (3.12) $)$ in the last line. The fact that

$$
\left(\beta+\varepsilon_{1}\right)\left(2 \gamma-\frac{3}{2}\right)>\beta \gamma+\left(\beta+\varepsilon_{1}\right)\left(\gamma-\frac{3}{2}\right)
$$

implies that

$$
\begin{aligned}
& a_{n}^{-\varepsilon_{0}} a_{n}^{\left(\beta+\varepsilon_{1}\right)\left(1-\varepsilon_{0}\right)}\left[a_{n}^{\left(\beta+\varepsilon_{1}\right)\left(2 \gamma-\frac{3}{2}\right)}+a_{n}^{\beta \gamma+\left(\beta+\varepsilon_{1}\right)\left(\gamma-\frac{3}{2}\right)}\right] \\
& \leq 2 a_{n}^{-\varepsilon_{0}} a_{n}^{\left(\beta+\varepsilon_{1}\right)\left(1-\varepsilon_{0}\right)+\beta\left(2 \gamma-\frac{3}{2}\right)+\varepsilon_{1}\left(\gamma-\frac{3}{2}\right)} \\
& \leq 2 a_{n}^{\beta\left(2 \gamma-\frac{1}{2}-\varepsilon_{0}\right)+\varepsilon_{1}\left(\gamma-\frac{1}{2}-\varepsilon_{0}\right)-\frac{\varepsilon_{1}}{100}} \\
& \leq 2 a_{n}^{\beta+\frac{\varepsilon_{1}}{8}}
\end{aligned}
$$

where (3.10) is used in the last two inequalities. This allows us to simplify (6.11) and show that (6.10) implies

$$
\left|u_{1, a_{n}}^{\prime}(t, x)-u_{1, a_{n}^{\alpha}}^{\prime}(t, x)\right| \leq 2^{-81}\left[\tilde{\Delta}_{u_{1}^{\prime}, \varepsilon_{0}}\left(n, 2^{-N}, \varepsilon_{0}, \beta\right)+2 a_{n}^{\beta+\frac{\varepsilon_{1}}{8}}\right] .
$$

The proof now is similar to that of Lemma 6.1. As before, we may assume $\beta>0$. Assume $M \geq N_{0}\left(n, \varepsilon_{0}, K_{0}+1, \beta\right)$. Suppose for some $t<T_{K_{0}}$ there are $\varepsilon \in\left[2^{-a_{n}^{-\left(\beta+\varepsilon_{1}\right) \varepsilon_{0} / 4}}, 2^{-M}\right],|x| \leq K_{0}+1$, and $\hat{x}_{0} \in \mathbb{R}$, such that $\left|\hat{x}_{0}-x\right| \leq \varepsilon,\left|u\left(t, \hat{x}_{0}\right)\right| \leq a_{n} \wedge\left(\sqrt{a_{n}} \varepsilon\right)$, and $\left|u_{1, a_{n}}^{\prime}\left(t, \hat{x}_{0}\right)\right| \leq a_{n}^{\beta}$. We may choose $N \geq M \geq N_{0}(\omega)$ so that $2^{-N-1}<\varepsilon \leq 2^{-N}$. Then $(t, x) \in Z\left(N, n, K_{0}+1, \beta\right)$ and 
$2^{-N} \geq \varepsilon \geq 2^{-a_{n}^{-\left(\beta+\varepsilon_{1}\right) \varepsilon_{0} / 4}}$, and therefore (6.10) holds. Therefore we may use (6.12) and the fact that $\tilde{\tilde{\Delta}}\left(n, 2 \varepsilon, \varepsilon_{0}, \beta\right) \leq 8 \tilde{\Delta}\left(n, \varepsilon, \varepsilon_{0}, \beta\right)$ to see that

$$
\left|u_{1, a_{n}}^{\prime}(t, x)-u_{1, a_{n}^{\alpha}}^{\prime}(t, x)\right| \leq 2^{-78}\left[\tilde{\Delta}_{u_{1}^{\prime}}\left(n, \varepsilon, \varepsilon_{0}, \beta\right)+a_{n}^{\beta+\frac{\varepsilon_{1}}{8}}\right] .
$$

This shows that $M \geq N_{0}\left(n, \varepsilon_{0}, K_{0}+1, \beta\right)$ implies $U_{M, n, \beta}^{(3)}=T_{K_{0}}$ and so

$$
\sup _{n, 0 \leq \beta \leq \frac{1}{2}-\varepsilon_{1}} P\left(U_{M, n, \beta}^{(3)}<T_{K_{0}}\right) \leq \sup _{n, 0 \leq \beta \leq \frac{1}{2}-\varepsilon_{1}} P\left(N_{0}>M\right) \rightarrow 0 \text { as } M \rightarrow \infty
$$

by the stochastic boundedness of $N_{0}$ uniformly in $(n, \beta)$.

Finally for $M \in \mathbb{N}$, define

$$
\begin{aligned}
U_{M}^{(4)}=\inf \{t & \text { : there are } \varepsilon \in\left[0,2^{-M}\right],|x| \leq K_{0}+1, \hat{x}_{0}, x^{\prime} \in \mathbb{R}, \text { s.t. }\left|x-x^{\prime}\right| \leq 2^{-M}, \\
& \left.\left|x-\hat{x}_{0}\right| \leq \varepsilon,\left|u\left(t, \hat{x}_{0}\right)\right| \leq \varepsilon, \text { and }\left|u(t, x)-u\left(t, x^{\prime}\right)\right|>\left(\varepsilon \vee\left|x^{\prime}-x\right|\right)^{1-\varepsilon_{0}}\right\} \wedge T_{K_{0}} .
\end{aligned}
$$

Lemma 6.4 $U_{M}^{(4)} \uparrow T_{K_{0}}$ as $M \uparrow \infty$, and

$$
\lim _{M \rightarrow \infty} P\left(U_{M}^{(4)}<T_{K_{0}}\right)=0
$$

Proof. It suffices to prove the second result. This follows easily from Theorem 2.3 as in the proof of Lemma 6.1. The constant multiplicative factors arising in the proof can easily be handled by applying Theorem 2.3 with $\xi=1-\varepsilon_{0} / 2$ in place of $\xi=1-\varepsilon_{0}$.

Let

$$
U_{M, n, \beta}=\wedge_{j=1}^{3} U_{M, n, \beta}^{(j)},
$$

and

$$
U_{M, n}=\left(\wedge_{i=0}^{L\left(\varepsilon_{0}, \varepsilon_{1}\right)} U_{M, n, \beta_{i}}\right) \wedge U_{M}^{(4)},
$$

where we recall that $\left\{\beta_{i}: i \leq L\right\}$ were introduced in (3.11). We have suppressed the dependence of $U_{M, n}$ on our fixed values of $K_{0}, \varepsilon_{0}$ and $\varepsilon_{1}$. Note that $\beta_{i} \in\left[0, \frac{1}{2}-\varepsilon_{1}\right]$ for $i=0, \ldots, L$ by (3.12) and $\alpha_{i}=\alpha\left(\beta_{i}\right)$. Lemmas 6.1, 6.2, 6.3 and 6.4 therefore show that $\left\{U_{M, n}\right\}$ satisfy hypothesis $\left(H_{1}\right)$ of Proposition 2.1. Hence to complete the proof of Proposition 3.3 it suffices to establish compactness of $\tilde{J}_{n, i}(s)$, and the inclusion $\tilde{J}_{n, i}(s) \supset J_{n, i}(s)$ for all $s<U_{M, n},(n, M)$ as in (3.14), and $i=0, \ldots, L$. The next lemmas will show the inclusion part of the proof. We assume $(n, M)$ satisfies (3.14) throughout the rest of this Section.

Lemma 6.5 If $i \in\{0, \ldots, L\}, 0 \leq s<U_{M, n}$, and $x \in J_{n, i}(s)$, then (a) $\mid u_{1, a_{n}}^{\prime}\left(s, \hat{x}_{n}(s, x)\right)-$ $u_{1, a_{n}^{\alpha_{i}}}^{\prime}\left(s, \hat{x}_{n}(s, x)\right) \mid \leq 2^{-74} a_{n}^{\beta_{i}+\frac{\varepsilon_{1}}{8}}$,

(b) for $i>0,\left|u_{1, a_{n}}^{\prime}\left(s, \hat{x}_{n}(s, x)\right)\right| \leq a_{n}^{\beta_{i}} / 2$,

(c) for $i<L, u_{1, a_{n}^{\alpha_{i}}}^{\prime}\left(s, \hat{x}_{n}(s, x)\right) \geq a_{n}^{\beta_{i+1}} / 8$. 
Proof. (a) Assume $(n, i, s, x)$ are as above and set $\varepsilon=\sqrt{a_{n}}$. We have $\left|\left\langle u_{s}, \Phi_{x}^{m_{n+1}}\right\rangle\right| \leq a_{n}$ and

$$
\operatorname{Supp}\left(\Phi_{x}^{m_{n+1}}\right) \subset\left[x-\sqrt{a_{n}}, x+\sqrt{a_{n}}\right] .
$$

Using the continuity of $u(s, \cdot)$, we conclude that

$$
\left|u\left(s, \hat{x}_{n}(s, x)\right)\right| \leq a_{n}=a_{n} \wedge\left(\sqrt{a_{n}} \varepsilon\right), \quad\left|\hat{x}_{n}(s, x)-x\right| \leq \varepsilon .
$$

The definition of $J_{n, i}$ also implies

$$
\left|u_{1, a_{n}}^{\prime}\left(s, \hat{x}_{n}(s, x)\right)\right| \leq a_{n}^{\beta_{i}} / 4 \text { for } i>0 .
$$

In addition, (3.14) and $\varepsilon_{1}<1 / 2($ by (3.10) $)$ imply

$$
2^{-M} \geq \sqrt{a_{n}}=\varepsilon \geq 2^{-a_{n}^{-\varepsilon_{0} \varepsilon_{1} / 4}}
$$

Combine (6.14), (6.15) and (6.16) with $\left|\hat{x}_{n}(s, x)\right| \leq K_{0}+1$ and $s<U_{M, n} \leq U_{M, n, \beta_{i}}^{(3)}$, and take $x=\hat{x}_{0}=\hat{x}_{n}(s, x)$ in the definition of $U^{(3)}$, to conclude that

$$
\left|u_{1, a_{n}}^{\prime}\left(s, \hat{x}_{n}(s, x)\right)-u_{1, a_{n}^{\alpha_{i}}}^{\prime}\left(s, \hat{x}_{n}(s, x)\right)\right| \leq 2^{-78}\left(\tilde{\Delta}_{u_{1}^{\prime}}\left(n, \sqrt{a_{n}}, \varepsilon_{0}, \beta_{i}\right)+a_{n}^{\beta_{i}+\frac{\varepsilon_{1}}{8}}\right) .
$$

Now

$$
\begin{aligned}
\tilde{\Delta}_{u_{1}^{\prime}}\left(n, \sqrt{a_{n}}, \varepsilon_{0}, \beta_{i}\right) & \leq a_{n}^{-3 \varepsilon_{0} / 2}\left[\sqrt{a_{n}}+2 a_{n}^{-1 / 4}\left(a_{n}^{\gamma}+a_{n}^{\gamma\left(\beta_{i}+\frac{1}{2}\right)}\right)\right] \\
& \leq 4 a_{n}^{-3 \varepsilon_{0} / 2}\left[\sqrt{a_{n}}+a_{n}^{\gamma\left(\beta_{i}+\frac{1}{2}\right)-\frac{1}{4}}\right] \quad\left(\text { since } \beta_{i}<\frac{1}{2}\right) \\
& \leq 4\left[a_{n}^{\frac{1-3 \varepsilon_{0}}{2}}+a_{n}^{\beta_{i}-\frac{3 \varepsilon_{0}}{2}+\frac{3 \varepsilon_{1}}{2}}\right] \quad\left(\text { using } \gamma>\frac{3}{4} \text { and } \beta_{i} \leq \frac{1}{2}-6 \varepsilon_{1}\right) \\
& \leq 8 a_{n}^{\beta_{i}+\frac{\varepsilon_{1}}{8}} .
\end{aligned}
$$

The last line follows from (3.10) and a bit of arithmetic. Use the above bound in (6.17) and conclude that

$$
\left|u_{1, a_{n}}^{\prime}\left(s, \hat{x}_{n}(s, x)\right)-u_{1, a_{n}^{\alpha_{i}}}^{\prime}\left(s, \hat{x}_{n}(s, x)\right)\right| \leq 2^{-74} a_{n}^{\beta_{i}+\frac{\varepsilon_{1}}{8}} .
$$

(b) This is immediate from (a) and the fact that $\left|u_{1, a_{n}}^{\prime}\left(s, \hat{x}_{n}(s, x)\right)\right| \leq a_{n}^{\beta_{i}} / 4$ (by the definition of $J_{n, i}$ for $\left.i>0\right)$.

(c) Since $\varepsilon_{0} \leq \varepsilon_{1} / 8$ by (3.10),$a_{n}^{\beta_{i}+\frac{\varepsilon_{1}}{8}} \leq a_{n}^{\beta_{i+1}}$. For $i<L$ we have $u_{1, a_{n}}^{\prime}\left(s, \hat{x}_{n}(s, x)\right) \geq a_{n}^{\beta_{i+1}} / 4$. The result is now clear from (a) and the triangle inequality.

Lemma 6.6 If $i \in\{0, \ldots, L\}, 0 \leq s<U_{M, n}, x \in J_{n, i}(s)$ and $\left|x-x^{\prime}\right| \leq 5 \overline{l_{n}}\left(\beta_{i}\right)$, then

(a) for $i>0,\left|u_{1, a_{n}^{\prime}}^{\prime}\left(s, x^{\prime}\right)\right| \leq a_{n}^{\beta_{i}}$,

(b) for $i<L, u_{1, a_{n}^{\prime}}^{\prime}\left(s, x^{\prime}\right) \geq a_{n}^{\beta_{i+1}} / 16$. 
Proof. Let $\left(n, i, s, x, x^{\prime}\right)$ be as above and set $\varepsilon=\left|x-x^{\prime}\right|+\sqrt{a_{n}}$. Then we have (6.14), (6.15), and also (by (3.14))

$$
\varepsilon \leq 5 a_{n}^{5 \varepsilon_{1}}+\sqrt{a_{n}} \leq 2^{-M}
$$

and

$$
\left|x^{\prime}-\hat{x}_{n}(s, x)\right| \leq \varepsilon \leq 2^{-M}, \quad\left|\hat{x}_{n}(s, x)\right| \leq|x|+1 \leq K_{0}+1 .
$$

(6.14), (6.15), (6.18) and (6.19) allow us to use $s<U_{M, n} \leq U_{M, n, \beta_{i}}^{(1)}$ and the definition of $U_{M, n, \beta_{i}}^{(1)}$ (for $i>0$ or $i=0$ ), with $\hat{x}_{n}(s, x)$ playing the role of $x$, and deduce that

$$
\begin{aligned}
& \left|u_{1, a_{n}^{\alpha_{i}}}^{\prime}\left(s, x^{\prime}\right)-u_{1, a_{n}^{\alpha_{i}}}^{\prime}\left(s, \hat{x}_{n}(s, x)\right)\right| \\
& \begin{aligned}
\leq 2^{-82} a_{n}^{-\varepsilon_{0}-\frac{3 \varepsilon_{1}}{2}}\left(\left|x-x^{\prime}\right|+\sqrt{a_{n}}\right)^{1-\varepsilon_{0}} & {\left[a_{n}^{-3 \beta_{i} / 2}\left(\left|x^{\prime}-x\right|+\sqrt{a_{n}}\right)^{2 \gamma}\right.} \\
& \left.+1+a_{n}^{\beta_{i}\left(\gamma-\frac{3}{2}\right)}\left(\left|x-x^{\prime}\right|+\sqrt{a_{n}}\right)^{\gamma}\right] .
\end{aligned}
\end{aligned}
$$

Use the fact that $\beta_{i}+5 \varepsilon_{1} \leq 1 / 2$ (recall (3.11) ) to infer $\left|x-x^{\prime}\right|+\sqrt{a_{n}} \leq 6 a_{n}^{\beta_{i}+5 \varepsilon_{1}} \leq a_{n}^{\beta_{i}}$ (by (3.14)) and so bound the above by

$$
\begin{aligned}
2^{-79} & a_{n}^{-\varepsilon_{0}-\frac{3 \varepsilon_{1}}{2}} a_{n}^{\left(\beta_{i}+5 \varepsilon_{1}\right)\left(1-\varepsilon_{0}\right)}\left[a_{n}^{\beta_{i}\left(2 \gamma-\frac{3}{2}\right)}+1+a_{n}^{\beta_{i}\left(2 \gamma-\frac{3}{2}\right)}\right] \\
& \leq 2^{-79} a_{n}^{-\varepsilon_{0}-\frac{3 \varepsilon_{1}}{2}} a_{n}^{\left(\beta_{i}+5 \varepsilon_{1}\right)\left(1-\varepsilon_{0}\right)} 3 \\
& \leq 2^{-77} a_{n}^{\beta_{i+1}}
\end{aligned}
$$

provided that $\beta_{i+1} \leq\left(\beta_{i}+5 \varepsilon_{1}\right)\left(1-\varepsilon_{0}\right)-\varepsilon_{0}-\frac{3 \varepsilon_{1}}{2}$, or equivalently

$$
\varepsilon_{0}+\left(\beta_{i}+1\right) \varepsilon_{0} \leq\left((7 / 2)-5 \varepsilon_{0}\right) \varepsilon_{1} .
$$

This follows easily from (3.10). We have therefore shown that

$$
\left|u_{1, a_{n}^{\alpha_{i}}}^{\prime}\left(s, x^{\prime}\right)-u_{1, a_{n}^{\alpha_{i}}}^{\prime}\left(s, \hat{x}_{n}(s, x)\right)\right| \leq 2^{-77} a_{n}^{\beta_{i+1}}
$$

and so both (a) and (b) are now immediate from Lemma 6.5 (b), (c).

Lemma 6.7 If $i \in\{0, \ldots, L\}, 0 \leq s<U_{M, n}, x \in J_{n, i}(s)$, and $\left|x-x^{\prime}\right| \leq 4 \sqrt{a_{n}}$, then

$$
\left|u_{2, a_{n}^{\alpha_{i}}}\left(s, x^{\prime}\right)-u_{2, a_{n}^{\alpha_{i}}}\left(s, x^{\prime \prime}\right)\right| \leq 2^{-75} a_{n}^{\beta_{i+1}}\left(\left|x^{\prime}-x^{\prime \prime}\right| \vee a_{n}^{\gamma-2 \beta_{i}(1-\gamma)-\varepsilon_{1}}\right) \text { whenever }\left|x^{\prime}-x^{\prime \prime}\right| \leq \overline{l_{n}}\left(\beta_{i}\right) .
$$

Proof. Assume $\left(i, n, s, x, x^{\prime}\right)$ are as above and set $\varepsilon=5 \sqrt{a_{n}} \leq 2^{-M}$, by (3.14). Then $\left|x^{\prime}-\hat{x}_{n}(s, x)\right| \leq\left|x^{\prime}-x\right|+\sqrt{a_{n}} \leq \varepsilon, \quad\left|x^{\prime}\right| \leq|x|+1 \leq K_{0}+1, \quad\left|u\left(s, \hat{x}_{n}(s, x)\right)\right| \leq a_{n}=a_{n} \wedge\left(\sqrt{a_{n}} \varepsilon\right)$, and the definition of $(s, x) \in J_{n, i}$ implies that for $i>0$,

$$
\left|u_{1, a_{n}}^{\prime}\left(s, \hat{x}_{n}(s, x)\right)\right| \leq a_{n}^{\beta_{i}} / 4 \leq a_{n}^{\beta_{i}}
$$


Let

$$
Q\left(n, \varepsilon_{0}, \beta_{i}, r\right)=a_{n}^{-\varepsilon_{0}} r^{\frac{1-\varepsilon_{0}}{2}}\left[\left(\sqrt{a_{n}} \vee r\right)^{2 \gamma}+a_{n}^{\beta_{i} \gamma}\left(\sqrt{a_{n}} \vee r\right)^{\gamma}\right] .
$$

Assume $\left|x^{\prime}-x^{\prime \prime}\right| \leq \overline{l_{n}}\left(\beta_{i}\right) \leq 2^{-M}$, the last by (3.14). The condition $s<U_{M, n} \leq U_{M, n, \beta_{i}}^{(2)}$ and the definition of $U^{(2)}$, with $\left(x^{\prime}, x^{\prime \prime}\right)$ playing the role of $\left(x, x^{\prime}\right)$, ensures that

$$
\begin{aligned}
&\left|u_{2, a_{n} \alpha_{i}}\left(s, x^{\prime \prime}\right)-u_{2, a_{n} \alpha_{i}}\left(s, x^{\prime}\right)\right| \leq 2^{-87} a_{n}^{-\varepsilon_{0}}[\left|x^{\prime \prime}-x^{\prime}\right|^{\frac{1-\varepsilon_{0}}{2}}\left[\left(\left(5 \sqrt{a_{n}}\right) \vee\left|x^{\prime \prime}-x^{\prime}\right|\right)^{2 \gamma}\right. \\
&\left.\left.+a_{n}^{\beta_{i} \gamma}\left(\left(5 \sqrt{a_{n}}\right) \vee\left|x^{\prime \prime}-x^{\prime}\right|\right)^{\gamma}\right]+\left|x^{\prime \prime}-x^{\prime}\right|^{1-\varepsilon_{0}} a_{n}^{\beta_{i}+\frac{\varepsilon_{1}}{4}}\right] \\
& \leq 2^{-82}\left[Q\left(n, \varepsilon_{0}, \beta_{i},\left|x^{\prime \prime}-x^{\prime}\right|\right)+\left|x^{\prime \prime}-x^{\prime}\right|^{1-\varepsilon_{0}} a_{n}^{\beta_{i}+\frac{\varepsilon_{1}}{4}-\varepsilon_{0}}\right] .
\end{aligned}
$$

We first show that

$$
Q\left(n, \varepsilon_{0}, \beta_{i}, r\right) \leq 2 a_{n}^{\beta_{i+1}}\left(r \vee a_{n}^{\gamma-2 \beta_{i}(1-\gamma)-\varepsilon_{1}}\right) \text { for } 0 \leq r \leq \overline{l_{n}}\left(\beta_{i}\right) .
$$

Case 1. $\sqrt{a_{n}} \leq r \leq \overline{l_{n}}\left(\beta_{i}\right)$.

$$
Q\left(n, \varepsilon_{0}, \beta_{i}, r\right)=a_{n}^{-\varepsilon_{0}}\left[r^{2 \gamma+\frac{1}{2}-\frac{\varepsilon_{0}}{2}}+a_{n}^{\beta_{i} \gamma} r^{\gamma+\frac{1}{2}-\frac{\varepsilon_{0}}{2}}\right],
$$

and so (6.21), will hold if

$$
r^{2 \gamma-\frac{1}{2}-\frac{\varepsilon_{0}}{2}} \leq a_{n}^{\beta_{i+1}+\varepsilon_{0}},
$$

and

$$
a_{n}^{\beta_{i} \gamma} r^{\gamma-\frac{1}{2}-\frac{\varepsilon_{0}}{2}} \leq a_{n}^{\beta_{i+1}+\varepsilon_{0}} .
$$

(3.10) implies $2 \gamma-\frac{1}{2}-\frac{\varepsilon_{0}}{2}>1$ and so (6.22) would follow from

$$
r \leq a_{n}^{\beta_{i+1}+\varepsilon_{0}} .
$$

Hence, by the upper bound on $r$ in this case, it suffices to show that $a_{n}^{\beta_{i}+5 \varepsilon_{1}} \leq a_{n}^{\beta_{i}+2 \varepsilon_{0}}$ and this is immediate from (3.10).

Turning to (6.23), note that

$$
\begin{aligned}
a_{n}^{\beta_{i} \gamma} r^{\gamma-\frac{1}{2}-\frac{\varepsilon_{0}}{2}} a_{n}^{-\beta_{i+1}-\varepsilon_{0}} & \leq a_{n}^{\beta_{i} \gamma+\left(\beta_{i}+5 \varepsilon_{1}\right)\left(\gamma-\frac{1}{2}-\frac{\varepsilon_{0}}{2}\right)-\beta_{i+1}-\varepsilon_{0}} \\
& \leq a_{n}^{\beta_{i}\left(2 \gamma-\frac{3}{2}-\frac{\varepsilon_{0}}{2}\right)+5 \varepsilon_{1}\left(\gamma-\frac{1}{2}-\frac{\varepsilon_{0}}{2}\right)-2 \varepsilon_{0}} \\
& \leq a_{n}^{5 \varepsilon_{1}\left(\gamma-\frac{1}{2}-\frac{\varepsilon_{0}}{2}\right)-2 \varepsilon_{0}} \leq 1
\end{aligned}
$$

where in the last two inequalities we are using (3.10). This proves (6.23) and hence completes the derivation of (6.21) in this case.

Case 2. $a_{n}^{\gamma-2 \beta_{i}(1-\gamma)-\varepsilon_{1}} \leq r<\sqrt{a_{n}}$.

Then

$$
Q\left(n, \varepsilon_{0}, \beta_{i}, r\right)=a_{n}^{-\varepsilon_{0}} r^{\frac{1-\varepsilon_{0}}{2}}\left[a_{n}^{\gamma}+a_{n}^{\gamma\left(\beta_{i}+\frac{1}{2}\right)}\right] \leq 2 a_{n}^{-\varepsilon_{0}} r^{\frac{1-\varepsilon_{0}}{2}} a_{n}^{\gamma\left(\beta_{i}+\frac{1}{2}\right)}
$$


and so (6.21) will hold if

$$
r^{\frac{1+\varepsilon_{0}}{2}} \geq a_{n}^{-\beta_{i+1}-\varepsilon_{0}+\gamma\left(\beta_{i}+\frac{1}{2}\right)}=a_{n}^{-\beta_{i}(1-\gamma)+\frac{\gamma}{2}-2 \varepsilon_{0}}
$$

Our lower bound on $r$ implies that

$$
r^{\frac{1+\varepsilon_{0}}{2}} \geq a_{n}^{\left(\gamma-2\left(\beta_{i}(1-\gamma)-\varepsilon_{1}\right)\left(1+\varepsilon_{0}\right) / 2\right.} \geq a_{n}^{-\beta_{i}(1-\gamma)+\frac{\gamma}{2}+\frac{\gamma \varepsilon_{0}}{2}-\frac{\varepsilon_{1}}{2}}
$$

which implies (6.24) by (3.10).

Case 3. $r<a_{n}^{\gamma-2 \beta_{i}(1-\gamma)-\varepsilon_{1}}$.

This case follows from Case 2 and the monotonicity of $Q\left(n, \varepsilon_{0}, \beta_{i}, r\right)$ in $r$. Strictly speaking we also need the fact that Case 2 is non-empty as was done in Lemma 3.6.

This completes the proof of (6.21). Consider next the second term in (6.20). If $r \geq a_{n}$, then

$$
\begin{aligned}
r^{1-\varepsilon_{0}} a_{n}^{\beta_{i}+\frac{\varepsilon_{1}}{4}-\varepsilon_{0}}\left(a_{n}^{\beta_{i+1}} r\right)^{-1} & =r^{-\varepsilon_{0}} a_{n}^{-2 \varepsilon_{0}+\frac{\varepsilon_{1}}{4}} \\
& \leq a_{n}^{-3 \varepsilon_{0}+\frac{\varepsilon_{1}}{4}}<1
\end{aligned}
$$

by (3.10). It follows that

$$
r^{1-\varepsilon_{0}} a_{n}^{\beta_{i}+\frac{\varepsilon_{1}}{4}-\varepsilon_{0}} \leq a_{n}^{\beta_{i+1}}\left(r \vee a_{n}\right) \leq a_{n}^{\beta_{i+1}}\left(r \vee a_{n}^{\gamma-2 \beta_{i}(1-\gamma)-\varepsilon_{1}}\right),
$$

the last inequality being trivial.

Insert (6.21) and (6.25) into (6.20) to derive the desired bound.

Lemma 6.8 If $0 \leq s<U_{M, n}$ and $x \in J_{n, 0}(s)$, then

$$
\left|u(s, x)-u\left(s, x^{\prime}\right)\right| \leq\left(\sqrt{a_{n}} \vee\left|x^{\prime}-x\right|\right)^{1-\varepsilon_{0}} \text { whenever }\left|x-x^{\prime}\right| \leq 2^{-M},
$$

and

$$
\left|u\left(s, x^{\prime}\right)\right| \leq 3\left(\sqrt{a_{n}}\right)^{1-\varepsilon_{0}} \text { whenever }\left|x^{\prime}-x\right| \leq \sqrt{a_{n}}
$$

Proof. As in (6.14), if $\varepsilon=\sqrt{a_{n}},(s, x) \in J_{n, 0}$ implies

$$
\left|u\left(s, \hat{x}_{n}(s, x)\right)\right| \leq a_{n} \leq \varepsilon, \quad\left|\hat{x}_{n}(s, x)-x\right| \leq \varepsilon, \text { and }|x| \leq K_{0} .
$$

In addition, (3.14) ensures that $\varepsilon \leq 2^{-M}$, and so $s<U_{M, n} \leq U_{M}^{(4)}$ means that

$$
\left|u\left(s, x^{\prime}\right)-u(s, x)\right| \leq\left(\sqrt{a_{n}} \vee\left|x^{\prime}-x\right|\right)^{1-\varepsilon_{0}} \text { for all }\left|x^{\prime}-x\right| \leq 2^{-M} .
$$

This proves (6.26). Next take $x^{\prime}=\hat{x}_{n}(s, x)$ in the above inequality and use (6.28) to obtain for $\left|x^{\prime}-x\right| \leq \sqrt{a_{n}}$,

$$
\begin{aligned}
\left|u\left(s, x^{\prime}\right)\right| & \leq\left|u\left(s, x^{\prime}\right)-u(s, x)\right|+\left|u(s, x)-u\left(s, \hat{x}_{n}(s, x)\right)\right|+\left|u\left(s, \hat{x}_{n}(s, x)\right)\right| \\
& \leq 2{\sqrt{a_{n}}}^{1-\varepsilon_{0}}+a_{n} \leq 3{\sqrt{a_{n}}}^{1-\varepsilon_{0}} .
\end{aligned}
$$

This proves (6.27). 
Proof of Proposition 3.3 The compactness is elementary and left for the reader-note here that continuity allows us to replace the closed intervals on which the inequalities defining $\tilde{J}_{n, i}(s)$ hold with open intervals.

The inclusions $J_{n, i}(s) \subset \tilde{J}_{n, i}(s)$ for $0 \leq s<U_{M, n}$ are immediate from Lemmas 6.6, 6.7 and 6.8,

This finishes the proof of Proposition 3.3 except for the proof of Proposition 5.14 which is the objective of the next section.

\section{$7 \quad$ Proof of Proposition $\mathbf{5 . 1 4}$}

We now continue to assume $b \equiv 0$ and give the proof of Proposition 5.14. Assume first $t^{\prime} \geq t$ and use (3.5) to write

$$
\begin{aligned}
& \left|u_{2, \delta}\left(t^{\prime}, x^{\prime}\right)-u_{2, \delta}(t, x)\right| \\
& \leq\left|\int_{(t-\delta)^{+}}^{t \wedge\left(t^{\prime}-\delta\right)^{+}} \int p_{t-s}(y-x) D(s, y) W(d s, d y)\right|+\left|\int_{\left(t^{\prime}-\delta\right)^{+} \vee t}^{t^{\prime}} \int p_{t^{\prime}-s}\left(y-x^{\prime}\right) D(s, y) W(d s, d y)\right| \\
& \quad+1\left(t^{\prime}-t<\delta\right)\left|\int_{\left(t^{\prime}-\delta\right)^{+}}^{t} \int\left(p_{t^{\prime}-s}\left(y-x^{\prime}\right)-p_{t-s}(y-x)\right) D(s, y) W(d s, d y)\right| .
\end{aligned}
$$

This decomposition and (3.2) suggests we define the following square functions for $\delta \in(0,1]$ and $\eta_{0} \in(0,1 / 2)$ (noting also that $\left.\left(t^{\prime}-\delta\right)^{+} \vee t \geq t^{\prime}-\left(\delta \wedge\left(t^{\prime}-t\right)\right)\right)$ :

$$
\begin{aligned}
& \hat{Q}_{T, 1, \delta}\left(t, t^{\prime}, x\right)=\int_{(t-\delta)^{+}}^{t \wedge\left(t^{\prime}-\delta\right)^{+}} \int p_{t-s}(y-x)^{2} e^{2 R_{1}|y|}|u(s, y)|^{2 \gamma} d y d s, \\
& \hat{Q}_{T, 2, \delta}\left(t, t^{\prime}, x^{\prime}\right)=\int_{t^{\prime}-\left(\delta \wedge\left(t^{\prime}-t\right)\right)}^{t^{\prime}} \int p_{t^{\prime}-s}\left(y-x^{\prime}\right)^{2} e^{2 R_{1}|y|}|u(s, y)|^{2 \gamma} d y d s, \\
& \hat{Q}_{S, 1, \delta, \eta_{0}}\left(t, x, t^{\prime}, x^{\prime}\right)=1\left(t^{\prime}-t<\delta\right) \int_{\left(t^{\prime}-\delta\right)^{+}}^{t} \int 1\left(|y-x|>\left(t^{\prime}-s\right)^{1 / 2-\eta_{0}} \vee\left(2\left|x-x^{\prime}\right|\right)\right) \\
& \times\left(p_{t^{\prime}-s}\left(y-x^{\prime}\right)-p_{t-s}(y-x)\right)^{2} e^{2 R_{1}|y|}|u(s, y)|^{2 \gamma} d y d s, \\
& \hat{Q}_{S, 2, \delta, \eta_{0}}\left(t, x, t^{\prime}, x^{\prime}\right)=1\left(t^{\prime}-t<\delta\right) \\
& \int_{\left(t^{\prime}-\delta\right)^{+}}^{t} \int 1\left(|y-x| \leq\left(t^{\prime}-s\right)^{1 / 2-\eta_{0}} \vee\left(2\left|x-x^{\prime}\right|\right)\right) \\
& \times\left(p_{t^{\prime}-s}\left(y-x^{\prime}\right)-p_{t-s}(y-x)\right)^{2} e^{2 R_{1}|y|}|u(s, y)|^{2 \gamma} d y d s .
\end{aligned}
$$

Lemma 7.1 For any $K \in \mathbb{N}^{\geq K_{1}}$ and $R>2$ there is a $77.1(K, R)>0$ and an $N$ 7.1 $=N$ 7.1 $(K, \omega) \in$ $\mathbb{N}$ a.s. such that for all $\eta_{0}, \eta_{1} \in(1 / R, 1 / 2), \delta \in(0,1], N, n \in \mathbb{N}, \beta \in[0,1 / 2]$ and $(t, x) \in \mathbb{R}_{+} \times \mathbb{R}$, on

$$
\{\omega:(t, x) \in Z(N, n, K, \beta), N \geq N \text { 7.1] }
$$




$$
\hat{Q}_{S, 1, \delta, \eta_{0}}\left(t, x, t^{\prime}, x^{\prime}\right) \leq q_{7.1}(K, R) 2^{4 \Lambda} \overline{7.1}\left[d\left((t, x),\left(t^{\prime}, x^{\prime}\right)\right) \wedge \sqrt{\delta}\right]^{2-\eta_{1}} \delta^{3 / 2} \quad \text { for all } t \leq t^{\prime} \text { and } x^{\prime} \in \mathbb{R} .
$$

Proof. The proof is quite similar to that of Lemma [5.4. We let $d=d\left((t, x),,\left(t^{\prime}, x^{\prime}\right)\right)$ and $N_{7.1}=$ $N_{1}(0,3 / 4, K)$, where $N_{1}$ is as in $\left(P_{0}\right)$. Recall here from Remark 5.3 that for $m=0, N_{1}$ depends only on $(\xi, K)$ and we take $\xi=3 / 4$. We may assume $t^{\prime}-t<\delta$. Then for $\omega$ as in (7.2) and $t \leq t^{\prime}$, Lemma 5.2, with $m=0$, implies

$$
\begin{aligned}
& \hat{Q}_{S, 1, \delta, \eta_{0}}\left(t, x, t^{\prime}, x^{\prime}\right) \\
& \leq C_{5.2}(\omega) \int_{\left(t^{\prime}-\delta\right)^{+}}^{t} \int 1\left(|y-x|>\left(t^{\prime}-s\right)^{1 / 2-\eta_{0}} \vee\left(2\left|x-x^{\prime}\right|\right)\right)\left(p_{t^{\prime}-s}\left(y-x^{\prime}\right)-p_{t-s}(y-x)\right)^{2} \\
& \times e^{2\left(|y-x|+R_{1}|y|\right)}\left(2^{-N} \vee(\sqrt{t-s}+|y-x|)\right)^{3 \gamma / 2} d y d s \\
& \leq C_{5.2}(\omega) \int_{\left(t^{\prime}-\delta\right)^{+}}^{t} \int 1\left(|y-x|>\left(t^{\prime}-s\right)^{1 / 2-\eta_{0}} \vee\left(2\left|x-x^{\prime}\right|\right)\right)\left(p_{t^{\prime}-s}\left(y-x^{\prime}\right)-p_{t-s}(y-x)\right)^{2} \\
& e^{2 R_{1} K} e^{2\left(R_{1}+1\right)|y-x|}[1+|y-x|]^{3 \gamma / 2} d y d s \\
& \leq c_{1}(K, R) q_{5.2}(\omega) \int_{t^{\prime}-\delta}^{t}(t-s)^{-1 / 2} \exp \left(-\frac{\eta_{1}\left(t^{\prime}-s\right)^{-2 \eta_{0}}}{33}\right)\left[1 \wedge \frac{d^{2}}{t-s}\right]^{1-\frac{\eta_{1}}{2}} d s,
\end{aligned}
$$

where we have used Lemma 4.3 (b) in the last line. Now use

$$
\exp \left(-\frac{\eta_{1}\left(t^{\prime}-s\right)^{-2 \eta_{0}}}{33}\right) \leq \exp \left(-\frac{\eta_{1}\left(t^{\prime}-t\right)^{-2 \eta_{0}}}{66}\right)+\exp \left(-\frac{\eta_{1}(t-s)^{-2 \eta_{0}}}{66}\right)
$$

to bound the above by

$$
\begin{gathered}
c_{1}(K, R) C_{5.2}(\omega)\left[\exp \left(-\frac{\eta_{1}\left(t^{\prime}-t\right)^{-2 \eta_{0}}}{66}\right) \int_{t^{\prime}-\delta}^{t}(t-s)^{-1 / 2}\left[1 \wedge \frac{d^{2}}{t-s}\right]^{1-\frac{\eta_{1}}{2}} d s\right. \\
\left.+\int_{t^{\prime}-\delta}^{t}(t-s)^{-1 / 2} \exp \left(-\frac{\eta_{1}(t-s)^{-2 \eta_{0}}}{66}\right)\left[1 \wedge \frac{d^{2}}{t-s}\right]^{1-\frac{\eta_{1}}{2}} d s\right] \\
\leq c_{2}(K, R) C_{5.2}(\omega)\left[\exp \left(-\frac{\eta_{1}\left(t^{\prime}-t\right)^{-2 \eta_{0}}}{66}\right)\left(d^{2} \wedge \delta\right)^{1 / 2}\right. \\
\left.+\int_{t^{\prime}-\delta}^{t}(t-s)^{3 / 2}\left[1 \wedge \frac{d^{2}}{t-s}\right]^{1-\frac{\eta_{1}}{2}} d s\right] \quad(\text { use (4.2) }) \\
\left.\leq c_{3}(K, R) C_{5.2}(\omega)\left(d^{2} \wedge \delta\right)^{1-\frac{\eta_{1}}{2}} \delta^{3 / 2} \quad \text { (use (4.1) and } t^{\prime}-t \leq d^{2} \wedge \delta\right) .
\end{gathered}
$$

As we may take $\varepsilon_{0}=0$ in the formula for $C_{5.2}(\omega)$ (by Remark 5.3 ), the result follows.

Lemma 7.2 Let $0 \leq m \leq \bar{m}+1$ and assume $\left(P_{m}\right)$. For any $K \in \mathbb{N} \geq K_{1}, R>2, n \in \mathbb{N}, \varepsilon_{0} \in(0,1)$, and $\beta \in[0,1 / 2]$ there is a 7 7.2 $(K)$ and $N$ 7.2 $=N$ 7.2 $\left(m, n, R, \varepsilon_{0}, K, \beta\right)(\omega) \in \mathbb{N}$ a.s. such that for any $\eta_{1} \in\left(R^{-1}, 1 / 2\right), \eta_{0} \in\left(0, \eta_{1} / 24\right), \delta \in\left[a_{n}, 1\right], N \in \mathbb{N}$, and $(t, x) \in \mathbb{R}_{+} \times \mathbb{R}$, on

$$
\{\omega:(t, x) \in Z(N, n, K, \beta), N \geq N \text { 7.2] },
$$




$$
\begin{aligned}
& \hat{Q}_{S, 2, \delta, \eta_{0}}\left(t, x, t^{\prime}, x^{\prime}\right) \\
& \leq 17.2(K)\left[a_{n}^{-2 \varepsilon_{0}}+2^{4 N \overline{7.2}][}\left[(d \wedge \sqrt{\delta})^{1-\frac{\eta_{1}}{2}} \vec{d}_{N}^{2 \gamma}\left[\vec{d}_{n, N}^{2 \gamma\left(\tilde{\gamma}_{m}-1\right)}+a_{n}^{2 \beta \gamma}\right]\right.\right. \\
& \left.+(d \wedge \sqrt{\delta})^{2-\eta_{1}}\left[\delta^{\gamma \tilde{\gamma}_{m}-\frac{1}{2}}+a_{n}^{2 \beta \gamma} \delta^{\gamma-\frac{1}{2}}\right]\right] \\
& \text { for all } t \leq t^{\prime} \leq K,\left|x^{\prime}\right| \leq K+1 .
\end{aligned}
$$

Here $d=d\left((t, x),\left(t^{\prime}, x^{\prime}\right)\right), \bar{d}_{N}=d \vee 2^{-N}$ and $\bar{d}_{n, N}=\sqrt{a_{n}} \vee \bar{d}_{N}$. Moreover $N$ 7.2 is stochastically bounded uniformly in $(n, \beta)$.

Proof. Set $\xi=1-(24 R)^{-1}$ and $N \overline{7.2}\left(m, n, R, \varepsilon_{0}, K, \beta\right)=N_{1}\left(m, n, \xi, \varepsilon_{0}, K, \beta\right)$, which is clearly stochastically bounded uniformly in $(n, \beta)$ by $\left(P_{m}\right)$. We may assume $t^{\prime}-t \leq \delta$. For $\omega$ as in (17.3), $t \leq t^{\prime} \leq K$ and $\left|x^{\prime}\right| \leq K+1$, Lemma 5.2 implies

$$
\begin{aligned}
& \hat{Q}_{S, 2, \delta, \eta_{0}}\left(t, x, t^{\prime}, x^{\prime}\right) \\
& \leq q_{5.2}(\omega) c_{1}(K) \int_{\left(t^{\prime}-\delta\right)^{+}}^{t}\left[\int\left(p_{t^{\prime}-s}\left(y-x^{\prime}\right)-p_{t-s}(y-x)\right)^{2} d y\right] e^{2 R_{1} K} e^{2\left(R_{1}+1\right) 4(K+1)} \\
& \times\left(\left(2^{-N} \vee\left|x^{\prime}-x\right|\right)+\left(t^{\prime}-s\right)^{\frac{1}{2}-\eta_{0}}\right)^{2 \gamma \xi}\left[\left(\left(\sqrt{a_{n}} \vee 2^{-N} \vee\left|x^{\prime}-x\right|\right)\right.\right. \\
& \left.\left.+\left(t^{\prime}-s\right)^{\frac{1}{2}-\eta_{0}}\right)^{2 \gamma\left(\tilde{\gamma}_{m}-1\right)}+a_{n}^{2 \beta \gamma}\right] d s \\
& \leq 9_{5.2}(\omega) c_{2}(K) \int_{\left(t^{\prime}-\delta\right)^{+}}^{t}(t-s)^{-1 / 2}\left[1 \wedge \frac{d^{2}}{t-s}\right]\left(\left(2^{-N} \vee d^{1-2 \eta_{0}}\right)+(t-s)^{\frac{1}{2}-\eta_{0}}\right)^{2 \gamma \xi} \\
& \times\left[\left(\left(\sqrt{a_{n}} \vee 2^{-N} \vee d^{1-2 \eta_{0}}\right)+(t-s)^{\frac{1}{2}-\eta_{0}}\right)^{2 \gamma\left(\tilde{\gamma}_{m}-1\right)}+a_{n}^{2 \beta \gamma}\right] d s .
\end{aligned}
$$

We have used Lemma 4.3(a) in the last line. Below we will implicitly use the conditions on $\eta_{0}, \eta_{1}$, $R$ and $\gamma$ to see that

$$
\left(1-2 \eta_{0}\right) \gamma \xi>\left(\frac{23}{24}\right)\left(\frac{3}{4}\right)\left(\frac{47}{48}\right)>\frac{1}{2}
$$

and also use the conditions on $t, x, t^{\prime}, x^{\prime}$ which imply $d \leq c(K)$ (the latter was also used in (7.4)). By considering separately the cases

$$
\begin{aligned}
& (t-s)^{\frac{1}{2}-\eta_{0}}<2^{-N} \vee d^{1-2 \eta_{0}}, \quad(t-s)^{\frac{1}{2}-\eta_{0}} \geq \sqrt{a_{n}} \vee 2^{-N} \vee d^{1-2 \eta_{0}} \\
& \text { and } 2^{-N} \vee d^{1-2 \eta_{0}} \leq(t-s)^{\frac{1}{2}-\eta_{0}}<\sqrt{a_{n}} \vee 2^{-N} \vee d^{1-2 \eta_{0}}
\end{aligned}
$$


(the latter case implies $\sqrt{a_{n}}>2^{-N} \vee d^{1-2 \eta_{0}}$ ), and then using Lemma 4.1 we may bound (7.4) by

$$
\begin{aligned}
& 95.2(\omega) c_{3}(K)\left\{\int_{\left(t^{\prime}-\delta\right)^{+}}^{t}(t-s)^{-1 / 2}\left[1 \wedge \frac{d^{2}}{t-s}\right] d s\left(2^{-N} \vee d^{1-2 \eta_{0}}\right)^{2 \gamma \xi}\right. \\
& \times\left[\left(\sqrt{a_{n}} \vee 2^{-N} \vee d^{1-2 \eta_{0}}\right)^{2 \gamma\left(\tilde{\gamma}_{m}-1\right)}+a_{n}^{2 \beta \gamma}\right] \\
& +\int_{\left(t^{\prime}-\delta\right)^{+}}^{t}\left[(t-s)^{-\frac{1}{2}+\left(1-2 \eta_{0}\right) \gamma\left(\tilde{\gamma}_{m}-1+\xi\right)}+a_{n}^{2 \beta \gamma}(t-s)^{-\frac{1}{2}+\left(1-2 \eta_{0}\right) \gamma \xi}\right]\left[1 \wedge \frac{d^{2}}{t-s}\right] d s \\
& \left.+\int_{\left(t^{\prime}-\delta\right)^{+}}^{t}(t-s)^{\left(1-2 \eta_{0}\right) \gamma \xi-\frac{1}{2}}\left[1 \wedge \frac{d^{2}}{t-s}\right] d s\left[a_{n}^{\gamma\left(\tilde{\gamma}_{m}-1\right)}+a_{n}^{2 \beta \gamma}\right]\right\} \\
& \leq C_{5.2}(\omega) c_{4}(K)\left\{\left(d^{2} \wedge \delta\right)^{1 / 2}\left(2^{-N} \vee d^{1-2 \eta_{0}}\right)^{2 \gamma \xi}\right. \\
& \quad \times\left[\left(\sqrt{a_{n}} \vee 2^{-N} \vee d^{1-2 \eta_{0}}\right)^{2 \gamma\left(\tilde{\gamma}_{m}-1\right)}+a_{n}^{2 \beta \gamma}\right] \\
& +\left(d^{2} \wedge \delta\right)\left[\delta^{\left(1-2 \eta_{0}\right) \gamma\left(\tilde{\gamma}_{m}-1+\xi\right)-\frac{1}{2}}+a_{n}^{2 \beta \gamma} \delta^{\left(1-2 \eta_{0}\right) \gamma \xi-\frac{1}{2}}\right] \\
& \left.+\left(d^{2} \wedge \delta\right) \delta^{\left(1-2 \eta_{0}\right) \gamma \xi-\frac{1}{2}}\left[a_{n}^{\gamma\left(\tilde{\gamma}_{m}-1\right)}+a_{n}^{2 \beta \gamma}\right]\right\}
\end{aligned}
$$

(by (4.2) and (4.1), respectively).

The last term is less than the middle term because $\delta \in\left[a_{n}, 1\right]$. Therefore $\hat{Q}_{S, 2, \delta, \eta_{0}}\left(t, x, t^{\prime}, x^{\prime}\right)$ is at most

$$
\begin{aligned}
95.2(\omega) c_{5}(K)\{(d & \wedge \sqrt{\delta})^{1-\frac{\eta_{1}}{2}}\left(2^{-N} \vee d\right)^{\left(1-2 \eta_{0}\right) 2 \gamma \xi+\frac{\eta_{1}}{4}} \\
\times & {\left[\left(\sqrt{a_{n}} \vee 2^{-N} \vee d\right)^{\left(1-2 \eta_{0}\right) 2 \gamma\left(\tilde{\gamma}_{m}-1\right)+\frac{\eta_{1}}{4}}+a_{n}^{2 \beta \gamma}\left(\sqrt{a_{n}} \vee 2^{-N} \vee d\right)^{\frac{\eta_{1}}{4}}\right] } \\
& \left.+\left(d^{2} \wedge \delta\right)^{1-\frac{\eta_{1}}{2}}\left[\delta^{\left(1-2 \eta_{0}\right) \gamma\left(\tilde{\gamma}_{m}-1+\xi\right)-\frac{1}{2}+\frac{\eta_{1}}{2}}+a_{n}^{2 \beta \gamma} \delta^{\left(1-2 \eta_{0}\right) \gamma \xi-\frac{1}{2}+\frac{\eta_{1}}{2}}\right]\right\}
\end{aligned}
$$

Our conditions on $\eta_{0}, \eta_{1}$, and $R$ imply

$$
\begin{aligned}
& \left(1-2 \eta_{0}\right) 2 \gamma \xi+\frac{\eta_{1}}{4} \geq 2 \gamma, \quad\left(1-2 \eta_{0}\right) 2 \gamma\left(\tilde{\gamma}_{m}-1\right)+\frac{\eta_{1}}{4} \geq 2 \gamma\left(\tilde{\gamma}_{m}-1\right) \\
& \left(1-2 \eta_{0}\right) \gamma\left(\tilde{\gamma}_{m}-1+\xi\right)+\frac{\eta_{1}}{2} \geq \gamma \tilde{\gamma}_{m}, \text { and }\left(1-2 \eta_{0}\right) \gamma \xi+\frac{\eta_{1}}{2} \geq \gamma
\end{aligned}
$$

Finally, insert the above bounds into (17.5) to derive the required bound on $\hat{Q}_{S, 2, \delta, \eta_{0}}\left(t, x, t^{\prime}, x^{\prime}\right)$.

Lemma 7.3 Let $0 \leq m \leq \bar{m}+1$ and assume $\left(P_{m}\right)$. For any $K \in \mathbb{N} \geq K_{1}, R>2, n \in \mathbb{N}, \varepsilon_{0} \in(0,1)$, and $\beta \in[0,1 / 2]$ there is a $77.3(K)$ and $N$ 7.3 $=N$ 7.3. $\left(m, n, R, \varepsilon_{0}, K, \beta\right)(\omega) \in \mathbb{N}$ a.s. such that for any $\eta_{1} \in\left(R^{-1}, 1 / 2\right), \delta \in\left[a_{n}, 1\right], N \in \mathbb{N}$, and $(t, x) \in \mathbb{R}_{+} \times \mathbb{R}$,

$$
\text { on }\left\{\omega:(t, x) \in Z(N, n, K, \beta), N \geq N \overline{\text { 7.g. }} \text {, and for all } t \leq t^{\prime} \leq T_{K},\left|x^{\prime}\right| \leq K+1\right. \text {, }
$$




$$
\begin{aligned}
& \hat{Q}_{T, 1, \delta}\left(t, t^{\prime}, x\right) \\
& \leq \text { 7.3. }(K)\left[a_{n}^{-2 \varepsilon_{0}}+2^{4 N \overline{7.3}]}\left[\left(\sqrt{t^{\prime}-t} \wedge \sqrt{\delta}\right)^{1-\frac{\eta_{1}}{2}} \vec{d}_{N}^{2 \gamma}\left[\vec{d}_{n, N}^{2 \gamma\left(\tilde{\gamma}_{m}-1\right)}+a_{n}^{2 \beta \gamma}\right]\right.\right. \\
& \left.+\left(\sqrt{t^{\prime}-t} \wedge \sqrt{\delta}\right)^{2-\eta_{1}}\left[\delta^{\gamma \tilde{\gamma}_{m}-\frac{1}{2}}+a_{n}^{2 \beta \gamma} \delta^{\gamma-\frac{1}{2}}\right]\right],
\end{aligned}
$$

and

$$
\begin{aligned}
& \hat{Q}_{T, 2, \delta}\left(t, t^{\prime}, x^{\prime}\right) \\
& \leq q 7.3(K)\left[a_{n}^{-2 \varepsilon_{0}}+2^{4 \Lambda}[\bar{\gamma} .3]\left(\sqrt{t^{\prime}-t} \wedge \sqrt{\delta}\right)^{1-\frac{\eta_{1}}{2}} \vec{d}_{N}^{2 \gamma}\left[\vec{d}_{n, N}^{2 \gamma\left(\tilde{\gamma}_{m}-1\right)}+a_{n}^{2 \beta \gamma}\right] .\right.
\end{aligned}
$$

Here $d, \bar{d}_{N}$ and $\bar{d}_{n, N}$ are as in Lemma 7.2. Moreover, $N$ 7.3 is stochastically bounded uniformly in $(n, \beta)$.

Proof. Set $\xi=1-(4 R)^{-1}$ and $N_{7.3}\left(m, n, R, \varepsilon_{0}, K, \beta\right)=N_{1}\left(m, n, \xi, \varepsilon_{0}, K, \beta\right)$, which is clearly stochastically bounded uniformly in $(n, \beta)$ by $\left(P_{m}\right)$. For $\omega, t, x, t^{\prime}$ and $x^{\prime}$ as in (7.6), Lemma 5.2 gives

$$
\begin{aligned}
& \hat{Q}_{T, 2, \delta}\left(t, t^{\prime}, x^{\prime}\right) \\
& \leq C_{\underline{5.2}}(\omega) c_{1} \int_{t^{\prime}-\left(\delta \wedge\left(t^{\prime}-t\right)\right)}^{t^{\prime}} \int p_{t^{\prime}-s}\left(y-x^{\prime}\right)^{2} e^{2 R_{1} K+\left(2\left(R_{1}+1\right)\right)\left|y-x^{\prime}\right|+2\left(R_{1}+1\right)(2 K+1)} \\
& \times\left[2^{-N} \vee\left|x-x^{\prime}\right|+\sqrt{t^{\prime}-s}+\left|y-x^{\prime}\right|\right]^{2 \gamma \xi} \\
& \times\left\{\left[\sqrt{a_{n}} \vee 2^{-N} \vee\left|x-x^{\prime}\right|+\sqrt{t^{\prime}-s}+\left|y-x^{\prime}\right|\right]^{2 \gamma\left(\tilde{\gamma}_{m}-1\right)}+a_{n}^{2 \beta \gamma}\right\} d y d s \\
& \leq C_{[5.2}(\omega) c_{2}(K) \int_{t^{\prime}-\left(\delta \wedge\left(t^{\prime}-t\right)\right)}^{t^{\prime}}\left(t^{\prime}-s\right)^{-1 / 2}\left[\left(2^{-N} \vee\left|x-x^{\prime}\right|\right)^{2 \gamma \xi}+\left(t^{\prime}-s\right)^{\gamma \xi}\right] \\
& \times\left\{\left(\left(\sqrt{a_{n}} \vee 2^{-N} \vee\left|x-x^{\prime}\right|\right)^{2 \gamma\left(\tilde{\gamma}_{m}-1\right)}+\left(t^{\prime}-s\right)^{\gamma\left(\tilde{\gamma}_{m}-1\right)}\right)+a_{n}^{2 \beta \gamma}\right\} d s .
\end{aligned}
$$

For $t \leq s \leq t^{\prime}$ and $c, p \geq 0$,

$$
\left(c \vee\left|x-x^{\prime}\right|\right)^{p}+\left(t^{\prime}-s\right)^{p / 2} \leq 2(c \vee d)^{p} .
$$

Use this with $c=2^{-N}$ or $\sqrt{a_{n}} \vee 2^{-N}$ to bound (7.7) by

$$
\begin{aligned}
& C_{5.2}(\omega) c_{3}(K)\left(\left(t^{\prime}-t\right) \wedge \delta\right)^{\frac{1}{2}}\left[2^{-N} \vee d\right]^{2 \gamma \xi}\left\{\left(\sqrt{a_{n}} \vee 2^{-N} \vee d\right)^{2 \gamma\left(\tilde{\gamma}_{m}-1\right)}+a_{n}^{2 \beta \gamma}\right\} \\
& \leq C_{5.2}(\omega) c_{3}(K)\left(\sqrt{t^{\prime}-t} \wedge \sqrt{\delta}\right)^{1-\frac{\eta_{1}}{2}} \bar{d}_{N}^{2 \gamma \xi+\frac{\eta_{1}}{2}}\left\{\vec{d}_{n, N}^{2 \gamma\left(\tilde{\gamma}_{m}-1\right)}+a_{n}^{2 \beta \gamma}\right\} .
\end{aligned}
$$

The conditions on $\eta_{1}$ and definition of $\xi$ imply $2 \gamma \xi+\frac{\eta_{1}}{2} \geq 2 \gamma$, and so the bound on $\hat{Q}_{T, 2, \delta}$ is established. 
Turning to $\hat{Q}_{T, 1, \delta}$, we may assume $t^{\prime}>\delta$, or else $\hat{Q}_{T, 1, \delta}=0$. Argue as in the derivation of (7.7) to see that for $\omega, t, t^{\prime}$ and $x$ as in (7.6),

$$
\begin{aligned}
& \hat{Q}_{T, 1, \delta}\left(t, t^{\prime}, x\right) \leq C_{[5.2}(\omega) c_{4}(K) \int_{(t-\delta)^{+}}^{t \wedge\left(t^{\prime}-\delta\right)}(t-s)^{-1 / 2}\left[\left(2^{-2 N \gamma \xi}+(t-s)^{\gamma \xi}\right]\right. \\
& \times\left\{\left(\sqrt{a_{n}} \vee 2^{-N}\right)^{2 \gamma\left(\tilde{\gamma}_{m}-1\right)}+(t-s)^{\gamma\left(\tilde{\gamma}_{m}-1\right)}+a_{n}^{2 \beta \gamma}\right\} d s
\end{aligned}
$$

Elementary calculations give

$$
\int_{t-\delta}^{t \wedge\left(t^{\prime}-\delta\right)}(t-s)^{-1 / 2} d s \leq 2\left(\sqrt{t^{\prime}-t} \wedge \sqrt{\delta}\right)
$$

and for $p \geq 0$,

$$
\int_{t-\delta}^{t \wedge\left(t^{\prime}-\delta\right)}(t-s)^{p} d s \leq \delta^{p}\left(\left(t^{\prime}-t\right) \wedge \delta\right) .
$$

For the integral in (7.8) consider separately the cases (i) $\sqrt{t-s}<2^{-N}$, (ii) $\sqrt{t-s} \geq \sqrt{a_{n}} \vee 2^{-N}$, and (iii) $2^{-N} \leq \sqrt{t-s}<\sqrt{a_{n}} \vee 2^{-N}$, the latter implying $\sqrt{a_{n}} \vee 2^{-N}=\sqrt{a_{n}}$, to bound $\hat{Q}_{T, 1, \delta}\left(t, t^{\prime}, x\right)$ by

$$
\begin{aligned}
& C_{5.2}(\omega) c_{4}(K)\left\{\int_{(t-\delta)^{+}}^{t \wedge\left(t^{\prime}-\delta\right)}(t-s)^{-1 / 2} d s 2^{-2 N \gamma \xi}\left[\left(\sqrt{a_{n}} \vee 2^{-N}\right)^{2 \gamma\left(\tilde{\gamma}_{m}-1\right)}+a_{n}^{2 \beta \gamma}\right]\right. \\
& +\int_{(t-\delta)^{+}}^{t \wedge\left(t^{\prime}-\delta\right)}(t-s)^{\gamma\left(\tilde{\gamma}_{m}+\xi-1\right)-\frac{1}{2}}+a_{n}^{2 \beta \gamma}(t-s)^{\gamma \xi-\frac{1}{2}} d s \\
& \left.+\int_{(t-\delta)^{+}}^{t \wedge\left(t^{\prime}-\delta\right)}(t-s)^{\gamma \xi-\frac{1}{2}} d s\left[a_{n}^{\gamma\left(\tilde{\gamma}_{m}-1\right)}+a_{n}^{2 \beta \gamma}\right]\right\} \\
& \leq 95.2(\omega) c_{5}(K)\left\{\left(\sqrt{\delta} \wedge \sqrt{t^{\prime}-t}\right) \bar{d}_{N}^{-2 \gamma \xi}\left[\vec{d}_{n, N}^{2 \gamma\left(\tilde{\gamma}_{m}-1\right)}+a_{n}^{2 \beta \gamma}\right]\right. \\
& +\left(\delta \wedge\left(t^{\prime}-t\right)\right)\left[\delta^{\gamma\left(\tilde{\gamma}_{m}+\xi-1\right)-\frac{1}{2}}+a_{n}^{2 \beta \gamma} \delta^{\gamma \xi-\frac{1}{2}}\right] \\
& \left.+\left(\delta \wedge\left(t^{\prime}-t\right)\right)\left[\delta^{\gamma \xi-\frac{1}{2}} a_{n}^{\gamma\left(\tilde{\gamma}_{m}-1\right)}+\delta^{\gamma \xi-\frac{1}{2}} a_{n}^{2 \beta \gamma}\right]\right\} .
\end{aligned}
$$

In the last we have used (7.9) and (17.10), and the fact that our choice of $\xi$ implies $\gamma \xi>1 / 2$ and hence our choices of $p$ are indeed non-negative when applying (7.10). Since $\delta \geq a_{n}$, the third term above is dominated by the second term. Therefore

$$
\begin{aligned}
& \hat{Q}_{T, 1, \delta}\left(t, t^{\prime}, x\right) \leq C_{5.2}(\omega) c_{6}(K)\{\left(\sqrt{\delta} \wedge \sqrt{t^{\prime}-t}\right)^{1-\frac{\eta_{1}}{2}} \bar{d}_{N}^{2 \gamma \xi+\frac{\eta_{1}}{2}}\left[\vec{d}_{n, N}^{2 \gamma\left(\tilde{\gamma}_{m}-1\right)}+a_{n}^{2 \beta \gamma}\right] \\
&\left.+\left(\delta \wedge\left(t^{\prime}-t\right)\right)^{1-\frac{\eta_{1}}{2}}\left[\delta^{\gamma\left(\tilde{\gamma}_{m}+\xi-1\right)-\frac{1}{2}+\frac{\eta_{1}}{2}}+a_{n}^{2 \beta \gamma} \delta^{\gamma \xi-\frac{1}{2}+\frac{\eta_{1}}{2}}\right]\right\} .
\end{aligned}
$$

Our choice of $\xi$ and conditions on $\eta_{1}$ imply that

$$
2 \gamma \xi+\frac{\eta_{1}}{2} \geq 2 \gamma \text { and } \gamma(\xi-1)+\frac{\eta_{1}}{2} \geq 0,
$$


and the required bound on $\hat{Q}_{T, 1, \delta}$ follows.

The above square function bounds suggest we will need a modified form of Lemma 5.7 to obtain

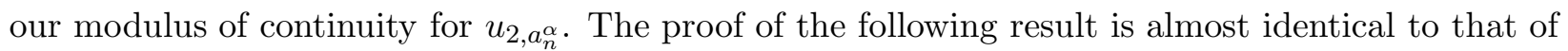
Lemma 5.7 and so is omitted.

Lemma 7.4 Let $c_{0}, c_{1}, c_{2}, k_{0}$ be positive (universal constants), $\eta \in(0,1 / 2)$, and $\Delta_{i}: \mathbb{N} \times(0,1] \rightarrow$ $\mathbb{R}_{+}, i=1,2$ satisfy $\Delta_{i}\left(n, 2^{-N+1}\right) \leq k_{0} \Delta_{i}\left(n, 2^{-N}\right)$ for all $n, N \in \mathbb{N}$ and $i=1,2$. For $n \in \mathbb{N}$ and $\tau$ in a set $S$ assume $\left\{Y_{\tau, n}(t, x):(t, x) \in \mathbb{R}_{+} \times \mathbb{R}\right\}$ is a real-valued continuous process. Assume for each $(n, \tau) \in \mathbb{N} \times S, K \in \mathbb{N}$, and $\beta \in[0,1 / 2]$, there is an $N_{0}(\omega)=N_{0}(n, \eta, K, \tau, \beta)(\omega) \in \mathbb{N}$ a.s., stochastically bounded uniformly in $(n, \tau, \beta)$, such that for any $N \in \mathbb{N},(t, x) \in \mathbb{R}_{+} \times \mathbb{R}$, if $d=d\left((t, x),\left(t^{\prime}, x^{\prime}\right)\right) \leq 2^{-N}$, then

$$
\begin{aligned}
& P\left(\left|Y_{\tau, n}(t, x)-Y_{\tau, n}\left(t^{\prime}, x^{\prime}\right)\right|>d^{\frac{1}{2}(1-\eta)} \Delta_{1}\left(n, 2^{-N}\right)+d^{1-\eta} \Delta_{2}\left(n, 2^{-N}\right),\right. \\
& \left.\quad(t, x) \in Z(N, n, K, \beta), N \geq N_{0}, t^{\prime} \leq T_{K}\right) \\
& \leq c_{0} \exp \left(-c_{1} d^{-\eta c_{2}}\right) .
\end{aligned}
$$

Then there is an $N_{0}^{\prime}(\omega)=N_{0}^{\prime}(n, \eta, K, \tau, \beta)(\omega) \in \mathbb{N}$ a.s., also stochastically bounded uniformly in $(n, \tau, \beta)$, such that for all $N \geq N_{0}^{\prime}(\omega),(t, x) \in Z(N, n, K, \beta)(\omega), d=d\left((t, x),\left(t^{\prime}, x^{\prime}\right)\right) \leq 2^{-N}$, and $t^{\prime} \leq T_{K}$,

$$
\left|Y_{\tau, n}(t, x)-Y_{\tau, n}\left(t^{\prime}, x^{\prime}\right)\right| \leq 2^{7} k_{0}^{2}\left[d^{\frac{1}{2}(1-\eta)} \Delta_{1}\left(n, 2^{-N}\right)+d^{1-\eta} \Delta_{2}\left(n, 2^{-N}\right)\right] .
$$

Proof of Proposition 5.14. The proof follows closely that of Proposition [5.8, using Lemma 7.4 in place of Lemma 5.7. Let $R=\frac{25}{\eta_{1}}$ and choose $\eta_{0} \in\left(\frac{1}{R}, \frac{\eta_{1}}{24}\right)$. Define $\bar{d}_{N}=d \vee 2^{-N}$, as usual, and set

$$
\hat{Q}_{a_{n}^{\alpha}}\left(t, x, t^{\prime}, x^{\prime}\right)=\sum_{i=1}^{2} \hat{Q}_{S, i, a_{n}^{\alpha}, \eta_{0}}\left(t, x, t^{\prime}, x^{\prime}\right)+\hat{Q}_{T, 1, a_{n}^{\alpha}}\left(t, t^{\prime}, x\right)+\hat{Q}_{T, 2, a_{n}^{\alpha}}\left(t, t^{\prime}, x^{\prime}\right) .
$$

By Lemmas 7.1, 7.2 and 7.3, for all $K \in \mathbb{N}$ (the restriction $K \geq K_{1}$ is illusory as these results only strengthen as $K$ increases) there is a constant $c_{1}\left(K, \eta_{1}\right)$ and $N_{2}\left(m, n, \eta_{1}, \varepsilon_{0}, K, \beta\right) \in \mathbb{N}$ a.s., stochastically bounded uniformly in $(n, \beta)$, such that for all $N \in \mathbb{N},(t, x) \in \mathbb{R}_{+} \times \mathbb{R}$,

$$
\text { on }\left\{\omega:(t, x) \in Z(N, n, K+1, \beta), N \geq N_{2}\right\} \text {, }
$$

$$
\begin{aligned}
& R_{0}^{\gamma} \hat{Q}_{a_{n}^{\alpha}}\left(t, x, t^{\prime}, x^{\prime}\right)^{1 / 2} \\
& \leq c_{1}\left(K, \eta_{1}\right)\left[a_{n}^{-\varepsilon_{0}}+2^{2 N_{2}}\right]\left\{\left(d \wedge a_{n}^{\frac{\alpha}{2}}\right)^{\frac{1}{2}\left(1-\frac{\eta_{1}}{2}\right)} \bar{d}_{N}^{\gamma}\left[\left(\bar{d}_{N} \vee a_{n}^{1 / 2}\right)^{\gamma\left(\tilde{\gamma}_{m}-1\right)}+a_{n}^{\beta \gamma}\right]\right. \\
& \left.\quad+\left(d \wedge a_{n}^{\frac{\alpha}{2}}\right)^{1-\frac{\eta_{1}}{2}}\left[a_{n}^{\frac{\alpha}{2}\left(\gamma \tilde{\gamma}_{m}-\frac{1}{2}\right)}+a_{n}^{\beta \gamma} a_{n}^{\frac{\alpha}{2}\left(\gamma-\frac{1}{2}\right)}\right]\right\}
\end{aligned}
$$

$$
\text { for all } t \leq t^{\prime} \leq T_{K},\left|x^{\prime}\right| \leq K+2 \text {. }
$$

Let $N_{3}=\frac{25}{\eta_{1}}\left[N_{2}+N_{4}\left(K, \eta_{1}\right)\right]$, where $N_{4}\left(K, \eta_{1}\right)$ is chosen large enough so that

$$
\begin{aligned}
c_{1}\left(K, \eta_{1}\right)\left[a_{n}^{-\varepsilon_{0}}+2^{2 N_{2}}\right] 2^{-N_{3} \eta_{1} / 8} & \leq c_{1}\left(K, \eta_{1}\right)\left[a_{n}^{-\varepsilon_{0}}+2^{2 N_{2}}\right] 2^{-6 N_{2}} 2^{-6 N_{4}\left(K, \eta_{1}\right)} \\
& \leq a_{n}^{-\varepsilon_{0}} 2^{-104} .
\end{aligned}
$$


Let $\Delta_{i, u_{2}}=2^{-100} \bar{\Delta}_{i, u_{2}}, i=1,2$. Assume $d \leq 2^{-N}$. Use (7.13) in (7.12) to see that for all $(t, x), N$, on

$$
\begin{aligned}
&\left\{\omega:(t, x) \in Z(N, n, K+1, \beta), N \geq N_{3}\right\}\left(\text { which implies }\left|x^{\prime}\right| \leq K+2\right), \\
& R_{0}^{\gamma} \hat{Q}_{a_{n}^{\alpha}}\left(t, x, t^{\prime}, x^{\prime}\right)^{1 / 2} \leq\left(d \wedge a_{n}^{\frac{\alpha}{2}}\right)^{\frac{1}{2}\left(1-\frac{3 \eta_{1}}{4}\right)} \Delta_{1, u_{2}}\left(m, n, 2^{-N}\right) / 16 \\
&+\left(d \wedge a_{n}^{\frac{\alpha}{2}}\right)^{1-\frac{5 \eta_{1}}{8}} \Delta_{2, u_{2}}(m, n) / 16 \quad \text { for all } t \leq t^{\prime} \leq T_{K}, x^{\prime} \in \mathbb{R} .
\end{aligned}
$$

Combine this with (7.1), (3.2), the definition of $\hat{Q}_{a_{n}^{\alpha}}$ and the Dubins-Schwarz theorem to conclude that for $t \leq t^{\prime}, x^{\prime} \in \mathbb{R}$, and $d\left((t, x),\left(t^{\prime}, x^{\prime}\right)\right) \leq 2^{-N}$,

$$
\begin{aligned}
& P\left(\left|u_{2, a_{n}^{\alpha}}(t, x)-u_{2, a_{n}^{\alpha}}\left(t^{\prime}, x^{\prime}\right)\right| \geq d^{\frac{1}{2}\left(1-\eta_{1}\right)} \Delta_{1, u_{2}}\left(m, n, 2^{-N}\right) / 4+d^{1-\eta_{1}} \Delta_{2, u_{2}}(m, n) / 4,\right. \\
& \left.(t, x) \in Z(N, n, K+1, \beta), N \geq N_{3}, t^{\prime} \leq T_{K}\right) \\
& \leq 3 P\left(\sup \left\{|B(u)|: u \leq\left[d^{\frac{1}{2}\left(1-\frac{3 \eta_{1}}{4}\right)} \Delta_{1, u_{2}}\left(m, n, 2^{-N}\right) / 16+d^{1-\frac{5 \eta_{1}}{8}} \Delta_{2, u_{2}}(m, n) / 16\right]^{2}\right\}\right. \\
& \left.\geq\left(d^{\frac{1}{2}\left(1-\eta_{1}\right)} \Delta_{1, u_{2}}\left(m, n, 2^{-N}\right)+d^{1-\eta_{1}} \Delta_{2, u_{2}}(m, n)\right) / 12\right) \\
& \leq 3 P\left(\sup _{u \leq 1}|B(u)| \geq d^{-\frac{\eta_{1}}{8}}\right) \leq c_{0} \exp \left(-\frac{d^{-\frac{\eta_{1}}{4}}}{2}\right) .
\end{aligned}
$$

Here $B(u)$ is a one-dimensional Brownian motion.

If $(t, x) \in Z(N, n, K, \beta), d \leq 2^{-N}$, and $t^{\prime} \leq t$, then as in the proof of Proposition 5.8. $\left(t^{\prime}, x^{\prime}\right) \in$ $Z(N-1, n, K+1, \beta)$ and one can interchange the roles of $(t, x)$ and $\left(t^{\prime}, x^{\prime}\right)$ and replace $N$ with $N-1$ in the above to conclude (as in (5.40) ),

$$
\begin{aligned}
& P\left(\left|u_{2, a_{n}^{\alpha}}(t, x)-u_{2, a_{n}^{\alpha}}\left(t^{\prime}, x^{\prime}\right)\right| \geq d^{\frac{1}{2}\left(1-\eta_{1}\right)} \Delta_{1, u_{2}}\left(m, n, 2^{-N}\right)+d^{1-\eta_{1}} \Delta_{2, u_{2}}(m, n),\right. \\
& \left.\quad(t, x) \in Z(N, n, K, \beta), N \geq N_{3}+1\right) \\
& \quad \leq c_{0} \exp \left(-\frac{d^{-\frac{\eta_{1}}{4}}}{2}\right) .
\end{aligned}
$$

(7.14) and (7.15) allow us to apply Lemma 7.4 with $\tau=\alpha, Y_{\tau, n}=u_{2, a_{n}^{\alpha}}$, and $k_{0}=4$. The result is then immediate once one recalls that $2^{7} k_{0}^{2} \Delta_{i, u_{2}}=2^{-89} \bar{\Delta}_{i, u_{2}}$.

\section{Incorporating Drifts}

Beginning in Section 3 we assumed that the drift $b$ is zero. Here we point out what additional reasoning is needed to include a drift $b$ satisfying (1.4). If $B(s, y)=b\left(s, y, X^{1}(s, y)\right)-b\left(s, y, X^{2}(s, y)\right)$, then (3.1) becomes

$$
\begin{aligned}
u(t, x) & =\int_{0}^{t} \int p_{t-s}(y-x) D(s, y) W(d s, d y)+\int_{0}^{t} \int p_{t-s}(y-x) B(s, y) d y d s \text { a.s. for all }(t, x) \\
& \equiv u_{D}(t, x)+u_{B}(t, x),
\end{aligned}
$$


and by (1.4),

$$
|B(s, y)| \leq B|u(s, y)| .
$$

If $u_{1, \delta}, u_{2, \delta}$ and $G_{\delta}$ are defined as at the beginning of Section 3, then as for (3.4), (3.5) and Lemma 3.1, but now using the ordinary Fubini theorem for $u_{B}$, we get

$$
\begin{aligned}
u_{1, \delta}(t, x) & =\int_{0}^{(t-\delta)^{+}} \int p_{t-s}(y-x) D(s, y) W(d s, d y)+\int_{0}^{(t-\delta)^{+}} \int p_{t-s}(y-x) B(s, y) d y d s \\
& \equiv u_{1, D, \delta}(t, x)+u_{1, B, \delta}(t, x), \\
\left.u_{2, \delta}(t, x)\right) & =\int_{(t-\delta)^{+}}^{t} \int p_{t-s}(y-x) D(s, y) W(d s, d y)+\int_{(t-\delta)^{+}}^{t} \int p_{t-s}(y-x) B(s, y) d y d s \\
& \equiv u_{2, D, \delta}(t, x)+u_{2, B, \delta}(t, x),
\end{aligned}
$$

and

$$
\begin{aligned}
-G_{\delta}^{\prime}(s, t, x) & \equiv F_{\delta}(s, t, x) \\
& =\int_{0}^{(s-\delta)^{+}} \int p_{(t \vee s)-r}^{\prime}(y-x) D(r, y) W(d r, d y)+\int_{0}^{(s-\delta)^{+}} \int p_{(t \vee s)-r}^{\prime}(y-x) B(r, y) d y d r \\
& \equiv F_{D, \delta}(s, t, x)+F_{B, \delta}(s . t, x) .
\end{aligned}
$$

In addition, no changes are required in the verification of $\left(P_{0}\right)$ (including the refinement noted in Remark 5.3) or the proof of Lemma 5.2.

The theorems in Section 5 apply directly to quantities like $u_{i, D, \delta}$ and $F_{D, \delta}$. The corresponding expressions $u_{i, B, \delta}$ and $F_{B, \delta}$ are in fact much easier to handle because we are dealing with a deterministic integral and so regularity properties are easy to read off directly from the bounds in Lemma 5.2, Furthermore, the Lipschitz condition on $b$ effectively sets $\gamma=1$ for these calculations. To illustrate this, we now prove a simple result which includes both Propositions 5.8 and 5.11 for $F_{B, a_{n}^{\alpha}}$ and only requires $\left(P_{0}\right)$, a consequence of the "crude" modulus Theorem 2.3, already noted above.

Proposition 8.1 For any $\eta_{1} \in\left(0, \frac{1}{2}\right)$ and $K \in \mathbb{N} \geq K_{1}$ there is an $N\left[\overline{8.1}\left(\eta_{1}, K\right)(\omega) \in \mathbb{N}\right.$ a.s. such that for all $n \in \mathbb{N}, \alpha \in[0,1]$ and $\beta \in\left[0, \frac{1}{2}\right], N \geq N \overline{8.1}\left(\eta_{1}, K\right),(t, x) \in Z(N, n, K, \beta)$, and $t^{\prime} \leq T_{K}$,

$$
\begin{gathered}
d=d\left((t, x),\left(t^{\prime}, x^{\prime}\right)\right) \leq 2^{-N} \text { and }\left|s^{\prime}-s\right| \leq N^{-1} \text { imply } \\
\begin{aligned}
\left|F_{B, a_{n}^{\alpha}}(s, t, x)-F_{B, a_{n}^{\alpha}}\left(s^{\prime}, t^{\prime}, x^{\prime}\right)\right| \leq 2^{-88} & {\left[\left|s^{\prime}-s\right|^{\frac{1}{2}} 2^{-N\left(1-\eta_{1}\right)}+\left|s^{\prime}-s\right|^{1-\frac{\eta_{1}}{2}}\right.} \\
& \left.+d^{1-\eta_{1}}\left(1+a_{n}^{-3 \alpha / 4} 2^{-2 N}\right)\right] .
\end{aligned}
\end{gathered}
$$

Proof. Let $\xi=1-\frac{\eta_{1}}{4}$ and assume first that

$$
N \geq N_{1}(0, \xi, K+1)+1,
$$

where $N_{1}$ is as in $\left(P_{0}\right)$ and Remark 5.3. Assume

$$
(t, x) \in Z(N, n, K, \beta), t^{\prime} \leq T_{K}, d \leq 2^{-N} \text { and }\left|s^{\prime}-s\right| \leq N^{-1} .
$$


One easily checks that $\left|t \wedge s-t^{\prime} \wedge s^{\prime}\right| \leq N^{-1}$ and so, by replacing $\left(s, s^{\prime}\right)$ with $\left(t \wedge s, t^{\prime} \wedge s^{\prime}\right)$, we may assume that $s \leq t$ and $s^{\prime} \leq t^{\prime}$. Define $\bar{s}=s \vee s^{\prime}$ and $\underline{s}=s \wedge s^{\prime}$. As before, $\left(t^{\prime}, x^{\prime}\right) \in$ $Z(N-1, n, K+1, \beta)$, and again, by interchanging $(t, x)$ with $\left(t^{\prime}, x^{\prime}\right)$, we may assume $t \leq t^{\prime}$ (this is the reason for the $K+1$ and adding 1 to $N_{1}$ in (8.3) ). As for (5.6), (8.2) implies

$$
\begin{aligned}
& \left|F_{B, a_{n}^{\alpha}}(s, t, x)-F_{B, a_{n}^{\alpha}}\left(s^{\prime}, t^{\prime}, x^{\prime}\right)\right| \\
& \leq \int_{\left(\underline{s}-a_{n}^{\alpha}\right)^{+}}^{\left(\bar{s}-a_{n}^{\alpha}\right)^{+}} \int\left|p_{t^{\prime}-r}^{\prime}\left(y-x^{\prime}\right)\right| B|u(r, y)| d y d r+\int_{0}^{\left(s-a_{n}^{\alpha}\right)^{+}} \int\left|p_{t^{\prime}-r}^{\prime}\left(y-x^{\prime}\right)-p_{t-r}^{\prime}(y-x)\right| B|u(r, y)| d y d r \\
& \equiv T_{1}+T_{2} .
\end{aligned}
$$

To bound $T_{1}$, we may assume $\bar{s} \geq a_{n}^{\alpha}$. Elementary inequalities using $t^{\prime} \leq T_{K} \leq K$, show that for $p \geq 0$,

$$
\begin{aligned}
\int\left|p_{t^{\prime}-r}^{\prime}\left(y-x^{\prime}\right)\right|\left|y-x^{\prime}\right|{ }^{p} e^{\left|y-x^{\prime}\right|} d y & \leq\left(t^{\prime}-r\right)^{-1} \int\left|y-x^{\prime}\right|^{p+1} e^{\left|y-x^{\prime}\right|} p_{t^{\prime}-r}\left(y-x^{\prime}\right) d y \\
& \leq c_{1}(K, p)\left(t^{\prime}-r\right)^{\frac{p-1}{2}} .
\end{aligned}
$$

Now apply Lemma 5.2 with $m=0$ to see that

$$
\begin{aligned}
& T_{1} \leq B \sqrt{9[\overline{5.2}} \int_{\left(\underline{s}-a_{n}^{\alpha}\right)^{+}}^{\bar{s}-a_{n}^{\alpha}} \int\left|p_{t^{\prime}-r}^{\prime}\left(y-x^{\prime}\right)\right| e^{\left|y-x^{\prime}\right|} e^{\left|x-x^{\prime}\right|}\left((\sqrt{t-r}+|y-x|) \vee 2^{-N}\right)^{\xi} d y d r \\
& \leq c_{2}(K) \sqrt{\overline{C_{5.2}}} \int_{\left(\underline{s}-a_{n}^{\alpha}\right)+}^{\bar{s}-a_{n}^{\alpha}} \int\left|p_{t^{\prime}-r}^{\prime}\left(y-x^{\prime}\right)\right| e^{\left|y-x^{\prime}\right|}\left[\left(\left|x-x^{\prime}\right| \vee 2^{-N}\right)^{\xi}+\sqrt{t^{\prime}-r^{\xi}}+\left|y-x^{\prime}\right|^{\xi}\right] d y d r \\
& \leq c_{3}(K) \sqrt{q[\overline{5.2}} \int_{\left(\underline{s}-a_{n}^{\alpha}\right)^{+}}^{\bar{s}-a_{n}^{\alpha}}\left(t^{\prime}-r\right)^{-1 / 2}\left[2^{-N \xi}+\left(t^{\prime}-r\right)^{\xi / 2}\right] d r \quad\left(\text { by }(\underline{8.51}) \text { and }\left|x-x^{\prime}\right| \leq 2^{-N}\right) \\
& \leq c_{4}(K) 2^{2 N_{1}(\omega)}\left[\left|s^{\prime}-s\right|^{1 / 2} 2^{-N\left(1-\frac{\eta_{1}}{4}\right)}+\left|s^{\prime}-s\right|^{1-\frac{\eta_{1}}{8}}\right] \\
& \leq c_{4}(K) 2^{2 N_{1}(\omega)}\left[2^{-N \eta_{1} / 2}+\left|s^{\prime}-s\right|^{\eta_{1} / 4}\right]\left[\left|s^{\prime}-s\right|^{1 / 2} 2^{-N\left(1-\eta_{1}\right)}+\left|s-s^{\prime}\right|^{1-\frac{\eta_{1}}{2}}\right] \\
& \leq c_{4}(K) 2^{2 N_{1}(\omega)}\left[2^{-N \eta_{1} / 2}+N^{-\eta_{1} / 4}\right]\left[\left|s^{\prime}-s\right|^{1 / 2} 2^{-N\left(1-\eta_{1}\right)}+\left|s-s^{\prime}\right|^{1-\frac{\eta_{1}}{2}}\right] .
\end{aligned}
$$

Recall in the above that we may set $\varepsilon_{0}=0$ in the definition of $C_{5.2}$ (see Remark [5.3).

For $T_{2}$ we use both $|u(r, y)| \leq K e^{|y|}$ for $r \leq t^{\prime} \leq T_{K}\left(K \geq K_{1}\right)$ and Lemma 5.2 with $m=0$ to 
write (we may assume $s>a_{n}^{\alpha}$ ),

$$
\begin{aligned}
& T_{2} \leq \int_{0}^{s-a_{n}^{\alpha}} \int\left|p_{t-r}^{\prime}\left(y-x^{\prime}\right)-p_{t-r}^{\prime}(y-x)\right| B K e^{|y|} 1\left(|y-x|>\left(t^{\prime}-r\right)^{\frac{1}{2}-\frac{\eta_{1}}{4}} \vee\left(2\left|x^{\prime}-x\right|\right)\right) d y d r \\
& +\left.\int_{0}^{s-a_{n}^{\alpha}} \int\left|p_{t-r}^{\prime}\left(y-x^{\prime}\right)-p_{t-r}^{\prime}(y-x)\right| B \sqrt{G_{5.2}}\right|^{|y-x|}\left[(\sqrt{t-r}+|y-x|) \vee 2^{-N}\right]^{\xi} \\
& \times 1\left(|y-x| \leq\left(t^{\prime}-r\right)^{\frac{1}{2}-\frac{\eta_{1}}{4}} \vee\left(2\left|x^{\prime}-x\right|\right)\right) d y d r \\
& \leq c_{5}(K) \sqrt{G 5.2}\left[\int_{0}^{s-a_{n}^{\alpha}} \int\left|p_{t-r}^{\prime}\left(y-x^{\prime}\right)-p_{t-r}^{\prime}(y-x)\right| e^{2|y-x|}\right. \\
& \times 1\left(|y-x|>\left(t^{\prime}-r\right)^{\frac{1}{2}-\frac{\eta_{1}}{4}} \vee\left(2\left|x^{\prime}-x\right|\right)\right) e^{-|y-x|} d y d r \\
& \left.+\int_{0}^{s-a_{n}^{\alpha}} \int\left|p_{t-r}^{\prime}\left(y-x^{\prime}\right)-p_{t-r}^{\prime}(y-x)\right| 1(|y-x| \leq 2(2 K+1)) d y\left(\left(t^{\prime}-r\right)^{\frac{1}{2}-\frac{\eta_{1}}{4}} \vee\left|x^{\prime}-x\right| \vee 2^{-N}\right)^{\xi} d r\right] \\
& \leq c_{6}(K) \sqrt{9[5.2}\left[\int_{0}^{s-a_{n}^{\alpha}} \int\left(p_{t-r}^{\prime}\left(y-x^{\prime}\right)-p_{t-r}^{\prime}(y-x)\right)^{2} e^{4|y-x|}\right. \\
& \times 1\left(|y-x|>(t-r)^{\frac{1}{2}-\frac{\eta_{1}}{4}} \vee\left(2\left|x^{\prime}-x\right|\right)\right) d y^{1 / 2}\left(\int e^{-2|y-x|} d y\right)^{1 / 2} d r \\
& \left.+\int_{0}^{s-a_{n}^{\alpha}} \int\left(p_{t-r}^{\prime}\left(y-x^{\prime}\right)-p_{t-r}^{\prime}(y-x)\right)^{2} d y^{1 / 2}(2(2 K+1))^{1 / 2}\left[(t-r)^{1 / 2} \vee 2^{-N}\right)^{\xi\left(1-\frac{\eta_{1}}{2}\right)} d r\right],
\end{aligned}
$$

where in the last line for the second term we use $d \leq 2^{-N}$ and

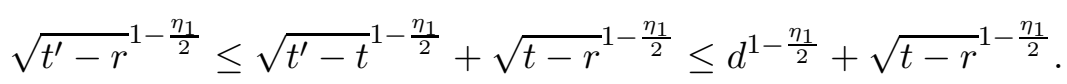


Now apply Lemma 4.4 and conclude

$$
\begin{aligned}
& T_{2} \leq c_{7}\left(K, \eta_{1}\right) \sqrt{C_{5.2}}\left\{\int_{0}^{s-a_{n}^{\alpha}}(t-r)^{-3 / 4} \exp \left\{\frac{-\eta_{1}(t-r)^{-\eta_{1} / 2}}{128}\right\}\left(1 \wedge \frac{d^{2}}{t-r}\right)^{\frac{1}{2}-\frac{\eta_{1}}{4}} d r\right. \\
& \left.+\int_{0}^{s-a_{n}^{\alpha}}(t-r)^{-3 / 4}\left(1 \wedge \frac{d^{2}}{t-r}\right)^{1 / 2}\left[(t-r)^{1 / 2} \vee 2^{-N}\right]^{\xi\left(1-\frac{\eta_{1}}{2}\right)} d r\right\} \\
& \leq c_{7}\left(K, \eta_{1}\right) \sqrt{9 \overline{5.2}}\left\{\int_{0}^{s-a_{n}^{\alpha}}(t-r)^{-3 / 4} \exp \left\{\frac{-\eta_{1}(t-r)^{-\eta_{1} / 2}}{128}\right\}\left(1 \wedge \frac{d^{2}}{t-r}\right)^{\frac{1}{2}-\frac{\eta_{1}}{4}} d r\right. \\
& +\int_{0}^{s-a_{n}^{\alpha}}(t-r)^{-\frac{3}{4}+\frac{\xi}{2}\left(1-\frac{\eta_{1}}{2}\right)}\left(1 \wedge \frac{d^{2}}{t-r}\right)^{1 / 2} d r \\
& \left.+\int_{0}^{s-a_{n}^{\alpha}} 1\left(r \geq t-2^{-2 N}\right)(t-r)^{-\frac{3}{4}}\left(1 \wedge \frac{d^{2}}{t-r}\right)^{1 / 2} d r 2^{-N \xi\left(1-\frac{\eta_{1}}{2}\right)}\right\} \\
& \leq c_{8}\left(K, \eta_{1}\right) \sqrt{95.2}\left\{\int_{0}^{s-a_{n}^{\alpha}}\left(1 \wedge \frac{d^{2}}{t-r}\right)^{\frac{1}{2}-\frac{\eta_{1}}{4}} d r+d\right. \\
& \left.+1\left(a_{n}^{\alpha}<2^{-2 N}\right) d\left(d^{2} \vee a_{n}^{\alpha}\right)^{-1 / 4} 2^{-N \xi\left(1-\frac{\eta_{1}}{2}\right)}\right\} \\
& \leq c_{9}\left(K, \eta_{1}\right) \sqrt{q 5.2}\left\{d^{1-\frac{\eta_{1}}{2}}+1\left(a_{n}^{\alpha}<2^{-2 N}\right) d a_{n}^{-3 \alpha / 4} a_{n}^{\alpha / 2} 2^{-N \xi\left(1-\frac{\eta_{1}}{2}\right)}\right\} \\
& \leq c_{9}\left(K, \eta_{1}\right) \sqrt{95.2} d^{1-\frac{3 \eta_{1}}{4}}\left[1+a_{n}^{\frac{-3 \alpha}{4}} 2^{-\frac{3 N \eta_{1}}{4}} 2^{-N} 2^{-N\left(1-\frac{3 \eta_{1}}{4}\right)}\right] \\
& \leq c_{10}\left(K, \eta_{1}\right) 2^{2 N_{1}(\omega)} 2^{-N \eta_{1} / 4} d^{1-\eta_{1}}\left[1+a_{n}^{-\frac{3 \alpha}{4}} 2^{-2 N}\right] .
\end{aligned}
$$

We have used Lemma 4.1 in the above with a bit of algebra to see which case applies, and in the last two lines again used $d \leq 2^{-N}$. (8.6) and (8.7) together show there is an $N_{\overline{8.1}}\left(\eta_{1}, K\right)(\omega) \in \mathbb{N}$ a.s. such that $N_{8.1}\left(\eta_{1}, K\right) \geq N_{1}(0, \xi, K+1)+1$ and, if $N \geq N_{8.1}$, then

$$
T_{1}+T_{2} \leq 2^{-88}\left[\left|s^{\prime}-s\right|^{1 / 2} 2^{-N\left(1-\eta_{1}\right)}+\left|s^{\prime}-s\right|^{1-\frac{\eta_{1}}{2}}+d^{1-\eta_{1}}\left[1+a_{n}^{-\frac{3 \alpha}{4}} 2^{-2 N}\right]\right] .
$$

A bit of arithmetic shows that the above bound in the contexts of Propositions 5.8 and 5.11 lead to upper bounds that are bounded by the ones obtained there for increments of $F_{D, a_{n}^{\alpha}}$. For Proposition 5.8 one only needs the first two terms in $\bar{\Delta}_{u_{1}^{\prime}}$ and we leave this easy check for the reader. For Proposition 5.11 we may set $\left(s^{\prime}, t^{\prime}, x^{\prime}\right)=(t, t, x)$ in the above so that the upper bound becomes

$$
\begin{aligned}
& 2^{-88}\left[|t-s|^{1 / 2} 2^{-N\left(1-\eta_{1}\right)}+|t-s|^{1-\frac{\eta_{1}}{2}}\right] \\
& \leq 2^{-88}\left[2^{-N\left(1-\eta_{1}\right)}+(t-s)^{\frac{1-\eta_{1}}{2}}\left(\sqrt{t-s} \vee \sqrt{a_{n}}\right)^{\gamma \tilde{\gamma}_{m}-\frac{3}{2}}\right],
\end{aligned}
$$

which is bounded by two of the terms on the right-hand side of the upper bound in Proposition 5.11. Hence we may combine these bounds for $F_{B, a_{n}^{\alpha}}$ with those derived in Propositions 5.8 and 5.11 for $F_{D, a_{n}^{\alpha}}$ and hence complete the proofs of Propositions 5.8 and 5.11 (and hence also Corollary [5.9) for solutions with Lipschitz drifts.

We omit the analogues of the above for Propositions 5.12 and 5.14 as they are even simpler. The proof of Propositions 5.13 and 5.1 now proceed as before. With Propositions [5.1, 5.13, 5.14, and 
5.11, and Corollary 5.9 in hand, the proof of Proposition 3.3 may now be completed for Lipschitz drifts $b$, exactly as in Section 6. Then verification of the hypotheses of Proposition 2.1 may now be completed for Lipschitz drifts $b$ exactly as in Section 3 and this finishes the proof of Theorem 1.2,

\section{References}

[Dal99] R. C. Dalang. Extending martingale measure stochastic integrals with applications to spatially homogeneous SPDEs. Electronic Journal of Probability, 4:1-29, 1999.

[Kur07] T. G. Kurtz. The Yamada-Watanabe-Engelbert theorem for general stochastic equations and inequalities. Elect. J. Prob., 12:951-965, 2007.

[M66] P. A. Meyer Probability and Potentials Blaisdell, Waltham, 1966.

[MPS06] L. Mytnik, E. Perkins, A. Sturm. On pathwise uniqueness for stochastic heat equations with non-Lipschitz coefficients. Annals of Probability, 34: 1910-1959, 2006, arXiv:math/0507545.

[Myt99] L. Mytnik. Uniqueness for a competing species model. Canadian J. Math., 51:372-448, 1999.

[Per02] E. Perkins. Dawson-Watanabe Superprocesses and Measure-valued Diffusions, in Ecole d'Eté de Probabilités de Saint Flour 1999, Lect. Notes. in Math. 1781, Springer-Verlag, 2002.

[SS02] M. Sanz-Solé and M. Sarrà. Hoelder continuity for the stochastic heat equation with spatially correlated noise, in Progress in Probability, Stochastic Analysis, Random Fields and Applications, Birkhaeuser Basel, 2002.

[Shi94] T. Shiga Two contrasting properties of solutions for one-dimensional stochastic partial differential equations. Canadian J. Math., 46:415-437, 1994.

[SV79] D. W. Stroock and S. R. S. Varadhan. Multidimensional Diffusion Processes. SpringerVerlag, Berlin 1979.

[Wal86] J. Walsh. An Introduction to Stochastic Partial Differential Equations, in Ecole d'Eté de Probabilités de Saint Flour 1984, Lect. Notes. in Math. 1180, Springer, Berlin, 1986.

[YW71] T. Yamada and S. Watanabe. On the uniqueness of solutions of stochastic differential equations. J. Math. Kyoto U., 11, 155-167, 1971. 